\title{
Elemental analysis of ischemic and reperfused rat heart tissue using the proton microprobe
}

Citation for published version (APA):

Verhoef, B. A. W. (1997). Elemental analysis of ischemic and reperfused rat heart tissue using the proton microprobe. [Doctoral Thesis, Maastricht University]. Universiteit Maastricht. https://doi.org/10.26481/dis.19971218bv

Document status and date:

Published: 01/01/1997

DOI:

10.26481/dis.19971218bv

Document Version:

Publisher's PDF, also known as Version of record

\section{Please check the document version of this publication:}

- A submitted manuscript is the version of the article upon submission and before peer-review. There can be important differences between the submitted version and the official published version of record.

People interested in the research are advised to contact the author for the final version of the publication, or visit the DOI to the publisher's website.

- The final author version and the galley proof are versions of the publication after peer review.

- The final published version features the final layout of the paper including the volume, issue and page numbers.

Link to publication

\footnotetext{
General rights rights.

- You may freely distribute the URL identifying the publication in the public portal. please follow below link for the End User Agreement:

www.umlib.nl/taverne-license

Take down policy

If you believe that this document breaches copyright please contact us at:

repository@maastrichtuniversity.nl

providing details and we will investigate your claim.
}

Copyright and moral rights for the publications made accessible in the public portal are retained by the authors and/or other copyright owners and it is a condition of accessing publications that users recognise and abide by the legal requirements associated with these

- Users may download and print one copy of any publication from the public portal for the purpose of private study or research.

- You may not further distribute the material or use it for any profit-making activity or commercial gain

If the publication is distributed under the terms of Article $25 \mathrm{fa}$ of the Dutch Copyright Act, indicated by the "Taverne" license above, 
Elemental analysis of ischemic and reperfused rat heart tissue using the proton microprobe 
O Bas Verhoef Nuenen 1997

ISBN 90-9011189-1

Universiteitsdrukkerij, Technische Universiteit Eindhoven

Omslag: Ben Mobach, naar een idee van Peter Frederik 


\section{Elemental analysis of ischemic and reperfused rat heart tissue using the proton microprobe}

\section{PROEFSCHRIFT}

ter verkrijging van de graad van doctor aan de Universiteit Maastricht, op gezag van de Rector Magnificus, Prof. mr. M.J. Cohen, volgens het besluit van het College van Decanen in het openbaar te verdedigen op donderdag 18 december 1997 om 10.00 uur

door

\section{BASTIAAN ABRAM WILLEM VERHOEF}

geboren te Spijk op 23 december 1967 


\section{Promotores:}

Prof dr. GI. van der Vusse

Prof dr. M.I.A. de Voigt

Co-piromotores:

Dr. P.M. Frederik

Dr ir PHA Mutsaers

\section{Beoordelingscommissie:}

Prof dr. Th. Arts (voorzitter)

Prof dr. H. Bouma

Prof dr. M.P. van Dieijen-Visser

Prof. dr. ir. J.J.M de Goeji

Prof. dr. R.S. Reneman 
Aan Ageeth 



\section{Contents}

Chapter 1 General introduction 1

1.0 Introduction . . . . . . . . . . . . . . . . 1

1.1 Physiological background . . . . . . . . . . 1

1.2 Techniques to analyse elemental concentrations in cardiac tissue, with special reference to the proton microprobe . . . . . . . . 5

1.3 Aim of the study and outline of thesis . . . . . . . . . . . . 6

Chapter 2 Elemental analysis with X-rays; basic aspects 7

2.0 Introduction . . . . . . . . . . . . . . . 7

2.1 Particle Induced X-ray Emission . . . . . . . . . . . . . 9

2.1.1 General description of thick target PLXE . . . . . . . . 9

2.1.2 Theoretical evaluation and experimental verification of thick target PIXE for light elements in biological samples . . . 14

2.2 Determination of the local areal mass density . . . . . . . . . . $\quad 18$

2.2.1 Nuclear Back and Forward Scattering spectroscopy . . . . . 19

2.2.2 Scanning Transmission Ion Microscopy . . . . . . . . 22

2.3 Determination of elemental concentration and limit of detection . . . . . 23

2.4 Summary and concluding remarks . . . . . . . . . . . . 29

Chapter 3 Practical comparison between PIXE and EPMA 31

3.0 Introduction . . . . . . . . . . . . . . . . . 31

3.1 Preparation of calibration samples . . . . . . . . . . . . 31

3.2 Electron and proton probe $\mathrm{X}$-ray microanalysis . . . . . . . . 33

3.3 Results and discussion . . . . . . . . . . . . . . . 35

3.4 Summary and concluding remarks . . . . . . . . . . . 39

Chapter 4 Technical aspects of proton microprobe analysis of biological material

4.0 Introduction . . . . . . . . . . . . . . . 41 41

4.1 Experimental set-up . . . . . . . . . . . . . . . 41

4.2 Imaging techniques . . . . . . . . . . . . . . . 51

4.2.1 Light microscopy . . . . . . . . . . . . . . 51

4.2.2 Scanning Transmission Ion Microscopy (STIM) . . . . . . . 53

4.2 .3 Electron microscopy . . . . . . . . . . . . . . . 58

4.2 .4 Secondary electron imaging . . . . . . . . . . . 59

4.3 Data acquisition and data analysis . . . . . . . . . . . . . 60

4.4 Summary and concluding remarks . . . . . . . . . . . . 65 
Chapter 5 PIXE microanalysis of elements of normoxic, ischemic and reperfused rat hearts; preliminary resulls

5.0 Introduction

5.1 Materials and methods

5.1.1 Perfusion experiments and sample preparation . . . 68

5.1 .2 Proton and electron probe analysis . . . . . . . . . 73

5.2 Results and discussion : . . . . . . . . . . . 74

5.2 .1 Electron microscope results . . . . . . . . . 74

5.2.2 Preliminary proton microprobe results . . . . . . . . . . 78

5.3 Summary and concluding remarks

Chapter 6 Proton microprobe analysis of rat hearts subjected to calcium paradox experiments

6.0 Introduction

6.1 Materials and methods

6.2 Results

6.3 Discussion

6.4 Summary and concluding remarks

Chapter 7 Cellular and intracellular heterogeneity of elemental content in normoxic, ischemic and reperfused rat hearts

7.0 Introduction

7.1 Materials and methods

7.2 Results

7.3 Discussion

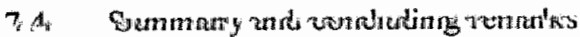

\section{Chapter 8 General discussion}

8.0 Introduction .

8.1 Summary and discussion of results

8.1.1 Comparison of proton and electron microprobe results . . . . . $\quad 128$

8.1.2 (Patho)-physiological significance of proton microprobe results . $\quad 129$

8.2 Future developments

8.2.1 Use of line scans

8.2.2 Improvement of detection efficiency

8.2.3 Improvement of spatial resolution

8.2.4 How to obtain statistically relevant results in future

8.2.5 Use of independent markers to distinguish intra-and extracellular

8.2.6 The possible role of heavy metals in ischemia and reperfusion

8.2.7 Application of the proton microprobe for clinical problems 
References .

Summary . . . . . . . . . . . . . . . . . 143

Samenvatting . . . . . . . . . . . . . . . 147

Publications and abstracts . . . . . . . . . . . . 151

Curriculum Vitae

Dankwoord . . . . . . . . . . . . . . 155 


\section{Chapter 1}

\section{General introduction}

\subsection{Introduction}

Most of the 40 worldwide available proton microprobe instruments are developed and used at institutes involved in nuclear and atomic physics instead of biology and medicine. However, in recent years interest from the biomedical field to use a proton microprobe for trace element analysis is growing [DOY97]. Due to the limited availability and the technical difficulty to operate this instrument few microanalysts involved in biomedical research have access to this powerful analytical tool. At present about 15 groups. [WAT95], among which the Eindhoven proton microprobe group, are involved in proton microprobe analysis of biological specimens for scientific and/or medical purposes. However, the use of animals and the preparation of biological material requires specialist skills and knowledge commonly not available at nuclear physics groups. For this reason an increasing number of proton microprobe groups is inwolved in collaborations with biomedical research groups.

The study described in this thesis was performed in the context of a cooperation between the Department of Physics at the Eindhoven University of Technology and the Department of Physiology of the Maastricht University. A short description of the plysiological background of this study is given in Section 1.1. The aim of the study and the motivation for the use of the proton microprobe instead of other available microanalytical techniques is described in Section 1.2. Finally, the outline of this thesis is discussed in Section 1.3.

\subsection{Physiological background}

When the heart is functioning properly, a continuous flow of blood is pumped through the vascular system of the body. The contractile elements of the heart are the cardiac muscle cells or myocardial fibres. The cells of the working myocardium contain large numbers of contractile proteins. These proteins, including actin, myosin and troponin, are organized in myofibrils. The cardiac muscle cell, with dimensions of 50-100 $\mu \mathrm{m}$ length and 10-20 $\mu \mathrm{m}$ thickness, is enclosed by a plasma membrane (the sarcolemma) composed of phospholipids, cholesterol and proteins. The sarcolemma forms a barrier between the fluid within the cell (the cytosol) and the extracellular environment. The intracellular compartment is occupied by organelles, such as the nucleus and the mitochondria. The myofibrils are surrounded by an intracellular membrane network called sarcoplasmic reticulum (SR). In addition small molecules, among them organic and inorganic ions, are dissolved in the cytosol. The ion concentration in the extracellular space is different from the cytosolic concentrations of each ion. Both cytosolic and extracellular concentrations are kept within physiologically acceptable limits, and the values of the concentration of a selection of ions are given in Table 1.1. It should be noted that values from literature vary considerably. For this reason Table 1.1 primarily gives a rough indication of the intra- and extracellular ion concentration of several elements. 
Table 1.1: Rough indications of the extracellular and intracellular concentration of a selection of ions in a muscle cell of a warm-blooded amimal Data are derived from [SCH89] and [BER88].

\begin{tabular}{ccc}
\hline Ion & $\begin{array}{c}\text { Extracellular concentration } \\
(\mathrm{mmol} / \mathrm{l})\end{array}$ & $\begin{array}{c}\text { Intracellular concentration } \\
(\mathrm{mmol} /)\end{array}$ \\
\hline $\mathrm{Na}^{+}$ & 145 & 12 \\
$\mathrm{~K}^{++}$ & 4 & 155 \\
$\mathrm{Mg}^{2+}$ & 0.6 & 20 \\
$\mathrm{Ca}^{2+}$ & 2 & $10^{-5}-10^{-4}$ \\
$\mathrm{Cl}^{+}$ & 120 & 4 \\
\hline
\end{tabular}

The intracellular $\mathrm{Na}^{+}$concentration is about 10 times lower than the concentration outside the cell, whereas for $\mathrm{K}^{*}$ the opposite applies. The largest ion gradient across the cell membrane is that of $\mathrm{Ca}^{2+}$, with a cytosolic concentration that is about 10,000 times lower than outside the cell. It should be noted that the values displayed in Table 1.1 are valid for ions in an aqueous environment. These ions can either be free in solution or complexed to proteins, such as albumin, etc. Furthermore, it should be emphasized that ions present in subcellular organelles, such as mitochondria and the SR, are not included in this table, so their local concentration can be considerably higher Large amounts of intracellular $\mathrm{Ca}^{2+}$, for example, are stored in the SR.

Ions, such as $\mathrm{Na}^{*}, \mathrm{~K}^{*}, \mathrm{Mg}^{2+*}$ and $\mathrm{Ca}^{2+}$, play an important role in several physiological processes, such as the excitation-contraction coupling. The sodium ion gradient is essential for the electrical activity of the heart cell and, indirectly, participates in the regulation of myocardial contractility. The calcium ion plays a key role in muscle contraction. This ion is released from intracellular storage regions, the transverse tubules system (T-system) and the SR, thereby initiating a contraction of the myofibrils. The muscle relaxes again when the calcium ions are pumped back into the SR and extracellular space. The presence of $\mathrm{Mg}^{2+}$ is required to release energy from the hydrolytic splitting of adenosine tri-phosphate (ATP) needed for contraction. Moreover, this ion is essential for the functioning of both the sarcolemmal Na-K punp and the sarcoplasmic reticulum calcium pump.

There is a constant exchange of ions between the intra- and extracellular compartment (see 1ig. 1.1). First the ions migrate by diffusion through selective ion channels embedded in the cell membrane. This process should eventually result in the elimination of the differences between intra- and extracellular ion concentrations. However, ions are charged particles and the flow of these particles creates an electrical field that counteracts the flow of ions due to the chemical concentration gradient. As a result for each ion there is an equilibrium potential at which the diffusion of the ions across the cell membrane is compensated by the action of the electrical field. The intracellular concentration of some ions, such as $\mathrm{Na}^{+}$and $\mathrm{K}^{+}$, is not stabilized by these two processes of passive diffusion. Additional active transport processes, i.e., membrane proteins that transport ions across the sarcolemma against the concentration and potential gradients, are responsible for the stability of the ion gradients. One of the most important active transport 
processes is the $\mathrm{Na}-\mathrm{K}$ pump, which removes $\mathrm{Na}^{+}$from the cell interior and moves $\mathrm{K}^{4}$ into it: In addition the sarcolemma also contains a calcium pump, which pumps $\mathrm{Ca}^{2+}$ out of the cell. To maintain the cytosolic $\mathrm{Ca}^{2+}$ concentration at the extremely low level the regulation of this calcium pump is calcium dependent, by which calcium entry stimulates the removal of $\mathrm{Ca}^{2+}$ ions from the intracellular compartment [SCH89].

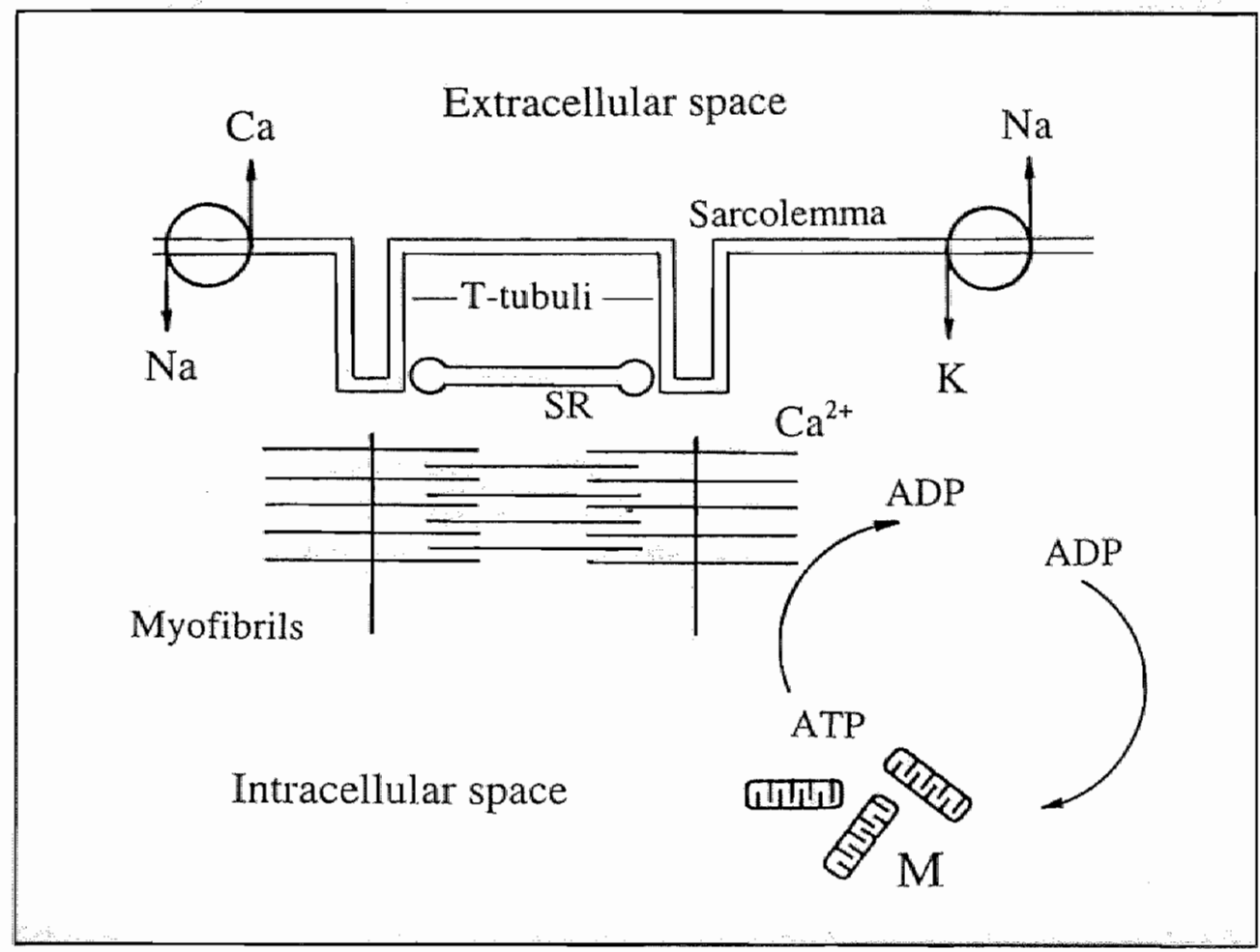

Figure 1.1 Schematic representation of the cell. The Na-Ca pump and the Na-K pump are located at the sarcolemma; $M$, mitochondria: SR, sarcoplasmic reticulum.

The energy consumption of both the Na-K pump and calcium pump is met by the energyproducing processes in the heart, from which the production of the energy-rich ATP, is the most important. The energy released during the hydrolytic splitting of ATP into adenosine diphosphate (ADP) and phosphate is used particularly for muscle contraction and for the active transport of ions across the membrane. The bulk of ATP is produced inside the mitochondria and requires an uninterrupted supply of substrates, particularly glucose and fatty acids, and of oxygen. When the cells are deprived from oxygen (e.g. during hypoxia or ischemia) ATP will be produced by means of anaerobic glycolysis of glycogen stored in the cells. However, anaerobic glycolysis, givinig rise to accumulation of $\mathrm{H}^{+}$(acidosis) and lactate, can satisfy only part of the myocardial energy 
demand and the amount of glycogen stored is limited. Moreover, the process of anaerobic ATP production is inhibited by acidosis and lactate accumulation in the cell at a time that still significant amounts of glycogen are present [OP191].

The above implies that the function of the tonic pumps is hampered in case the supply of substrates and oxygen is stopped. Consequently an interruption of coronary perfusion (ischemia) will create a considerable imbalance in the ion concentrations of the oxygen deprived cells, resulting in loss of function within a few minutes and finally cell death. Therefore, myocardial cells deprived from blood flow will inevitably die unless flow is timely restored (reperfusion). Paradoxically, reperfusion per se might add to the damage inflicted upon the heart during the ischemic episode [PIP90]. Under experimental conditions myocardial cell death begins after 15 to $40 \mathrm{~min}$ of total ischemia. In the heart in sitw, cell death starts in the inner wall of the myocardium (the endocarclium) and subsequently extends towards the outer myocardial wall (the epicardium).

To date in cardiology no unique answer to the mechanism of ischemic cell death has been given [PIP90]. It is very likely, however, that the fracturing of the sarcolemma is the proximate cause of irreversible cellular damage due to ischemia and reperfusion. Two possible explanations for the mechanisms underlying disnuption of the cellular membrane are (i) the degradation of the membrane phospholipid pool and (ii) the fracturing of the sarcolemma by physical forces, like osmotic load (cell swelling) and mechanical stress (e.g., hypercontraction, initiated by an excessive entry of calcium into the intracellular compartment). In the latter case, however, it was shown by Jennings and colleagues [JEN86] that the membrane has to be weakened prior to rupture due to enhanced physical stress. This destabitization of the sarcolemma might for instance be due to the impaired anchoring of the membrane to the cytoskeleton and/or accumulation of phospholipid degradation products in the sarcolemma [PIP90].

A hypothesis for the mechanism of ischemic cell death is that cell injury is caused by the inhibition of the ionic pumps, followed by alterations in the cellular concentrations of ions such as $\mathrm{Na}^{*}, \mathrm{Mg}^{2+}, \mathrm{K}^{*+}$ and $\mathrm{Ca}^{2+}$. It has been proposed by several authors [REI86, BUJ89] that the inhibition of the $\mathrm{Na}-\mathrm{K}$ pump results in a lower $\mathrm{Na}^{+}$gradient across the sarcolemma, which in turn causes the influx of $\mathrm{Ca}^{2+}$ into the intracellular space through activation of the $\mathrm{Na}$ - $\mathrm{Ca}$ exchanger. The resulting elevated intracellular calcium levels are believed to play a crucial role in the onset of events that lead to irreversible damage of the myocardial cells. Several studies suggest that raised extracellular magnesium is protective in myocardial ischemia [JYN95, TE093]. This antiischemic action may be due to a reduction of the massive influx of calcium and thus of the resulting damage. Loss of potassium from the myocardial cells leads to loss of proper electrical function of the heart [WEI82], whereas some studies of cardioplegia showed that high extracellular $\mathrm{K}^{+}$levels during the ischenic episode may attribute to the improvement of the postischenic function [JYN95]

It thus can be concluded that there is a dual significance of the elements discussed above. First, the alterations in intra- and extracellular concentrations of these elements may be used as an indicator of injury inflicted on the myocardial cell and its membrane during ischemia and reperfusion. Secondly, these elements may play an important role in the induction of damage or, conversely, in the protection against ischemic injury. 


\subsection{Techniques to analyse elemental concentrations in cardiac tissue, with special reference to the proton microprobe}

In case elemental concentrations are to be determined at the cellular level in tissue sections the following demands must be met by the analytical technique used. First, the spatial resolution of the technique must be sufficient to distinguish individual myocardial cells. Second, the technique should provide the possibility to determine elements having a low concentration. There are a variety of techniques available to determine elemental concentrations in biological material. However, in case the elemental concentrations are to be determined at the cellular level only few microanalytical techniques are remaining, e.g., Nuclear Microscopy, Electron Probe Micro Analysis (EPMA), Ellectron Energy Loss Spectroscopy (EELS), Secondary Ion Mass Spectroscopy (SIMS), Laser Microprobe Mass Spectrometry (LMMS) and fluorescence microscopy.

Results from other studies [LEG80, MOR91] showed the promising potentials of nuclear microscopy with respect to the analysis of $\mathrm{Na}, \mathrm{Mg}, \mathrm{K}$ and $\mathrm{Ca}$ at the cellular level. In the study described in this thesis this technique is employed to study the alterations in elemental concentration in healthy and injured cardiac tissue. Nuclear microscopy or nuclear microprobe analysis comprises the techniques Particle Induced X-ray Emission (PIXE), Rutherford Backscattering (RBS) and Scanning Transmission Ion Microscopy (STIM). These techniques use the detection of characteristic X-rays (PIXE), the energy of scattered particles (RBS) and the energy loss of transmitted particles (STIM) after interaction of the sample with a scamning micron sized beam of charged particles (protons or alpha particles). Some of them or a combination of these techniques yield quantitative elemental analysis with sensitivities at the $\mathrm{mg}^{\mathrm{kg}} \mathrm{kg}^{-1} \mathrm{dry}$ weight (or ppm $=$ parts per million) level and identification of structural features at the microscale. As the name of nuclear microprobe analysis covers a wide range of used particles, it has to be emphasized that in this thesis only protons were used to bombard the sample and therefore the name of proton microprobe will be adopted.

Since PIXE is based upon the detection of characteristic X-rays emitted upon ionisation of individual atoms, no information is obtained about the molecular structure of the sample and no distinction can be made between free and bound atoms. Therefore, it should be noted that with PIXE the total amount of element present within the tissue is determined. As a result this technique can be used to determine the total cellular coment ${ }^{1}$ of the elements of interest. This implies that, although the sensitivity of PIXE is not sufficient to assess for example the free cytosolic $\mathrm{Ca}^{2+}$ concentration (see Table 1.1), this technique can be used to study the total cellular content of this element. This total cellular content is made up by the sum of the elemental content of subcellular organelles and compartments, such as myofibrils, SR and mitochondria. In addition,

${ }^{1}$ It is inportant to move that sowne terwinological differences exist in physics and phigsiology concerwing "the concentration of an element". In physics concentration is expressed in term:s of mass (c.g. $\mathrm{mg}$ element/ $\mathrm{kg}$ tolal diy mass., whereas in plysiology concentration refers to the amount of elenem present in an aqueous compantmen (a.g. mmol element f l cell water). In wiss thesis the term concentration is used for the anount of element present in solution in aqueous space, whereas the tern content is used in all other cases. 
in case PXE is employed using a proton beam focused to anicron-sized spot, also elements that are locally accumulated within the intracellular compartment can be determined.

\subsection{Aim of the study and outline of thesis}

The aim of the present study is to explore the possibilities of the Eindhoven proton microprobe set up to study the alterations in elemental concentration of healthy and damaged rat hearts at the cellular level. This study can be divided into a technological and a physiological part. A feasibility study has been performed to find out whether it was technically possible to measure the cellular content of a number of elements of interest, such as $\mathrm{Na}, \mathrm{Mg}, \mathrm{K}$ and $\mathrm{Ca}$, and to localize individual cells within the sample. The proton microprobe has been used to measure alterations in elemental content in rat hearts subjected to damaging conditions, such as ischemia/reperfusion and calcium paradox experiments. The physiological part of the study again can be subdivided. In the first place the differences in elemental content in healthy and damaged tissue have to be deternined. Secondly, the question whether cell-to-cell differences in elemental content due to the ischemic insult exist (cellular heterogeneity) is of special interest. It is known that part of the cells is damaged irreversibly after a certain period of ischemia, whereas other cells are still viable. Moreover, it is also interesting to study whether heterogeneity in mean cellular content can be observed in healthy cells. Finally, if the spatial resolution of the proton microprobe is sufficiently good, intracellular heterogeneity can be studied in both healthy and damaged nyocardial cells.

The basic aspects of the three most commonly used techniques in proton microprobe analysis, i.e, PIXE, RBS and STIM, are presented in Chapter 2. The calculation of the elemental content and the lowest detectable mass fraction obtainable with PLXE are also discussed in this chapter. Chapter 3 is devoted to the practical comparison between the proton microprobe and the electron microscope, since both instruments show close analogy. The difference in sensitivity between the two techniques is emphasized by experimental determination of the limit of detection for callum using both PIXE and EPMA. In Chapter 4 the adaptations and improvements in experimental procedures and instrumentation of the Endhoven proton microprobe are discussed to study thin tissue sections from rat hearts. Subsequently, the sample preparation procedures and the preliminary results of proton microprobe analysis of thin tissue sections from control, nomoxic, ischemic and reperfused rat hearts are discussed (Chapter 5). This study was performed to verify whether the measured values for the elemental content correspond with values from literature and gain insight into the possible artefacts introduced during the complete process of sample preparation. In a subsequent study the maximum alterations in elemental concentrations that are to be expected under severely damaging conditions were assessed. To this end the rat hearts were subjected to the so-called calcium paradox (Chapter 6). A more advanced study of hearts subjected to prolonged ischemia followed by reperfusion is given in Chapter 7 . In this chapter special attention is paid to the heterogeneity in cellular elemental content in damaged hearts, making use of the STMM technique to distinguish between individual myocardial cells. Finally the results of the whole study are discussed and some suggestions for improvement of the analysis of biological material using the proton microprobe are given in Chapter 8 . In this chapter also the advantages and disadvantages of the proton microprobe in biomedical research are summarized. 


\section{Chapter 2}

\section{Elemental analysis with X-rays; basic aspects}

\subsection{Introduction}

The main goal of our study is to determine changes in elenental concentration of $\mathrm{Na}, \mathrm{Mg}, \mathrm{K}$ and $\mathrm{Ca}$ in damaged myocardial tissue compared to healthy tissue. Since we are interested in the measurement of low concentration levels at the cellular level the analytical technique used must provide both a minimum detectable concentration at the $\mathrm{mg} / \mathrm{kg}$ level and a spatial resolution at the $\mu \mathrm{m}$-scale. Both demands are met by the PIXE (Particle Induced X-ray Emission) technique, which is based upon the detection of characteristic X-rays that are created during the bombardment of a target with a beam of energetic charged particles (usually protons in the $\mathrm{MeV}$ energy range). In case this particle beam is focused down to a diameter in the order of a few microns this technique is referred to as micro-PIXE. Because of the properties of micro-PIXE, a growing interest can be observed for using this techmigue to perform trace element anallysis of biological specimens at the cellular level [WAT95].

When a beam of $3 \mathrm{MeV}$ protons impinges on a specimen, several types of interactions can take place. In this chapter three different techniques, based upon the detection of characteristic $\mathrm{X}$-rays, scattered protons or transmitted protons are described:

i) Particle Induced X-ray Emission (PIXE), where the energy of the characteristic X-rays emitted from atomic inner shell transitions is measured, can be used to determine the amount of trace element present in the sample (in $\mathrm{ng} / \mathrm{cm}^{2}$ )

ii) Nuclear Back and Forward Scattering spectrometry (NBS and NFS), where the energy of elastically scattered protons is measured, can be used to determine the matrix composition and the local areal mass density of the sample (in $\mathrm{mg} / \mathrm{cm}^{2}$ ). It thus follows that the combination the results of PIXE and NBS/NFS analysis, i.e., the amount of trace element (in $\mathrm{ng} / \mathrm{cm}^{2}$ ) divided by the total areal mass density (in $\mathrm{mg} / \mathrm{cm}^{2}$ ), yields an elemental content with sensitivities down to the $\mathrm{ng} / \mathrm{mg}$ or parts per million (ppm) level.

iii) Scanning Transmission Ion Microscopy (STIM) can provide information on the structure and local areal mass density of the sample by measurement of the energy loss of the protons after transmission through the specimen.

In Sections 2.1 and 2.2 of this chapter the basic theory of these three techniques is treated. Subsequently, the calculation of the elemental concentration and the minimum detectable concentration obtainable with PIXE are discussed in Section 2.3.

Particle Induced X-ray Emission (PIXE) is an analytical technique based upon $X$-ray spectrometry, and was introduced in 1970 at the Lund Institute of Technology by Johansson and coworkers [JOH70]. In PIXE a sample is bombarded with a beam of charged particles (usually protons) in order to eject inner shell electrons from atoms in the sample. Within about 
$10^{-16}$ seconds the created vacancies will be filled by electrons from outer shells. During this process energy is released corresponding with the potential-energy difference between the two shells. The released energy is used for the emission of X-ray photons or Auger electrons characteristic for the ionized atom. Hence measurement of the energy of the $X$-ray allows identification of the element. The number of emitted X-rays is a measure for the number of atoms per unit area of the corresponding element in the sample under investigation.

Generally, vacancies can be created in several shells, thus giving rise to a large number of allowed transitions and hence to a large number of X-ray lines. X-rays emitted after creation of a $\mathrm{K}$-shell vacancy are called $\mathrm{KX}$-rays, while removal of electrons from the $\mathrm{L}$-shell is followed by the emission of $\mathrm{L} X$-rays. X-rays corresponding to $\mathrm{L}-\mathrm{K}$ transitions are called $\mathrm{K}_{\mathrm{a}}$ lines. The $K_{p}$-lines, with energies higher than the $K_{a t}$-lines, represent transitions to the $K$-shell from higher shells. The $K_{\alpha}$-lines are more intense than the $K_{\beta}$-lines and the $K_{\beta} / K_{\alpha}$ intensity ratio is characteristic for each element of the periodic system.

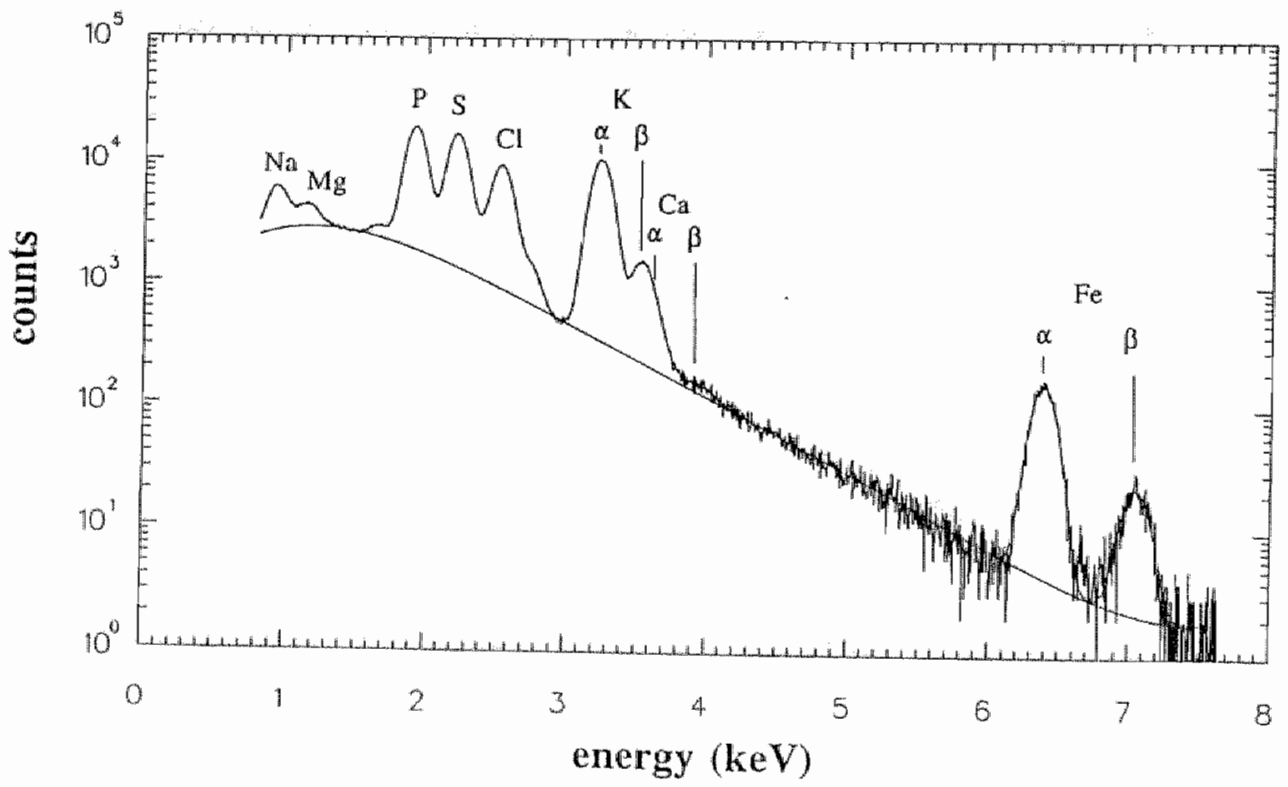

Figure 2.1: Example of a PLE spectrum, obtained during the irradiation with $3 \mathrm{MeV}$ protons of a rat myocardial tisste section. Tissue section thickness was 10 , $\mathrm{mm}$. The characteristic $X$-ray peaks of $N a, M g, P, S, C l, K, C a$ and Fe are superimposed on a continuous background. For $K$. Ca and $F e$ boh $K_{*}$ and $K_{p}$ peaks are wisible in the $X$-ray specrnum. The $K-K_{p}$ peak and the $C a-K_{\alpha}$ peak show a considerable overlap.

Lithium-drifted silicon $(\mathrm{Si}(\mathrm{Li}))$ detectors are generally used to directly measure the energy of the X-rays. These detectors provide the possibility to measure X-ray lines with an energy 
ranging from 1-20 keV simultaneously with a high efficiency and relatively good energy resolution $\left(\sim 150 \mathrm{eV}\right.$ for the $\mathrm{Mn}-\mathrm{K}_{\mathrm{\alpha}}$ line of $\left.5.895 \mathrm{keV}\right)$. A typical PIXE spectrum of a rat heart sample is given in Figure 2.1. The PIXE spectrum consists of the characteristic X-ray peaks of $\mathrm{Na}, \mathrm{Mg}, \mathrm{P}, \mathrm{S}, \mathrm{Cl}, \mathrm{K}, \mathrm{Ca}$ and $\mathrm{Fe}$ superimposed on a continuous background, which is strongly energy dependent (see also Section 2.3). The area of each peak is a measure for the amount of the corresponding element present in the irradiated area of the sample.

In some cases determination of the peak areas can seriously be hampered by peak overlaps. For $20<Z<30$ often peak overlaps between the $K_{r}$-line of element $Z$ and the $K_{p}$-line of element Z-1 occurs, like the overlap of the $\mathrm{K}-\mathrm{K}_{\beta}$ and the $\mathrm{Ca}-\mathrm{K}_{\alpha}$ lines in this particular X-ray spectrum. This overlap can be attributed to the small energy difference between these lines compared to the detector resolution of the $\mathrm{Si}(\mathrm{Li})$ detector.

In micro-PIXE analysis of biological tissue, usually thin $(1-3 \mu \mathrm{m})$ freeze-dried cryosections are used, especially if one is interested in elemental concentrations on a cellular level. However, PIXE analysis of bulk (thick) samples can also provide very useful information in biomedical studies [PIN93]. To study gross effects, PIXE can be used to determine elemental concentrations in bulk samples, followed by micro-PIXE analysis of thin sections to obtain information on the cellular level. In the following section the PIXE theory is described starting from the equations that are used for the calculation of elemental concentrations in thick targets (TTPIXE). It is shown that even in biological samples of $\mu \mathrm{m}$ thickness for several elements of interest thick-target effects have to be taken into account. Subsequently, simplifications of this theory for thin samples and samples of intermediate thickness are discussed.

\subsection{Particle Induced X-ray Emission}

\subsubsection{General description of thick target PIXE}

In this section a short, general description of thick target PIXE (TTPIXE) is given. An extensive treatment of the thick-target effects as described above is beyond the scope of this thesis and can be found in [MUN94]. However, the main purpose of this section is to present the TTPIXE theory in such a way that the physical interpretation becomes more transparent, specially to those not familiar with the PIXE theory.

The probability of creating an inner shell vacancy in the $\mathrm{K}$-shell of an elements with atomic number $Z$ is called the ionization cross section $\sigma_{Z, K}{ }^{i o n}\left(E_{p}\right)$, which is a function of the proton energy $E_{p}$. The protons loose energy on their way through the sample, mainly because of the Coulomb interaction of the bombarding protons with bound atomic electrons. As a result the ionization cross section $\sigma_{z K}$ ion $\left(E_{p}\right)$ becomes a function of the depth $x$ in the sample. The energy loss per unit length for a projectile passing through a medium of density $\rho_{s}\left(\mathrm{~g} / \mathrm{cm}^{3}\right)$ is called the mass stopping power and it is defined as

$$
S_{r}\left(E_{p}\right)=\frac{1}{\rho_{S}} \frac{d E_{p}}{d x}
$$


where $\left(d E_{p} / d x\right)$ is the energy loss of the protons along their path in the sample. The mass stopping power is energy dependent and is expressed in $\mathrm{keV} /\left(\mathrm{g} / \mathrm{cm}^{2}\right)$. The change in the ionization cross section due to the energy loss of the protons is one of the thick-target effects.

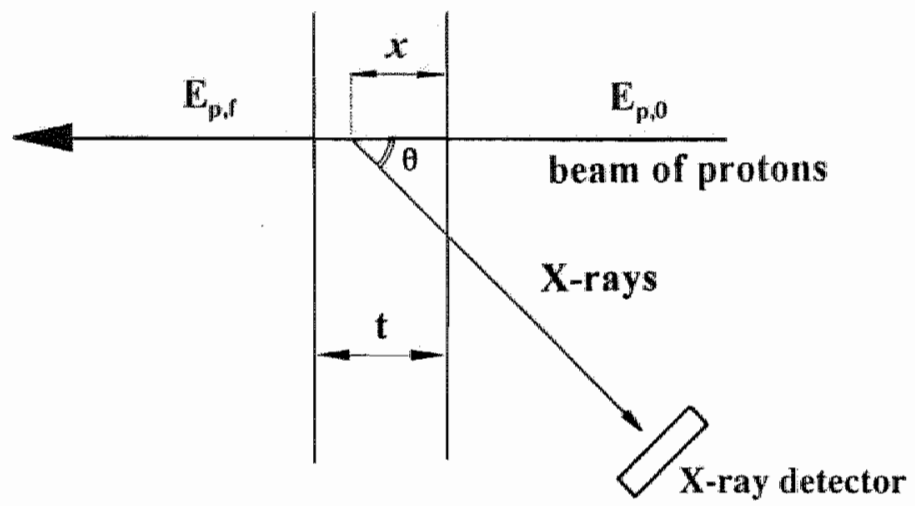

Figure 2.2: Schematic representation of the production of characteristic X-rays in a sample with thickness t. The initial proton energy is $E_{p, 0}$ while $E_{p, f}$ is the proton energy after transmission through the sample. X-rays produced at a depth $x$ in the sample have to travel a distance $x / \cos \theta$ through the sample in the direction of the detector.

Another thick-target effect is due to the absorption of X-rays, produced at a certain depth $x$, in the sample itself. Hence these $\mathrm{X}$-rays cannot reach the X-ray detector. Different materials absorb X-rays to different degrees and are characterized by their (mass) absorption coefficient. $\mathrm{X}$-rays travelling through a material of density $\rho_{s}$ suffer absorption to the degree

$$
\frac{I_{t}}{I_{0}}=\exp \left\{-\frac{\mu_{t}\left(E_{X Z}\right)}{\rho_{S}} \frac{\rho_{S} x}{\cos \theta}\right\}
$$

with

$I_{t} \quad=\quad$ transmitted X-ray intensity

$I_{0}=$ original X-ray intensity

$\mu_{t}\left(E_{X^{2}}\right)=$ linear absorption coefficient $\left(\mathrm{cm}^{-1}\right)$

$(x / \cos \theta)=$ distance travelled by the $\mathrm{X}$-ray in the sample in the direction of the detector $(\mathrm{cm})$.

The density of the absorbing material is introduced in this formula, since in literature usually the mass absorption coefficient $\left(\mu_{t} / \rho_{S}\right)$ is given. $\mathrm{X}$-rays of higher energy from heavier elements are less easily absorbed than low energy $\mathrm{X}$-rays. For this reason the transmission probability of $\mathrm{X}$-rays through a given medium is dependent on the $\mathrm{X}$-ray energy $E_{X}$. In addition, the transmitted $\mathrm{X}$-ray intensity depends on the depth $x$ from which the $\mathrm{X}$-rays have to travel, and thus depends on the proton energy $E_{p}$ as well. 
In summary, it can be concluded that both ionization cross section and transmission probability of $X$-rays through the sample depend on the proton energy $E_{p}$ and thus on the depth $x$ in the sample. As a result both ionization cross section and transmission probability have to be integrated over the depth in the sample. Since the ionization cross section is known as a function of $E_{p}$ and not of the depth $x$ in the sample, it is necessary to transform the integral over the depth $x$ in the sample to an integration over $E_{p}$. The relation between the depth $x$ in the sample and the proton energy $E_{\psi}$ follows from Eq. 2.1 and is given by:

$$
x=\int_{E_{p, \Theta}}^{E_{p}} \frac{1}{\rho_{s} S_{i}\left(E_{p}\right)} d E_{p}^{*}
$$

When thick-target effects are taken into account, i.e. the dependence of $\sigma_{z, x}{ }^{p w a}\left(E_{p}\right)$ and $T_{s}\left(E_{X z^{*}} E_{p}\right)$ on the proton energy Ep, the yield $Y\left(X_{Z}\right)$ has to be written in integral form. Using Eq. 2.3 for the transformation of the integration of $x$ to $E_{p}$, the yield $Y\left(X_{y}\right)$ of element $Z$ (the net peak content of this element in the $\mathrm{X}$-ray spectrum) due to the passage of $N_{p}$, protons is given by

$$
Y\left(X_{Z}\right)=N_{p} \frac{N_{a v}}{M_{Z}} c_{Z} \mathbb{e}_{Z}\left(E_{X_{Z}}\right) T_{a}\left(E_{X_{Z}}\right) \frac{\Omega}{4 \pi} \int_{E_{p_{i}, 0}}^{E_{p r}} \frac{T_{s}\left(E_{X_{Z}, E_{p}}\right) \sigma_{Z, X}^{p r o d}\left(E_{p}\right)}{S_{t}\left(E_{p}\right)} d E_{p}
$$

with

$$
T_{s}\left(E_{X_{z}}, E_{p}\right)=\exp \left(-\frac{\mu_{r}\left(E_{X_{z}}\right)}{\rho_{s} \cos \theta} \int_{E_{p_{0}}}^{E_{p}} \frac{d E_{p}^{\prime}}{S_{i}\left(E_{p}^{\prime}\right)}\right)
$$

and with

$N_{p} \quad=$ number of particles impinging on the target

$N_{a, \quad} \quad$ Avogadro's number $\left(\mathrm{mol}^{-1}\right)$

$M_{3} \quad=$ atomic mass of element $Z(\mathrm{~g} / \mathrm{mol})$

$c_{z} \quad=\quad$ mass fraction (concentration) of element $Z$

$\varepsilon_{z}\left(E_{X}\right)=$ detector efficiency, i.e., the probability that the energy of an X-ray with energy $E_{X z}$ is completely absorbed in the detector crystal

$T_{a}\left(E_{X j}^{*}\right)=$ transmission probability of $X$-rays with an energy $E_{X ; z}$ through an absorber $Q / 4 \pi=$ relative detector solid angle

$\sigma_{Z, X^{\text {prot }}}\left(E_{p}\right)=X$-ray production cross section of element $Z\left(\mathrm{~cm}^{2}\right)$

$S_{s}\left(E_{j}\right)=$ stopping power $\left(\mathrm{keV} \mathrm{cm} \mathrm{cm}^{2} / \mathrm{g}\right)$

$T_{s}\left(E_{X X^{\prime}} E_{p}\right)=$ transmission probability of $X_{\text {-rays }}$ with an energy $E_{X^{2}}$ through the sample in the direction of the $X$-ray detector 


$\begin{array}{ll}\rho_{S} & =\text { density of the sample }\left(\mathrm{g} / \mathrm{cm}^{3}\right) \\ \theta & =\text { emission angle of X-rays, angle between the sample normal and the } \\ & =\text { direction of the X-ray detector } \\ E_{p_{0} \theta} & \text { proton energy before transmission through the sample }(\mathrm{keV}) \\ E_{B_{v}} & =\text { proton energy after transmission through the sample }(\mathrm{keV}) \\ \mu_{i}\left(E_{X S}\right) & =\text { total linear X-ray absorption coefficient of the sample }\left(\mathrm{cm}^{-1}\right) .\end{array}$

The cross section for the production of an X-ray can be written as:

$$
\sigma_{Z, X}^{p r o d}\left(E_{p}\right)=\sigma_{Z, K}^{\text {ion }}\left(E_{p}\right) \cdot \omega_{Z, K} \cdot b_{Z, X(K)}
$$

with
$\sigma_{z, K}{ }^{i c n}\left(E_{p}\right)=$ cross section for ionization of the K-shell of element $Z$
$\omega_{Z K K} \quad=$ fluorescence yield for the $\mathrm{K}$-shell; this is the probability of a vacancy being filled by an electron from a higher shell under emission of an $X$-ray
$b_{Z x(K)}=\quad$ branching ratio; the fraction of total $K$-shell $X$-rays in the line $X_{Z(K)}$.

To shorten the notation the absolute detection efficiency $\varepsilon_{a b s}$ is introduced:

$$
\varepsilon_{a b z}\left(E_{X Z}\right)=\varepsilon_{z}\left(E_{X}\right) T_{a}\left(E_{X Z}\right) \frac{\Omega}{4 \pi}
$$

The relation between the mass fraction (concentration) of element $Z$ and the number of atoms per $\mathrm{cm}^{2} N_{Z}$ in the sample is given by:

$$
c_{z}=\frac{M_{z} N_{z} t}{N_{\alpha w} \rho_{S} t}
$$

In principle, the integral over $E_{p}$ in Eq. 2.4 can be solved, although only numerically, when

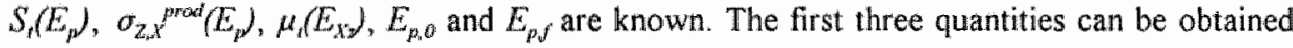
from literature, while the last two quantities have to be determined experimentally. Values for both stopping power and X-ray absorption coefficient can be found in literature for single elements. For compound samples, such as biological tissue, the values of these two quantities can be found by summation of the contributions of the pure elements. According to the phenomenological model postulated by Bragg and Kleeman [BRA05] the stopping power of a compound sample can be calculated using

$$
S_{i}\left(E_{p}\right)=\sum_{j} c_{j} S_{j}\left(E_{p}\right)
$$


where $c_{j}$ is the mass fraction of element $\mathrm{j}$ in the sample and $S_{j}\left(E_{p}\right)$ is the mass stopping power of element $\mathrm{j}$. In this model, commonly known as Bragg's rule, the contribution of the atomic cores (electrons in closed shells) and of atomic bonds (valence electrons) to the stopping power are not taken into account. As a result large deviations from Bragg's rule are to be expected for samples containing molecules with strong chemical bonds. For this reason Ziegler and Manoyan have proposed the cores-and-bonds (CAB) model to calculate the stopping power for organic materials [ZIE88]. Near the proton energy where the stopping power is maximum $\left(E_{p} \approx 0.075 \mathrm{MeV}\right)$ the deviations are largest and a difference between Bragg's rule and the CAB-model of about $20 \%$ was found. However, a recent study of Wallace and coworkers [WAL94], who compared experimental data on the hydrogen stopping powers in polystyrene with those calculated using both Bragg's rule and the $\mathrm{CAB}$-model, shows that Bragg's rule is accurate within $2 \%$ for proton energies above $1 \mathrm{MeV}$. As we start with a beam energy of $3 \mathrm{MeV}$ in this investigation, it was decided to apply Eq. 2.9 .

Identical to the mass stopping power the mass absorption coefficient for a compound sample can be calculated using the weighted average of the mass absorption coefficients of pure elemental targets:

$$
\frac{\mu_{t}\left(E_{X Z}\right)}{\rho_{S}}=\sum_{j} c_{j} \frac{\mu_{j}\left(E_{X_{Z}}\right)}{\rho_{j}}
$$

with $\left(\mu_{j}\left(E_{X j}\right) / p_{j}\right)$ the mass absorption coefficient of element $j$. To apply Eqs. 2.9 and 2.10 the concentration of the matrix elements in the sample must be known. Moreover, the value of the proton energy after passage through the specimen $E_{p, f}$ can only be callculated for a sample with known thickness (areal mass density) and composition. To determine the sample thickness the STIM technique can be applied, while NBS and NFS can be used if additional information on the sample composition is required. More information on both techniques can be found in Section 2.2 .

In the case of intermediate thick targets it is possible to make corrections for thick target effects without having to solve the integral in Eq. 2.4 numerically. This is only valid if it is possible to approximate the cross section at every depth in the sample by an average value

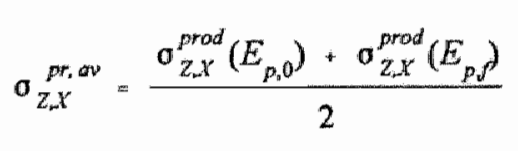

From Eqs. 2.1 and 2.9 it follows that the transformation of the energy range to the sample thickness depends on the sample composition. Using Eq. 2.3 to transform the integral over $E_{p}$ to an integral over $x$, Eqs. 2.4 and 2.5 can be solved analytically: 


$$
\begin{aligned}
Y & =N_{p} \frac{N_{a v}}{M_{z}} c_{z} \sigma_{Z z X}^{p r a v} \varepsilon_{a b s}\left(E_{X_{z}}\right) \rho \cdot \int_{0}^{t} \exp \left(\frac{\mu_{t}\left(E_{X_{z}}\right)}{\rho_{z} \cos \theta} \rho_{s} x\right) d x \\
& =N_{P} \frac{N_{a w}}{M_{z}} c_{z} \sigma_{Z X}^{p r a v} \varepsilon_{a b s}\left(E_{X_{z}}\right) \frac{\cos \theta}{\mu_{t}\left(E_{X_{z}}\right) / \rho_{s}}\left(1-T_{z}\left(E_{X_{z}}, t\right)\right)
\end{aligned}
$$

If the sample is so thin that the energy loss of the protons is very low (and thus $\sigma_{z, t^{p}}^{\text {prod }}\left(E_{p, 0}\right) \approx$ $\sigma_{z, X}{ }^{\text {prod }}\left(E_{p, p}\right)$ throughout the sample) and the absorption of $\mathrm{X}$-rays within the sample itself can be neglected $\left(\left(T_{S}\left(E_{X_{x}} E_{p}\right) \approx 1\right)\right.$, the well-known formula for thin targets can be derived from Eq. 2.12 by Taylor expansion of $T_{5}\left(E_{X}, t\right)$ about $t=0$ :

$$
Y\left(X_{Z}\right)=N_{p} \frac{N_{a v}}{M_{Z}} c_{Z} \rho_{S} t \sigma_{Z, X}^{p r o d}\left(E_{p, 0}\right) \varepsilon_{a b s}
$$

Although both Eqs. 2.4, 2.12 and 2.13 can be used to calculate elemental concentrations in biological tissue, it is useful to determine the influence of thick target corrections on the final result as a function of X-ray energy, sample composition and target thickness both theoretically and experimentally. This provides the possibility to verify the usefulness of the presented formulas and it enables one to predict under which circumstances which formulas can be applied.

\subsubsection{Theoretical evaluation and experimental verification of thick target PIXE for light elements in biological samples}

The matrix correction factor (MCF) can be defined as the ratio of the elemental concentrations calculated with and without thick target corrections respectively. Hence the MCF can be calculated using Eqs. 2.4 and 2.13. Since Eq, 2.4 cannot be solved analytically, the integration over $E_{\mathrm{p}}$ is performed numerically using the computer program PANEUT (PIXE Analysis Eindhoven University of Technology), written by Munnik [MUN94]. A more comprehensive description of this program and its data base is given in Section 4.3.

Figure 2.3 shows the matrix correction factor for $\mathrm{Na}, \mathrm{Mg}, \mathrm{K}$ and $\mathrm{Ca}$ as a function of the total areal mass density of the sample. To mimic biological tissue a $100 \%$ carbon matrix is used for the calculations. As expected the influence of thick-target effects is the most 
pronounced for $\mathrm{Na}$ and $\mathrm{Mg}$. For $\mathrm{Na}$ the MCF amounts to 1.46 for samples with an areal mass density of $0.3 \mathrm{mg} / \mathrm{cm}^{2}$, corresponding to an absorption of $\mathrm{X}$-rays of about $31 \%$. For samples with $\rho_{s} t=0.6 \mathrm{mg} / \mathrm{cm}^{2}$ neglecting thick-target effects would even result in an underestimation of the $\mathrm{Na}$ concentration by a factor of 2 . On the other hand, for the same sample thickness the Ca thick-target concentration differs from the thin-target concentration by only $3 \%$.

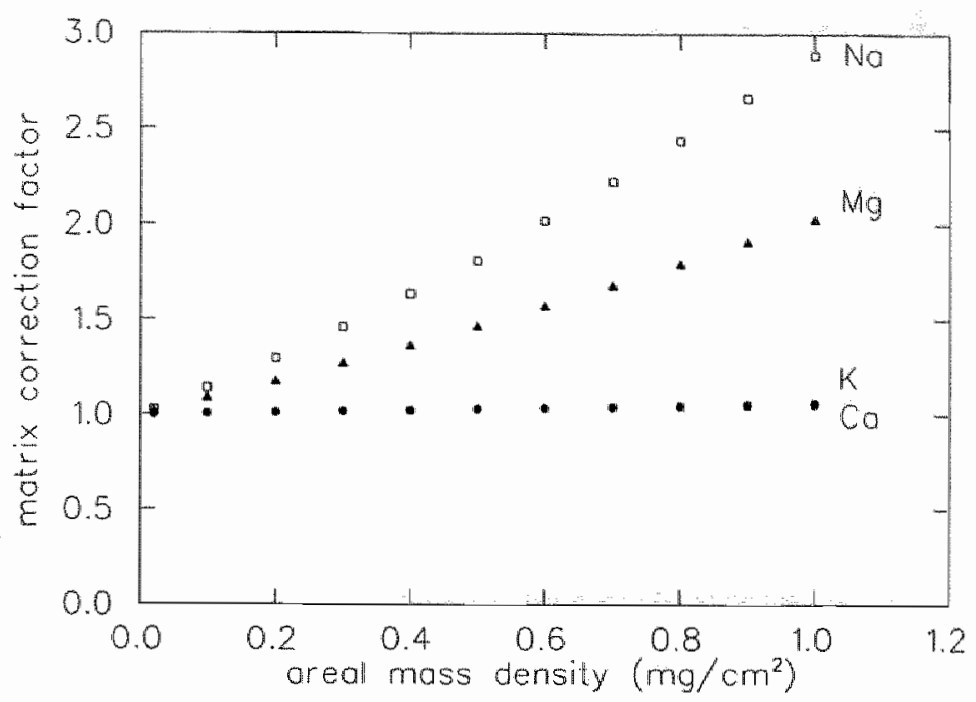

Figure 2.3: Theoretical marrix correction facror $(M C F)$ for several elements as a function of areal mass density of a carbon sample. The MCF was calculated using Eq. 2.14, assuming that the characteristic $X$-rays of $\mathrm{Na}, \mathrm{Mg} . \mathrm{K}$ and $\mathrm{Ca}$ had to pass through a $100 \%$ carbon matrix.

In our study of damaged myocardial tissue in most cases sections with a thickness of about $10 \mu \mathrm{m}$ were used. After subsequent freeze-drying the areal mass density of these sections ranged from 0.15 to $0.4 \mathrm{mg} / \mathrm{cm}^{2}$. From Figure 2.3 it therefore can be concluded that in this case for light elements $(\mathrm{Na}, \mathrm{Mg})$ thick-target effects cannot be neglected.

The large value for the MCF for $\mathrm{Na}$ and $\mathrm{Mg}$ can be contributed almost completely to the absorption of X-rays in the sample instead of changes in the ionization cross section due to slowing down of the protons. This can be shown as follows. If the proton energy degradation is neglected, the MCF is given by the ratio of Eqs. 2.12 and 2.13,

$$
M C F=\frac{\rho_{S} t}{\frac{\cos \theta}{\left(\mu_{t} / \rho_{S}\right)}\left(1-T_{S}\left(E_{X_{Z}}, t\right)\right)}
$$


which can be solved analytically. Using this formula for $N$ a in a $1.0 \mathrm{mg} / \mathrm{cm}^{2}$ carbon matrix the calculated MCF is $0.5 \%$ higher as compared to Eq. 2.4. For the same areal mass density for Ca a 1.6\% lower value is found. This can be explained by the fact that for $\mathrm{Na}$ the maximum ionization cross section is reached for $E_{p}<3 \mathrm{MeV}$, while for heavier elements the cross section decreases for $\mathrm{E}_{\mathrm{p}}<3 \mathrm{MeV}$.

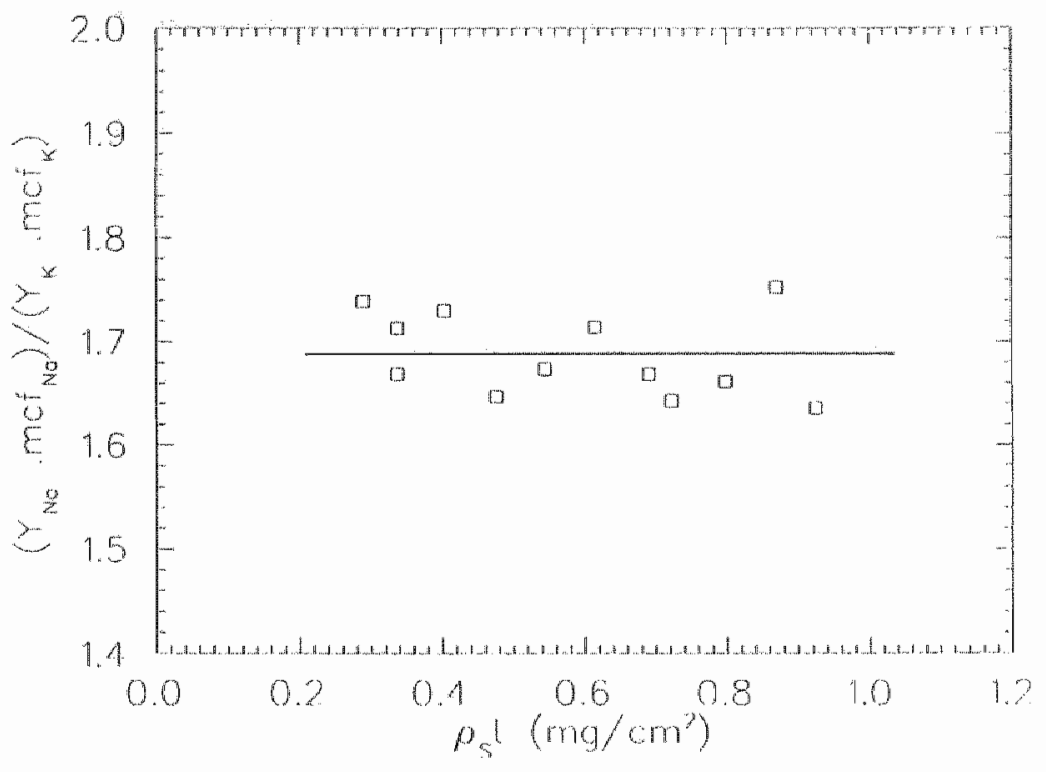

Figure 2.4: Experimenral valialarion of calculated matrix correction factors for $\mathrm{Na}$ and $K$ in an organic (polyethylene glycol) matrix. The ratio of the PLXE yields of $N a N_{\text {WW }}$ and $K\left(Q_{k}\right)$ both corrected for thick target effects (i.e., multiplied by $M C F_{N_{0}}$ and $M C F_{N}$ ). should be independent of the areal mass density $\rho_{s}$ t of the sample. Both $Y_{N a}$ en $Y_{k}$ were determined from the X-ray spectra obtained during proton bombardment of PEG samples, whereas the matrix correction factors were calculated using Eq. 2.14.

An experimental validation for the matrix correction factors was obtained using samples with a known and homogeneous concentration distribution. Known amounts of $\mathrm{NaCl}$ and $\mathrm{KCl}$ (both from Merck, Darmstadt, Germany) were added to $20 \%$ (w/v) polyethylene glycol (PEG, M.W. 18,500, Polyscience No. 4686) stock solution. A droplet of the PEG-salt mixture was frozen in melting isopentane to allow sectioning. Sections were cut in a cryostat microtome at $-20^{\circ} \mathrm{C}$ at a thickness ranging from 10 to $50 \mu \mathrm{m}$. The sections were collected on thin $(\approx 100 \mathrm{~nm})$ Formvar foils and subsequently freeze-dried at ambient temperature. The added amounts of mineral salts in the freeze-dried sections amounted to about $10,000 \mathrm{ppm} \mathrm{Na}$ and $10,000 \mathrm{ppm} \mathrm{K} \mathrm{dry} \mathrm{weight.} \mathrm{The} \mathrm{areal} \mathrm{mass} \mathrm{density} \mathrm{of} \mathrm{the} \mathrm{freeze-dried} \mathrm{sections} \mathrm{ranged} \mathrm{from}$ about $0.2-0.3 \mathrm{mg} / \mathrm{cm}^{2}$ for sections cut at $10 \mu \mathrm{m}$ thickness to about $1.0 \mathrm{mg} / \mathrm{cm}^{2}$ for the 
thickest sections. All samples were analyzed using a scanning proton beam with a spot size of $7 \times 7 \mu \mathrm{m}^{2}$ and $250 \mathrm{pA}$ beam current until a net peak content of about 10,000 counts was obtained. To check whether the dissolved mineral salts were distributed uniformly; a large area of about $225 \times 225 \mu \mathrm{m}^{2}(32 \times 32$ pixels) was scanned by the proton beam. If no appreciable differences in $\mathrm{Na}$ and $\mathrm{K}$ content between the individual positions scanned by the proton beam were found, it was assumed that both $\mathrm{Na}$ and $\mathrm{K}$ were distributed homogeneously in the sample. In this case PIXE analysis of sections of various thickness should yield the same values for the $\mathrm{Na}$ and $\mathrm{K}$ concentration after correction for thick target effects. The results of the measurements is depicted in Figure 2.4. From this figure it can be concluded that the influence of thick target effects on the calculated elemental concentration is treated correctly by the PANEUT routine. Specially for light elements like Na this is very important if reliable quantitative results are to be obtained.

\section{Influence of matrix composition on thick target PIXE-corrections}

Munnik and coworkers [MUN94b] have calculated the influence of the composition of a matrix consisting completely of $\mathrm{C}$ and $\mathrm{O}\left(\mathrm{a} \mathrm{C}_{6} \mathrm{O}_{1-\tau}\right.$ matrix) on the thick target yield for light elements $(\mathrm{Na}-\mathrm{Cl})$, with $\mathrm{c}$ ranging between $0(100 \% \mathrm{O}$ matrix $)$ and $1(100 \% \mathrm{C}$ matrix). They found that the yield for light elements decreases by a factor of 2 for the change of a $100 \% \mathrm{C}$ matrix to a $100 \% \mathrm{O}$ matrix, mainly due to a significant change in the value of the mass absorption coefficient $\mu$. For high energy $\mathrm{X}$-rays from heavy elements the transmission is high (low value of $\mu$ ) and thus the effect of the change is less important.

It should be noted that in the study of Munnik et al. [MUN94b] infinitely thick samples were considered. For thinner samples, however, the influence of both the stopping power and the absorption coefficient on the matrix correction factor are expected to be less pronounced. Moreover, it is not likely that the matrix composition of a biological sample will show a large degree of heterogeneity. For this reason it is interesting to calculate the value of the matrix correction factor as a function of the matrix composition of the sample.

In our study on elemental distributions in damaged myocardial tissue freeze-dried cryosections were used. The contribution of the trace elements in the sample to the total areal mass density was neglected. From NBS analysis the following mean values for the mass fractions of the matrix elements in myocardial tissue were found: $c_{y f}=0.05, c_{c}=0.70$, $c_{o}=0.15$ and $c_{N}=0.10$. The carbon mass fraction was varied from $49 \%$ to $82 \%$. Since the mass fractions of the matrix elements should add up to $100 \%$ the mass fractions of $\mathrm{N}, \mathrm{O}$ and $\mathrm{H}$ were adjusted as well. For example, an increase of $c_{C}$ by $3 \%$ is counterbalanced by a decrease in concentration of $1 \%$ for the other matrix constiluents. For a change from an oxygen matrix to a carbon matrix the stopping power increases by about $6 \%$.

In Fig. 2.5 the $\mathrm{MCF}$ for $\mathrm{Na}, \mathrm{Mg}, \mathrm{K}$ and $\mathrm{Ca}$ is displayed as a function of the carbon mass fraction $c_{C}$. The sample areal mass density $\left(\rho_{s} t\right)$ is $1.0 \mathrm{mg} / \mathrm{cm}^{2}$. It appears that light elements are more sensitive to changes in the matrix composition. However, a decrease in $c_{C}$ from $80 \%$ down to $50 \%$ only results in a $5 \%$ increase in the $\mathrm{Na}$ matrix correction factor. This can be explained as follows: the influence of the mass stopping power $S_{\text {, on the }}$ MCF is rather small compared with the mass-absorption coefficient $\mu_{t} / p_{s}$. In addition, the matrix elements $C, N$ 
and $\mathrm{O}$ have almost similar values for $S_{p}$ and thus from equation 2.9 it follows that changing their respective mass fractions is of minor influence on the value of $S_{t}$ for the compound sample. Furthermore, the differences in the values of $\mu / \rho_{S}$ for the main sample constituents is of less importance for relatively thin specimens (see Eq. 2.14).
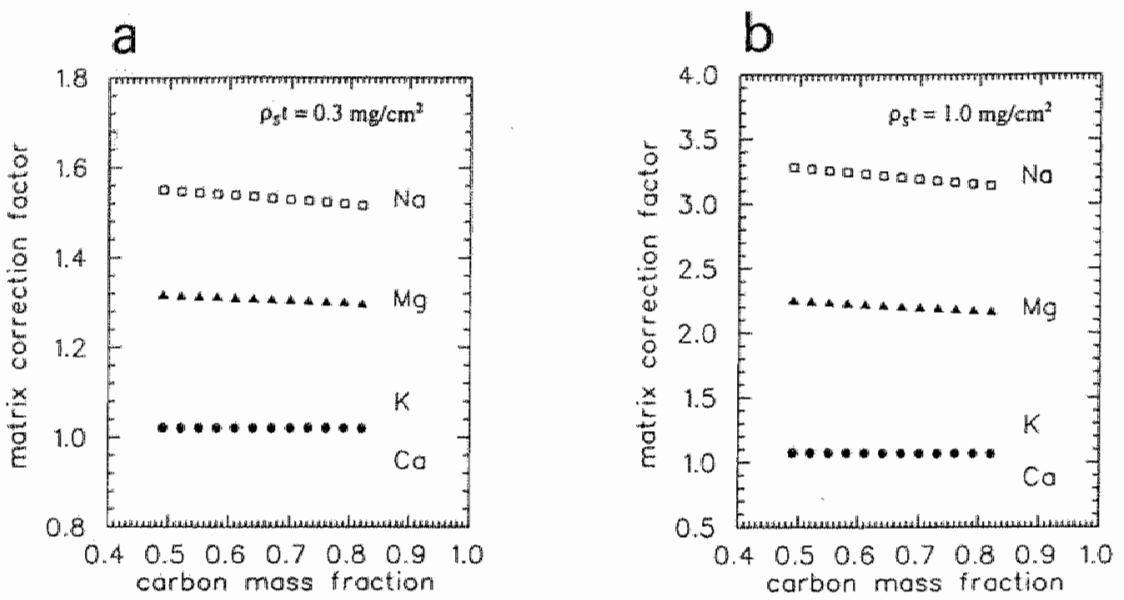

Figure 25: Theoretically determined influence of the carbon mass fraction on the matrix correction factor for $\mathrm{Na}, \mathrm{Mg}, \mathrm{K}$ and $\mathrm{Ca}$. The toral areal mass density $\left(\rho_{s} t\right)$ is kept constant at $0.3 \mathrm{mg} / \mathrm{cm}^{2}(a$.$) and 1.0 \mathrm{mg} / \mathrm{cm}^{2}(b$.$) .$

As a result it can be concluded that in most cases it suffices to determine the (local) thickness of the specimen and to use a mean matrix composition for the calculation of the concentration of trace elements in the sample. This circumvents the need for an exact determination of the concentration of the matrix elements for each position in the scanned area.

\subsection{Determination of the local areal mass density}

PIXE analysis is employed to determine the number of atoms per unit area for elements with $Z$ $z 11$. In case a scanning proton microprobe is used, PIXE can be applied to determine the amount of elements present in the sample for each position of the scanned area. However, local $X$-ray intensities often reflect local thickness variations. This effect can be accounted for by independent determination of the local areal mass density. Three different techniques can be used for mass normalization, namely:

i. nuclear scattering spectroscopy

ii. Scanning Transmission Ion Microscopy (STIM)

iii. normalization on the Bremsstrahlungs background in the PIXE spectrum. 
The third method has a number of disadvantages that can result in an overestimation of the areal mass density of the sample and thus can lead to too low values for the elemental content [MUT95, VIS85]. For this reason in the following sections the basic theory and some specific aspects of only nuclear scattering spectroscopy and STIM are presented, with special attention to their applicability in the analysis of biological tissue.

\subsubsection{Nuclear Back and Forward Scattering spectroscopy}

Nuclear Back (NBS) and Forward (NFS) Scattering spectroscopy is based upon the detection of the energy of protons after collision with the nuclei of atoms present in the bombarded sample. Under the assumption that the interaction between the protons and the target nuclei can be described by an ellastic two-body collision, the energy after the collision can be calculated from conservation of momentum and energy. The ratio of the proton energies after and before the collision is called the kinematic factor $K_{M t r}$

$$
K_{M_{t}}=\left(\frac{\sqrt{1-\left(m_{p} / M_{t}\right)^{2} \sin ^{2} \theta}+\left(m_{p} / M_{t}\right) \cos \theta}{1+\left(m_{p} / M_{t}\right)}\right)^{2}
$$

which depends on the ratio of the proton to the target mass $\left(m_{p}, M\right)$ and the scattering angle $\theta$. After the collision the protons will have an energy $K_{M t} E_{p, 0}$ As a result, protons scattered from different elements have different energies, and thus the elements present in the sample can be identified by determination of the energy of the scattered protons if $E_{p, 0}$ and $\theta$ are known.

The probability that a proton is scattered at an angle $\theta$ into a solid angle $d \Omega$ is called the differential scattering cross section $(d \sigma / d \Omega)$, where $d \Omega$ is taken so small that the scattering angle $\theta$ is well defined. The average differential scattering cross section $\sigma(\theta)$ over the field of view of the particle detector with a solid angle $\Omega$ then is given by:

$$
\sigma(\theta)=\frac{1}{\Omega} \int_{\Omega}(d \sigma(\theta) / d \Omega) d \Omega
$$

For very small detector solid angles $\sigma(\theta)-(d \sigma(\theta) / d \Omega)$. The probability of scattered protons falling within a finite solid angle $\Omega$ around scattering angle $\theta$ now is given by $\sigma(\theta) \cdot \Omega$.

The differential scattering cross section $(d \sigma(\theta) / d \Omega)$ depends on the mass and charge of both the projectile and target, the projectile energy $E_{p}$ and the scattering angle $\theta$. This quantity can be calculated for each element using Rutherford's formula [RUT11]. However, above a proton energy of about $1 \mathrm{MeV}$ elastic nuclear scattering of protons by light nuclei ( $\mathrm{C}, \mathrm{N}, \mathrm{O}$, $\mathrm{H}$ ) in addition to Coulomb interaction can occur. This results in a deviation of the scattering cross sections from their Rutherford values. 
A typical example of a backscattering spectrim of a biological tissue section is depicted in Figure 2.6. In this case the analyzed specimen contains more elements (compound sample) and thus the backscattering spectrum contains several peaks. Protons scattered from heavy-mass elements have higher energy than protons that collided with light elements, causing yield on the right side in the backscatter spectrum.

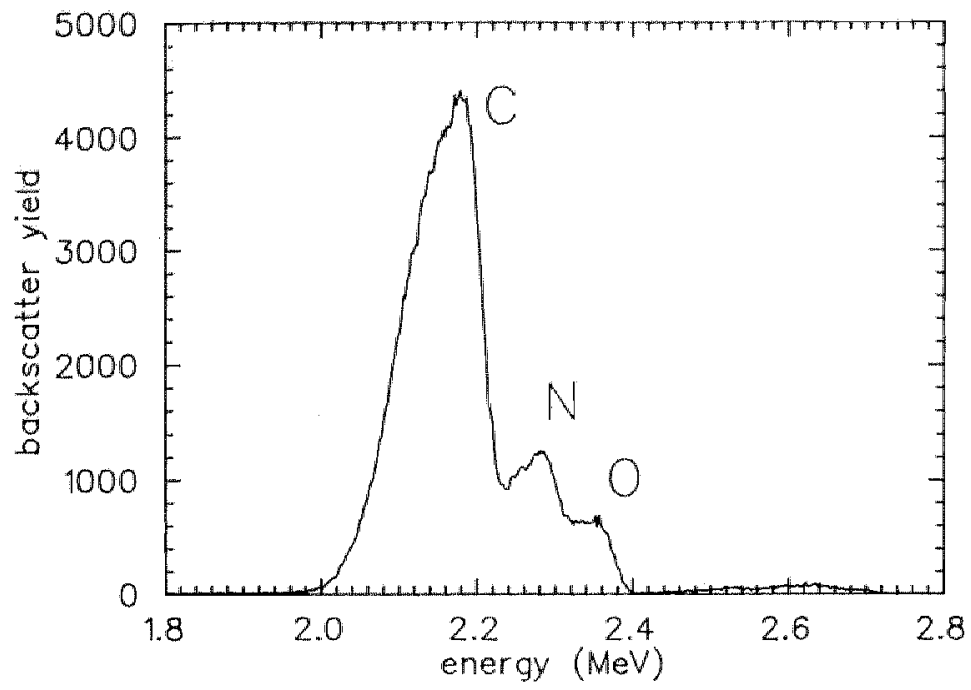

Figure 2.6: Backscatter spectrum obtained during the irradiation of a rat heart sample with 3 Me $Y$ protons. The corresponding peaks of the matrix elements $(C, N$ and $O)$ are resolved and clearly visible in the spectrum.

Protons that scattered from element $Z$ at the front surface of the sample are detected at an energy $K_{M r} E_{p, 0}$ When backscattering takes place at the back surface the protons additionally lose energy on their way through the sample both on the inward and outward path and is thus detected with an energy $E_{p, I}<K_{M i} E_{p, 0}$ Protons backscattered from the area between the two surfaces are detected with intermediate energies. The energy difference between the low and the high energy edge of a peak of element $Z$ in the NBS spectrum is proportional to the areal density of element $Z$. The total mass per unit area $\rho_{Z} t$ of element $Z$ present in the sample is proportional to the total backscatter yield for element $Z$ (i.e., the area under the peak in the NBS spectrum):

$$
Y_{N B S}(Z)=\frac{N_{p} \rho_{Z} t N_{\alpha w} \Omega_{N B S} \sigma_{z}^{N B S}\left(E_{p, 0}, \theta\right)}{M_{Z}}
$$


with

$N_{p} \quad=\quad$ number of protons imping on the target

$\rho_{Z} \quad=$ density of element $\mathrm{Z}\left(\mathrm{g} / \mathrm{cm}^{3}\right)$

i $\quad=$ sample thickness $(\mathrm{cm})$

$N_{\infty,} \quad=\quad$ Avogadro's number $\left(\mathrm{mol}^{-1}\right)$

$M_{Z} \quad=\quad$ atomic mass of element $\mathrm{Z}(\mathrm{g} / \mathrm{mol})$

$\sigma_{Z}{ }^{\mathrm{MBS}}\left(E_{p_{0}, 0} \theta\right)=$ average differential scattering cross section of element $\mathbb{Z}\left(\mathrm{cm}^{2} / \mathrm{sr}\right)$ at proton energy $E_{p, 0}$ and an angle $\theta$ with respect to the beam axis

$\Omega_{\text {NBS }} \quad=\quad$ solid angle of backscatter detector $(\mathrm{sr})$

This formula is valid under the assumption that the sample is so thin that the energy loss of the protons after passage through the sample can be neglected, i.e., $E_{p}=E_{p,}$ throughout the sample. Therefore the scattering cross section has the same value $\sigma_{z}^{A B S}\left(E_{0} \theta\right)$ everywhere in the sample. The superscript NBS is used to distinguish the scattering cross section from the ionization cross section $\sigma_{Z X}{ }^{i}\left(E_{p}\right)$. When the detector solid angle $\Omega_{\text {NBS }}$ and the total number of particles $N_{p}$ incident on the target are known, equation 2.17 can be used to calculate the areal mass density of element $Z$. The determination of the detector solid angle is discussed in Section 2.3.

The area under each peak in the NBS spectrum can easily be extracted from the spectrum if both the high and low energy edge are clearly identifiable. For biological material this is true for samples with an areal density $\rho_{s} t \leq 0.7 \mathrm{mg} / \mathrm{cm}^{2}$. For thicker samples the peaks start to overlap, thereby hampering the possibility to determine the yield for each individual element directly from the spectrum. In this case the areal density of the elements can be determined making use of the height of the peaks in the spectrum [CHU78].

From kinematics of the collision process it follows that backscattering of protons only can occur if $m_{p}<M_{r}$. For this reason the hydrogen areal density must be determined from the detection of forward scattered protons. For protons scattered in forward direction, Eq. 2.17 becomes:

$$
Y_{N F S}(Z)=\frac{N_{p} p_{Z} t N_{a v} \Omega_{N F S} \sigma_{Z}^{N F S}\left(E_{p, 0} \theta\right)}{M_{Z}}
$$

Eqs. 2.17 and 2.18 can be used to determine the areal mass density of all matrix elements present in the sample. The total areal mass density of the sample then simply follows from

$$
p_{S} t=\rho_{H} t+\sum_{i}\left(\rho_{i} t\right)
$$

where the subscript $i$ refers to the elements detected using NBS analysis. 
Soft tissue mainly consists of the organic elements $C, N, O$ and $H$. If the contribution of the elements with a mass fraction $<0.5 \%$ (trace elements) to the total areal mass density is neglected, the concentration of the matrix element $j$ is given by:

$$
c_{j}=\frac{\rho_{j} t}{\rho_{S} t}
$$

In conclusion back and forward scattering spectroscopy can be applied to determine both the areal mass density and the composition of the sample. The first can be used to normalize the results coming from PIXE analysis, while the latter is needed to calculate both the mass stopping power and the mass attenuation coefficient for a compound sample. Another important advantage of both NBS and NFS is that they can be executed simultaneously with the PIXE analysis. In case no information on the sample composition is required, the STIM technique can be employed by itself to determine the local thickness of the sample.

\subsubsection{Scanning Transmíssion Ion Microscopy}

During Scanning Transmission Ion Microscopy (STIM) analysis, a particle detector is used to measure the energy of the protons after passage through the specimen. This detector can either be placed directly behind the sample (bright-field STIM) or off-axis under a small angle with respect to the beam direction (dark-field STIM). In the bright-field mode a detector efficiency close to $100 \%$ is obtained, since almost each proton is detected. In this case a beam current of about $0.1-1 \mathrm{fA}$ has to be employed to avoid detector damage. Moreover, due to the high efficiency of detection higher beam currents would result in excessive count rates, since a beam current of $1 \mathrm{fA}$ already corresponds with 12,500 detected protons per second. The reduction of the beam current can be accomplished by a reduction of the object and aperture diaphragms in the microprobe set-up. As a result a smaller beam spot size and thus an improvement in the spatial resolution of the microprobe is expected for bright-field STIM compared to PIXE, where a beam with a diameter of about $3 \mu \mathrm{m}$ is employed. At present the smallest beam spot-size reported for STIM analysis is $50 \mathrm{~nm}$ [LEG93]. Theoretical estimations of the smallest beam spot size obtainable in our microprobe system during STIM experiments are presented in Section 4.2, together with some methods to determine the beam diameter experimentally.

After its introduction in 1983 [SEA83, OVE83], STIM primarily has been employed to obtain a two-dimensional image of the sample under investigation. This is probably due to the fact that the small spot size obtainable during STIM provides an excellent tool for high resolution imaging. Up to the time of writing this thesis only few reports on the use of STIM to determine the local areal mass density can be found in literature [LEF87]. In case STIM is employed for imaging purposes, the energy loss of the individual protons after passage through the sample is used as a contrast parameter. However, making use of Eq. 2.1 with known mass stopping power, the measured energy loss can be transformed into areal mass densities: 


$$
\int_{0}^{I} \rho d x=\int_{E_{p, 0}}^{E_{p p}}\left[\frac{d E}{d(\rho x)}\right]^{-1} d E
$$

This means that the STIM technique also can be employed to normalize the elemental maps obtained with PLXE. As will be explained bellow, the uncertainty in areal mass density deduced from the proton energy loss is determined by the precision of the energy loss measurement. The main uncertainties in the energy loss measurement are caused by the instrumental energy resolution and the (longitudinal) energy straggling.

The instrumental energy resolution is determined by the energy resolution of the particle detector and the energy spread of the incident proton beam. The resolution of the particle detector is typically about $12 \mathrm{keV}$ for the $3 \mathrm{MeV}$ protons. The beam energy spread is about $0.1 \%$ when the microprobe system is operated in the dispersive mode (see also Section 4.1), thus corresponding with a value of about $3 \mathrm{keV}$ for a $3 \mathrm{MeV}$ beam energy [MUT95]

The proton energy loss is subject to statistical fluctuations and hence protons impinging on the sample with identical initial energy $E_{p, 0}$ do not have exactly the same energy after passing through the sample. This statistical luctuation about the mean energy loss is called energy straggling. The energy distribution of the protonis that traversed the specimen is approximately Gaussian and can be estimated using Bohr straggling theory [CHU78]. For a biological sample with an areal density of $0.1 \mathrm{mg} / \mathrm{cm}^{2}$ Bohr straggling amounts to about $4 \mathrm{keV}$.

The total energy resolution FWHM (full width at half maximum), and thus the differences in thickness or areal mass density that can be resolved, is a convolution of the beam energy spread, the detector energy resolution and the width of the straggling distribution. Since the proton energy loss is the contrast parameter and not the number of detected protons, a twodimensional thickness distribution can be obtained when at least one proton per pixel in the scanned area is recorded. Inaccuracy of the determined areal mass density is governed solely by the energy measurement and not by counting statistics. However, by calculating the mean energy loss or the median value of a number of energy measurements at each position the accuracy of the energy loss measurement, und hence contrast, can be improved. Sealock et al. [SEA83] showed that the thickness resolution is inversely proportional to the square root of the number of protons detected. For a more comprehensive discussion on methods to efficiently reduce noise in STIM analysis the reader is referred to Section 4.2 .

\subsection{Determination of elemental concentration and limit of detection}

To be able to calculate an absolute value for the concentration of a trace element, first the solid angles of the X-ray detector and of the NBS and NFS detectors should be determined. In our case a reference standard, consisting of a polycarbonate backing with an evaporated metal layer (mostly nickel) is used for the external calibration of the X-ray detector and of both particle detectors. Since both the thickness and composition of the calibration sample are 
known, the solid angles can be deduced from measurement of characteristic X-rays and of scattered protons. To determine the solid angle of the X-ray detector Eq. 2.13 can be applied with Eq. 2.7, which can be written as:

$$
\frac{\Omega}{4 \pi}=\frac{Y\left(X_{c a l}\right) M_{c a l}}{(\rho t)_{c a l} N_{p, c a l} N_{a p} \sigma_{X_{c a l}}^{\text {prod }}\left(E_{p, 0}\right) \in\left(E_{X_{i a l}}\right) T_{a}\left(X_{c a l}\right)}
$$

where the subseript cal stands for the calibration conditions. From Eqs. 2.17 and 2.18 it follows that the solid angles for the NBS and NFS particle detectors are given by:

$$
\Omega_{N B S}=\frac{Y_{N B S}(c a l) M_{c a l}}{(\rho t)_{c a l} N_{p, c a l} N_{a v} \sigma_{c a l}^{N B S}\left(E_{p, 0}\right)}
$$

and

$$
\Omega_{N F s}=\frac{Y_{N F S}(c a l) M_{c a l}}{(\rho t)_{c a l} N_{p, c a l} N_{a v} \sigma_{c a t}^{N F S}\left(E_{p, 0}\right)}
$$

After the calibration experiment a (biological) sample of unknown thickness and elemental composition is analyzed. Substituting the expressions for the detector solid angles into Eqs. $2.13,2.17$ and 2.18 and combining these formulas with Eq. 2.19, the concentration of a trace element $Z$ now can be written as:

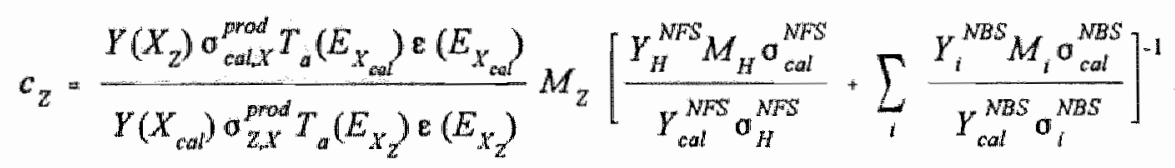

It is common practice to detect the characteristic $X$-rays and the scattered protons simultaneously during both the calibration experiments and the subsequent analysis of the biological specimen. As a result the number of protons $N_{p, c a l}$ impinging on the target during the calibration experiment and the number of protons $N_{p}$ hitting the biological specimen are cancelled out in this equation. Moreover, this expression enables one to calculate elemental concentrations without the necessity to know detector solid angles and the areal mass density of the calibration foil, since these quantities are eliminated from this equation. 
Equation 2.25 is only valid for thin samples. Otherwise, the determination of the areal mass density becomes more complex due to overlapping peaks in the NBS and NFS spectra. Besides, the attenuation of the characteristic X-rays in the sample and the energy (depth) dependence of the ionization cross section have to be taken into account. In this case a computer routine, developed by Jacobs [JAC94], is used to determine the local areal density from the NBS and NFS spectra. Subsequently the program PANEUT is used to calculate the elemental concentration corrected for thick-target effects using Eq. 2.12. The computer routines and the data base used for the calculations are discussed in Section 4.3 .

Now that the determination of the elemental concentration has been discussed, it is interesting to take a closer look at the minimum detectable concentration or limit of detection (LOD). The LOD for elements of interest in a particular matrix can be determined from the signal-to-background ratio in the PIXE spectrum. Generally, in X-ray microanalysis (both PIXE and EPMA) different criteria are used to define the limit of detection for an isolated peak in an X-ray spectrum. To provide the possibility to compare the LOD obtainable for different techniques (or different set-ups) it is therefore necessary to use the same criterion for the minimum detectable concentration. In this work, the following definition, recommended by the International Union of Pure and Applied Chemistry (IUPAC) is used:

$$
Y\left(X_{z}\right) \geq 3 \sqrt{N_{B}\left(E_{X_{Z}}\right)}
$$

In other words, the net content of an isolated peak in the X-ray spectrum should exceed 3 times the standard deviation in the background counts $N_{B}$ under this peak. For normally distributed peaks the factor 3 gives a confidence level of $99.86 \%$. In general the energy interval in which $N_{B}$ has to be determined equalls one, two or three times the FWHM of the Xray peak of interest. In this work the one FWHM option is adopted, since in some cases peaks that do not satisfy the two or three FWHM criterion still are visible in the X-ray spectrum.

The major sources of the continuous background in the PIXE spectrum are

i. projectile or primary bremsstrahlung

ii. secondary electron bremsstrahlung

iii. gamma radiation from nuclear reactions.

Projectile or primary bremsstrahlung results from the fact that a charged particle emits electromagnetic radiation when its speed or direction is changed. Therefore, the slowing down of projectiles in the specimen due to the Coulomb interaction with bound atomic electrons is accompanied by the emission of photons. The amount of energy lost and hence the energy of the emitted photons, ranges from zero up to the initial projectile energy. In classicall terms the intensity of the emitted projectile bremsstrablung is inversely proportional to the square of the projectile mass. For this reason the background is much more intense in case the sample is bombarded with electrons (EPMA) instead of the much heavier protons (PIXE). As a result a 100 times better sensitivity is claimed to be obtainable with PIXE compared to EPMA [JOH88]. 
With electron projectiles the primary bremsstrahung forms the major part of the continuous background under the characteristic peaks in the X-ray spectrum. For protons, however, projectile bremsstrahlung is predominated by secondary electron bremsstrahlung (SEB). SEB results from the deceleration of the electrons ejected from atoms in the jonization process, and its intensity falls off with increasing photon energy. In PIXE SEB is the major source of the continuous background.

The last major source of background in PIXE is gamma radiation resulting from nuclear reactions of the incoming protons with (trace)elements present in the sample or in parts of the microbeam set-up. These gamma rays can produce an intense and rather constant background due to Compton scattering in the detector. In calculations of the LOD the gamma rays emitted from target elements often are not included. However, for specific elements such as fluorine and sodium the gamma ray emission can contribute significantly to the total background in the $X$-ray spectrum [BOR80, MAU82], thereby worsening the detection limit for light elements.

Finally it should be noted that in practice the detection limits, which are often given for peaks that are isolated in the X-ray spectrum, can be much worse if the ability to resolve these peaks is seriously hampered by overlaps with other peaks. In X-ray microanalysis of biological tissue often the overlap of the $\mathrm{K}-\mathrm{K}_{\mathrm{p}}$ with the $\mathrm{Ca}-\mathrm{K}_{\mathrm{a}}$ is encountered. In order to verify both the influence of this peak overlap on the LOD and the stated difference in sensitivity between PIXE and EPMA in the practical situation, part of the next chapter is dedicated to the experimental determination of the minimum detectable calcium concentration in the presence of potassium by analysis of the same set of samples using both PIXE and EPMA.

Just like the ionization cross section contributes to the probability for the production of a characteristic $X$-ray, the probability for the production of bremsstrahlung photons in a distinct energy region in the $X$-ray spectrum is given by the differential cross section for the production of bremsstrahlung of element $j$. If both bremsstrahlung and gamma-ray background are taken into account the total background yield $N_{B}\left(E_{\mathcal{W}}\right)$ in the energy interval $E_{X} \pm 1 / 2 \Delta E_{d}$ is given by:

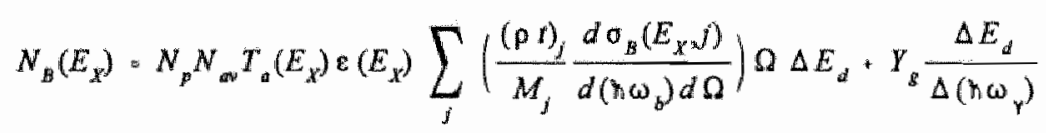

with

\begin{tabular}{|c|c|c|}
\hline$(\rho t)_{j}$ & $=$ & areal mass density of natrix element $j$ in the sample $\left(\mathrm{g} / \mathrm{cm}^{2}\right)$ \\
\hline$d a_{B}\left(E_{X} \cdot i\right)$ & \multirow{2}{*}{$=$} & \\
\hline$d(\operatorname{trcsin}) d \theta$ & & energy $h \omega_{b}$ of element $j\left(\mathrm{~cm}^{2} / \mathrm{keV} / \mathrm{sr}\right)$ \\
\hline$\Omega$ & $=$ & solid angle of X-ray detector (sr) \\
\hline$\Delta E_{d}$ & $=$ & $\begin{array}{l}\text { energy interval, often the energy resolution (FWHM) of the X-ray } \\
\text { detector (keV) }\end{array}$ \\
\hline$Y_{g} \Delta(n \omega)$ & $=$ & gamma-ray background yield at energy $\uparrow_{h} \omega_{\gamma}$ per energy interval $\left(\mathrm{keV}^{* 1}\right)$ \\
\hline
\end{tabular}


Note that the total bremsstrahlung background is obtained by summation over the contribution of all matrix elements $j$ present in the sample. A more detailed description of the background production in PIXE can be found in [ISH90] and [KRU92].

When the formulas for both the characteristic X-ray yield (Eq 2.13) and the total background yield (Eq. 2.27) are substituted in Eq. 2.26, a rather complex expression for the minimum detectable concentration is obtained. However, if the contribution of the gamma-ray background to the total background is neglected it follows that five different quantities that determine the limit of detection can be distinguished:

$$
L O D=12 \pi \sqrt{\frac{\Delta E_{d}}{N_{a v} N_{p} \Omega T_{a}\left(E_{X_{Z}}\right) \varepsilon\left(E_{X_{Z}}\right)}} \cdot \frac{\sqrt{\left(\frac{d \sigma_{B}}{d\left(\hbar \omega_{b}\right) d \Omega}\right)}}{\sigma_{Z_{n} Y}^{\text {prod }}}
$$

where $\Delta E_{d}$ represents the energy resolution of the $X$-ray detector.

The ratio between the cross sections for background praduction and the characteristic Xray production is determined by the choice of the projectile and its energy. As was discussed before the use of protons instead of electrons is preferred if low limits of detection are required. A beam energy of $2-4 \mathrm{MeV}$ is most frequently used in PIXE, since at lower proton energies the count rate is lowered due to a steep decrease in the ionization cross section, while at higher energies an increased probability of nuclear reactions, and thus a higher gamma-ray background, is observed.

Over the last years the energy resolution of commercially available energy dispersive X-ray detectors has not improved significantly. However, both the use of large area detectors (with an active area of $60-80 \mathrm{~mm}^{2}$ instead of $30 \mathrm{~mm}^{2}$ ) and the employment of a smart detectortarget geometry [WAT95] result in a larger solid angle of detection $\Omega$, thereby improving the LOD. The total number of protons impinging on the specimen $N_{\mu}$ simply is the product of the beam current and the total measurement time, divided by the proton charge. This means that the LOD can be improved by either using a higher beam current or by increasing the measurement time. In a proton microprobe, however, an increase in beam current often can only be obtained at the cost of a larger beam spot size and thus a reduction in spatial resolution. In addition, the use of increased beam currents might lead to serious specimen damage. Therefore the most obvious way to improve the LOD is longer irradiation of the sample. In this case the duration of the measurement will be a compromise between the desired sensitivity and the cost and availability of the used microprobe set-up. Moreover, the mechanical stability of the instrumental set-up should be considered.

From Eq. 2.28 it follows that the limit of detection is inversely proportional to the square root of the total collected charge $Q$. For this reason it is interesting to estimate the obtainable LOD in $\mu$-PIXE compared to standard broad beam PIXE, since the beam current is approximately two orders of magnitude less for the microbeam. Moreover, if a scanning 
proton beam is employed the total deposited charge is divided over all the positions in the scan pattern (pixels). As result the lowest detectable concentration of an element for one pixel becomes worse if the number of pixels is increased while keeping the measurement time constant.

Figure 2.7 shows the calculated detection limit for $\mathrm{Na}, \mathrm{Mg}, \mathrm{K}$ and $\mathrm{Ca}$ as a function of the total measurement time per pixel in case a beam current of $100 \mathrm{pA}$ is employed (solid line). The values for the LOD for short measurement times have been extrapolated from measurements and callculations of the LOD for a total accumulated charge of $100 \mu \mathrm{C}$ [JOH88]. Since the detection limit does not vary drastically for $11<Z<22$, approximately the same value for the LOD for $\mathrm{Na}_{3} \mathrm{Mg}, \mathrm{K}$ and $\mathrm{Ca}$ is found.

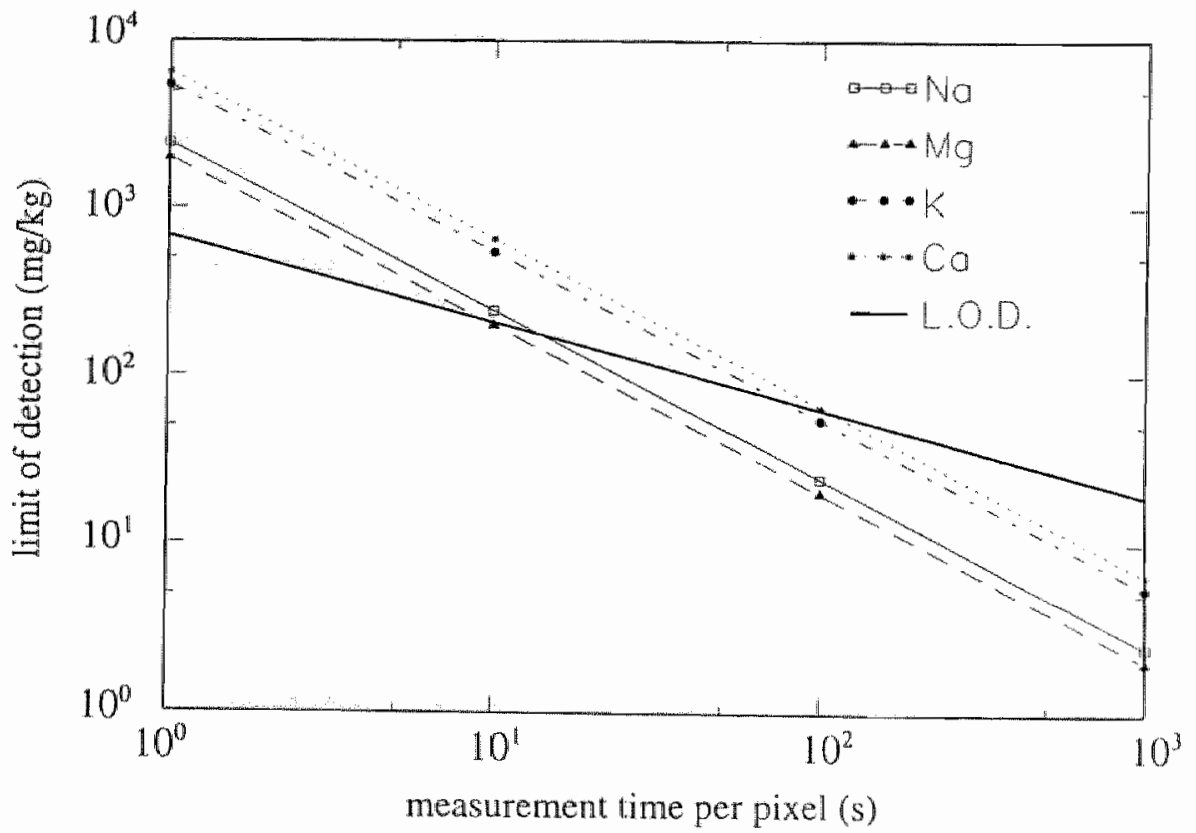

Figure 2.7: Limit of detection (LOD) for several elements as a function of the meantement ime. per pixel. The solid line shows the LOD, calculated for a beam current of $100 \mathrm{pA}$. The fow parallel lines give the concentration of $\mathrm{Na} . \mathrm{Mg}$. Kand Ca for which the statistical error in the mumber of coums in the peak $N / N=0.20$. with $N$ the number of conmts in the peak.

The calculation of the LOD as a function of the total time each pixel has been irradiated provides the possibility to predict the obtainable sensitivity for a given scan size and total measurement time. However, in this calculation the absolute number of counts in the peak is not taken into account. The four parallel lines in Figure 2.7 show the concentration for $\mathrm{Na}$, 
$\mathrm{Mg}, \mathrm{K}$ and $\mathrm{Ca}$ for which the number of counts in the peak has a $20 \%$ confidence level, whereas the solid line shows the LOD for these elements. It is clear that for low measurement times per pixel, i.e., left of the intersection of the solid line and the four dotted lines, the LOD is determined by the count rate, while for longer measurement times the detection limit is set by the criterium that the peak should exceed 3 times the statistical uncertainty in the background under the peak of interest.

\subsection{Summary and concluding remarks}

In this chapter the basic theory of the three most commonly used techniques in proton microprobe analysis, i.e., PIXE, RBS and STIM, is presented. The calculation of the elemental concentration and the lowest detectable mass fraction obtainable with a combination of these techniques are also discussed in this chapter. It was shown that even in biological samples with a thickness of several $\mu \mathrm{m}$ for a selected number of elements of interest, such as $\mathrm{Na}$ and $\mathrm{Mg}$, thick-target effects have to be taken into account. Calculations indicated that for samples with an areal mass density of $0.6 \mathrm{mg} / \mathrm{cm}^{2}$ neglecting thick target effects would even result in an underestimation of the $\mathrm{Na}$ concentration by a factor of 2 . An experimental validation of these calculations was obtained by proton microprobe anallysis of samples with a known and homogeneous concentration distribution of $\mathrm{Na}$ and $\mathrm{K}$. In addition it was shown that the matrix composition of the sample has a minor influence on the elemental concentrations as determined from proton microprobe experiments. As a result in most cases it is sufficient to determine the (local) thickness of the specimen and to use a mean matrix composition for the calculation of the concentration of trace elements present in the sample. 
Chapler 2 


\section{Chapter 3}

\section{Practical comparison between PIXE and EPMA}

\subsection{Introduction}

Since the electron microprobe is widely used and plays an important role in analyses in the biomedical field, it is of interest to compare the potentials of micro-PIXE with EPMA. In comparison to the electron microprobe, the proton microprobe has an inferior spatial resolution, but overall a much better sensitivity. As was discussed in Section 2.3, for protons the bremsstrahlung production is much lower as compared to bremsstrahlung generated by electrons, resulting in detection limits in the $\mathrm{mg} \mathrm{kg}^{-1}$ or parts per million $(\mathrm{ppm}$ ) range. For conventional energy dispersive $X$-ray microanalysis using electrons the detection limits are roughly between 200 and 1000 ppm.

In the case of overlapping peaks in the $\mathrm{X}$-ray spectrum, such as the overlap of the $\mathrm{K}-\mathrm{K}_{\beta}$ and Ca-K $\mathrm{K}_{\mathrm{a}}$ peaks, quantitative analysis of elements can be seriously hampered, particularly in the case of low elemental content. Due to the fact that in most biological specimens the Ca-K peak is relatively weak compared with the $K-K_{\beta}$ peak it will appear as a shoulder on the $K-K_{\beta}$ peak, making accurate quantitative analysis of low $\mathrm{Ca}$ in the presence of high $\mathrm{K}$ levels difficult.

The work presented in this chapter is confined to the experimental determination of the minimum detectable $\mathrm{Ca}$ content by analysis of the same set of standards ${ }^{1}$ in biologically relevant matrices using both proton and electron induced X-ray microanalysis. Three different sets of matrices were used and evaluated for their applicability, viz sucrose, bovine serum albumin (BSA) and polyethylene glycol (PEG) containing known concentrations of $\mathrm{CaCl}_{2}$. To determine to what extent the determination of $\mathrm{Ca}$ is hampered by the presence of high $\mathrm{K}$ levels, two other series also containing high $\mathrm{K}$ levels were analyzed. The materials and methods described in this section, such as the sample preparation methods, as well as the description of the proton microprobe and the electron microscope, are discussed in more detail in Chapter 4 and Chapter 5.

\subsection{Preparation of calibration samples}

For the calibration of measurements of cryosectioned biological specimens, cryosectioned specimens as standards are advisable, because of their resemblance with biological tissue. In this study both bovine serum albumin (BSA) and polyethylene glycol (PEG) were used to prepare cryosectioned material of known composition ("standards"). These materials can be sectioned to

"In this chapter the word "standard" or "calibration sample" is used for specimens containing known anounts of naterial. It should be noled, howewer, that in principle for PXXE use of these standards is not required to obtain absolute values far the contem of each element present in a (biological) specimen in case a Ni calibration foil of known thickness is used to determine the detechor solid angles (see Chapter 2). 
a thickness comparable to the thickness of cryosections used for analysis of biological tissue. Since the detection limit depends on the specimen thickness, these standards give a direct measure of the limits of detection attainable in biological tissue.

Kitazawa and coworkers [KIT83] have shown that good results can be obtained with BSA standards for use in the electron microscope. According to Wolosewick [WOL84] PEG can be used as an embedding medium and is easy to section in microtomes. In addition, our own experiments indicated that PIXE analysis of pure PEG blocks revealed minor presence of contaminating elements. Both properties make PEG a suitable matrix for the preparation of cryosectioned standards. As an alternative to cryosectioned standards the use of standard specimens consisting of sucrose spray droplets has been proposed by Linders [LIN84], since no time consuming procedures like sectioning and freeze-drying are required to prepare these standards.

In this section the preparation of these standards for PIXE analysis will be described. In the preparation an attempt was made to mimic the preparation of biological material for PIXE as much as possible. Also the composition of the material was chosen such that PIXE analysis would provide relevant information for the PIXE analysis of biological material. The PIXE analysis was carried out on frozen, sectioned and freeze-dried solutions of organic material supplemented with organic salts. From the use of standards ("model systems") for PIXE analysis the merits of these standards were evaluated as well as the relevance of these collected data for the analysis of biological material.

\section{Preparation of sucrose standards}

A stock solution with $5 \%(w / v)$ sucrose in water was prepared. To prevent microbial contamination $1 \mathrm{mM}$ sodiumazide $\left(\mathrm{NaN}_{3}\right)$ was added to the stock solution. Calcium standards were prepared by dissolving a known amount of $\mathrm{CaCl}_{2}, 2 \mathrm{H}_{2} \mathrm{O}$ (from Merck, Darmstadt, Germany) in an aliquot of this stock solution. Subsequently, this solution was diluted several times with the sucrose stock solution to obtain calibration series with $\mathrm{Ca}$ concentrations varying from $10 \mu \mathrm{M}$ to $100 \mathrm{mM}$. In addition to a Ca calibration series without potassium, two series containing $70 \mathrm{mM}$ and $140 \mathrm{mMK}$, respectively, were prepared using $\mathrm{KCl}$ (from Merck, Darmstadt, Germany)

For use in the electron microscope the standard solutions were nebulized over thin $(50-100 \mathrm{~nm})$ Formvar films mounted on 200 mesh $\mathrm{Cu}$ grids. The microdroplets obtained in this way had a diameter of 1 to $2 \mu \mathrm{m}$. The specimens were allowed to dry in air and coated with a conducting carbon layer prior to analysis.

Identical stock solutions were used for the preparation of calibration series for use in the proton microprobe. For imaging purposes the specimen chamber of the proton microprobe set-up is equipped with a light microscope (see also Section 4.1). However, due to the long working distance of the microscope (low resolving power), it is difficult to visualize the microdroplets as used for electron microscopy. Therefore larger droplets, with a diameter ranging from about 10 $500 \mu \mathrm{m}$, were prepared for use in the proton microprobe using a conventional air-brush to spray the standard solutions. The droplets were caught on a Formvar foil (thickness $100-200 \mathrm{~nm}$ ) covering a $10 \mathrm{~mm}$ diameter hole in a plastic sheet. This sheet can be clasped in a regular slide mount which fits into the target wheel inside the vacuum chamber. 


\section{Preparation of bovine serwm albumin (BSA) standards}

A stock solution with $20 \%$ (w/v) BSA (from Sigma, St. Louis, USA, No. A-4503) was prepared. Standards were made by adding known amounts of $\mathrm{CaCl}_{2} .2 \mathrm{H}_{2} \mathrm{O}$ and $\mathrm{KCl}$ to this stock solution. Subsequently this solution was diluted several times, so that a series with a known protein/salt ratio was obtained. The added amounts of mineral salts were chosen so, that the $\mathrm{Ca}$ mass fraction varied from $10 \mathrm{ppm}$ to $10,000 \mathrm{ppm}$ dry weight for three different calibration series containing no $\mathrm{K}, 5000 \mathrm{ppm} \mathrm{K}$ and $10,000 \mathrm{ppm} \mathrm{K}$ dry weight, respectively. The BSA-mineral salt mixture was frozen in melting isopentane and the frozen sections were sectioned at $-20^{\circ} \mathrm{C}$ in a cryostat microtome. The sections were cut at a thickness of about $15 \mu \mathrm{m}$ and were collected on Formvar backing foils. Subsequently these sections were freeze-dried prior to analysis with the proton microprobe. BSA was not used for the preparation of sections for the purpose of electron microscope analysis. Since the BSA used for the preparation of the standards was found to be contaminated with $\mathrm{Ca}$, a second calibration series was prepared using the BSA after overnight dialysis against $0.1 \mathrm{mM}$ ammonium bicarbonate buffer $(\mathrm{pH}=8.0)$. The dialysed $\mathrm{BSA}$ solution was subsequently freeze-dried.

\section{Preparation of polyethylene glycol (PEG) standards}

In this case the stock solution consisted of a $20 \%(\mathrm{w} / \mathrm{v})$ PEG (M.W. 18500, from Polyscience No. 4686) solution. To make a calcium calibration series a known amount of $\mathrm{CaCl}_{2} \cdot 2 \mathrm{H}_{2} \mathrm{O}$ was added to an aliquot of the stock solution. This solution was diluted several times with the stock solution to obtain calibration series with $\mathrm{Ca}$ contents ranging from 10 to $10,000 \mathrm{ppm} \mathrm{Ca}$ dry weight. Two other calcium series were prepared which contained 5000 and $10,000 \mathrm{ppm} \mathrm{K}$ dry weight, respectively. The PEG-salt mixture was frozen in melting isopentane. For use in the proton microprobe, sections were cut at $-20^{\circ} \mathrm{C}$ in a cryostat microtome at a thickness of about $15 \mu \mathrm{m}$. For electron microscopy, thinner (about 100-200 nm) sections were cut in a cryomicrotome (from Cryonova LKB, Stockholm, Sweden) at about $-90^{\circ} \mathrm{C}$. Both proton and electron probe sections were collected on thin Formvar films (see above).

\subsection{Electron and proton probe $\mathrm{X}$-ray microanalysis}

\section{Electron probe analysis}

Electron probe analysis was carried out with an EDAX PV9900 X-ray microanalysis system with a $30 \mathrm{~mm}^{2} \mathrm{Si}(\mathrm{Li})$ energy-dispersive detector mounted on a Philips CM-12 electron microscope. The microscope, which is equipped with a $\mathrm{LaB}_{6}$ electron gun, was operated in the scanning transmission mode at $120 \mathrm{kV}$. The specimens were kept at ambient temperature during analysis.

The analysis of X-ray spectra obtained from electron microscopy was performed using the EDAX PBTHIN-routine, a program based on Roomans' paper [ROO88] and specially designed for the analysis of thin-section biological specimens. An important feature of this program is the use of the peak-to-background intensity ratio ( $\mathrm{P} / \mathrm{B}$-ratio), including corrections for the fact that the observed continuum intensity also contains an extraneous component resulting from interaction of electrons and of $X$-ray scattering outside the analyzed volume.

Sucrose microdroplets with a diameter in the order of $2 \mu \mathrm{m}$ were irradiated by a scanining 
electron beam using a scanining area enclosing the specimen. The beam current (monitored by the indication on the display of the filament current) was kept constant and usually in the order of 0.2 $0.3 \mathrm{nA}$.

PEG standards were analyzed using a scaming beam with a beam current comparable to the current used for the analysis of the sucrose microdroplets. In contrast with the measurement of the sucrose standards the scanning area was completely enveloped by the specimen. For all specimens analysis times of about 2000 s were used.

\section{Proton microprobe analysis}

To prevent backscattered protons from entering the $\mathrm{X}$-ray detector, thereby distorting the detector signal, a $15.5 \mathrm{mg} \cdot \mathrm{cm}^{-2}$ Be absorber was placed in front of the Si(Li)-detector during all proton microprobe experiments. The measured $\mathrm{X}$-ray yields were corrected for the absorption of $X$-rays in this absorber. The solid angles of both the $\mathrm{X}$-ray detector and the two particle detectors were determined using a reference standard. This standard consists of a polycarbonate foil with an evaporated nickel layer with a mass thickness of $7.0 \mu \mathrm{g} \cdot \mathrm{cm}^{-2}$. This value was determined by the content of the Ni-K er peak in the PIXE spectrum and from the width of the Ni peak in the contesponding RBS spectrum resulting from irradiation with $3 \mathrm{MeV}$ protons. Both values agree within 5\% [MUN94]. The cross sections for the elastic scattering of protons (in the nonRutherford region) were taken from the work published by Amirikas and coworkers [AMI93] All other data needed for the calculation of the calcium content of the standards were taken from literature except for the data on the detector efficiency of the X-ray detector, which has been determined experimentally [MUN94].

Analysis of the X-ray spectra has been performed using the AXIL software [MAE86] Although normally used for the analysis of proton induced X-ray spectra, AXIL is also suited for the evaluation of spectra generated during electron irradiation. It should be kept in mind, that the AXTL program offers no possibility to make corrections of the continuum intensity for extraneous contributions. Electron induced X-ray spectra cam be analyzed using both the PBTHIN program and the AXIL routine. This offers the possibility to compare the procedures to obtain the net peak content of the characteristic peaks present in the $\mathrm{X}$-ray spectra applied by the two programs. Furthermore this enables one to verify the specific influence of the correction of the continuum intensity made by the PBTHIN program.

The air-dried sucrose droplets were analyzed using a scanning proton beam of about $3 \times 3 \mu \mathrm{m}^{2}$ with a bean current of about $100 \mathrm{pA}$. To determine the mean content of the dissolved elements the scanning area enveloped the whole droplet.

Both BSA and PEG standards were analyzed using a scanning proton beam with a scan frequency of $5 \mathrm{kHz}$. The beam had a spot size of about $10 \times 10 \mathrm{~mm}^{2}$ and was scanned across the cryosections covering a large area (about $150 \times 150 \mu \mathrm{m}^{2}$ ). This enables one to check whether the dissolved mineral salts are distributed uniformly. If the sample shows acceptable homogeneity the total X-ray spectrim, made up by the sum of the individual spectra measured at every pixel of the scan pattern, can be used to calculate the mean $C a$ and $K$ content. Due to the larger beam spot size a beam current of about $250 \mathrm{pA}$ could be used. As a result the total accumulated charge was about $200-300 \mathrm{nC}$ during an analysis time of about $1000 \mathrm{~s}$. 


\subsection{Results and discussion}

\section{Sucrose standards}

Figure 3.1 shows the result of the electron probe analysis of the sucrose microdroplets, using the AXIL routine to fit the X-ray spectra. The known $\mathrm{Ca}$ contents are displayed on the horizontal axis, whereas the peak-to-background ratio (P/B-ratio) is plotted on the vertical axis. The continuum intensity, with the continuum region chosen between 4.5 and $6.0 \mathrm{keV}$, was corrected for the difference in the weighted sum of $Z^{2} / A$ for all elements present in the analyzed volume of the specimen [ROO88].

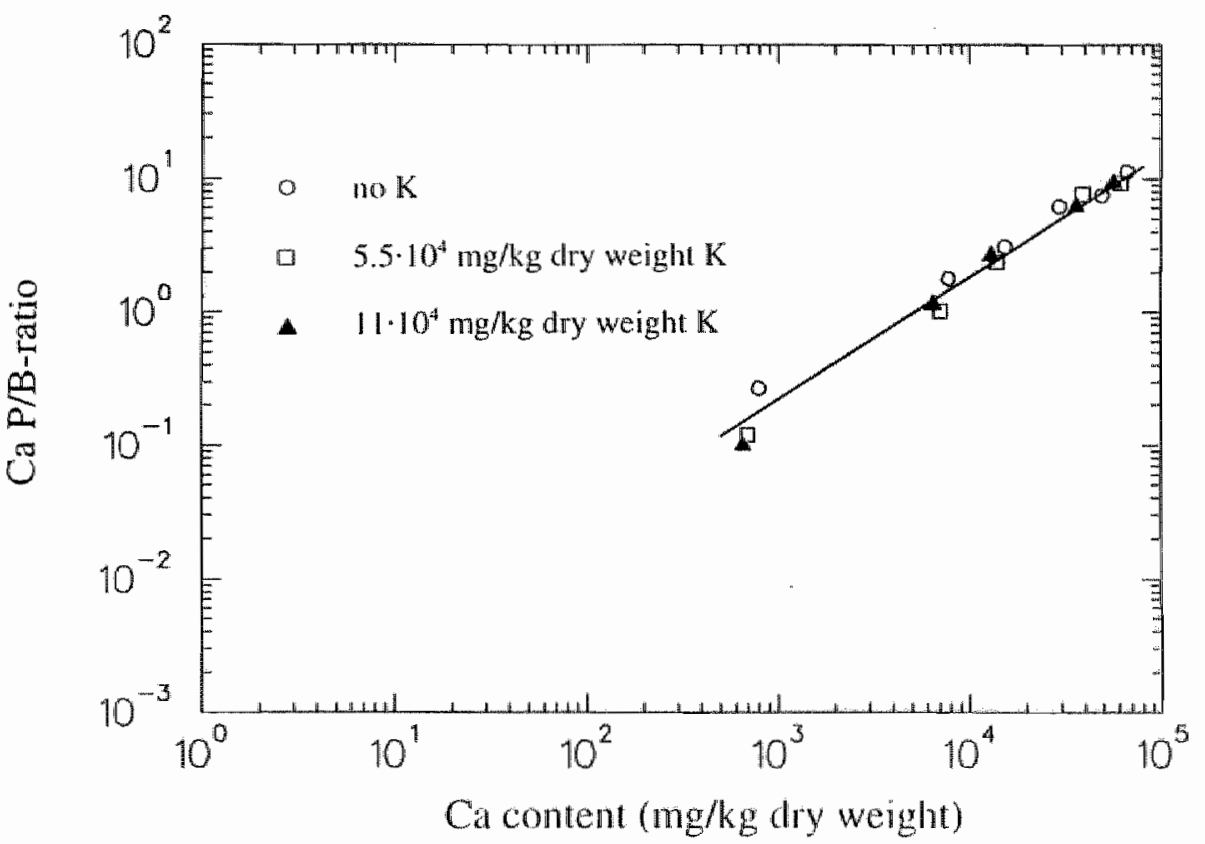

Figure 3.1: The Ca P/B-ratio as a finction of the calciun content of a series of sucrose microdroplets deternined with electron probe analysis. The beam current was 0.2-0.3 $\mathrm{mA}$, the analysis time about $2000 \mathrm{~s}$ and the accele ration voltage $120 \mathrm{~kW}$.

Analysis of droplets containing to $800 \mathrm{mg} / \mathrm{kg} \mathrm{Ca}$ dry weight $(800 \mathrm{ppm})$ showed a Ca peak that still exceeds the statistical uncertainty in the background (see Fig. 3.1). When a 10 times lower $\mathrm{Ca}$ content was analyzed no significant $\mathrm{Ca}$ signal could be detected. This implies that the minimum detectable mass fraction for $\mathrm{Ca}$ using our electron microprobe system lies in the range 80 $800 \mathrm{ppm}$ Ca under the conditions used for analysis. 
It can be seen from Figure 3.1 that the determination of calcium present in the sucrose standards is not influenced by the presence of potassium in the sucrose matrix down to the lowest detected $\mathrm{Ca}$ content $(800 \mathrm{ppm})$. This means that for the contents of $\mathrm{Ca}$ and $\mathrm{K}$ investigated the $\mathrm{K}$ $\mathrm{K}_{\beta} \mathrm{Ca}-\mathrm{K}_{w}$ overlap is treated correctly by the AXIL program down to a Ca/K"ratio of about 0.01 .

When the same set of data was analyzed with the PBTHIN-routine, in contrast to the analysis with AXIL no linear relation between the dissolved amount of calcium and the P/B-ratio was found. This can be explained as follows. Due to the correction for the exiraneous background by the PBTHIN-jrogram, the corrected continuum intensity is lower than the uncorrected background. When the ratio of the corrected and the uncorrected background is very low $(<0.10$ in our case), it can be concluded that the analyzed specimen is very thin. This results in a strong contribution of the thickness of the Formvar foil to the calculated P/B-ratio. For this reason it is advised not to use the PBTHIN-routine when this ratio gets below $40 \%$ to avoid errors (as also stated in the PBTHIN user manual).

Proton probe analysis of the sucrose microdroplets showed that the use of these droplets is unreliable. It appeared that droplets sprayed from the same stock solution showed large variations in calcium content, specially for droplets of varying diameter (data not shown). A possible explanation for this effect is that electrostatic charges induced at the spray nozzle result in an uneven distribution of ions over larger and smaller droplets. Moreover, RBS analysis of the specimens showed that the mass thickness of the sucrose standards ranged from 0.02 to $0.1 \mathrm{mg} / \mathrm{cm}^{2}$. As a result, the contribution of the mass thickness of the Formvar backing (about $\left.0.01 \mathrm{mg} / \mathrm{cm}^{2}\right)$ to the calculated mass thickness of the specimen, used to normalize the PIXE results, can not be neglected.

The use of sucrose droplets as matrices for the analysis of ions in electron microscopy has been reported before [LIN82, LIN84]. The present results indicate that for electron microscopy the dissolved and measured calcium contents are linearly related if droplets of roughly the same diameter were analyzed. However, no reliable results could be obtained when droplets of various dimensions were used in the proton microprobe. Moreover, analysis of the sucrose microdroplets showed that correction for extraneous background production in quantitative electron microscopy is only allowed if the specimens are not too thin (i.e., if the continuum background corrected for extraneous contributions does not drop below $40 \%$ of the uncorrected continuum). The low dry mass of the droplets compared to the Formvar support film also imposes problems when an accurate determination of the sample mass thickness using RBS is required. Moreover the sucrose droplets hardly resemble with cryosectioned biological tissue, i.e., the ratio between inorganic matter and organic matter is much higher in the sucrose model systems used than in biological material. Further adjustments to this model system by using high sucrose concentrations (mimicking the organic content of biological material) result in high viscosity solutions that are impossible to spray with the equipment described. For this reason it can be concluded that it is not advisable to use these standards for making $\mathrm{Ca}$ standards for both PIXE and EPMA.

\section{BSA standards}

Proton probe analysis of non-dialysed BSA standards (without the addition of salts) showed a high $\mathrm{Ca}$ level ( $>1000 \mathrm{ppm}$ dry weight, data not shown). For this reason dialysed BSA was 
subsequently used for the preparation of standards. The results of proton microprobe analysis of the dialysed BSA standlards are displayed in Figure 3.2. It can be seen that the dissolved and the measured Ca content are linearly related down to a calcium level of $100 \mathrm{ppm}$ dry weight. However, using the criterium $Y_{2} \geq 3 \sqrt{ } N_{B}$, from the detection of the sample containing $100 \mathrm{ppm} \mathrm{Ca}$ (total accumulated charge $\mathrm{Q}=275 \mathrm{nC}$ ) a minimum detectable content of $6 \mathrm{ppm} \mathrm{Ca}$ was calculated for the same analysis conditions.

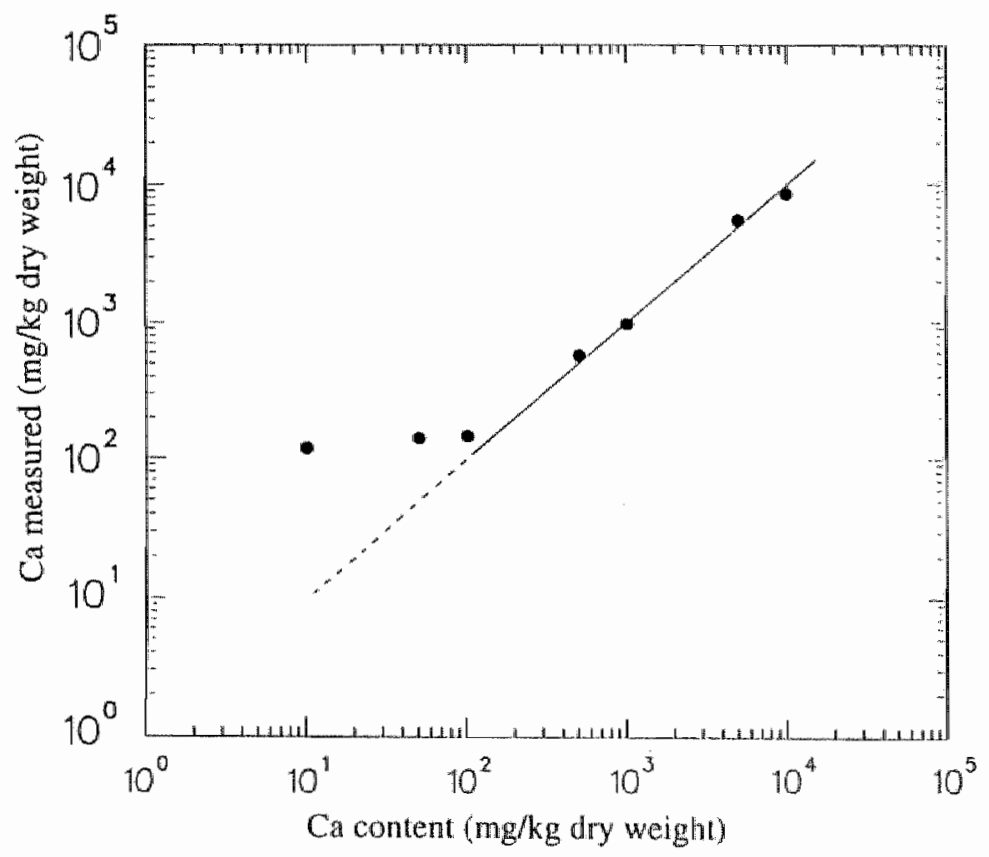

Figure 3.2: $\quad$ PIXE amalysis of Ca standards in a diatysed matrix of bovine serum albumin (BSA). The $B S A$ was dialysed overnight against an $0.1 \mathrm{M}$ ammonium bicarbonate buffer $(\mathrm{pH}=8.0$ ) and was freeze-dried subsequently. $\mathrm{A} 3 \mathrm{MeV}$ proton beam was used with a beam current of about $0.25 \mathrm{nA}$. The total accumstated charge $Q$ amounied to about $250 \mathrm{nC}$ for all specimens.

Dialysis of BSA resulted in a substantial reduction of the $\mathrm{Ca}$ level, i.e., from more than 1000 down to $100 \mathrm{ppm} \mathrm{Ca}$. However, taking into account the sensitivity of the PIXE technique, the reduction appeared not to be enough. Therefore this BSA matrix is not suitable for making standards with low Ca content.

\section{PEG standards}

From the result of the electron probe analysis of the PEG standards (Figure 3.3) it was determined that the detection limit for $\mathrm{Ca}$ lies between 500 and $1000 \mathrm{ppm}$, either in the presence or absence 
of $K$. For the PIXE analysis of Ca in a PEG matrix the PEG samples were analyzed using a scanning proton beam, covering an area of about 150 is $150 \mathrm{~m}^{2}$. Whin whe statistical uncertainty all analyzed PEG samples showed a homogeneous Ca distribution. Therefore the total X-ray spectrum was used to calculate the mean Ca content of the scanned area. The results are displayed in Figure 3.4. It is cleat from this figure that for standards which are free of potassum a good linear relation between the known and measured Ca contents clown to 10 ppn of Ca is found. Aso when 5,000 or 10,000 ppm $K$ has been added to the PEG standards it is still posstble to detect 10 ppm of Ca.

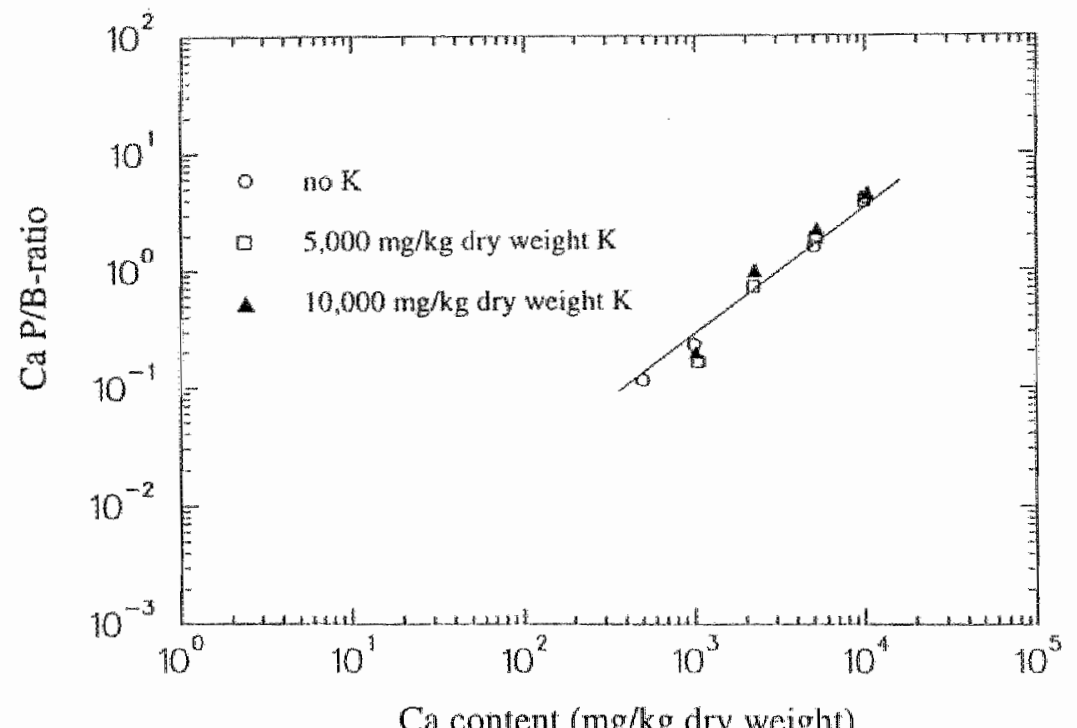

Figure 3.3: EPMA analysis of Ca standards in a polyethylene glycol (PEG) matrix. Analysis conditions: $120 \mathrm{kV}$ acceleration wolrage, foral accumulated charge $\mathrm{Q}=200-400 \mathrm{nC}$.

For the lowest $\mathrm{Ca}$ contents the deviation from the straight line can be caused by the $\mathrm{Ca}$ contamination level of the sample and/or the uncertainty in the fitted peak-area due to bad statistics. For the PEG samples containing 5000 and $10,000 \mathrm{ppm} \mathrm{K}$ this deviation can originate from the $\mathrm{K}-\mathrm{K}_{\mathrm{a}} / \mathrm{Ca}-\mathrm{K}_{\text {of }}$ peaks overlap as well. Analysis of samples to which $\mathrm{Ca}$ was added at a level of 1 ppm showed a measured $\mathrm{Ca}$ content of about $15 \mathrm{ppm} \mathrm{Ca}$ dry weight, indicating a blank value in the same order. "Therefore it can be concluded that a possible influence of high $K$ levels on the determination of low $\mathrm{Ca}$ contents is obscured by the contamination level of the PEG specimens. However, the PIXE results show that it is possible to detect $10 \mathrm{ppm} \mathrm{Ca}$ in the presence of 10,000 ppm $\mathrm{K}$, corresponding to a $\mathrm{Ca} / \mathrm{K}$-ratio of 0.001 . 


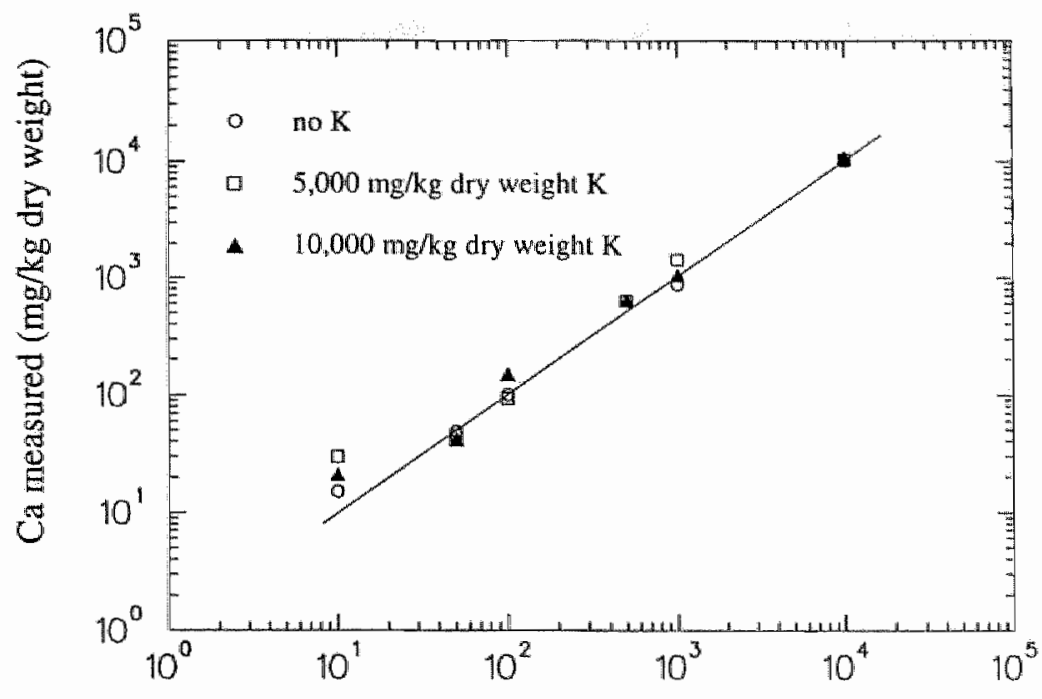

Ca content (mg/kg dry weight)

Figure 3.4: PLXE analysis of Ca standards in a polyethylene glycol (PEG) matrix. The specimens were irradiated with a $3 \mathrm{MeV}$ proton beam up to $Q=200.300 \mathrm{nC}$.

\subsection{Summary and concluding remarks}

The use of sucrose droplets as $\mathrm{Ca}$ standards is not recommended because of $i$ ) the dependence of the content on the size of the droplets, ii) the limitation to thin samples and iii) the low resemblance to cryosectioned biological tissue.

In this study both BSA and PEG were used to produce cryosectioned standards. To make standards with a low Ca content it is necessary to use a matrix with a low contamination level. Proton probe analysis of the BSA standards showed that the dissolved and measured Ca contents were linearly related down to $100 \mathrm{ppm}$ Ca dry weight. However, a minimum detectable content of 6 ppm Ca was calculated from the peak to background ratio of BSA samples containing 100 ppm Ca. It appeared that the calcium content in dialysed BSA was still too high to apply the BSA matrix for the production of low Ca standards to be used in the proton microprobe. For this reason no parallel determinations were made using BSA to produce standards for electron microscopy. The best results were obtained using PEG as a matrix material. This material was already known for its good sectioning properties. However, to the best of our knowledge up to now no reference was made of the use of PEG for the production of cryosectioned standards for quantitative electron and proton induced $X$-ray microanalysis. $P E G$ is very suitable for the production of $\mathrm{Ca}$ standards in the low content range for both proton and electron induced $X$-ray 
microanalysis because of the low contamination level. Moreover, mapping of $\mathrm{Ca}$ showed a homogeneous distribution of $\mathrm{Ca}$ over the area that was scanned by the proton microbeam.

The present results obtained with PEG matrices indicate that the minimum detectable mass fraction for calcium is about two orders of magnitude better for PIXE than for EPMA when an appropriate matrix is used. It should be noted, however, that this result is strongly dependent on the specific properties of the used microprobe systems and the applied charge.

Theoretically it is difficult to predict the influence of high $\mathrm{K}$ levels on the detection of low $\mathrm{Ca}$ concentrations. For this reason it was determined experimentally. No significant influence of high $\mathrm{K}$ levels (up to 10,000 ppm) on the accurate detection of $\mathrm{Ca}$ with EPMA was found for $\mathrm{Ca}$ contents down to about $1000 \mathrm{ppm}$. For PIXE the same result was obtained for $\mathrm{Ca}$ contents down to $10 \mathrm{ppm}$. For lower Ca contents the effect of the $\mathrm{K}-\mathrm{K}_{\beta} / \mathrm{Ca}-\mathrm{K}_{\mathrm{a}}$ overlap is obscured by the $\mathrm{Ca}$ contamination level of the PEG samples.

It should be realized that the intracellular levels of $\mathrm{Ca}$ are much lower than those of $\mathrm{K}$. For instance, for normal heart tissue we found $\mathrm{Ca}$ and $\mathrm{K}$ levels inside cardiomyocytes in the order of 300 and $15,000 \mathrm{ppm}$ dry weight, respectively. For injured heart tissue $\mathrm{Ca}$ and $\mathrm{K}$ contents were in the order of $500-3,000 \mathrm{ppm}$ and $6,000 \mathrm{ppm}$ dry weight, respectively [VER95]. The Ca/K-ratio amounts to $\sim 0.02$ for normal tissue and to $\sim 0.25$ for injured tissue. This study indicates that the present PIXE procedures are sensitive enough to determine low $\mathrm{Ca}$ levels in the presence of much higher $\mathrm{K}$ levels.

In conclusion it can be stated that the data obtained with the standards or "model systems" have provided: i) a verification of the theoretical predictions on the analysis results and the procedures for the deconvolution of analytical data to 'real' concentrations in the starting solutions and ii) an answer to the question which instrumental set-up is to be used for the detection of elements in biological material. 


\section{Chapter 4}

\section{Technical aspects of proton microprobe analysis of biological material}

\subsection{Introduction}

In this chapter some specific experimental aspects of the analysis of biological tissue using a proton microprobe are discussed. The proton microprobe set-up of the Eindhoven University of Technology (EUT) is treated in Section 4.1. In order to be able to perform the analysis of biological tissue sections on the cellular level this set-up has been improved during the last years. An extensive description of the design and construction of the modernized proton microprobe set-up can be found in [MUT95]. In Section 4.1 only those aspects of the microbeam set-up are described that are necessary for understanding the experiments performed in this study. In addition various improvements that were introduced during this study and the calibration of two X-ray detectors will be discussed.

In case elemental concentrations are to be measured on the cellular level different cells are to be recognized. Moreover, the ability to visualize individual cells makes it possible to analyse the concentration of elements in these cells and to perform a cell-to-cell comparison within the same cryosection. In Section 4.2 four different techniques are described that can be applied for imaging purposes.

Section 4.3 is completely dedicated to the data acquisition, monitoring of data and data analysis. In this section the computer programs that are used to calculate the elemental concentration from the combined PIXE and NBS/NFS measurements are treated. Finally, the possible applications of the proton microprobe for quantitative analysis of biological material are summarized in Section 4.4 .

\subsection{Experimental set-up}

At the KernFysische Technieken (KFT) group of the physics department at the EUT an AVF (Azimuthal Varying Field) cyclotron (Philips, Eindhoven, the Netherlands) is used to accelerate protons, deuterons, ${ }^{3} \mathrm{He}$ and alpha-particles. The particles can be accelerated to an energy in the range of about 2 to $30 \mathrm{MeV}$. Johansson [JOH92] showed that a lower limit of detection can be realized in case $\alpha$-particles are used instead of protons. However, due to practical limitations of the Eindhoven cyclotron it is very difficult to produce an $\alpha$-particle microbeam with sufficiently high beam current. Moreover, biological material is especially sensitive to beam induced sample damage in case $\alpha$-particles are used. For this reason a proton beam with an energy of about $3 \mathrm{MeV}$ is used during all experiments described in the present study. For lower proton energy the ionization cross sections of most elements decrease (except for elements with $Z \leq 11$ ), whereas for higher energy the background from proton induced nuclear reactions rapidly increases. 


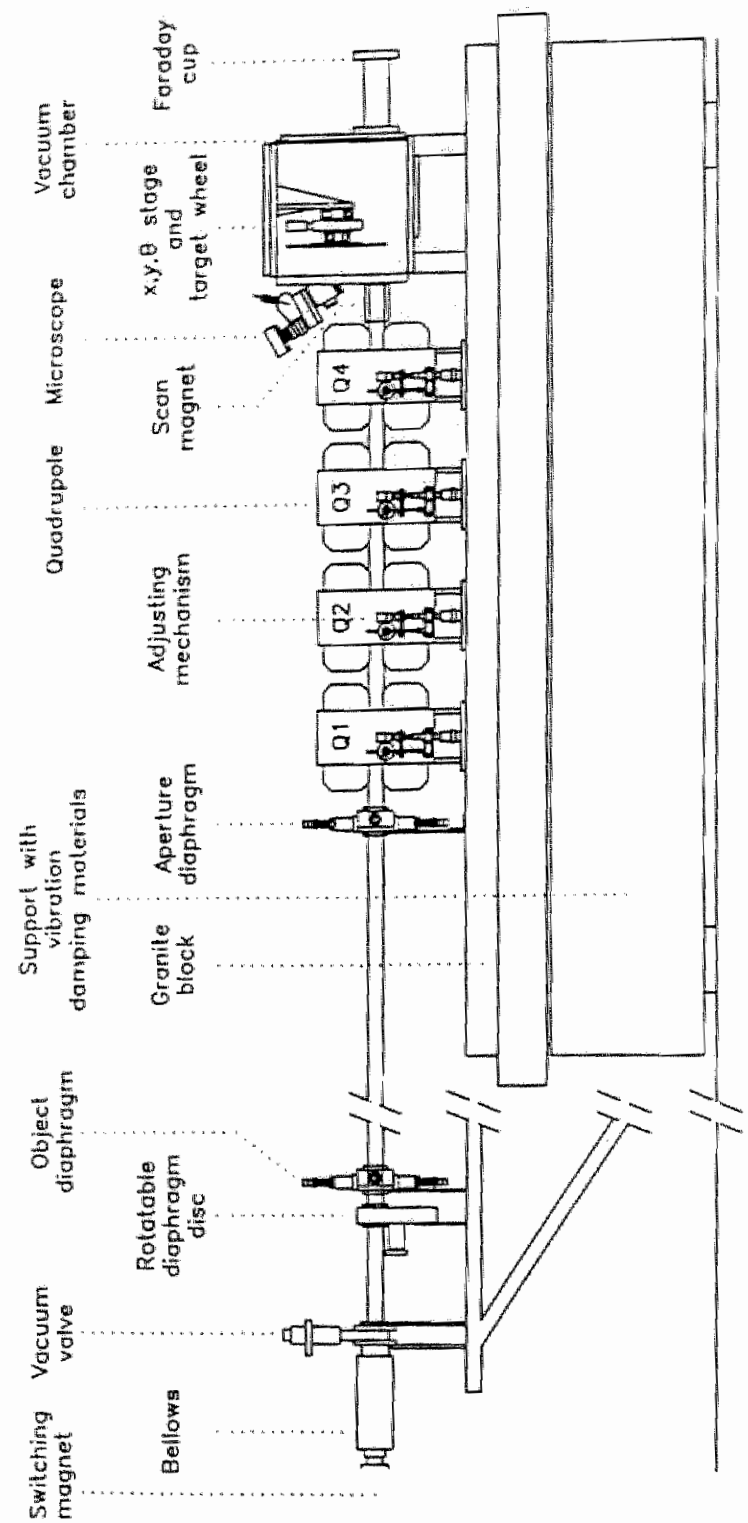

Figure 4. S: Side view of the Eindhoven proton microprobe set-up, Q1, Q2, Q3 and Q4 are magnetic quadrupoles (from (MUTYS)). 
The external cyclotron beam current during microprobe experiments is $30 \mu \mathrm{A}$. After extraction from the cyclotron the protons are transported to the microbeam set-up through a beam transport system. After having traversed a distance of about $30 \mathrm{~m}$ the protons reach a switching magnet, which is used to deflect the beam into one of the four beam lines used for the characterization of materials. One of them is the microprobe set-up. A side view of this set-up, which has a total length of $7.43 \mathrm{~m}$, is shown in Figure 4.1

The proton beam passes through two diaphragms and subsequently is focused to a small spot on the specimen by means of a lens system consisting of four magnetic quadrupoles: The first diaphragm (object diaphragm) acts as an object to be demagnified by the lens system, while the second (aperture diaphragm) limits the divergence of the beam. To prevent the object diaphragm from excessive heating the beam hits an additional diaphragm containing eight different holes of varying size before hitting the object diaphragm. To reduce slit scattering the slit jaws of the object diaphragm as described by Mutsaers [MUT95], have been replaced by cylindrically shaped tungsten carbide slit jaws with a surface rouglness of about 0.01-0.1 $\mu \mathrm{m}$. According to [SCH88] the use of these slit jaws should reduce slit scattering compared to the old slit system. This is especially important for STIM experiments, where slit scattering results in a larger beam spot diameter and appears as a low energy tail in the STIM energy spectrum (see also Section 4.2.2). The position of the slit jaws can be adjusted using micrometers with an accuracy of about $10 \mu \mathrm{m}$. In the near future the slit jaws will be coupled to new micrometers in order to obtain an adjustment accuracy of about 1-2 $\mu \mathrm{m}$.

Mutsaers has improved the focusing properties of the microprobe set-up, and hence he reduced the beam spot size, by improving the focusing properties (the "setting") and by a reduction of the influence of the parasitic aberrations, i.e., abberations due to imperfect constructions and misalignment of the proton microprobe set-up [MUT95]. At present a beam with dimensions of about $3 \times 3 \mu \mathrm{m}^{2}$ with a beam current of about 100 pA can be produced routinely. The determination of the beam-spot size, based upon the detection of protons scattered from a $2.5 \mu \mathrm{m}$ tungsten cross-wire, has been described by Mutsaers for PIXE/RBS experiments [MUT95]. This method fails in case low beam currents are employed or beam spot diameters smaller than $2.5 \mu \mathrm{m}$ are to be measured, as is the case during STIM analysis. Two different methods available to determine the beam spot size during STIM experiments are presented in Section 4.2.2.

The production of a beam spot with the smallest possible dimensions is amongst other things limited by the energy spread of the proton beam. The beam energy spread can be reduced by means of a dispersive system. This system, which is incorporated in the beam-transport system, consists of two horizontal slit systems that are placed before and after two $45^{\circ}$ bending magnets. By imaging the entrance slit (width $1.3 \mathrm{~mm}$ ) on the exit slit (width $1.5 \mathrm{~mm}$ ) theoretically a beam energy spread of $0.1 \%$ should be obtainable with $33 \%$ transmission of beam intensity through the dispersive system [SAN73]. In practice only about $10-15 \%$ of the external beam current is transmitted in case the beam transport system is operated in the dispersive mode. As is shown in Section 4.2 .2 , a significant reduction in beam-spot size is obtained during STIM experiments when the dispersive mode of operation is used. The influence of the dispersive mode on the spot size is less pronounced in case a proton 
beam is employed for elemental analysis purposes, i.e., during PIXE and NBS/NF! experiments. This effect is probably due to the fact that the infuence of chromatic aberration! on the beam spot size is of more significance for a small object diaphragm diameter, as is usec during STM experiments [MAA96].

\section{The target chamber}

The target chamber is equipped with a target wheel which can hold eight different target frames with dimensions of $3 \times 50 \times 50 \mathrm{~mm}^{3}$, which is exactly the size of a standard slide frame. For analysis of biological specimens wet tissue sections are collected on a Formvar foil covering a $010 \mathrm{~mm}$ hole in a plastic sheet (see Fig. 4.2 and Section 5.1). This sheet can be clasped in a slide frame which fits into the target wheel. This target holder construction enables one to pick up sections of large diameter, for example a histological section of the ventricular wall of a rat heart. Moreover, the production of background X-rays by the interaction of the beam halo with the material outside the specimen is strongly reduced in this way.

a
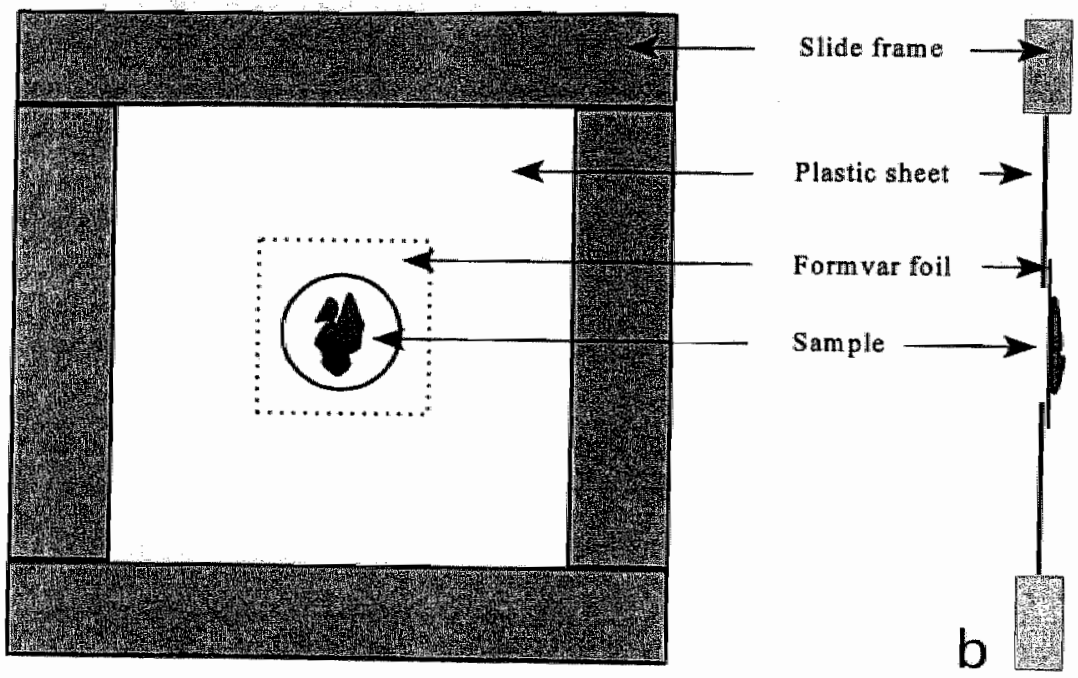

Figure 4.2: Schematic representation of the target holder construction (adapted from (KUTly): a: front wiew; $b$ : side view.

The sample can be positioned with respect to the beam by means of a $x-y$ translation stage with an accuracy of $1 \mu \mathrm{m}$. Beam scanning can be obtained by means of magnetic deflection of the proton beam using a scanning magnet that is located between the last magnetic quadrupole and the target chamber [MUT95]. The frequency at which the beam is scanned across the specimen can be varied with a maximum scan frequency of $10 \mathrm{kHz}$. It should be noted that a 
"scan" frequency of $1 \mathrm{kHz}$ means that 1,000 successive steps are made by the beam within $1 \mathrm{~s}$. In this respect it might be better to use the word "step" frequency instead of scan frequency. For $3 \mathrm{MeV}$ protons in both directions a maximum deflection of about 2 mm with respect to the beam axis can be attained, resulting in a scanned area with maximum dimensions of about $2.8 \mathrm{~mm} \times 2.8 \mathrm{~mm}$.

A smallest step size of $0.174 \mu \mathrm{m}$ was achievable during all experiments described in this thesis. The most important advantages of using a scanning magnet instead of noving the target with respect to the beam are the possibility to perform STIM measurements and the reduction of sample damage in case high scanning frequencles are employed [LLA90]. In addition, the use of high scanning frequencies makes it possible to get a first impression of both areal mass density and elemental distribution of the sample within a few minutes. This enables one to decide whether it is useful to continue the measurement or to analyze another area of the sample.

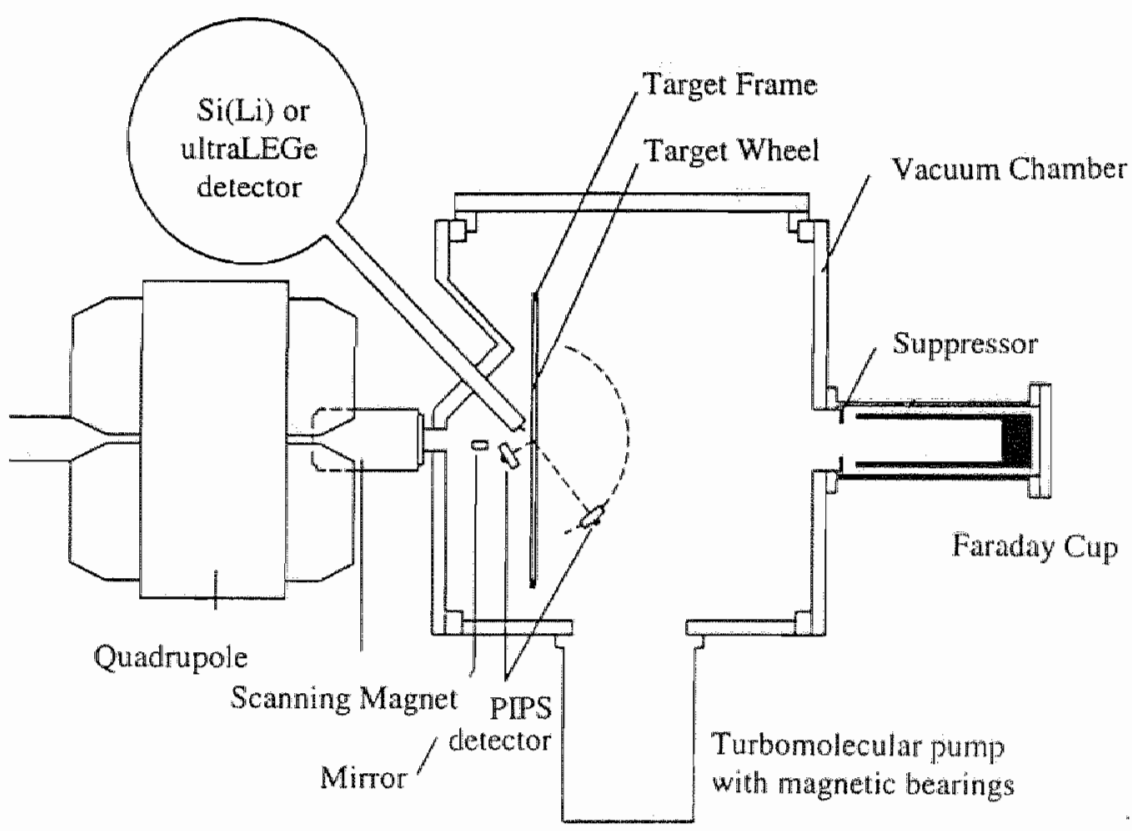

Figure 4.3: Schematic representation of the rarget chamber of the microprobe set-ip from [MUTO5])(PIPS = Passivated Implanted Planar Silicon). 
The target can be visualized using a stereo-zoom microscope via a mirror that is placed inside the target chamber. The use of the mirror requires in a large working distance (low numerical aperture) and a resolution of about $3 \mu \mathrm{m}$. The microscope is coupled to a CCD camera in order to provide visualization of the target on a monitor outside the room where the proton microbeam set-up is located.

The number of protons passing through the sample is detected using a Faraday cup, which is placed behind the target chamber. The Faraday cup can be connected to a Keithley electrometer or a current digitizer (Ortec, Munich, Germany) to measure the beam current during proton bombardinent.

To perform PIXE experiments an X-ray detector is placed close to the target at an angle of $135^{\circ}$ with respect to the direction of the incoming proton beam. Two different $\mathrm{X}$-ray detectors (both manufactured by Canberra, Olen, Belgium) are used in our set-up: a $\mathrm{Si}(\mathrm{Li})$ detector with an area of $30 \mathrm{~mm}^{2}$ and an energy resolution (at Full Width at Half Maximum, FWHM) of $165 \mathrm{eV}$ at $5.9 \mathrm{keV}$ and an ultra-LEGe (Low Energy Germanium) detector with an area of 50 $\mathrm{mm}^{2}$ and an energy resolution of $140 \mathrm{eV}$ FWHM at $5.9 \mathrm{keV}$.

The calibration of both $\mathrm{X}$-ray detectors is presented in the following sub-section. Due to the finite dimensions of the aluminum end-cap of the X-ray detector the minimum distance between the target and the Be-window is about $14 \mathrm{~mm}$. Generally the detector crystal is placed at about $5 \mathrm{~mm}$ behind the Be-window, thus resulting in a maximum detector solid angle of $0.08 \mathrm{sr}$ for the $\mathrm{Si}(\mathrm{Li})$ and $0.14 \mathrm{sr}$ for the ultra-LEGe detector.

Two Canberra PIPS (Passivated Implanted Planar Sillicon) detectors are used to measure the energy of the back- and forward-scattered protons. In order to perform NBS experiments one PIPS detector is placed at a fixed angle of $147^{\circ}$ with respect to the beam axis to detect backscattered protons. The other particle detector is placed on an arc segment which can be rotated without the need to vent the target chamber. In this way forward scattered protons can be detected at angles from $0^{\circ}$ up to $90^{\circ}$ with respect to the beam direction. During NFS experiments the PIPS detector is placed at $45^{\circ}$, whereas the detector is positioned directly behind the sample in ease a bright-field STIM experiment is performed. The angles of detection for both the NBS and NFS detector are chosen such because of the availability of data for the non-Rutherford scattering cross-sections at these angles [AM[93]. Both PIPS detectors have an active area of $100 \mathrm{~mm}^{2}$ and can be translated towards the target at a fixed angle in order to increase the solid angle of detection. Finally a channeltron (X630 CL, Philips, Eindhoven, the Netherlands) is present in order to detect secondary electrons emitted from the sample. As will be shown in Section 4.2.4, secondary-electron detection can be employed either to produce a two-dimensional image of the sample surface or to determine the beam spot size during STIM experiments. The channeltron is positioned at an angle of about $120^{\circ}$ with respect to the beam axis and thus detects secondary electrons ejected from the specimen surface facing the incoming proton beam.

All detectors are placed upon an aluminum sub-frame which can be removed from the target chamber. As a result the position and fixation of the detectors can be changed and other equipment can be added very easily without the need to modify the bottom side of the vacuum chamber. 
The vacuum chamber is evacuated using a $4001 \cdot \mathrm{s}^{-1}$ turbo molecular pump (TMP). This pump is equipped with magnetic bearings to prevent vibration. Normally the pressure reached in the target chamber is about $2 \cdot 10^{-4} \mathrm{~Pa}$. In the beam line of the microprobe, which is pumped with a $501 \cdot \mathrm{s}^{-1}$ TMP (not shown in Fig. 4.1), normally a pressure of about $10^{-3} \mathrm{~Pa}$ is reached. Vibrations of the microprobe set-up are damped as much as possible by making use of bellows between the TMPs and the roughing pumps and by placing part of the microprobe on a granite support with vibration damping materials.

\section{Proton rejection}

During a PIXE experiment backscattered protons can reach the $\mathrm{X}$-ray detector. The $\mathrm{Be}$ window in front of the detector crystal of both X-ray detectors, with a thickness of about 8 $10 \mu \mathrm{m}$, is too thin to stop these protons. As a result, during the measurements backscattered protons also deposit their energy in the X-ray detector, thereby interfering with detected Xrays. This results in worsening of the detector resolution and in an increased background under the characteristic peaks in the X-ray spectrum, especially at low energies (1-2 keV). To prevent these protons from entering the $\mathrm{X}$-ray detector commonly an extra absorber in front of the detector is employed. Using the computer program TRIM [BIE91] it follows that a total Be thickness of $75 \mu \mathrm{m}$ would be required to stop all backscattered protons. However, this is only useful if high energy $X$-rays from heavy elements are to be detected, since the transmission for $\mathrm{X}$-ray photons coming from light elements $(\mathrm{Na}, \mathrm{Mg}$ ) is reduced to less than $1 \%$ when such an absorber is used. An alternative is to veto electronically all incoming events when a proton enters the $X$-ray detector. To achieve this the inhibit signal from the preamplifier of the $\mathrm{X}$-ray detector is amplified and a logical pulse is obtained using a timing filter amplifier (Ortec 474) and a constant fraction discriminator (Ortec 584), respectively. Subsequently this signal is stretched to a width of about 150-200 $\mu$ s using a gate and delay generator (Philips Scientific 794), which is slightly longer than the time interval during which the backscattered protons deteriorate the detector signal. The influence of the proton rejection circuitry on the width of the peaks in the X-ray spectrum was checked using a thin calibration foil, consisting of a thin aluminum layer evaporated on a polycarbonate backing foil. At a beam current of $100 \mathrm{pA}$ the FWHM of the $\mathrm{All} \mathrm{K}_{\text {en }}$-line improved from about $180 \mathrm{eV}$ down to 130 $135 \mathrm{eV}$ when proton rejection was utilized.

\section{Detector calibration}

Both a $\mathrm{Si}(\mathrm{Li})$ - and an ultra-LEGe-detector are available to detect the characteristic X-rays coming from the specimen. The calibration of the Si(Li)-detector has been described by Munnik [MUN94], who determined the thickness of the Be-window (10 $1 \mu \mathrm{m})$, the Au-contact $(0.04 \pm 0.01 \mu \mathrm{m})$ and the $\mathrm{Si}$ dead layer $(0.3 \pm 0.1 \mu \mathrm{m})$ in order to establish the detector efficiency $c\left(E_{X}\right)$ for $X$-ray energies in the range of $1-40 \mathrm{keV}$. These values of the parameters are used in this thesis to calculate the detector efficiency in case the Si(Li)-detector is used (see Chapter 6 and 7).

In addition, Munnik determined the Si-crystal surface area of the Si(Li)-detector [MUN94]. He found that the measured value of $23.3 \mathrm{~mm}^{2}$ deviates strongly from the manufacturer's 
value of $30 \mathrm{~mm}^{2}$, thus resulting in lower detector solid angle. For this reason it was decided to verify the solid angle of detection of the ultra-LEGe detector by determination of both the surface area (manufacturer's value $50 \mathrm{~mm}^{2}$ ) and the distance of the Ge crystal behind the Be detector window.
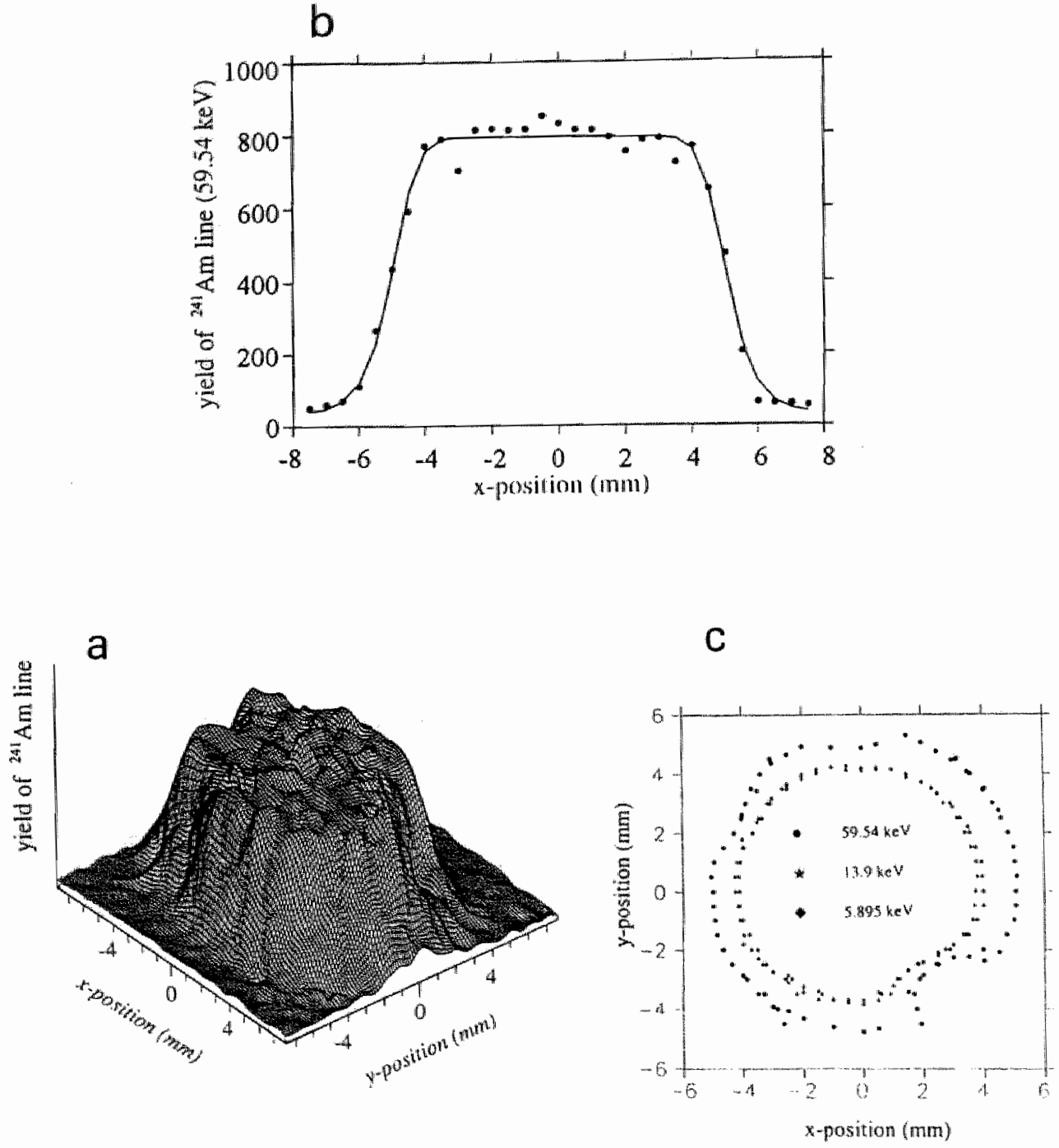

Figture 4.4: Yicld of the $59.54 \mathrm{keV}^{24}$ Am line as a function of the position on the Ge detector crystal (a). The projection of the yied across the line $y=+0.5 \mathrm{~mm}$ is given in (b). Positions at which the yield is $50 \%$ of the fitted maximum value are displayed in (c).

In literature several methods to determine the crystal surface area are described [ORL 89 , HAN73, COH80, CAM84, MUN94]. Most methods are based upon scanming the detector 
surface using a collimated X-ray source. Under the assumption that the surface shape is circular one or two line-scans are sufficient to determine the surface area. These line-scans should cross the centre of the crystal, which either can be determined from an extra line-scan or can be taken as the geometrical centre of the Be window. Some authors, however, found significantly different values for the crystal diameter in horizontal and vertical direction [CAM84, MUN94]. A possible explanation for this effect is a non-uniform thickness of the detector dead layer or misalignment of the detector crystal in the end-cap. A disadvantage of the use of line-scans is that deficient crystal areas outside the scanned pixels are not detected. For this reason in this work the complete ultra-LEGe detector area was scanned using a collimated X-ray source.

The X-ray source, consisting of a ${ }^{241} \mathrm{Am}$ source mounted on a ${ }^{55} \mathrm{Fe}$ source, is collimated using a diaphragm with a hole diameter of $1.00 \pm 0.05 \mathrm{~mm}$. The diaphragm is made of $4 \mathrm{~mm}$ thick brass in order to absorb $99.4 \%$ of the $59.54 \mathrm{keV}$ photons emitted by the ${ }^{241} \mathrm{Am}$ source. The use of a combined $\mathrm{X}$-ray source provides the possibility to determine the active surface of the $\mathrm{Ge}$ crystal for different photon energies in a single measurement.

First the geometrical centre of the crystal is determined by means of line scans in both horizontal and vertical direction. Subsequently the complete crystal surface is scanned using a pattern of $31 \times 31$ positions with a step size of $0.5 \mathrm{~mm}$, corresponding with a scanned area of $15 \times 15 \mathrm{~mm}^{2}$. Every position on the Ge crystal is irradiated during $12 \mathrm{~min}$, resulting in a total measurement time of $192 \mathrm{hrs}$. For each position the net peak intensity of the photo-peaks in the measured energy spectrum is determined using the AXIL program (see also Section 4.3). Figure 4.4a shows the yield of the $59.54 \mathrm{keV}$ line of ${ }^{241} \mathrm{Am}$ as a function of the position on the detector crystal. The yield across the line $y=+0.5 \mathrm{~mm}$ is displayed in Fig. $4.4 \mathrm{~b}$. The points are fitted using the following equation:

$$
Y=\frac{C_{1}}{1+\exp \left(C_{2}+C_{3}\left|x+C_{4}\right|+C_{5}\left(x+C_{4}\right)^{2}\right)}
$$

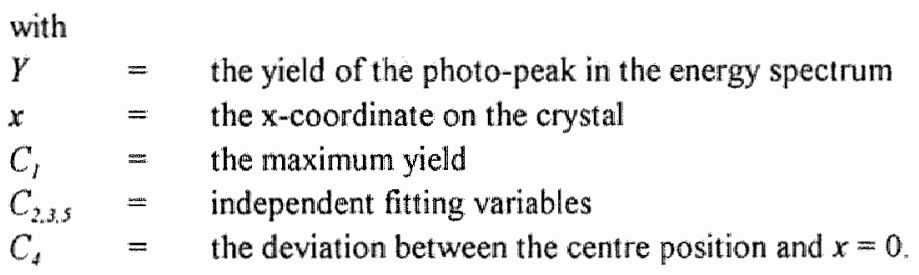

From this fit the two $\mathrm{x}$-coordinates at which $\mathrm{Y}=0.5 \mathrm{C}_{1}$ can be calculated. The same procedure can be applied by regarding the scanned area as the sum of 31 independent linescans in the $y$-direction. All positions at which $Y=0.5 C_{1}$ are displayed in Fig. $4.4 \mathrm{c}$. The active area of the crystal, defined as the area enclosed by the points depicted in the figure, is given in Table 4.1 for three different photon energies. 
Table 4.1 Total active crystal stufface area $A_{\text {as: }}$ of wha-LEGe and Si(Li) detectar for different photon energies. The manufacturer's walues for the detector surface areas are $50 \mathrm{~mm}^{2}$ for the LEGe and $30 \mathrm{~mm}^{2}$ for the Silli) detector, respectively.

\begin{tabular}{ccc}
\hline photon energy $(\mathrm{keV})$ & $\mathrm{A}_{\mathrm{act}}$ LEGe $\left(\mathrm{mm}^{2}\right)$ & $\mathrm{A}_{\mathrm{act}} \operatorname{si}(\mathrm{Li})\left(\mathrm{mm}^{2}\right)$ \\
\hline 5.895 & $47.8 \pm 0.9$ & $22.6 \pm 0.8$ \\
13.9 & $50.6 \pm 0.9$ & $25.5 \pm 0.8$ \\
59.54 & $74.4 \pm 1.0$ & n.d. \\
\hline
\end{tabular}

In contrast with the $S(\mathrm{Li})$ detector, the value for the measured crystal surface area of the ultra-LEGe detector of $47.8 \mathrm{~mm}^{2}$ for $5.985 \mathrm{keV} X$-rays agrees reasonably well with the manufacturer's value ( $\left.50 \mathrm{~mm}^{2}\right)$. The small discrepancy between the measured and stated value for $A_{a t}$ can be attributed to the deficient spot in the bottom right corner of the crystal. After opening the detector it appeared that the high voltage contact was screening the detector crystal at this particular position. For the $59.54 \mathrm{keV} \gamma$-rays a much larger crystal surface area is found. This can be explained by the fact that the collimator in front of the Ge crystal, which consists of a $0.5 \mathrm{~mm}$ thick. Al ring with an inner diameter of $8 \mathrm{~mm}$ (data from manufacturer), is almost transparent for high energy photons such as the $59.54 \mathrm{keV} \gamma$-rays.

The distance between the $X$-ray source and the average interaction depth in the crystal $r$ is composed of the distance between the source and the Be-window $d_{l}$, the distance between the Be-window and the detector surface $d_{2}$ and the average penetration depth of the X-rays in the crystal $z$. The total distance $r$ was determined by measuring the $X$-ray yield at different positions of the $\mathrm{X}$-ray source with respect to the detector. The measurement was performed under ambient pressure. For this reason the measured yield was corrected for the absorption of $5.895 \mathrm{keV} X$-rays in air (see Fig. 4.5). After extrapolation the distance between the Bewindow and the detector erystal was found to be $d_{2}=11.5 \mathrm{~mm}$. The average interaction depth $z$ for $5.895 \mathrm{keV} X$-ray photons amounts to $8.3 \cdot 10^{-3} \mathrm{~mm}$ and thus is negligibly small compared with the total distancer.

The large value of $\mathrm{d}_{2}$ has significant consequences for the detector sollid angle $\Omega$. Using the measured values for the Ge-crystal surface area $\left(47.8 \mathrm{~mm}^{2}\right)$ and the distance between the Bewindow and the Ge-crystal (11.5 mm), a maximum detector solid angle of $0.074 \mathrm{sr}$ is found if a minimum distance between the target and the Be-window of $14 \mathrm{~mm}$ is assumed. This means that, despite the larger area of the Ge-detector crystal, the maximum solid angle of detection can be obtained in case the Si(Li)-detector is used instead of the ultra-LEGe-detector. At the time the measurements described in Chapter 5 were performed this information was not available. Therefore, during the experiments presented in Chapter 5 the ultra-LEGe-detector is still used for the detection of X-rays, whereas the Si(Li)-detector is used for all measurements described in the subsequent chapters. In Chapter 5 for the ultra-LEGe the data from the manufacturer are taken to determine the detection efficiency and hence to calculate the values for the elemental content. 


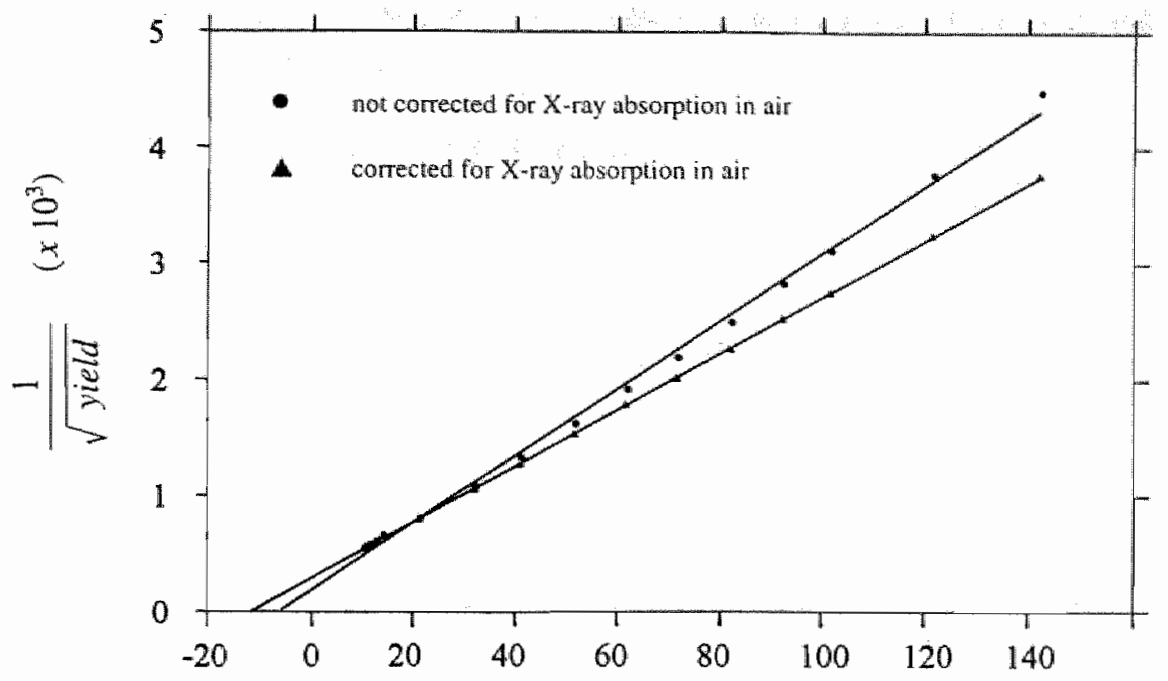

distance between Fe-source and Be-window (mm)

Figure 4.5 Yield of 5.895 keV $X$-rays as a finction of the distance $d_{1}$ between the ${ }^{35} F$ X-ray source and the Be-window. Since the yield is proportional to $1 / \mathrm{r}^{2}$, in the figure If Syield is displayed. Both the yield corrected and non-corrected for the absorption of the $X$-rays in air are displayed (from [IAN95]).

\subsection{Imaging techniques}

The proton microprobe experiments are performed using thin, freeze-dried sections of cardiac tissue cut at a thickness of about $10 \mathrm{\mu m}$ (Section 5.1). In the case of PIXE microanalysis of biological cells precise localization of the scanned area is required, particularly when local differences in elemental concentration are expected within the tissue section. In this section four different techniques that can be used to localize individual cells within such a section are presented, i.e., light microscopy (4.2.1), STIM (4.2.2), electron microscopy (4.2.3) and the detection of secondary electrons (4.2.4). Both STIM and secondary electron detection are applied in the proton microprobe during the bombardment of the sample with protons, whereas both the light and electron microscope can be employed separately to study the histological structure of the sample prior to analysis in the proton microprobe set-up.

\subsubsection{Light microscopy}

Prior to the analysis in the proton microprobe thin tissue sections, cut at a thickness of about $10 \mu \mathrm{m}$ in a cryostat microtome (see Section 5.1), can be analyzed using a light microscope. 
This provides the possibility to study the morphology of the tissue and hence to localize the individual cells. In our case both the light microscope mounted in the proton microprobe setup or a separately placed microscope can be used. However, due to the low numerical aperture of the former, which results in a poor resolving power, it is very difficult to find areas suitable for scanning PIXE experiments or to relate the measured elemental distributions to the histological details of the specimen. As a result the light microscope of the proton microprobe set-up is not suitable to visualize the individual cells within the tissue sections to be analyzed.
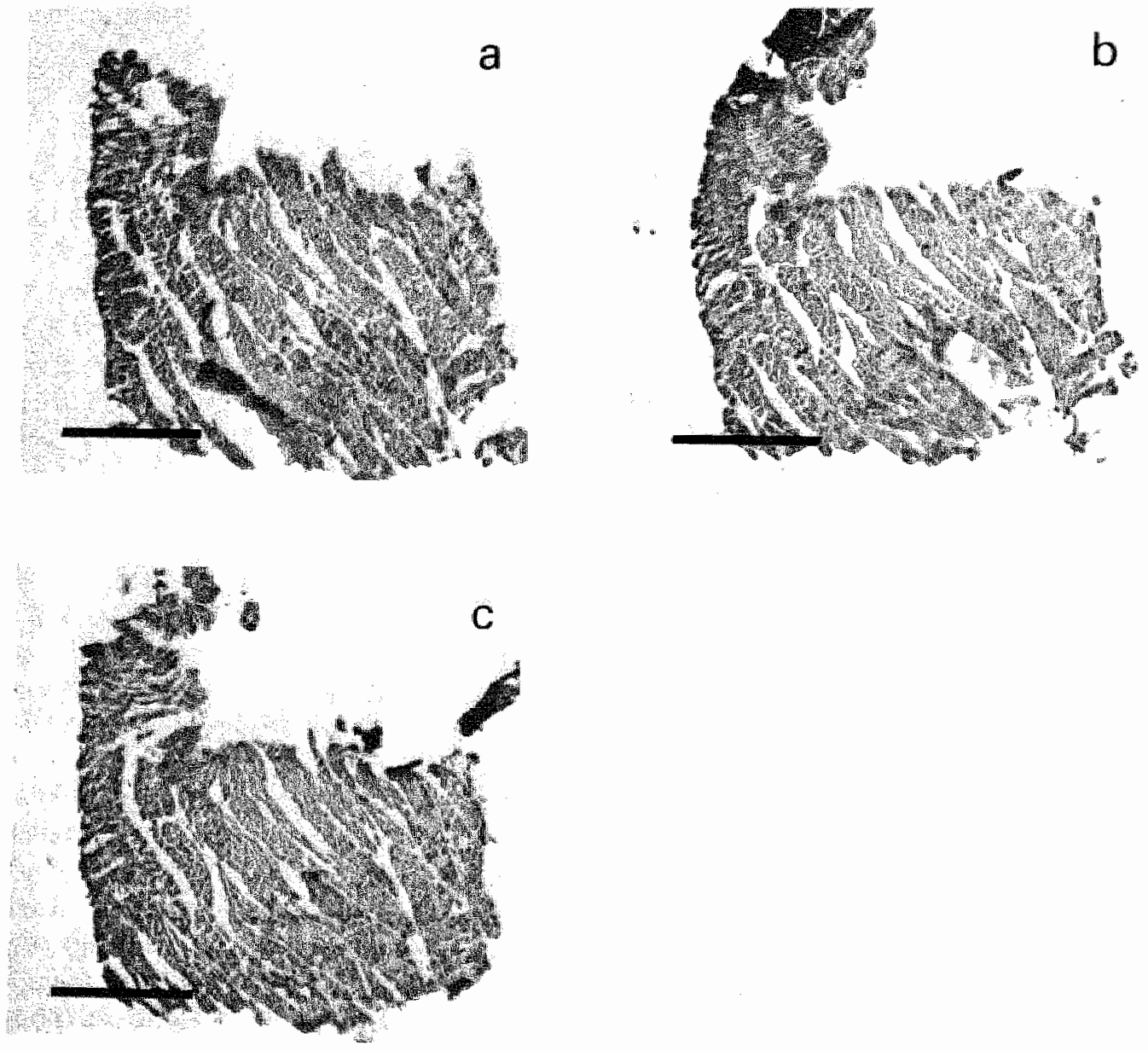

Figure 46: Ligh microscope pictures of three toludine blue stained sections of cardiac tissue. The third (a) flfth (b) and seventh (c) section are shown. respectively. Sections were cut at a thichness of $10 \mu \mathrm{m}$. Note the reproducibility of the tissue structure of the various sections. Bars represent $0.5 \mathrm{~mm}$. 
In summary the following protocol is followed: in case the biological sample is to be analyzed in the proton microprobe set-up for the assessment of the elemental content (i) whstamed sections are used, (ii). poor resolution from the light microscope mounted th the proton microprobe set-up is obtained and (iii) no cells are visible. However, in stamed adjacent sections that are analyzed in a separately mounted light microscope cells are wisible. By comparison of the unstained section with the stained adjacent sections a first selection of the area of the unstained section to be analyzed by the proton microprobe can be made. Subsequently, the STIM technique is used to visualize the individual cells within this area.

It can be concluded that the use of stained adjacent sections makes it possible to localize the individual cells within the tissue prior to proton microprobe analysis and facilitates the selection of a suitable area of the tissue section to be analyzed by the proton probe. Both STIM and the detection of secondary electrons, which are described in the following sections facilitate to visualize the individual cells within the unstained freeze-dried cryosection itself. For this reason during this study these techniques were incorporated into the proton microprobe analytical procedure.

\subsubsection{Scanning Transmission Ion Microscopy (STIM)}

During STIM analysis, a solid-state particle detector measures the energy loss of the protons after passage through the specimen. This loss can be converted into the local areal mass thickness for every position irradiated by the scanning proton beam. By placing the particle detector under an angle of $0^{\circ}$ with respect to the beam direction (bright field STIM) a detector efficiency close to $100 \%$ is obtained, since almost each proton is detected. As a result a beam current of $\sim 1 \mathrm{fA}$, corresponding to about $10^{4}$ protons $/ \mathrm{s}$, is sufficient to obtain an areal mass thickness picture of the specimen after only a few minutes of irradiation. This underlines the high efficiency of STIM compared to PIXE, which requires a few hours measurement time and a $10^{5}$ times higher beam current. Furthermore, the low beam current employed during STIM anallysis offers the possibility to reduce the size of the diaphragms. Theoretically a reduction in the diaphragm diameters should result in a smaller beam spot size and thus a better spatial resolution. In analysis of biological tissue an improvement in spatial resolution is of great importance in order to visualize (sub)cellular structures within a freeze-dried tissue section. Since the use of $\$ 2.5 \mu \mathrm{m}$ tungsten cross-wire fails to determine the beam spot size in case low beam currents are employed or beam spot diameters smaller than $2.5 \mathrm{fm}$ are to be measured, the next sub-section is devoted to the determination of the beam dimensions during STIM experiments.

\section{Determination of beam dimensions}

Two different test objects were used to determine the beam spot size during STIM experiments, i.e., polystyrene latex spheres and a test chip. The use of the test chip is discussed in Section 4.2.4. The latex spheres (Agar, Stansted, England), that are normally used as a size check in electron microscopy, are available with diameters ranging from 0.091 up to $1.090 \mu \mathrm{m}$ with very small standard deviation on the particle size. In our study latex 
spheres with a diameter of either 0.497 or $1.090 \mu \mathrm{m}$ were used. A small droplet of a $0.1 \%$ $(w / w)$ suspension of latex spheres was allowed to dry on a Formvar backing foil covering a copper finder grid (type H-2, Agar, Stansted, England). This 200 mesh finder grid contains micron-sized letters attached to the copper bars that can easily be identified by the light microscope present in the microprobe set-up. However, due to the low numerical aperture of the light microscope it is not possible to visualize the individual latex spheres. Therefore, the position of the latex spheres with respect to the letters present in the grid was determined using a Philips CM-12 Scanning Transmission Electron Microscope (STEM) prior to proton microprobe analysis.

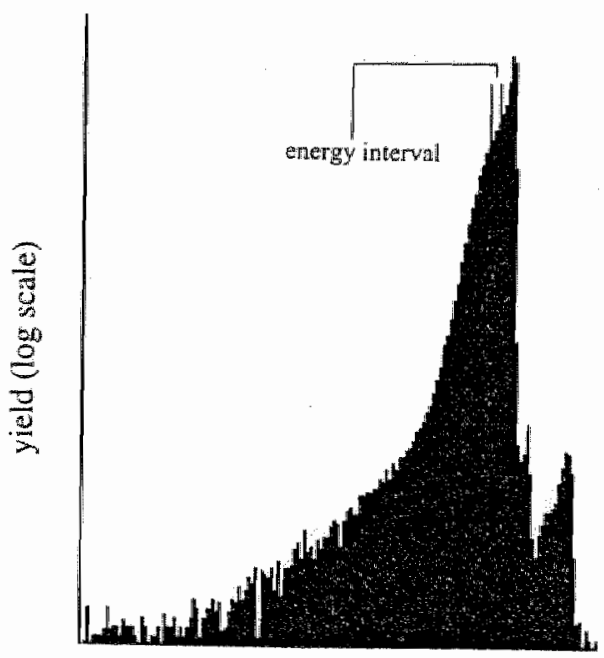

energy of transmitted protons (keV)

Figure 4.7: Energy spectrum of protons transmitted throwgh a $1 / \mu m x, 1 /$ um area of Formvar foil stupporting two $1.090 \mu \mathrm{m}$ diameter latex calibration spheres. Protons that passed through borh foll and sphere formed she shoulder on the low energy side of the main distribution. Events with energies in the energy interval indicated in the figure were used to form the STM image displayed in Fig. 4.86 .

Figure 4.8 a shows the result from the STEM analysis of the latex spheres, whereas the STIM image of the same specimen is given in Fig. $4.8 \mathrm{~b}$. The proton beam was operated in the dispersive mode in order to reduce the influence of beam energy spread on the spot size. Due to the small difference in mass (and thus a small difference in energy loss experienced by the proton beam) a rather long measurement time is needed to distinguish the latex spheres from the Formvar backing foil (see also Section 2.2.2). 

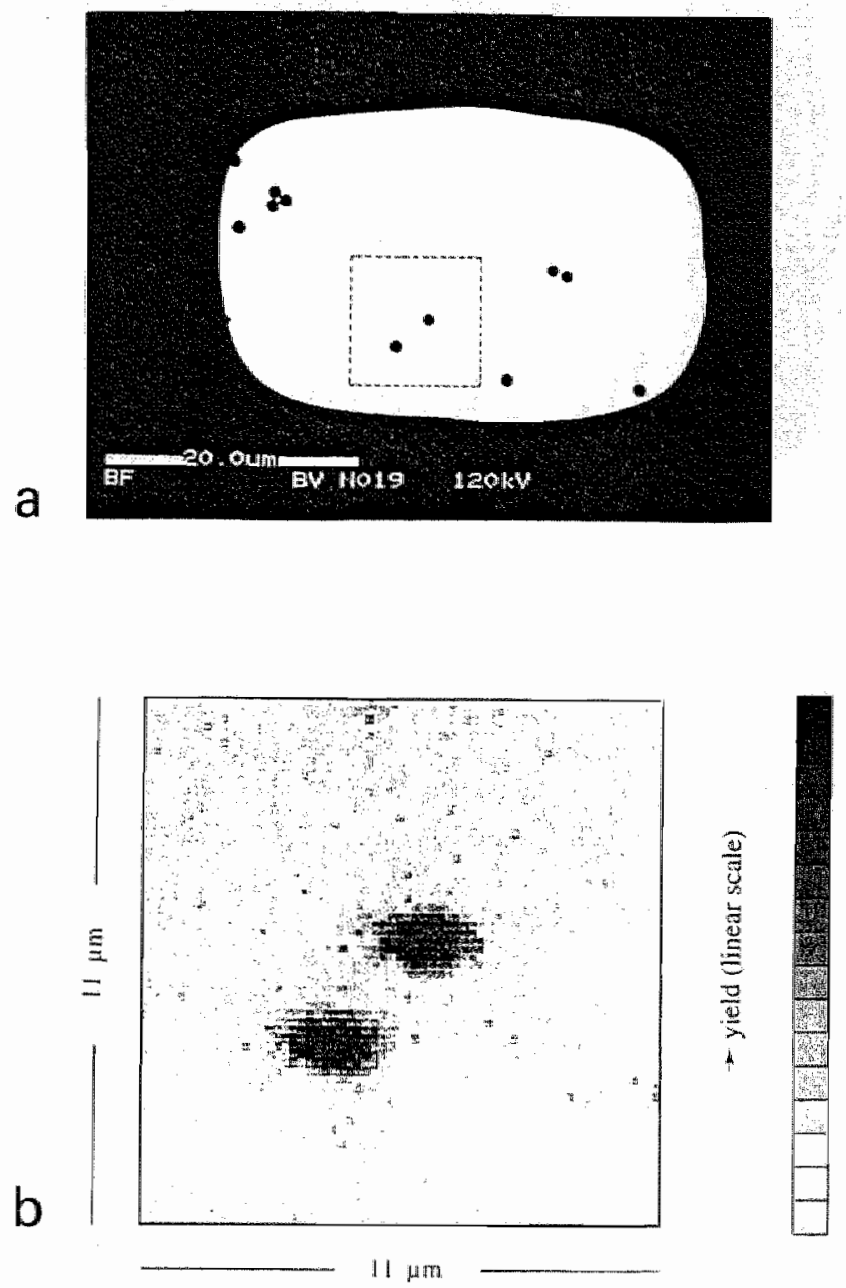

Figure 4.8: $\quad$ STEM (a) and STLM-image (b) of the same latex spheres covering a formut backing foil. The scanning area displayed was $M \mu \mathrm{m} \times \| \mu \mathrm{m}$. The nominat diameter of the latex spheres is $1.090 \mu \mathrm{m}$.

Figure 4.9 shows the projection of the proton yield in a small energy window as a function of the horizontal and vertical beam displacement, respectively. The FWHM of the beam diameter was estimated by subtracting in quadrature the fwhm of the latex spheres from the fwhm of the measured signal. The resulting beam dimensions are $1.5 \mu \mathrm{m}$ in the $x-$ and $1.1 \mu \mathrm{m}$ in the $y$-direction. This is significantly better than the minimum beam spot size of about $3.0 \mu \mathrm{m} \times 3.0 \mu \mathrm{m}$ obtainable during PIXE and NBS/NFS experiments. 


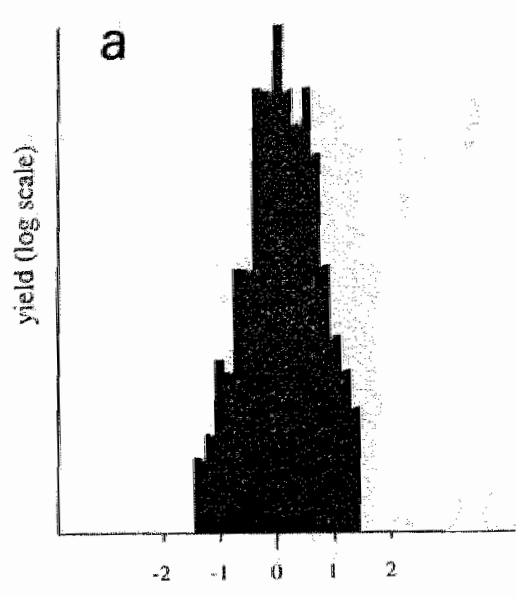

displacement in $x$-dinection ( $\mu$ m)

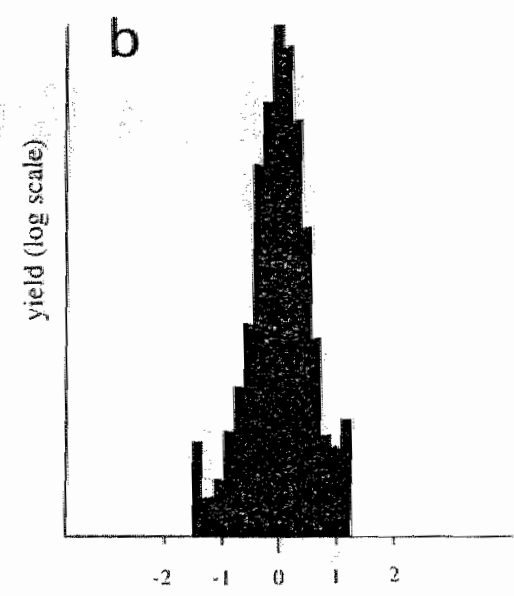

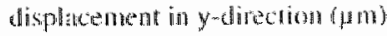

Figure 4.9: Yield versus horizontal (a) and vertical (b) bean displacement for protons in the energy interval indicated in Fig. 4.7. The peak width implies, after subtracting the FWHM of the sphere according to the "quadrate rule", a FWMM beam diameter of $1.5 \mu \mathrm{m}$ in the $x$-direction and $1.1 \mathrm{~mm}$ in the $y$-direction, respectrvely.

\section{Localization of individual cells in tisste using STIM}

In the preceding sub-section it has been demonstrated that the use of the STIM technique results in a substantial improvement in spatial resolution as compared with PIXE analysis. However, in order to apply the STIM technique to localize individual cells it is also required that differences in areal mass density within a freeze-dried section of biological tissue exist, since STIM is based upon the measurement of energy loss of protons that pass through the sample. This was further tested by using a freeze-dried section from a rat heart. The section was cut at a thickness of $10 \mu \mathrm{m}$ and was collected on a thin Formvar backing foil. Subsequently the tissue section was allowed to freeze-dry in a dessicator. The sample preparation procedure as used in this type of work will be described in more detail in Section 5.1. In order to allow inspection of the morphological structure of the tissue section prior to STIM analysis, the freeze-dried tissue section was stained using toluidine blue as indicated before.

Figure 4.10 shows the result of a STIM measurement of a $3 \mu \mathrm{m}$ thick freeze-dried cryosection of a rat heart. The beam diameter during the experiment was estimated to be 0.5 $1.0 \mu \mathrm{m}$ based upon STIM analysis of a test specimen (see Section 4.2.4). The beam was scanned over an array of $256 \times 256$ pixels with a step size of $0.35 \mu \mathrm{m}$, covering an area of about $90 \times 90 \mu \mathrm{m}^{2}$. In Fig. 4.10a areas with a high proton energy loss are depicted, whereas Fig. $4.10 b$ shows the areas with a small proton energy loss These pictures represent the thicker and thinner parts of the sample, respectively. Since the STIM analysis was performed using a toluidine blue stained section, the STIM pictures can be compared with a light 
microscope photograph (Fig. $4.10 \mathrm{c}$ ) representing the same area that was scanned by the proton beam. The cells and their boundaries are clearly visible in both the light-microscope and STIM pictures with a one to one relation.
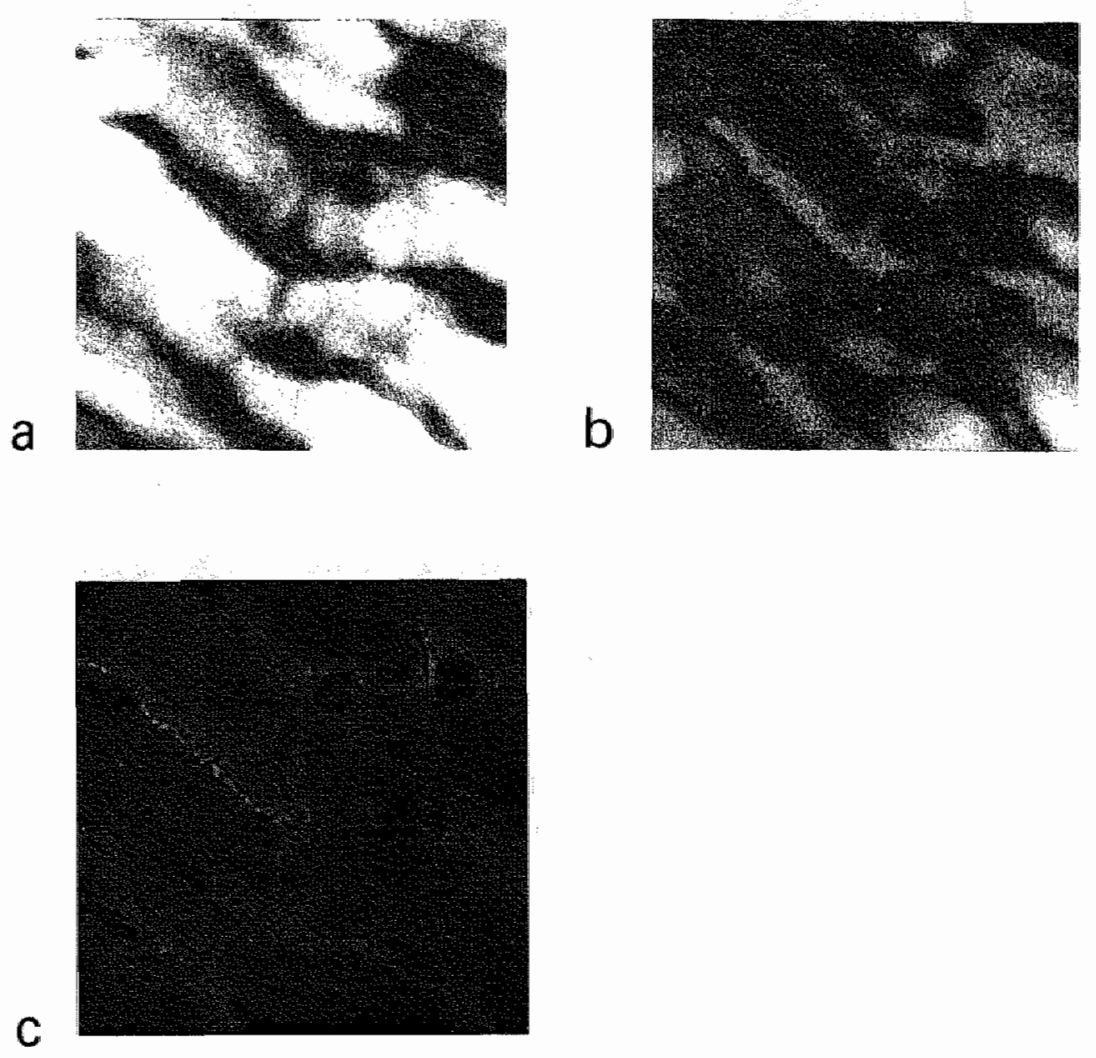

Figure 4. 10." Two STIM pictures (a and b) and a light microscope picture (c) of the same area (about $90 \times 90 \mu \mathrm{m}^{2}$ ) of a myocardial nisswe section stained whth rolwidine bhe. Figure 4.10a represents the protons that suffered a small energy loss while traversing the specimen (thimner parts of the specimen), whereas the high energy loss prowons (thicker areas) are displayed in Fig. 4.70b. 
These results show that, in addition to PIXE, in the same experimental set-up STMM can be employed to localize indiwidual cells within freeze-dried tissue sections. This has also some practical benefits, since this makes it possible to successively perform PIXE and STIM experiments in the proton microprobe set-up. It was shown in Chapter 2 that STIM can also be used to determine the local areal mass density. In this study, however, the STIM technique has only be used for imaging purposes. As will be shown in the next chapters, this offers the possibility to investigate the cell-to-cell variation in elemental concentrations in both healthy and injured cardiac tissue.

\subsubsection{Ellectron microscopy}

Although the use of the STM technique may result in a significant improvement of the spatial resolution, the electron microscope remains better for imaging purposes. For this reason the electron microscope should be used in case subcellular structures have to be visualized. Therefore, in our study the electron microscope was employed to examine the histological ultrastructure of freeze-dried cryosections as well as to trace and recognize artefacts introduced during the complete process of sample preparation (see also Chapter 5). One should note, however, that the superior spatial resolution of the electron microprobe refers to ultrathin specimens with a thickness of about $50-150 \mathrm{~mm}$. For thicker samples, such as used for proton microprobe analysis, the resolution of the electron microprobe becomes much worse due to scattering of the electrons. As a result the electron beam broadens into a pear-shaped volume with an effective lateral resolution roughly the same as the sample thickness. The use of ultrathin specimens for imaging purposes presents no problems. However, elemental analysis of such thin samples with good resolution and low limit of detection is very difficult because of the small mass of the elements encountered by the particle beam (see also Chapter 3).

In this study the quality of the histological preservation of freeze-dried myocardial sections was assessed using a Philips CM-12 electron microscope. The microscope was operated in the scanning transmission (STEM) mode at $120 \mathrm{kV}$ and all measurements were carried out with the specimen at ambient temperature. The sections were cut at a thickness of about 100 . $150 \mathrm{~nm}$. In case no information about elemental concentrations had to be obtained, vapour fixation was applied to stabilize the section and to improve contrast. All sections were fixed after freeze-drying using osmium vapour. To further improve the stability under electron bombardment the sections were coated with a thin carbon layer prior to analysis.

Since the CM-12 electron microscope is equipped with an $\mathrm{Si}(\mathrm{Li})$-detector, next to histological analysis the CM-12 was also used to perform $X$-ray microanalysis of ultrathin sections (see Chapter 5). Despite the fact that in this case the sections were not stained in order to prevent artefacts, the mitochondria could be easily recognized. This enables one to determine possible differences in concentration between the mitochondria and the surrounding tissue. This is a major benefit, since it is not possible to visualize subcellular structures with our proton microprobe. However, because of the relatively poor detection limit of EPMA as compared with PIXE only the concentration of those elements which are rather abundant in 
the sample can be determined. It thus can be concluded that both techniques have their specitic merits and limitations, and thus are proper candidates for complementary use

\subsubsection{Secondary electron imaging}

An alternative method for imaging freeze-dried tissue sections in the proton microprobe set-up using the STIM technique is to detect secondary electrons emitted from the sample during irradiation with the proton beam. Due to the short range of the electrons inside the sample only secondary electrons produced at the specimen surface can escape from the specimen. As a result the number of detected electrons at each position of the scan pattern can be monitored to produce an image of the specimen surface. Since the channeltron used in our set-up is placed at an angle of about $120^{\circ}$ with respect to the incoming proton beam, only ellectroins emitted in backward direction are detected. Therefore, in contrast with STIM, secondary electron detection can be used to provide an image of the surface area from specimens that are thick enough to stop the proton beam entirely.

This property can be used to determine the beam spot size during STIM analysis using a test chip. This chip, developed by Philips (Eindhoven, The Netherlands) for testing lithographic instruments, consists of a $1 \mathrm{~mm}$ thick $\mathrm{Si} / \mathrm{SiO}_{2}$ substrate with $100 \mathrm{~nm}$ thick TiW patterns. The dimensions of the TiW structures range from 0.50 to $2.00 \mu \mathrm{m}$ with an accuracy of about $0.01 \mu \mathrm{m}$, as was assessed from analysis in a Scanning Electron Microscope (SEM).

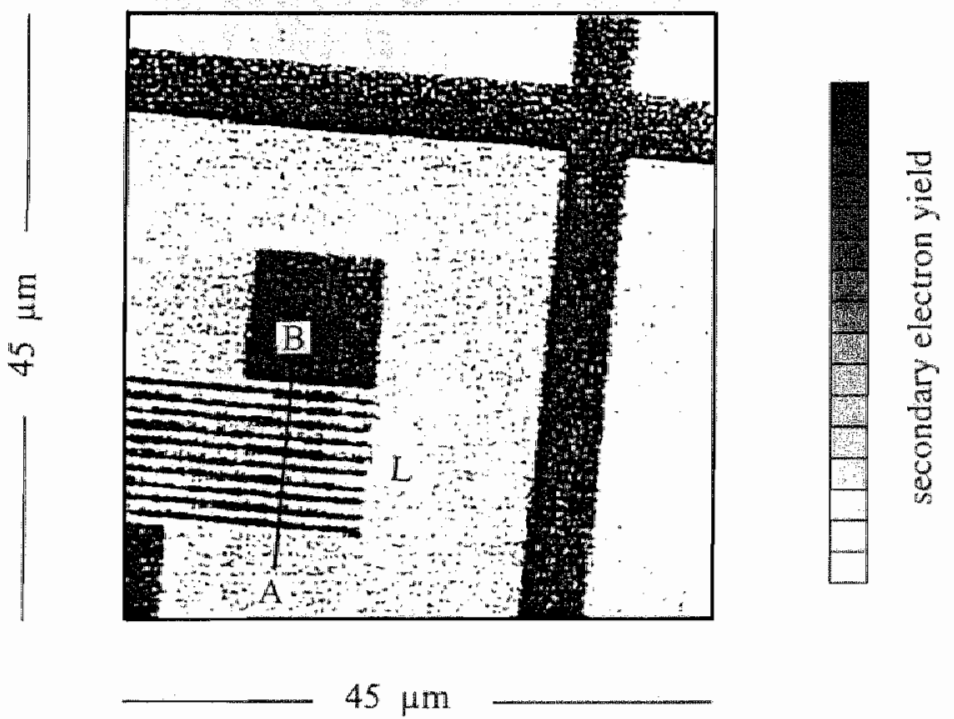

Figure 4.11: Secondary electron image of test specimen. The widh of the horizontal lines (D) is $1.10 \pm 0.01,4 m$, as determined from analysis fin a Samming Electron Microscope. The rotal area scanned by the beam is $45 \times 45 \mathrm{~mm}^{2}$. Addirinally the beam was sommed along the line A-B (see Fig. 4.12). 
In Figure 4.11 the secondary electron image of the test specimen is given in case this specimen is irradiated with a proton beam. The size of both object and aperture diaphragm during the measurements was $0.06 \times 0.08 \mathrm{~mm}^{2}$, resulting in a beam current of about 1000 protons $/ \mathrm{s}$. The values of these settings correspond to those applied during STTM experiments. The beam was scanned across the specimen covering an area of about $45 \times 45 \mu \mathrm{m}^{2}$ with a step size of $0.174 \mu \mathrm{m}$. Using a Philips SEM the width of the layers visible in the lower left corner was determined to be $1.10 \pm 0.01 \mu \mathrm{m}$.

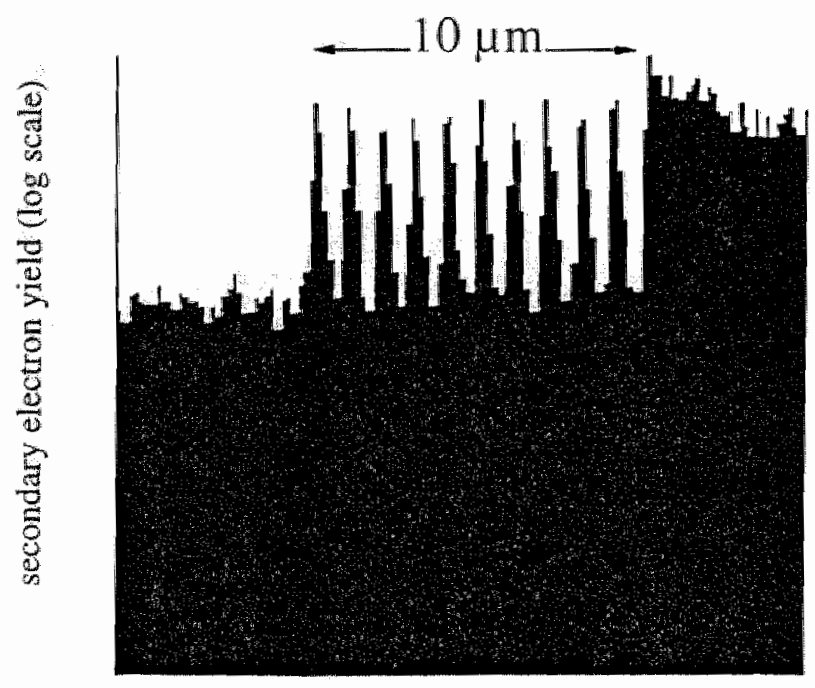

A

B

Figure 4.12. Secondary electron yidd versus beam displacement along the line A-B in Fig. \$.11. After subtracting quadratically the FWHA of the layer a FWHM beam size in the $y$ direction of $0.7 \pm 0.2 \mu m$ is foumd.

Figure 4.12 shows the secondary electron signal which is obtained when the proton beam is scanned across the line AB. The measured signal is deconwoluted with the width of the layers using the program ENTROPY 2 [MAJ96]. The resulting beam dimensions are $1.4 \times 0.7 \mu \mathrm{m}^{2}$ with an accuracy of about $0.2 \mu \mathrm{m}$ in both directions. This implicates that in the proton microprobe sel-up as employed for the STIM technique a significant reduction in beam spot size is achieved when compared to the beam dimensions during PIXE analysis $\left(3 \times 3 \mu \mathrm{m}^{2}\right)$.

\subsection{Data acquisition and data analysis}

\section{Dorta acquisition}

The introduction of a scanning magnet and the need for the possibility to measure simultaneously with more than one detector made a new data-acquisition system necessary. 
This system has been designed and built by Mutsaers [MUT95]. The data generated during a microprobe experiment can be accuired in two ways, i.e., in spectrum mode or in list mode. In spectrum or histogram mode both $X$-ray and NBSNFS spectra are acquired during a period long enough to obtain a statistically meaningful result. In this case after each measurement at a certain position has been finished the $x-y$ translation with stepper motors is used to move the sample to another position. Generally the spectrum mode is used when spectra are to be obtained from only one or a few positions.

In case an elemental distribution is to be obtained the beam is scanned across the specimen. When a scanning frequency of $1 \mathrm{kHz}$ is employed, the proton beam dwells about $1 \mathrm{~ms}$ at each position in the scan pattern before moving to the next position. For this reason the scan pattern should be repeated many times in order to obtain an acceptable total measurement time per pixel. In this case the data are acquired in list mode (event by event), i.e, each detected $X$ ray or proton (energy event) nust be related to the position at the target where it came from. The events from different detectors are labeled with the $(x, y)$-position of the beam on the target. The list mode data are stored in two series of files, one containing the measured energy values and the other the corresponding $(x, y)$-position. Each file represents a certain time interval of the experiment. The duration of the time interval after which these files are written can be adjusted. This enables one to monitor signals as a function of time, which is very valuable in studies of sample damage due to proton bombardment. The monitoring of the data and the data-analysis are discussed below.

The real-time data-acquisition and the experiment control is carried out by PhyDAS (Physics Data Acquisition System). This data-acquisition system consists of a Phybus part and a VME part. Phybus contains the experimental interfaces ( $\mathrm{ADC}$ 's, stepper motor controller's, etc.) needed for the control of the experiment and acquisition of the list mode data. In the Phybus part 3 MCA's (Multi Channel Analyzers) are present that can be used simultaneously to process the events coming from 3 different detectors. One of the main tasks of the VME part of PhyDAS, which contains a Motorolla 68030 microprocessor, is to control the measurement and to take care of the transport of the data acquired by the Phybus part to a DEC/ALPHA-AXP workstation. This workstation is used for data storage on a 1 Gbyte disk, data monitoring and off-line analysis.

\section{Data monitoring and data awalysis}

During acquisition the data can be monitored on-line making use of the data monitoring program COLUMBUS [ZWTJ93, MUT95]. This program contains a database in which all relevant experimental information, such as the detector configuration, amplifier paraneters and name and date of experiments are stored. The database also contains the parameters that define the graphical representation of the acquired data. In general two types of graphics are used, namely (i.) the energy spectrum of the sum of a number of points in a preselected part of the area hit by the scanning proton beam or (ii.) a spatial distribution of the number of X-rays (PIXE), protons (NBS/NFS and STIM) or electrons (secondary electron detection) dletected in a predefined energy interval withim the energy spectrum. The intensity of number of detected X-rays or particles is expressed in either a black and white scale or full colour scale. 
In Figure 4.11 the secondary electron inage of the test specimen is given in case this specimen is irradiated with a proton beam. The size of both object and aperture diaphragm during the measurements was $0.06 \times 0.08 \mathrm{~mm}^{2}$, resulting in a beam current of about 1000 protons $/ \mathrm{s}$. The walues of these settings correspond to those applied during STIM experiments. The beam was scanned across the specimen covering an area of about $45 \times 45 \mu^{2}$ with a step size of $0.174 \mu \mathrm{m}$. Using a Philips SEM the width of the layers visible in the lower left corner was determined to be $1.10 \pm 0.01 \mu \mathrm{m}$.

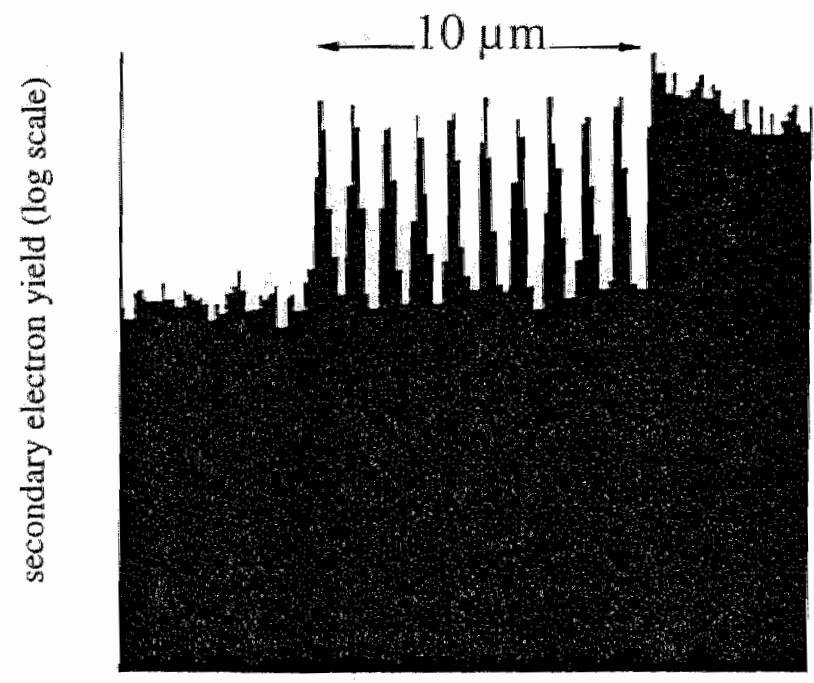

A

B

Figure 4.12: Secondary electron yield versts bean displacement along the line A-B in Fig. 4.11. After subtracting quadratically the FHAM of the layer a FWHM beam size in the $y-$ direction of $0.7 \pm 0.2 \mu \mathrm{m}$ is found.

Figure 4.12 shows the secondary electron signal which is obtained when the proton bearn is scanned across the line AB. The measured signal is deconvoluted with the width of the layers using the program ENTROPY 2 [MAJ96]. The resulting beam dimensions are $1.4 \times 0.7 \mathrm{~mm}^{2}$ with an accuracy of about $0.2 \mu \mathrm{m}$ in both directions. This implicates that in the proton microprobe set-up as employed for the STIM technique a significant reduction in beam spot size is achieved when compared to the beam dimensions during PIXE analysis $\left(3 \times 3 \mu \mathrm{m}^{2}\right)$.

\subsection{Data acquisition and data analysis}

\section{Data acquisition}

The introduction of a scanning magnet and the need for the possibillity to measure simultaneously with more than one detector made a new data-acquisition system necessary. 
This system has been designed and built by Mutsaers [MUT95]. The data generated during a microprobe experiment can be acquired in two ways, i.e., in spectrum mode or in list mode. In spectrum or histogram mode both X-ray and NBS/NFS spectra are acquired during a period long enough to obtain a statistically meaningful result. In this case after each measurement at a certain position has been finished the $x-y$ translation with stepper motors is used to move the sample to another position. Generally the spectrum mode is used when spectra are to be obtained from only one or a few positions.

In case an elemental distribution is to be obtained the beam is scanned across the specimen. When a scanning frequency of $1 \mathrm{kHz}$ is employed, the proton beam dwells about $1 \mathrm{~ms}$ at each position in the scan pattern before moving to the next position. For this reason the scan pattern should be repeated many times in order to obtain an acceptable total measurement time per pixel. In this case the data are acquired in list mode (event by event), i.e., each detected Xray or proton (energy event) must be related to the position at the target where it came from. The events from different detectors are labeled with the $(x, y)$-position of the beam on the target. The list mode data are stored in two series of files, one containing the measured energy values and the other the corresponding $(x, y)$-position. Each file represents a certain time interval of the experiment. The duration of the time interval after which these files are written can be adjusted. This enables one to monitor signals as a function of time, which is very valuable in studies of sample damage due to proton bombardment. The monitoring of the data and the data-analysis are discussed below.

The real-time data-acquisition and the experiment control is carried out by PhyDAS (Physics Data Acquisition System). This data-acquisition system consists of a Phybus part and a VME part. Phybus contains the experimental interfaces (ADC's, stepper motor controllers, etc.) needed for the control of the experiment and acquisition of the list mode data. In the Phybus part 3 MCA's (Multi Channel Analyzers) are present that can be used simultaneously to process the events coming from 3 different detectors. One of the main tasks of the VME part of PhyDAS, which contains a Motorolla 68030 microprocessor, is to control the measurement and to take care of the transport of the data acquired by the Phylbus part to a DEC/ALPHA-AXP workstation. This workstation is used for data storage on a 1 Gbyte disk, data monitoring and off-line analysis.

\section{Data monitoring and data analysis}

During acquisition the data can be monitored on-line making use of the data monitoring program COLUMBUS [ZWIJ93, MUT95]. This program contains a database in which all relevant experimental information, such as the detector configuration, amplifier parameters and name and date of experiments are stored. The database also contains the parameters that define the graphical representation of the acquired data. In general two types of graphics are used, namely (i.) the energy spectrum of the sum of a number of points in a preselected part of the area hit by the scanning proton beam or (ii.) a spatial distribution of the number of X-rays (PIXE), protons (NBS/NFS and STIM) or electrons (secondary electron detection) detected in a predefined energy interval within the energy spectrum. The intensity of number of detected $\mathrm{X}$-rays or particles is expressed in either a black and white scale or full colour scale 


\section{Data-analysis during STMM measurements}

As described in Section 2.2, the STTM technique can be employed to determine the local areal mass thickness by measurement of the energy loss of the protons after passage through the specimen, since for each position in the scan pattern a STIM spectrum is obtained. There are different ways to calculate the local areal mass thickness from a STIM energy loss spectrum. In addition to the classical methods of mean-energy filtering and median filtering in this thesis Chauvenet filtering is applied.

a

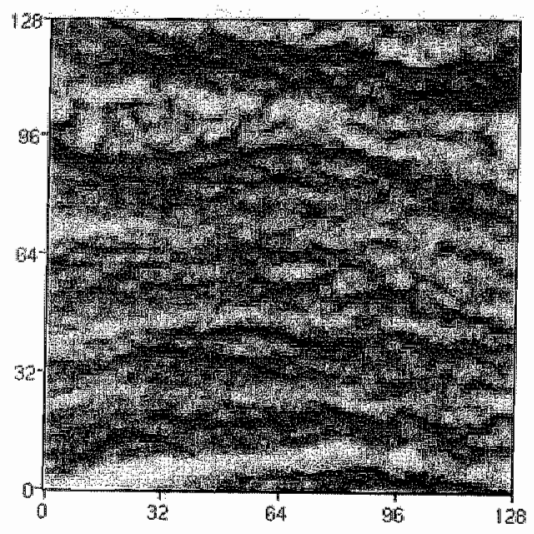

b

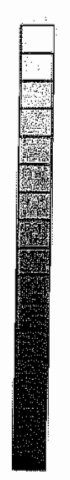

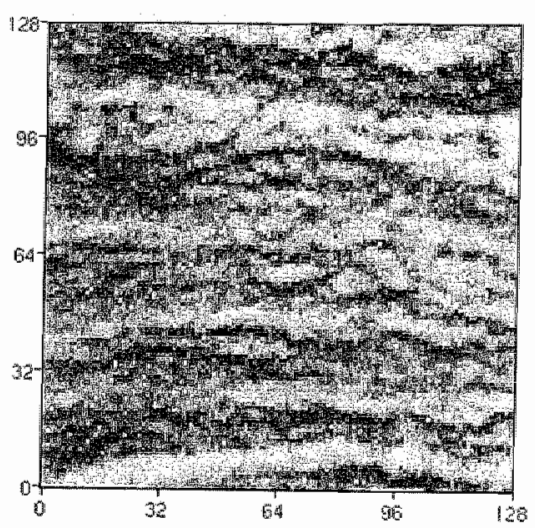

\section{C}
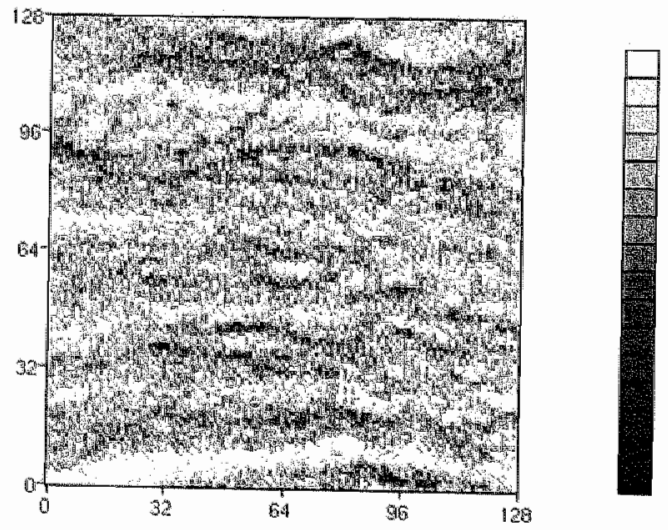

Figure 4. 15: Chawenet (a), median (b) and mean-energy (c) filtered dara from STIM analysis of biological tissue. Note the improvement in noise reduction in case Chauvenet filtering is applied. For this reason this method was used for fitther analysis of STM data. 
The disadvantage of using an average value of the measured energies is the influence of unlikely or spurious events on this value. The influence of these events on the "true" proton energy after passage through the specimen is reduced in case the median value from the measured energies is selected, as was already shown in 1983 by Overley et al. [OVE83]. However, pile-up events will tend to shift the median value to higher energy, whereas low energy tailing (due to slit scattering) has the opposite effect. To find the most probable energy value, it is even more profitable to apply the criterion of Chauvenet [CHA08]. First the mean energy value $\mu$ and the standard deviation $\sigma$ are determined for each pixel scanned by the beam. Subsequently all energy values that deviate more than 1280 from $\mu$ are removed and $\mu$ and $\sigma$ are determined again. Now protons with energies $E_{p}<|\mu-64 \sigma|$ and $E_{p}>|\mu+64 \sigma|$ are excluded. This procedure is repeated for decreasing energy intervals of $32 \sigma, 16 \sigma, 8 \sigma, 4 \sigma$, respectively, until an energy interval of $2 \sigma$ is reached. The final value of $\mu$ is used to colour a pixel in the STIM image. Figure 4.15 shows the result of both mean-energy, median and Chauvenet filtering of the same set of data acquired during the irradiation of biological samples.

\subsection{Summary and concluding remarks}

On the basis of the considerations of this chapter it can be concluded that the Eindhovet proton microprobe set-up is a potential tool for the elemental analysis of biological samples. Elemental concentrations or contents can be determined with a spatial resolution in the order of $3 \mu \mathrm{m}$. As a result, it becomes possible to study the influence of ischemia and subsequent reperfusion on the elemental concentrations on the cellular level. It was also demonstrated that various techniques can be applied for the precise localization of the individual cells within a specific area scanned by the proton beam. Especially the STIM technique, which can be used in addition to PIXE, can be employed to localize cells within freeze-dried cryosections of rat heart tissue. This offers the possibility to investigate the cell-to-cell variation in elemental concentration in injured cardiac tissue.

In this chapter the experimental aspects of the analysis of biological samples with a proton microprobe have been discussed. The proton probe experiments described in the next chapters are performed making use of the equipment and methods as described in this chapter, unless stated otherwise. In the next chapter the preliminary results of the proton probe analysis of normoxic, ischemic and reperfused rat hearts will be presented. However, if physiological meaningful results have to be obtained, it is of utmost importance that the analyzed sample is a true representation of the rat heart in situ and no changes in elemental concentration are introduced during the sample preparation procedures. For this reason in the next chapter special attention is also paid to the possible artefacts introduced during the preparative steps. 
Chapter 4 


\section{Chapter 5}

\section{PIXE microanalysis of elements of normoxic, ischemic and reperfused rat hearts; preliminary results}

\subsection{Introduction}

In the living cell physico-chemical processes are operative to maintain a difference in intra- and extracellular ion concentrations. To this end specific proteins, serving as ion pumps, are present in the cellular membrane. The ion concentration gradients are eliminated due to passive diffusion through the cellular membrane when the membrane pumps stop functioning as a result of damaging conditions, such as ischemia and subsequent reperfusion.

The aim of the study presented in this chapter is to perform X-ray microanalysis on freezedried cryosections of normoxic, ischemic and reperfused heart tissue, using the proton microprobe to determine the changes in cellular content of relevant electrolytes, such as $\mathrm{Na}$, $\mathrm{Mg}, \mathrm{K}$ and $\mathrm{Ca}$. Also some other trace elements, such as $\mathrm{P}, \mathrm{S}$ and $\mathrm{Fe}$, have been determined. In this chapter the preliminary results of PIXE microanalysis of damaged myocardium are shown.

It should be noted that in case the ion content of cells is studied, it is of utmost importance to prevent the redistribution of diffusable elements during tissue handling and analysis in ordet to preserve the elements in their in vivo state. Generally, alterations in the cellular content in normal and diseased myocardium can only be used as a measure of the damage inflicted upon the heart when the element movement is minimized at every stage of the sample preparation procedure. For this reason, in addition to the determination of the alterations in elemental contents, the objectives of this preliminary study presented in this chapter are:

i. to verify whether the measured values for the elemental contents correspond with values from literature,

ii. to gain insight into possible problems, and specially the occurrence of artefacts introduced during the preparative steps, i.e., the removal of tissue from the heart, freezing, sectioning, transport, etcetera.

In Section 5.1 all preparative steps and their possible influence on the measured elemental contents will be discussed. Subsequently, in Section 5.2 the preliminary results of the proton microprobe analysis of tissue sections from healthy and ischemic / reperfused rat hearts are presented. In the same section the results of electron microscope analysis are shown. In this study this technique has been used to trace and recognize artefacts introduced during the preparation procedures. Finally in Section 5.3 the results are summarized and possible improvements for further analysis of biological tissue are given. 


\subsection{Materials and methods}

\subsubsection{Perfusion experiments and sample preparation}

\section{Isolated heart model}

In this work isolated, ejecting rat hearts were used to study the influence of ischemia and reperfusion on the concentration distribution in the heart. The isolated heart preparation has been described previously by Snoeckx [SNO87]. The isolated heart is perfused using a crystalloid buffer solution containing mineral salts and glucose. This model offers the possibility to control coronary perfusion pressure, temperature, $\mathrm{pH}$ and composition of the perfusion fluid. The use of this model also excludes the influence of neural and humoral factors on the results of the study. However, it is important to note that the influence of the isolated heart model, in particular the use of a buffer solution instead of blood, on the cellular content of elements is largely unknown.

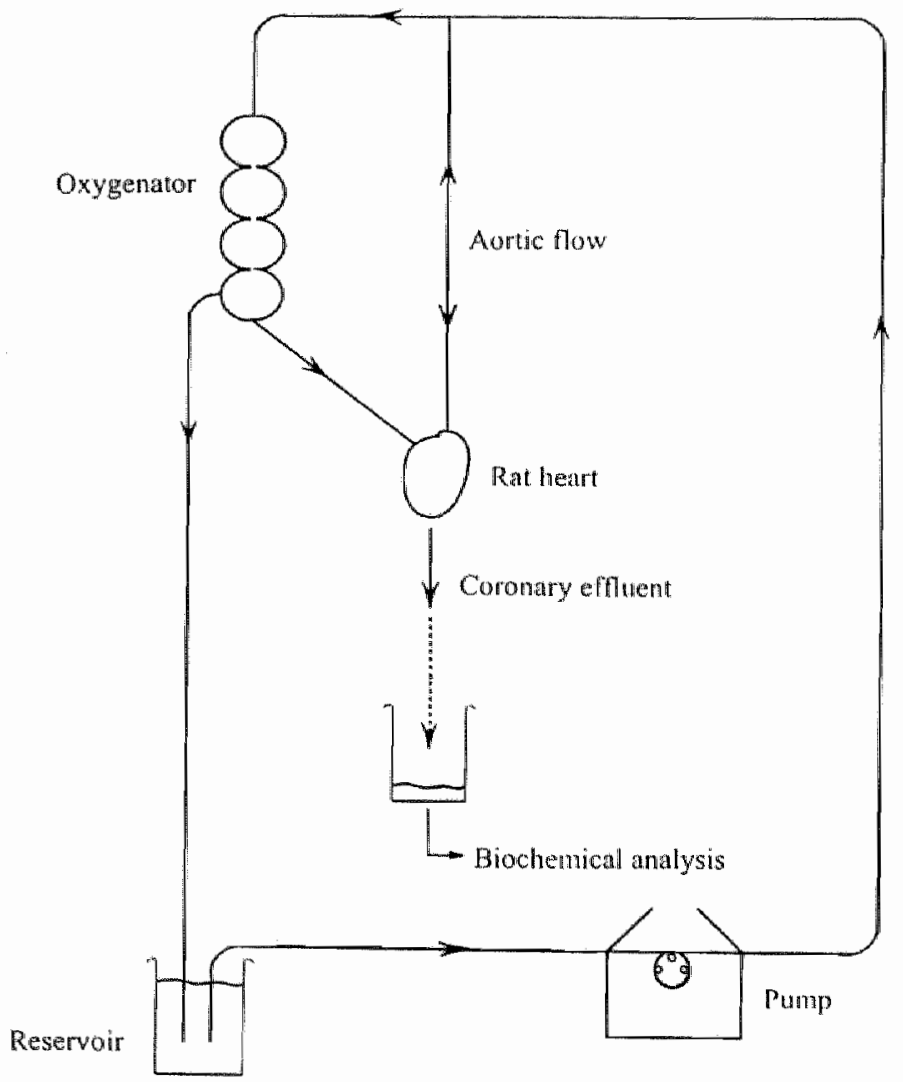

Figure 5.1: Schematic representation of the perfusion apparatus (adapted from [SNOS7]). 
Rat hearts were rapidly removed from angesthetized male Lewis rats (age 13 weeks). Subsequently the isolated hearts were attached to the aortic cannula of the perfusion set-up (see Fig. 5.1). To fill the left ventricle and atrium a second cannula was connected to the left atrium. Upon attachment to the perfusion system within 20 s the hearts started contracting. Generally it took $10-15$ min from the moment of removal of the heart from the rat to the start of heart perfusion in the perfusion apparatus. It is assumed that during this period of ischemia no substantial alterations in the content of the elements of interest were introduced.

The perfusion apparatus is kept in a cage at a constant temperature of $37^{\circ} \mathrm{C}$. The hearts were perfused using a Krebs-Henseleit bicarbonate buffer, which contains (in $\mathrm{mM}$ ): $\mathrm{NaCl}$ (130), $\mathrm{KCl}(5.6), \mathrm{MgCl}_{2}(1.2), \mathrm{CaCl}_{2}(2.2), \mathrm{NaH}_{2} \mathrm{PO}_{4}(1.2), \mathrm{NaHCO}_{3}(28.6)$ and $\mathrm{D}(+)$ glucose (11.1). These values are comparable with the concentration of these components in blood plasma. The pH of the buffer varied between 7.35 and 7.45 . The buffer solution, which is pumped into the system from a reservoir, is continuously gassed with $95 \% \mathrm{O}_{2}$ and $5 \% \mathrm{CO}_{2}$.

All perfusion experiments started with an initial period of 10 min of retrograde perfusion at a pressure of $8 \mathrm{kPa}$ according to the Langendorff technique [LAN1895]. During retrograde perfusion the buffer sollution enters the heart via the aortic cannula, $i, e_{\text {, }}$ in reverse direction (see Fig. 5.2a). As a result the aortic valve is closed and the perfusion medium cannot enter the left ventricle. This means that during the retrograde perfusion period only the coronary arteries are perfused with the buffer solution. Since no fluid is ejected from the left ventricle the heart can stabilize during this period. Subsequently, hearts were subjected to antegrade perfusion (see Fig. 5.2b). In this case the perfusion medium is allowed to enter the left atrium of the heart. After subsequent filling of the left ventricle now the heart starts to eject fluid. The hearts were allowed to eject the buffer solution during $30 \mathrm{~min}$ (pre-ischemic or normoxic period) before the hearts were completely deprived from flow (no-flow ischemia). After 45 min of ischemia flow was re-introduced (reperfusion) during a period of $40 \mathrm{~min}$ (10 min retrograde followed by $30 \mathrm{~min}$ of antegrade perfusion).

During the perfusion experiments the heart rate was kept constant at a frequency of 300 beats: $\min ^{-1}$. To this end two platinum electrodes were attached to the right atrium for external bipolar pacing. Coronary flow was measured by collecting the total amount of coronary effluent during a period of one minute. To monitor cardiac output the coronary flow should be added to the aortic flow, which is measured using an electromagnetic flow probe mounted in the aortic cannula. This enables one to compare the cardiac output during the pre-ischemic period and the reperfusion period. In addition the coronary effluent samples can be used to determine the activities of intracellular enzymes. Since these enzymes cannot leave viable cells, the presence of intracellular enzymes in the perfusate can be used as an indicator of cell damage [LAD54].

\section{Sampling of biopsies, cryofixation and sectioning of myocardial tissue}

Transmural biopsies of the left ventricular wall were taken with an electrically driven , hollow drill ( $1.5 \mathrm{~mm}$ ) at the end of the pre-ischemic (normoxic), ischemic or reperfusion periods. To check for the possible effects of the perfusion with a crystalloid buffer control myocardial biopsies were also taken from the heart in sim in anaesthetized rats immediately after opening. 

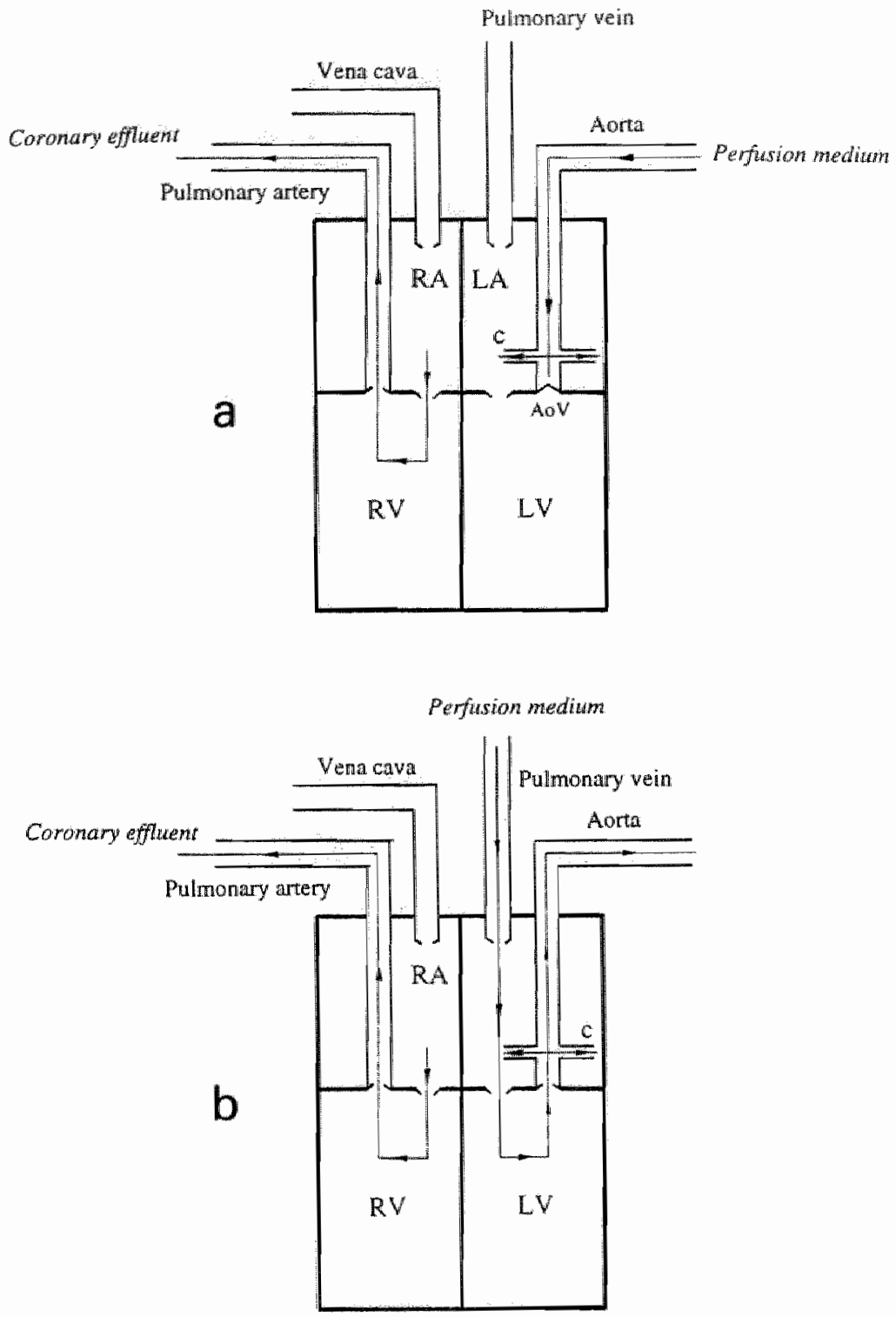

Figure 5.2: Schemanic representation of retrograde $(a)$ and antegrade perfision $(b)$. RA $=$ right atrium: $L A=$ left atrinm: $R V^{\prime}=$ right ventricle; $L V=$ left ventricle; $c=$ coronary arteries. Wia the coronary arteries the blood reaches the right atrium. 
of the chest. After excision the biopsies are removed rapidly from the hollow drill. During the removal of the biopsy from the drill no attempts were made to distinguish the subendocardial (inner) side of the transmural biopsy from the subepicardial (outer) side. This is important to note, since it is known that the subendocardial side is more sensitive to the ischemic insult from which follows that transmural heterogeneity in elemental content may occur.

Since different methods are used to support the specimens in the proton microprobe and the electron microscope further handling of the biopsies is dependent on the analytical technique to be used. For use in the proton microprobe relatively large biopsies with a volume of 1$2 \mathrm{~mm}^{3}$ are mounted on a piece of cork using Tissue-tek (Miles, Elkhart, USA). The biopsies were very rapidly submersed in liquid isopentane chilled by liquid nitrogen $(T=77 \mathrm{~K}$ ). Subsequently, cryosections were cut at $-20{ }^{\circ} \mathrm{C}$ at a thickness of $10-15 \mu \mathrm{m}$ in a cryostat microtome (Anglia Scientific AS600, Cambridge, England). Although the proton beam suffers only a slight broadening while traversing the specimen and thus maintains the same spatial resolution for tens of micrometers of penetration, no thicker sections are used in order to avoid overlap of neighbouring cells (thickness $\sim 10 \mu \mathrm{m}$ ) as much as possible. On the other hand, the use of thinner sections will result in longer measurement times because of the smaller mass traversed by the proton beam. For electron microscopy, biopsies were cut into smaller pieces and were mounted on silver pins using Tissue-tek . The biopsies were frozen in melting ethane and were cut at a thickness of 100-150 nm using an ultra cryo-microtome (LKB cryonova ${ }_{x}$ Bromma, Sweden). To avoid the possible introduction of changes in elemental contents or contaminating elements no cryoprotectants were used in both cryofixation procedures.

It should be noted that upon removal from the ventricular wall the biopsies were still very bulky. For this reason the biopsies were cut into smaller pieces using a surgical blade. During this procedure it can be seen that an appreciable amount of tissue fluid was lost. This fluid most likely represents fluid originally present in the extracellular compartment. It is assumed that this loss of fluid has no effect on the intracellular elemental concentrations, especially when cells from the core of the biopsy are analyzed.

\section{Storage of biopsies}

For use in the proton microprobe the biopsies were stored at $-70{ }^{\circ} \mathrm{C}$ prior to sectioning, whereas for electron microscopic purposes the biopsies were stored under liquid nitrogen. If possible the cryosections were cut on the same day the perfusion experiments were performed. However, in some cases biopsies that had been stored for several months were used. It appeared that the prolonged storage of biopsies at $-70^{\circ} \mathrm{C}$ had a substantial influence on the elementall contents. Proton microprobe analysis of two freeze-dried cryosections, one cut from a biopsy directly after cryofixation and the other cut from the same biopsy after storage for about 5 months, revealed that for some elements, such as $\mathrm{Na}$ and $\mathrm{Cl}$, the tissue content had increased twofold. Since in both cases the tissue specimens were analyzed in a dried state, it is highly unlikely that this difference can be attributed to further drying of the biopsy during prolonged storage at $-70^{\circ} \mathrm{C}$. Up to now no plausible explanation for this effect has been found. Nevertheless, these results show that analysis of samples after prolonged storage should be mistrusted. Therefore in this thesis only the results of proton probe analysis of 
freshly cut samples are taken into account. In contrast with proton probe analysis no attempts were made to study the possible effect of prolonged storage under liquid nitrogen on the elemental content as assessed with the electron microscope.

\section{Preparanion of Formwar backing foils}

Generally, the tissue sections are collected on a backing foil in order to allow analysis in the proton microprobe. For minimizing bremsstrahlung from the support film this backing foil should be as thin as possible and should only contain elements of low atomic number, as follows from $\mathbb{E}$ q. 2.27. In addition, for obvious reasons it should be free of any traces of the elements to be analyzed in the biological material. A comparison of the trace element contamination, bremsstrahlung height at $5 \mathrm{keV}$, and thickness of commercial polymer foils is given by Russell et al. [RUS8 I]. From their study it can be concluded that the lowest possible background is to be obtained from very thin polymer films such as Formvar, which have to be prepared by the user.

For reasons mentioned above, in our study only Formvar backing foils are used for proton microprobe analysis as wells as for electron microscopy. The preparation of Formvar backing foils for use in our proton microprobe was described earlier by [KU191] and more recently by [MUT95]. The methods used by both authors are based upon the immersion of a clean microscope glass in a $2 \%$ (w/v) Formvar (Merck, Darmstadt, Germany) solution, after which the microscope glass is pulled out at a constant speed to obtain a thin Formvar film of homogeneous thickness on the glass surface. After drying in air the Formvar film is removed from the microscope glass by floating of the film at the surface of distilled water, allowing the subsequent pick up of the floating film on a plastic frame.

In the present chapter chloroform was used as a solvent for the preparation of the Formvar foils. PIXE analysis of these Formvar foils revealed a significant $\mathrm{Cl}$ contamination, probably due to use of the chloroform. For this reason in this chapter no chlorine values are given for the hearts analyzed. It appeared that the $\mathrm{Cl}$ contamination was absent in case the Formvar was dissolved in cyclohexanon. Therefore later on in this study (see Chapters 6 and 7) backing foils that were prepared using cyclohexanon are used to pick up sections. In practice it was found more difficult to remove the Formvar film from its glass substrate in case cyclo-hexanon instead of chloroform was used. To ensure an easy removal of the Formwar film it is recommended to allow the film to dry for at least 30 min when prepared with cyclohexanon.

\section{Collection and freeze-drying of issue sections}

For use in the proton microprobe, after sectioning the cold tissue sections were collected on thin Formvar foils covering a $10 \mathrm{~mm}$ hole in a plastic sheet. Although cold sections attach much better to foils kept at room temperature, cold Formvar foils $\left(-20^{\circ} \mathrm{C}\right)$ were used to pick up the sections to prevent thawing of the tissue and possible redistribution of diffusable elements. A threefold increase in the content of both $\mathrm{Na}$ and $\mathrm{Cl}$ was observed when warm Formvar foils were used instead of cold Formvar foils (data not shown). If necessary a second very thin film can be used to cover the tissue sections in order to obtain a better attachment of the tissue section to the backing foil and to prevent curling up of the sections during freeze- 
drying. In order to freeze dry, the samples were kept under vacuum in a dessicator at a temperature of $-20^{\circ} \mathrm{C}$.

For electron microscopy ultrathin $(100-150 \mathrm{~nm})$ sections were collected on thin Formvar films mounted on 200 mesh copper grids using an eye-lash probe and were pressed onto the support film with a polished rod. The sections were subsequently allowed to freeze-dry in a precooled $(\mathrm{T}=77 \mathrm{~K})$ dessicator at a pressure of about $1 \mathrm{~Pa}$ [FRE82].

\section{Transportation of samples}

Since animal experiments and subsequent sample preparation were performed at a different location than the PIXE analysis, freeze-dried cryosections had to be transported to the proton microprobe setup. During transportation, which took about $2 \mathrm{~h}$, in the study presented in this chapter no specific measures were taken to prevent rehydration of the samples, i.e., the samples were transported in a box at ambient temperature and pressure. Following transportation the samples were inserted immediately into the target chamber. Subsequently the target chamber was evacuated. In case the target chamber of the proton microprobe set-up was used for other experiments upon arrival, the samples were stored over silica-gel under ambient pressure in a closed box prior to insertion into the target chamber.

In contrast with proton probe analysis the freeze-dried cryosections could be inserted into the electron microscope immediately after freeze-drying without an interjacent transportation period. However, to check for the possible influence of transportation on the preservation of the cryosections used in the proton microprobe some ultrathin sections were also transported for $2 \mathrm{~h}$ under the same conditions prior to analysis in the electron microscope.

\subsubsection{Proton and electron probe analysis}

In the previous chapter an extensive specification of both the Eindhoven proton microprobe set-up and the CM-12 electron microscope has been given. Therefore in this section only those aspects will be treated that are of specific importance to the results presented in this chapter.

\section{Proton microprobe analysis}

All freeze-dried samples were analyzed using a scanning proton beam with a diameter of about $3 \mu \mathrm{m}$ and a beam current of $100-200 \mathrm{pA}$. The beam was scanned with a $5 \mathrm{kHz}$ scan frequency over an array of $16 \times 16$ pixels, corresponding to an area of about $50 \times 50 \mu \mathrm{m}^{2}$. The beam diameter was considered to give sufficient spatial resolution to measure separate myocardial cells (length $50-100 \mu \mathrm{m}$, thickness $10-20 \mu \mathrm{m}$ ). The total deposited charge waried between 0.5 and $2.5 \mu \mathrm{C}$, resulting in an accumulated charge of $3.9 \mathrm{nC}$ per pixel.

During the PIXE experiments described in this chapter the ultra-LEGe detector instead of the $\mathrm{Si}(\mathrm{Li})$ detector was used for the detection of characteristic X-rays. At the time of the measurements this seemed an obvious choice for a number of reasons. Due to the lower energy needed to create an electron-hole pair in Ge the energy resolution of the LEGe detector compares favourably to our $30 \mathrm{~mm}^{2} \mathrm{Si}(\mathrm{Li})$ detector, making it more suitable for the detection of light elements $(\mathrm{Na}, \mathrm{Mg})$. In addition the Jarger crystal surface area $\left(50 \mathrm{~mm}^{2}\right)$ of 
the LEGe detector should provide a larger solid angle of detection and thus a shorter measurement time. However, later on it became clear that the specifications of this detector were not met in practice In the first place it was shown that due to a faulty construction of the ultra-LEGe detector the maxtmum solid angle of detection can be obtained with the $\mathrm{Si}(\mathrm{Li})$ instead of the ultra-LEGe detector (see Section 4.1). Secondly an inquiry on the specific properties of the LEGe detector elucidated the existence of an extra absorber between the Be entrance window and the detector crystal. This absorber consists of a thin plastic foil with an evaporated aluminum layer, which acts as an infrared shielding. As a result the detector efficiency decreases significantly, specially for X-rays of the light elements, such as $\mathrm{Na}$ and $\mathrm{Mg}$. For this reason all values for the elemental content given in this chapter have been corrected afterwards for this efficiency loss using the manufacturer's values for the thickness of plastic foil and aluminum layer.

\section{Electron microscope analysis}

The histological analysis of ultrathin freeze-dried cryosections was performed using a Philips CM-12 electron microscope. The microscope was operated in the scanning transmission mode at $120 \mathrm{kV}$ and all measurements were carried out with the specimen at ambient room temperature.

\subsection{Results and discussion}

\subsubsection{Electron microscope results}

\section{Preservation of tissue during cryofixation}

In Fig. 5.3 the result of electron microscope analysis of an ultrathin freeze-dried cryosection of control tissue is shown. The analyzed specimen was transferred into the electron microscope directly after freeze-frying and subsequent carbon coating, which precludes the possibility of artefacts due to transportation.

In agreement with earlier results from studies of Zierold [ZIE80], Frederik [FRE81] and Dempsey and Bullivant [DEM76] the figure shows that satisfactory preservation of the fine structure is achieved only in the outer region $(20-30 \mu \mathrm{m})$ of the specimen. Further below the surface of the specimen ice-crystal formation deteriorates the preservation of the ultrastructure of the cells and may result in mechanical rupture of cell membranes. The formation of icecrystals is mainly due to the fact that the time necessary to cool a location at a certain depth from the surface is greatly influenced by the low thermal conductivity of ice. Especially for relatively large samples ( $>0.1 \mathrm{~mm}$ in all directions) it is not the exchange of heat between the specimen and the coolant, but the heat transport inside the specimen itself that becomes important. This means that the cooling of the core of such a specimen is limited by the heat transfer through the already cooled outer layers of the sample. It thus can be concluded that for relatively large samples the choice of the coolant, i.e, liquid isopentane chilled by liquid nitrogen and melting ethane, is not critical. As a result fast submersion of the tissue specimen 
in a liquid coolant (plunge-freezing) can be employed to obtain samples that are appropriate for X-ray microanalysis.

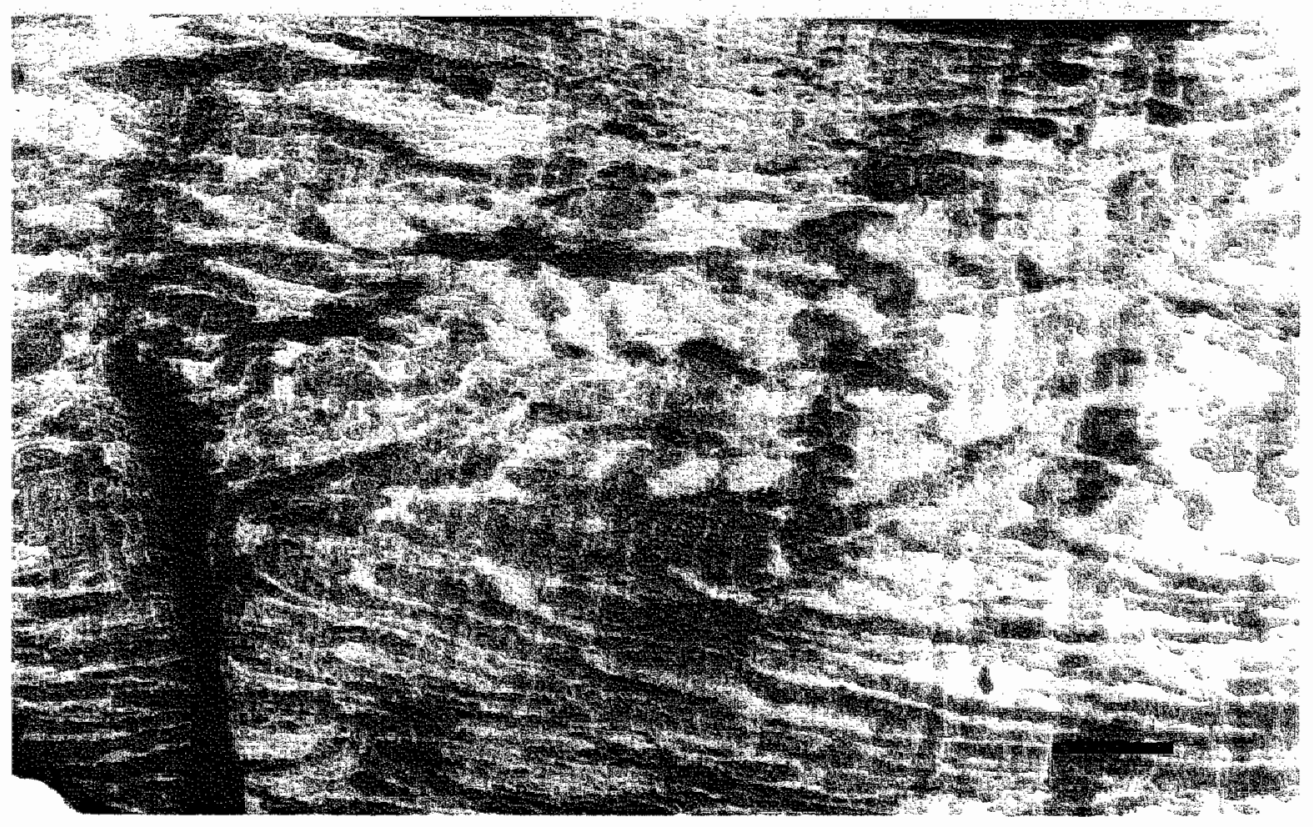

Figure 5.3. Electron microscope analysis of an witrathin freeze-dried cryosection from rat heart. Bars represent $1, \mu \mathrm{m}$.

It should be noted that the formation of ice-crystals is of minor importance in case the dimensions of the ice crystals are smaller than the spatial resolution of the measuring technique employed. It can therefore be concluded that also the inner region of the cryosection can be used for X-ray microanalysis using the proton beam as employed in PIXE.

\section{Artefacts introduced during removal of tissue from the ventricular wall}

Comparison of electron microscope pictures from the surface (a few cell layers) and the inner region of cryosections obtained from normal cardiac tissue showed that in some areas the mitochondria in the surface regions contained dense granules (Fig. 5.4). Electron probe microanalysis of these mitochondria revealed high $\mathrm{Ca}$ and $\mathrm{P}$ levels. This accumulation is probably induced during excision of the biopsy from the heart prior to cooling, causing membrane damage and cell injury at the surface of the tissue specimen at these preparative steps. In the inner region no $\mathrm{Ca}$ accumulation was found in the mitochondria. Therefore the conclusion can be drawn that it is important to check cryosections in the electron microscope prior to proton microprobe analysis to discriminate between effects of sample preparation and 
the effects of (patho)physiological conditions on the tissue content of elements. Moreover it is advisable not to use the outer region of the samples for X-ray microanalysis.

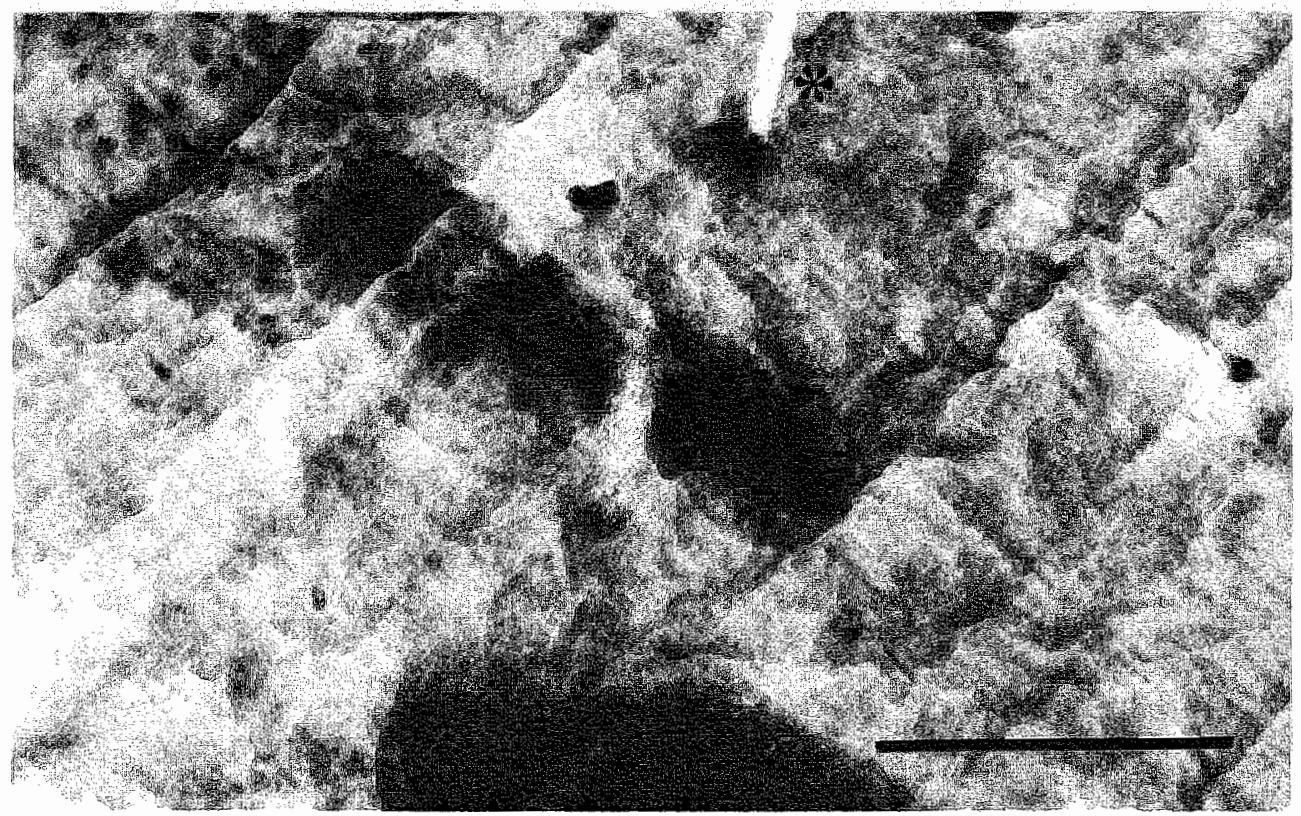

Fig. 5.4: Electron microscope picture of the outer part of the visswe sections. Dense granules are visible in the mitochondria. Bar represents I 4 m. Asterisk indicates sample damage caused by the scanning electron beam used for X-roy microanalysis.

\section{Infinence of transportation on sample preservation}

To investigate whether the morphology of the freeze-dried cryosections remains unaltered during transportation an ultrathin section was analyzed in the electron microscope before and after transportation to the proton microprobe setup. It can be seen from Fig. 5.5 that after transportation, lasting about two hours, black stains are visible in the electron microscope pictures. According to Frederik and Busing [FRE81] these stains probably are the result of rehydration.

It has been demonstrated by Von Zglinicki and Uhrik [ZGL88] that slight rehydration has only little effect on the elemental distribution of carbon coated cryosections. However, since the samples to be analyzed in the proton microprobe are not coated with a protective carbon layer special attention should be paid to this problem in view of redistribution of diffusible elements. In our case coating of the samples with a thin carbon layer prior to proton probe analysis has proven to be a less appropriate option, since the samples would be contaminated with elements present in the oil of the vacuum pump of the carbon coating apparatus (data not shown). 

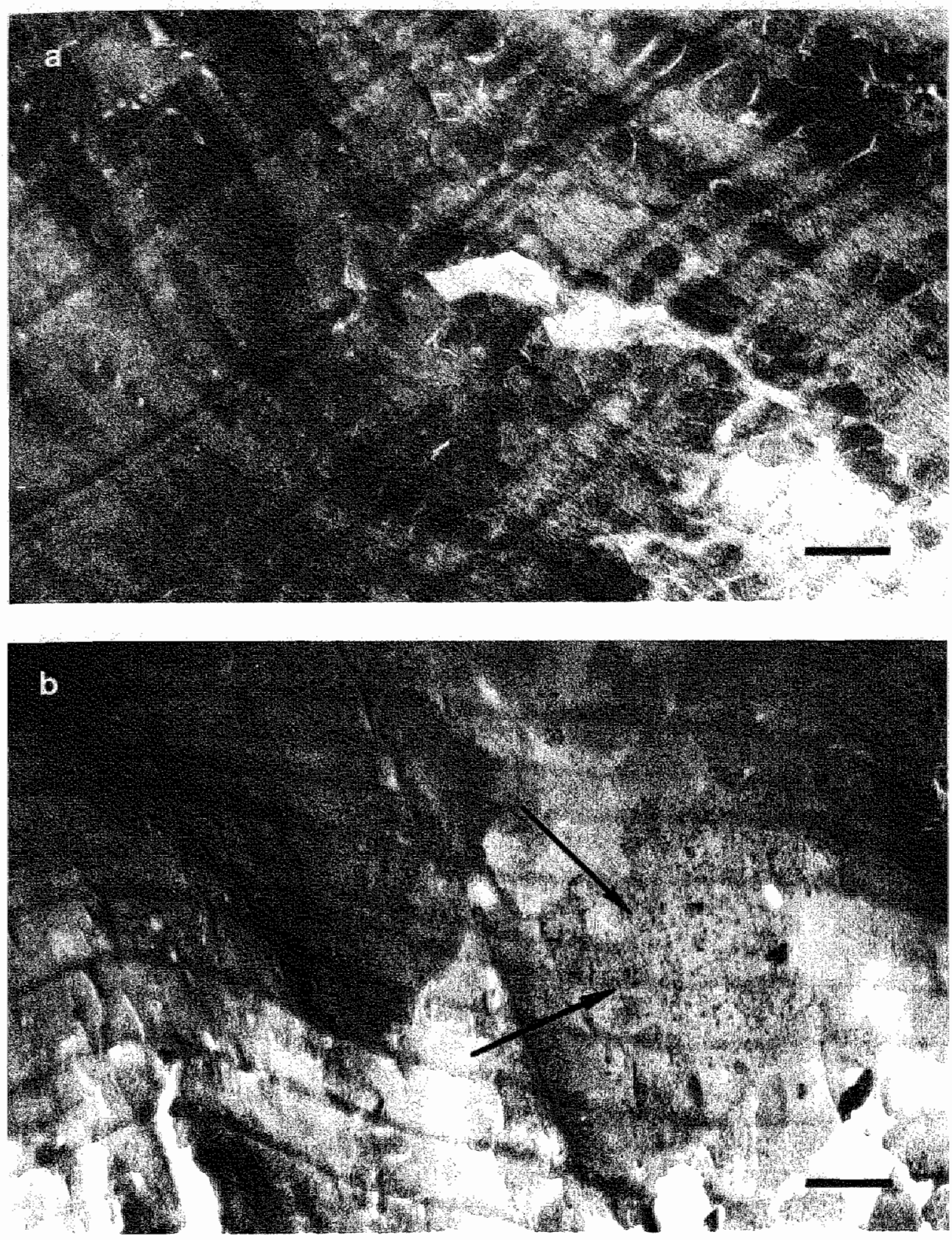

Figure 5.5: Electron microscope pichure of am whathin cryosection before (a.) and after transpartation (b.), respecrively. Transportation lasted about two hours at ambient temperature and pressure, which is identical with the canditions under which the proton probe samples are transported. Dark electron opaque grantules (indicared by arrowsy, probably due to rehydration, are wisible. Bar represents $l \mu \mathrm{m}$. 


\subsubsection{Preliminary proton microprobe results}

The mean elemental content for $\mathrm{Na}, \mathrm{Mg}, \mathrm{P}, \mathrm{S}, \mathrm{K}, \mathrm{Ca}$ and $\mathrm{Fe}$ is displayed in Table $5.1 \mathrm{fi}$ control, normoxic, ischemic and reperfused hearts. Although the number of analyzed sampli is limited, some tendencies became manifest (see below):

Table 5. I: Mean content of electrolytes \pm S.D. for control, normoxic (pre-ischemic), ischemic ar reperfused rat heart. Contents are expressed in $\mathrm{mg} / \mathrm{kg}$ dry weight (ppm). It should $\mathrm{l}$ noticed that for every single phase (control, normoxia, ischemia and reperfusion abour three biopsies were taken from one or two rat hearts. From these biopsies 4 . cryosections were analyzed for every phase.

\begin{tabular}{|c|c|c|c|c|}
\hline \multirow[b]{3}{*}{ Ellement } & \multicolumn{4}{|c|}{ Perfusion condition } \\
\hline & \multirow{2}{*}{$\begin{array}{l}\text { "in vivo" } \\
\text { controls }\end{array}$} & \multicolumn{3}{|c|}{ "in vitro" } \\
\hline & & $\begin{array}{c}\text { pre-ischemic } \\
\text { perfusion } \\
\text { (normoxia) }\end{array}$ & $\begin{array}{l}\text { no-flow } \\
\text { ischemia }\end{array}$ & $\begin{array}{c}\text { post-ischemic } \\
\text { perfusion } \\
\text { (reperfusion) }\end{array}$ \\
\hline $\mathrm{Na}$ & $1,100 \pm 390$ & $2,500 \pm 360$ & $2,000 \pm 720$ & $2,900 \pm 390$ \\
\hline $\mathrm{Mg}$ & $620 \pm 310$ & $620 \pm 160$ & $480 \pm 240$ & $430 \pm 190$ \\
\hline $\mathrm{P}$ & $16,000 \pm 2,100$ & $15,500 \pm 500$ & $13,500 \pm 2,700$ & $14,000 \pm 1,600$ \\
\hline $\mathrm{S}$ & $12,500 \pm 2,300$ & $12,000 \pm 750$ & $12,500 \pm 2,700$ & $10,000 \pm 1,400$ \\
\hline $\mathbb{K}$ & $12,500 \pm 1,500$ & $9,200 \pm 2,000$ & $10,000 \pm 1,500$ & $8,200 \pm 960$ \\
\hline $\mathrm{Ca}$ & $150 \pm 30$ & $220 \pm 120$ & $110 \pm 20$ & $630 \pm 530$ \\
\hline $\mathrm{Fe}$ & $320 \pm 80$ & $230 \pm 20$ & $190 \pm 50$ & $200 \pm 30$ \\
\hline
\end{tabular}

\section{Conirol phase}

In order to verify whether the measured values for the elemental content in the control tissu sections, i.e., sections prepared from cardiac biopsies obtained from hearts in sitw, corresponc with values from literature, the dry weight fractions have to be converted into mmol/kg dr! weight or into wet weight concentrations ( $\mathrm{mmol} / \mathrm{l})$. Wet weight concentrations were estimatec from dry weight contents assuming a dry weight fraction of 0.31 . This value was obtained by taking the mean value of several dry weight fractions published by different authors [SAS94 WEN91, SPE80]. The resulting values for the elemental content are given in Table 5.2. Fol comparison in the same table the concentrations found by other authors for several types 0 : tissue are included. 
If the values for the elemental content obtatned during our study are compared with values from literature it appears that the $\mathrm{Na}$ levels are substantially higher and the $\mathrm{K}$ levels are somewhat lower. There are several possible explanations for this effect:

i. due to rehydration of the freeze-dried tissue sections during transportation the concentration of diffusable elements that are permeable through the cell membrane may change.

ii. since the mean values of an area of about $50 \times 50 \mu \mathrm{m}^{2}$ scanned by the proton beam are given in the table, the possibility exists that these values represent mean of elemental content of the extracellular space and that of the intracellular compartment.

Table 5.2 Comparison of the proton microprobe results of control cardiac issue with data from literature. The references, topes of tisste analyzed, animal species and the analytical techniques wsed are given. The concentravions are expressed in mmolkg dry weight. NMR refers to Nuclear Magneric Resonance spectroscopy el. micr. refers to Electron Probe MicroAnalysis.

\begin{tabular}{c|c|cccccc}
\hline \multirow{3}{*}{ element } & authors & SAS94 & KIR89 & MIL95 & THA92 & HAG90 & WEN91 \\
\cline { 2 - 8 } & species & human & rat & ferret & rat & rat & rat \\
\cline { 2 - 8 } & technique & el. micr. & N.M.R. & el. micr. & el. micr. & el. micr. & el. micr. \\
\hline $\mathrm{Na}$ & 32 & & 84 & 64 & 30 & 43 \\
$\mathrm{Mg}$ & & 40 & 41 & 45 & & 36 \\
$\mathrm{P}$ & & & & 353 & 453 & & 339 \\
$\mathrm{~S}$ & 97 & & 80 & 232 & 80 & 152 \\
$\mathrm{Cl}$ & 348 & & 437 & 643 & 391 & 444 \\
$\mathrm{~K}$ & 2.6 & & 2.49 & 6 & n.d. & 2.6 \\
$\mathrm{Ca}$ & & & & & 208 & & 352 \\
\hline
\end{tabular}

\section{Normoxic phase}

At the end of the pre-ischemic perfusion period (normoxia) the $\mathrm{Na}$ level was found to be increased twofold as compared to control tissue. The normoxic hearts show a distinctive decrease in $\mathrm{K}$ content. $\mathrm{Ca}$ levels are still below about $500 \mathrm{ppm}$. In addition a decrease in $\mathrm{Fe}$ content was observed after perfusion with a crystalloid buffer. Although the decline in $\mathrm{Fe}$ is not completely understood, the removal of red blood cells by the crystalloid buffer contributes to this phenomenom. 
Since no dechne in functional stability of the heart was observed during the perfusion. experiments, the alterations in $\mathrm{Na}$ and $\mathrm{K}$ contents most probably reflect an adaptation of the hearts to the perfusion with the crystalloid buffer, rather than a first stage of cellular damage: Moreover, it should be noted that the concentrations of the elements present in the crystalloid buffer solution are comparable with the concentration of these elements in blood plasma. For this reason the concentration of some elements in the crystalloid buffer can differ substantially from blood walues, since in blood in addition to the electrolytes in blood plasma also blood: cells with electrolytes are present. In case no distinction can be made between the intra- and extracellular space during proton probe analysis this might be of infuence on the mean elemental content of the total area scanned by the proton beam.

\section{Ischemic phase}

There are no substantial differences between the electrolyte contents after the pre-ischemic and ischemic periods (Table 5,1). There are two possible explanations can be given for this. result. First, this may imply that the ischemic insult in this study was not severe enough to create an appreciable imbalance in the electrolyte homeostasis of the flow-deprived cells. Second, it shoull be noted that during the ischemic period the cells are completely deprived from flow, i.e., no transportation of electrolytes from and towards the cells can take place. Especially if the intra- and extracellular space cannot be discriminated it is likely that no appreciable alterations in elemental content are introduced during the ischemic period

\section{Reperfusion phase}

After re-introduction of flow (reperfusion) substantial alterations in the cellular contents were observed, specially for $\mathrm{Ca}$ and $\mathrm{K}$. The $\mathrm{K}$ levels decrease as compared to ischemic tissue, whereas an appreciable increase in the Ca content is observed. Accumulation of calcium in the reperfused heart is most likely caused by a complex set of events. On the one hand the sarcolemma may become more permeable for extracellular $\mathrm{Ca}^{2 *}$ whereas calcium pumps in the same membrane and in the sarcoplasmatic reticulum may lose their proper function, leading to accumulation of calcium in the cytoplasmic space. An important property of the mitochondria is to sequester $\mathrm{Ca}^{2 *}$ ions to keep the cytoplasmic $\mathrm{Ca}^{2}$ levels low. Therefore it is assumed that part of the $\mathrm{Ca}^{2+}$ is transported into the mitochondral matrix which in turn causes precipitation of calcium salts inside the mitochondrial matrix. The accumulation of sodium in the cell during reperfusion may also be responsible for an additional calcium entry in the cell wia the $\mathrm{Na}^{*} / \mathrm{Ca}^{2 *}$ exchanger, even when the membrane is not irreversibly damaged [KAT92]. Loss of cellular potassium is most likely caused by impairment of the sarcolemmal Na-K-ATPase resulting in enhanced $\mathrm{Na}$ and reduced $\mathrm{K}$ levels inside the cell. As a consequence the electrophysiological properties of the reperfused cells will alter.

Mg loss can occur when the cellular level of high energy phosphates such as ATP, an intracellular $\mathrm{Mg}$ binding component, is substantially declined. As a consequence more free $\mathrm{Mg}$ is present in the cytoplasm and is subsequently released after reperfusion. A decline in cellular Fe most likely reflects the release of myoglobin, a low molecular weight iron-containing protein which is abundantly present in the normoxic cardiac cell. Loss of cellular integrity due 
to ischemia and reperfusion is commonly accompanied by a release of myoglobin into the extracellular space [JEN83].
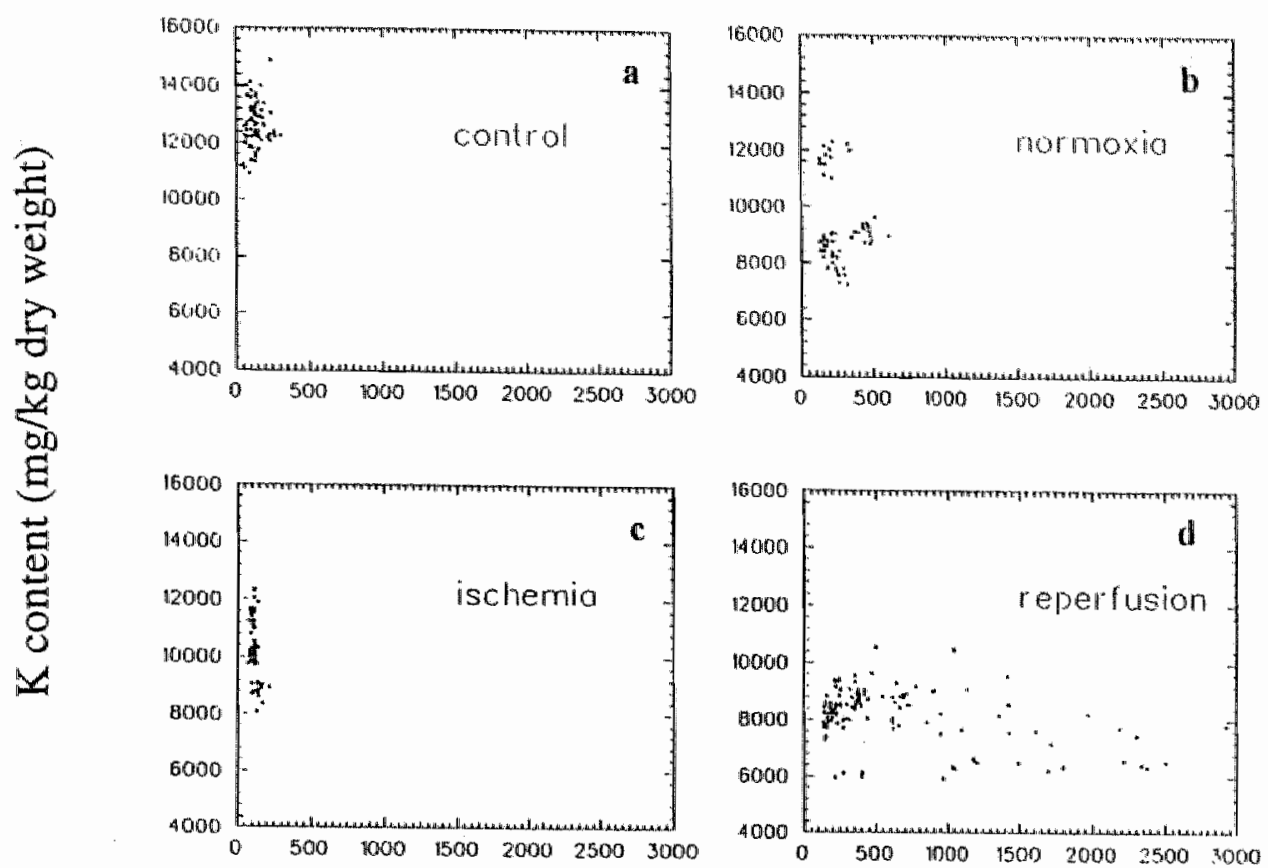

\section{Ca content $(\mathrm{mg} / \mathrm{kg}$ dry weight)}

Figure 5.6: Changes in $K$ and $C a$ content for control, normoxic (pre-ischemic), ischemic and reperfised rat hearts. Each point in the figure represents the inean $K$ and $C a$ content of 16 pixels. since for all measterements $16 \times 16$ pixels $\left(50 \times 50 \mathrm{~mm}^{2}\right)$ were grouped together into 16 parts of $4 \times 4$ pixels (about $12 \times 12 \mu \mathrm{m}^{2}$ ). For every single phase (control, normoxia. ischemia and reperfusion) about three biopsies were taken from one or two rat hearts.

\section{Comparison between the local $\mathrm{Ca}$ and $\mathrm{K}$ content}

Because the alterations in elemental content were most manifest for $C a$ and $K$, the relation between the local $\mathrm{Ca}$ and $\mathrm{K}$ contents is shown in more detail in Fig. 5.6 for all analyzed phases. For all control hearts low $\mathrm{Ca}$ levels and high $\mathrm{K}$ levels are found. From literature [KAT92] it is known that in normal cardiac tissue large ion gradients across the plasma membrane for both $\mathrm{K}$ and $\mathrm{Ca}$ exist with the intracellular $\mathrm{K}$ content higher than that in the extracellular fluid. The opposite is true for $\mathrm{Ca}^{2+}$. However, analysis of the control tissue 
(Fig. 5.6a) revealed little spread in $\mathrm{Ca}$ and $\mathrm{K}$ levels and no large gradients in $\mathrm{Ca}$ and $\mathrm{K}$ contents were observed. Therefore it can be concluded that either only the intracellular space was scanned by the proton beam or mobile electrolytes were redistributed, most likely due to rehydration of the freeze-dried tissue sections, resulting in contents which are mean values of intra- and extracellular values.

A distinctive decrease in $\mathbb{K}$ content in both nomoxic (5.6b) and ischemic hearts $(5.6 \mathrm{c})$ is observed as compared to control tissue (5.6a), whereas Ca levels are still below about 500 ppm. Fügure $5.6 d$ (reperfusion phase) shows a further decrease in $K$ content, as well as a dramatic increase in Ca levels. Calcium contents now are varying from about 150 ppm up to 3,000 ppm dry weight. This implies that locally very high Ca levels are present.

In Fig. 5.6 no information is given about the local distribution of the elements, i.e, whether low and high levels are randomly distributed or that accumulations of electrolytes were found. Local variations in electrolyte levels may become visible by mapping of elemental contents (see Fig. 5.7).
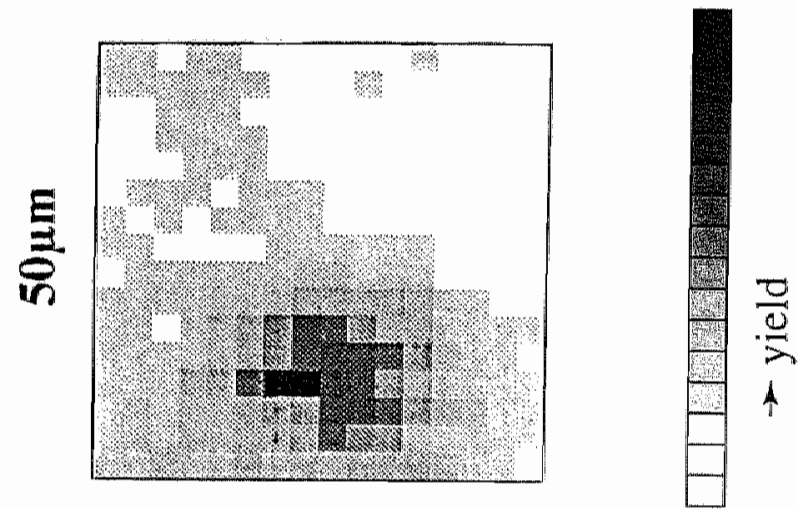

$50 \mu \mathrm{m}$

Figure 5.7: Typical result from PLE microamalysis of the element $C a$ in a freeze-dried cryosection of a reperfised rat heart. Scan dimensions: $50 \times 50 \mathrm{\mu m}^{2}$.

It appeared that for the control hearts and hearts subjected to pre-ischemic perfusion and ischemia the elemental contents were distributed uniformly over the scanned area (figures not shown). In reperfused hearts however, some analyzed samples showed locally very high and varying Ca levels (see Fig. 5.7). This can be explained by the fact that either intercellular differences are present (in case two adjacent cells instead of one are analyzed by the proton microbeam) or that intracellular heterogeneity in $\mathrm{Ca}$ content is present in the reperfused myocardium. The latter can be caused for instance by the precipitation of calcium phosphate crystals in the mitochondrial compartment of the reperfused cardiac cell, which could be indicative of the fact that the local calcium content is a measure of the induced damage in situ. 


\subsection{Summary and concluding remarks}

In conclusion it can be stated that the preliminary results of the $\mathrm{X}$-ray microanalysis of control, normoxic, ischemic and reperfused rat hearts show that proton microprobe analysis is a useful tool in myocardial injury studies. Alterations in $\mathrm{Na}, \mathrm{Mg}, \mathrm{K}$ and $\mathrm{Ca}$ contents can be followed with a spatial resolution in the order of the beam diameter. As alterations in intracellular $\mathrm{Ca}$ and $\mathrm{K}$ levels are most likely indicative of the induced damage, it becomes possible to study the effects of ischemia on a cellular level.

Utmost care is required during the sample preparation procedures, since it was found that a number of artefacts can be easily introduced. Concerning the preparative steps the electron microscope has proven to be very useful in tracing and recognizing artefacts induced during the complete process of sample preparation. In summary a number of pitfalls could be recognized. Firstly the outer region of the samples should not be used for X-ray microanalysis due to the cellular damage at the surface of the tissue specimen, which is probably induced during removal of the biopsy from the ventricular wall. Secondly it appeared that both the use of biopsies that have been stored at $-70^{\circ} \mathrm{C}$ for a long period of time as well as the use of warm backing foils to pick up frozen cryosections result in altered elemental contents. Therefore these two conditions should be avoided during sampling handling. Finally it can be concluded that also the prevention of rehydration of the freeze-dried samples seems to be important if contents which reflect the situation in wivo have to be obtained in PIXE microanalysis. In case freeze-dried samples become rehydrated possible element migration may give rise to contents measured which approach mean values of intra- and extracellular values. For this reason it is advisable to keep the samples under vacuum during transportation. The coating of the samples with a thin carbon layer seems a less appropriate option, due to the contamination with elements present in the oil of the vacuum pump of the carbon coating system.

In the case of $\mathrm{X}$-ray microanalysis using a beam with a diameter of a few $\mu \mathrm{m}$, analysis of cells in a tissue section requires precise localisation of the scanned area, particularly when local differences in elemental content are expected within an analyzed freeze-dried cryosection. To identify cellular structures the combination of PIXE microanalysis with STIM, making use of the better spatial resolution that can be obtained with the latter technique, has to be employed. The use of STIM analysis as a tool to localize cells, combined with the use of stained adjacent tissue sections, will be presented in the next two chapters. 


\section{Chapter 6}

\section{Proton microprobe analysis of rat hearts subjected to calcium paradox experiments}

\subsection{Introduction}

Massive cardiac damage can be introduced by subjecting hearts to the so-called calcium paradox [ZIM66]. The calcium paradox is caused by perfusing isolated hearts for a relatively short period with a $\mathrm{Ca}^{2+}$ free buffer followed by perfusion with a $\mathrm{Ca}^{2+}$ containing buffer. According to Ganote et al. [GAN83] the readmission of $\mathrm{Ca}^{2+}$ results in a large influx of free $\mathrm{Ca}$ ions into the cardiomyocytes, which in turn is most likely responsible for the severe myocardial darnage due to hypercontraction of the myofibrils. The precise mechanism underlying the influx of $\mathrm{Ca}$ ions during the calcium paradox is not fully understood. It has been suggested that alterations in cellular $\mathrm{Na}$ levels provoke the massive influx of $\mathrm{Ca}^{2+}$.

The aim of the study presented in this chapter is to measure the changes in the distribution of elemental contents in hearts subjected to the calcium paradox. This experimental model was chosen to ensure a high level of induced damage, so that substantial changes in the cellular content of elements have to be appreciated. The results of this extreme injury may serve as an indicator of the maximum alterations in the content of calcium and other elements that are to be expected in case the hearts are subjected to less damaging conditions, such as short-term ischemia followed by reperfusion.

The elemental contents were assessed with the proton microprobe technique. Additionally the électron microscope was used to confirm some findings obtained from proton microprobe analysis. Light microscopy, Scanning Transmission Ion Microscopy (STIM) and electron microscopy were used to study the histological and morphological changes following the calcium paradox. In addition enzyme release from the irreversibly damaged hearts was assessed to serve as a measure of the extent of loss of cellular viability.

\subsection{Materials and methods}

\section{Perfission protocol and sample preparation}

Isolated rat hearts from male Lewis rats were used in all experiments. The animals were anaesthetized with Hypnorm $(0.01 \mathrm{mg} / 100 \mathrm{~g}$ body weight fentanyl plus $0.5 \mathrm{mg} / 100 \mathrm{~g}$ body 
weight fluanisone s.c). The hearts were removed from anaesthetized animals after thoracotomy and were subsequently mounted to the aortic cannula of the perfusion system to allow perfusion with a Krebs-Henseleit bicarbonate buffer solution (see also Chapter 5 ). Rat hearts were subjected to both normoxic perfusion $(n=3)$ and the calcium paradox manoeuvre $(n=5)$. The perfusion protocol is shown in Figure 6.1.
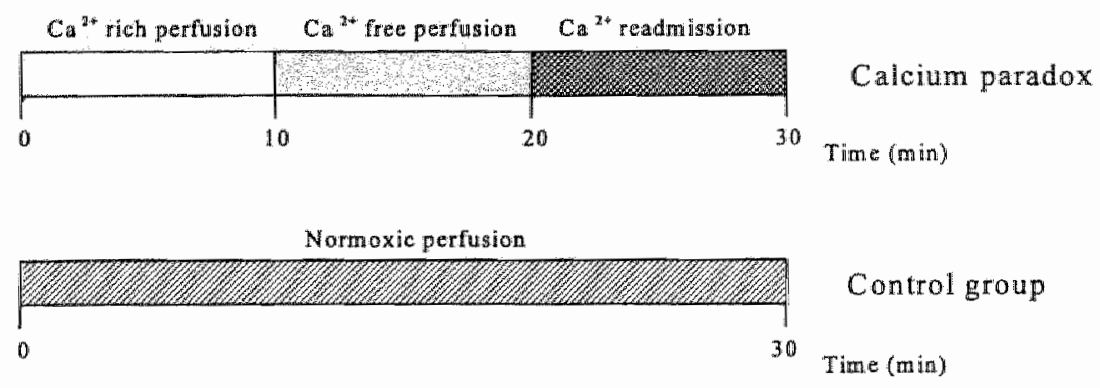

Figure 6.1: Schematic representation of the perfision protocol for calcium paradox experiments and normaxic group. Tisstue biopsies were taken at the end of the $\mathrm{Ca}^{2+}$ readmission period. Effuent samples were taken during 10 min of $\mathrm{Ca}^{2+}$ readmission. The $\mathrm{Ca}^{2+}$ concentration in the $\mathrm{Ca}^{2+}$ containing buffer, which was used durring bath normoxic perfusion and $\mathrm{Ca}^{2+}$ paradox experiments, amounted to $2.2 \mathrm{mM}$. Control tissue biopsies were taken after 30 min of normoxic ( $\mathrm{C} \mathrm{a}^{2+}-$ rich) perfision. Note that the perfission medium used for $\mathrm{Ca}^{2+}$ rich, $\mathrm{Ca}^{24}$ readmission and normoxic perfusion is identical and contains $2.2 \mathrm{mM}$. The medium used for the $\mathrm{Ca}^{2+}$ free perfision was exactly the same, only did not contain $\mathrm{Ca}^{2 *}$.

After $10 \mathrm{~min}$ of control perfusion with a buffer solution containing $2.2 \mathrm{mM} \mathrm{Ca}{ }^{2.2}$, the perfusion buffer was replaced by a $\mathrm{Ca}^{2+}$-free solution. The duration of the $\mathrm{Ca}^{2+}$-free perfusion period lasted $10 \mathrm{~min}$, followed by another $10 \mathrm{~min}$ of perfusion with the control, calcium containing buffer solution. The hearts were perfused retrogradely according to the Langendorff technique during both $\mathrm{Ca}^{2+}$-rich and $\mathrm{Ca}^{2+}$-free perfision. This means that during all perfusion experiments only the heart tissue itself was perfused and no ejection of fluid from the left ventricle took place (see also Chapter 5). No pacing of the hearts was applied.

In this study the $\mathrm{Ca}^{2+}$ free perfusion was chosen to last 10 min in order to induce severe damage in both the endo- and epicardial side of the left ventricular wall and thus to prevent transmural heterogeneity [SLE92]. In order to achieve a calcium paradox, two separate perfusion systems were used (see Fig. 6.2). At the end of the control period the central valve is closed and the other valves at the left side are opened to allow complete removal of the remaining $\mathrm{Ca}^{2+}$ containing buffer. Subsequently, the central valve is opened to start perfusion with the $\mathrm{Ca}^{2+}$ free buffer solution. After 10 min the reverse protocol is executed to allow readmission of $\mathrm{Ca}^{2+}$. 


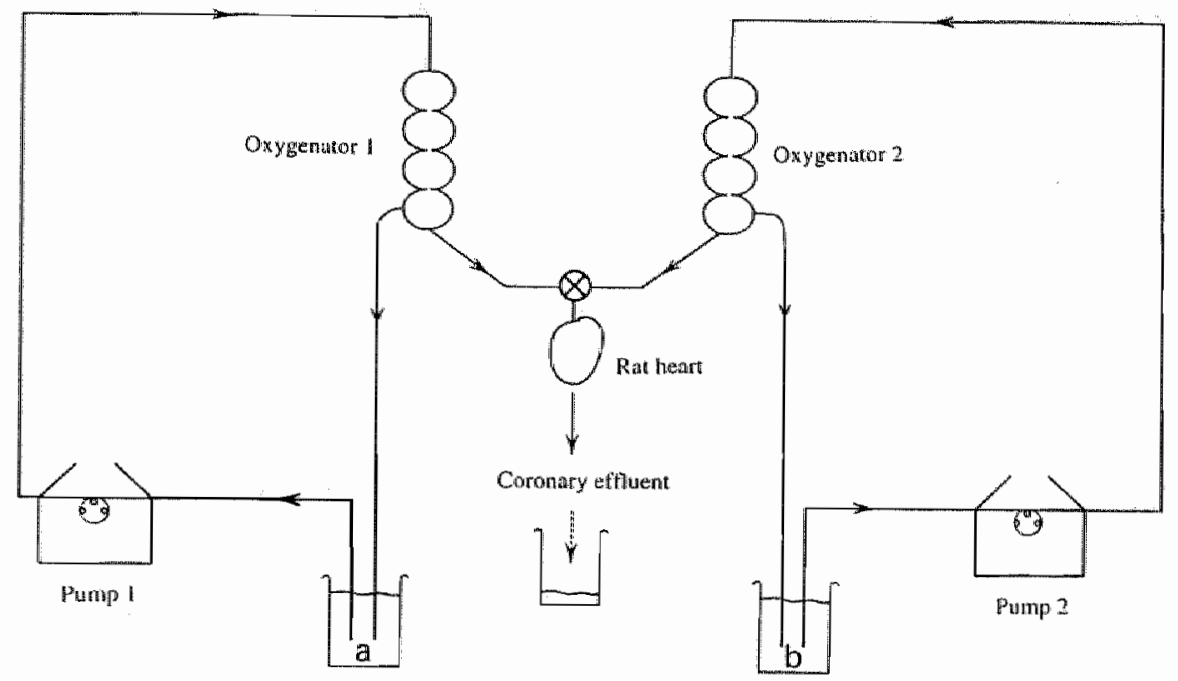

Figure 6.2: Perfusion apparatus used to induce calcium paradox. The two perfusion systems are completely separated to awoid mixing of the $\mathrm{Ca}^{2+}$-free (a) and $\mathrm{Ca}^{2+}$-containing (b) buffer solutions.

\section{Effruent sampling}

During the $\mathrm{Ca}^{2+}$ readmission period coronary effluent was sampled to allow determination of the release of the cytoplasmic enzyme lactate dehydrogenase (LDH). An aliquot of $15 \%$ of bovine serum albumin (BSA) was added to the effluent to prevent loss of the enzymatic activity during storage. The effluent samples were stored at $-80^{\circ} \mathrm{C}$ prior to analysis of LDH. The LDH release was determined spectrophotometrically using a Cobas Bio autoanalyser according to Bergmeyer and Bernt [BER74] and results were normalized on heart weight.

\section{Tissue sampling}

At the end of the $\mathrm{Ca}^{2+}$ readmission period left ventricular biopsies were taken. Control myocardial biopsies were taken from the heart after $30 \mathrm{~min}$ of in vilo normoxic perfusion (see also Section 5.1). For analysis with the proton microprobe the biopsies were plunge-frozen in isopentane chilled by liquid nitrogen. Subsequently sections were cut at a thickness of $10 \mu \mathrm{m}$ in a cryostat microtome and were collected at thin Formvar foils (see Chapter 5). These sections were allowed to freeze-dry in an evacuated dessicator prior to analysis in the proton microprobe. Adjacent sections of the same thickness were collected on microscope slides and were stained with toluidine blue according to the procedure as described in Section 4.2.1. 
Smaller parts of the same biopsies were mounted on silver pins and subsequently frozen in liguid ethane to allow sectioning in a ultra cryomicrotome. For electron microscopy purposes sections were cut at a thickness of $100-150 \mathrm{~nm}$. The cryosections were collected using an eye-last probe on 200 mesh Cu grids covered with Formvar foils. Subsequently the cryosections wert freeze-dried in a pre-cooled $(\mathrm{T}=77 \mathrm{~K}$ ) dessicator. For ultrastructural analysis in the electron microscope the sections were fixated using osmium tetraoxide $\left(\mathrm{OsO}_{4}\right)$ to enhance contrast. II quantitative results were to be obtained using EPMA, osmium fixation was omitted to avoid interference of the osmium $X$-ray lines with the elements of interest in the $X$-ray spectrum. In the latter case the cryosections were fixated with formalin yapour after freeze-drying in order to prevent rehydration of the fireeze-dried section. For details on the techniques used for the fixation of ultrathin sections the reader is referred to the work of Frederik et al. [FRE82]. For use in the electron microscope all sections were carbon coated in order to increase stability under electron bombardment.

\section{Histological analysis}

Light microscopy was performed on stained adjacent sections cut at a thickness of $10 \mu \mathrm{m}$. Since the spatial resolution of the light microscope in the proton microprobe set-up is limited due to the large working distance, a separate microscope was used in order to obtain pictures with better resolution (see Chapter 4 ).

\section{Proton probe analysis}

During all proton probe experiments the beam was operated in the dispersive mode in order to obtain the smallest possible beam dimensions. To identify the structures of the cryosections to be analyzed all sections were examined using STIM prior to quantitative analysis (PIXE and NBS / NFS). The regions of interest on the cryosection could be determined rapidly by comparison of the light microscope pictures from toluidine blue stained adjacent sections with the image obtained with the light microscope present in the microprobe set-up.

STIM analysis was performed using a $0.1 \mathrm{fA}$ proton bearn of about $\theta 1 \mu \mathrm{m}$ diameter. The beam was scanned across the specimen over an array of $128 \times 128$ pixels with a step size of $0.7 \mu \mathrm{m}$, thereby covering an area of about $90 \mu \mathrm{m} \times 90 \mu \mathrm{m}$. A measurement time of about 10 min was sufficient to obtain a two-dimensional image in which individual structures could be recognized. The beam dimensions during STIM analysis were checked routinely using a 2000 mesh Cu grid prior to analysis of the cryosections.

Following STIM analysis the object and aperture diaphragms were opened to obtain a beam of about $3 \mu \mathrm{m} \times 3 \mu \mathrm{m}$ with a beam current of 60-100 pA. During PIXE analysis exactly the same area was hit by the proton beam using a scan pattern of $32 \times 32$ pixels and by increasing the step size of the scan magnet from 0.7 to $2.8 \mu \mathrm{m}$ at the same time. The total deposited charge varied between 0.5 and $1.5 \mu \mathrm{C}$, corresponding with a total irradiation time of about $2-6 \mathrm{~h}$ per sample. To reduce beam induced sample damage during all experiments a $5 \mathrm{kHz}$ scan frequency was applied. The detector solid angles were determined using a $\mathrm{Ni}$ calibration foil as described in Chapter 4. The fitting program AXIL was used to fit the PIXE spectra, whereas the EGG program was used for the automatic analysis of NBS and NFS spectra (see Chapter 4). The 
elemental contents were calculated using the computer program PANEUT (see Chapter 4) in order to correct for thick target effects

As was mentioned in Section 4.2.2 the STMM technique can be used to localize individual cells within a freeze-dried tissue section. For this reason a special software routine has been written to calculate the mean elemental content of a selected area, such as a cell, within the total area scanned by the proton beam. This is accomplished by summing up the $\mathrm{X}$-ray spectra and NBS/NFS spectra of individual pixels within the region of interest. An additional benefit of this summation is the improvement of the counting statistics in the measured spectra. It therefore should be noted that this method is essentially different from calculating the mean cellular content after the content of each scanned pixel within the selected area has been calculated.

\section{Electron probe analysis}

Ultrathin sections were analyzed in a Philips CM-12 electron microscope using a $120 \mathrm{keV}$ electron beam with a beam current of about $0.3 \mathrm{nA}$. X-ray spectra were collected while the electron microscope was operated in the STEM-mode. During $2000 \mathrm{~s}$ an X-ray spectrum was obtained from an area of about $1 \mu \mathrm{m} \times 1 \mu \mathrm{m}$ irradiated by the electron beam before the sample was moved to another region of interest. Each time, besides spots on the tissue sections, the Formwar backing foil and a bare $\mathrm{Cu}$ grid were analyzed to provide the possibility to correct for extraneous contributions to the background in the X-ray spectrum (see also Chapter 3 ). The elemental contents were determined using calibration data obtained from PEG standards containing known amounts of $\mathrm{Na}, \mathrm{Mg}, \mathrm{P}, \mathrm{K}$ and $\mathrm{Ca}$. $\mathrm{X}$-ray spectra were analyzed using the $\mathrm{AXIL}$ software. Continuum counts were measured by integrating counts in the energy range of $4.50-$ $6.00 \mathrm{keV}$.

For histological purposes osmium tetraoxide stained sections were analyzed under the same conditions as during quantitative X-ray microanalysis. Although staining significantly improves contrast, in case unstained cryosections were used it was still possible to recognize the mitochondria within the tissue sections, thus allowing to measure the elemental content both inside the mitochondria as well as in the surrounding cytoplasm. In most cases the recognition of the mitochondria was facilitated due to the presence of black stains within these organelles. These black stains most likely represent the precipitation of calcium phosphate (see Section 6.2)

\section{Representation of data}

In the proton microprobe as well as in the electron microscope the elemental contents were all measured in dehydrated (freeze-dried tissue) sections. As a result in this chapter the calculated contents are expressed in $\mathrm{mg} \cdot \mathrm{kg}^{-1} \mathrm{dry}$ weight or ppm. This way the quantitative results from $\mathrm{PIXE}$ and EPMA analysis can be compared with each other. However, in order to compare the results from both proton and electron probe analysis with data from literature it is often preferred to express the contents in mmol/kg dry weight or as wet weight concentrations. Wet weight concentrations can be obtained by multiplying the dry weight concentrations by a conversion factor. In our work the value for this conversion factor is taken from the study of Sasaki and coworkers [SAS94] and amounts to (31 13.1$) \%$. This factor was determined from electron probe analysis of frozen hydrated sections taken from healthy rat hearts who were subsequently 
dehydrated inside the column of the electron microscope and again analyzed by the electron probe.

\subsection{Results}

Release of lactate dehydrogenase (LDH) duning the $\mathrm{Ca}^{2+}$-readmission period

The total $\mathrm{LDH}$ activity lost from the hearts varied from 198 till $275 \mathrm{U}$ per gram wet weight. Taking into account that the average rat heart contains $314 \mathrm{ULDH}$ per gram wet weight [VOR91] the conclusion can be drawn that on the order of 70-90\% of the cells have lost their $\mathrm{LDH}$ content during the $\mathrm{Ca}^{2+}$ paradox. Hence, the cell membranes of the majority of cardiomyocytes are severely damaged due to this manoeuvre.

\section{Histological analysis}

From light microscope analysis of stained sections it can be seen clearly that the tissue is severely damaged due to the calcium paradox intervention (see Fig. 6.3). In contrast with normoxic cardiac left ventricular tissue subjected to the calcium paradox shows no regular structure and the cells are not connected anymore and seem to be torn asunder. Moreover, the individual cells appear to have lost their rigid structure.
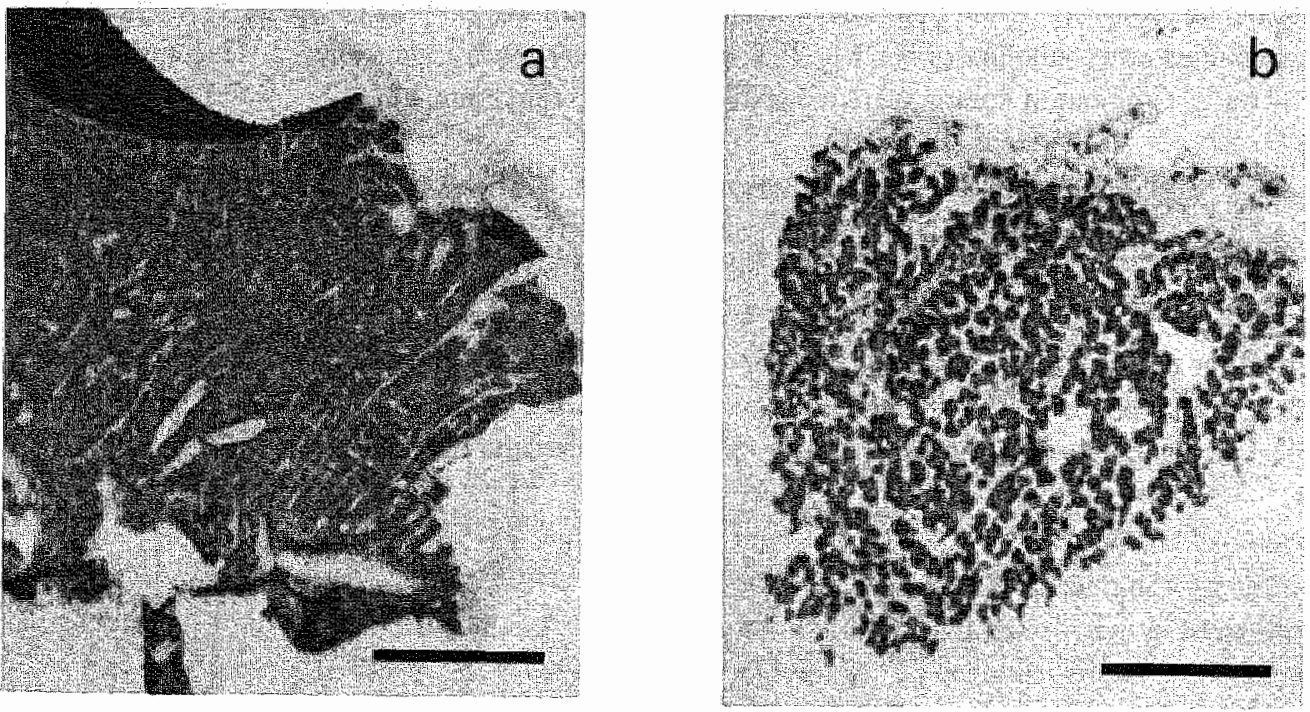

Figure 6.3: Ligh microscope pictwe of nomoxically perfised heart tissue (a) and tissue originating from a heart subjected to the calcium paradox manoewure (b). Note the almost complete lass of structure in the tissue after the calcium paradox mamoeuve. To improve contrast the cryosections were stained with toluidine blue. The tissue sections were cw at $10 \mu \mathrm{m}$ thickness. Bars represenv $0.5 \mathrm{~mm}$. 

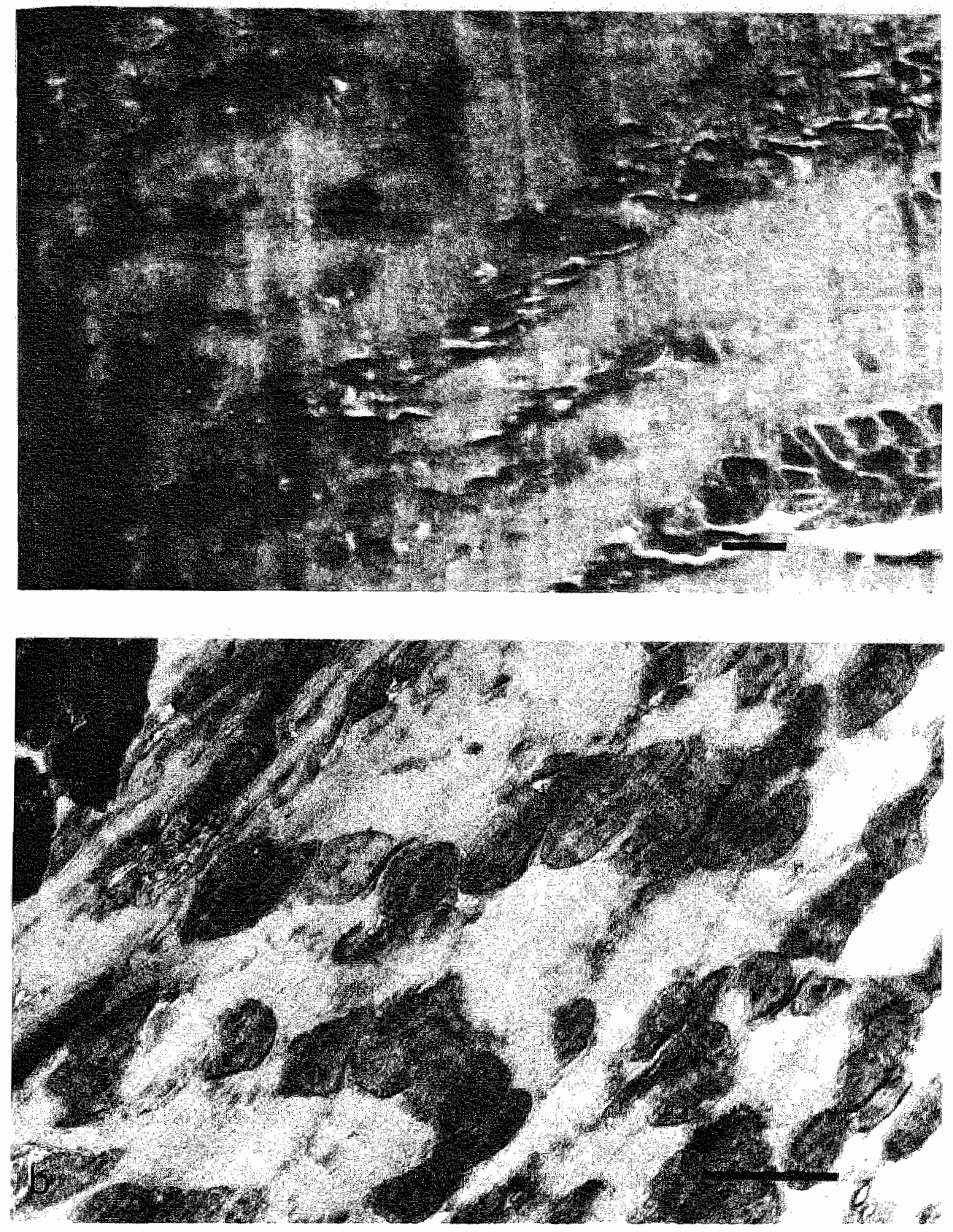

Figure 6.4: Electron microscope picture of normoxically perfused rat heart tissue (a) and tissue originating from a rat heart subjected to the calcium paradox manoeuvre (b). Nore the electron opaque deposits that are visible in the mitochondria in tissue subjected to the calcium paradox manoeuvre. To improve contrast the cryosections were fixed with osmium terraxide vapour. Utrathin sections were cut at 50.150 wm thickness. Bars represent $1 \mathrm{\mu m}$. 
Ultrathin ( $50150 \mathrm{~mm}$ ) sections of the same tissue were fixated and stained using $\mathrm{OsO}_{4}$ vapour to allow ultrastructural analysis. Electron microscope analysis of these sections shows that the mitochondra contain dense gramules (Fig. 6.4). These granules were not observed in tissue sections from control heants.

\section{Proton probe analysis results}

\section{a. Localization of myocardial cells using the STM rechique}

As was shown in Chapter 4, STIM can be used to localize individual cells within a freeze-dried cryosection. A typical result of the STMM analysis of thin tissue sections taken from a heart subjected to the calcium paradox is given in Figure 6.5 . Higure 6.5 a shows the result of a large scan covering an area of about $180 \mu \mathrm{m} \times 180 \mu \mathrm{m}$. From the STM picture it is again clear that the cells are not connected anymore and appear to be torn apart, resulting in large areas where no tissue is present. This result is in good agreement with the results of light microscope analysis of toluidine blue stained sections (Fig. 6.3).

a

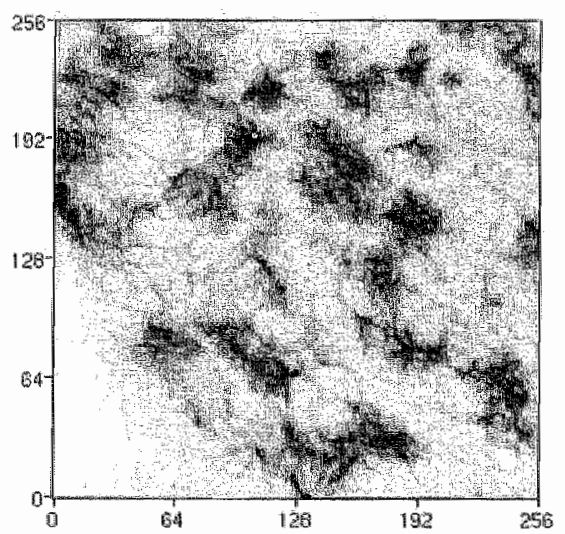

b

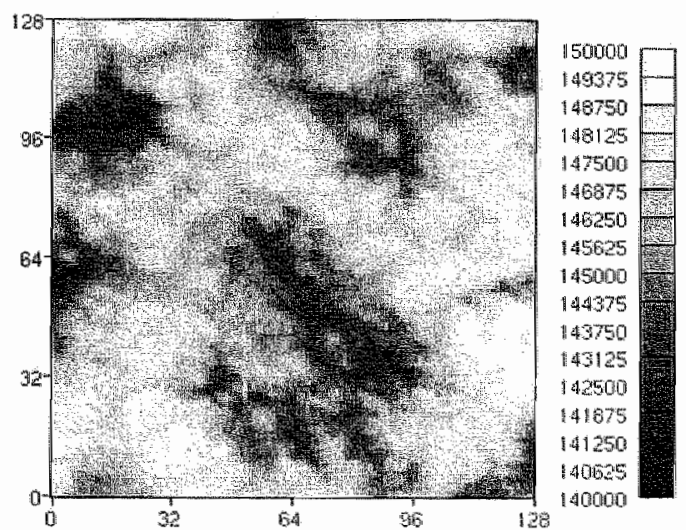

Figure 6.5: $\quad$ STM analysis of cardiac tissiue subjected to calcium paradox experimentix. The STIM scan covering a large area of about $180 \mu m \times 180 \mu m$ (a) shows the distupture of the tisstwe. A blow-1up of this area of $90 \mu m \times 90 \mu m(b)$ was obtained by reduction of the step size of the scan magnet and the mumber of scanmed positions.

After obtaining the STMM image, it is easy to select an interesting area for further PIXE and RBS/RFS analysis. However, as a result of the fragmentation of the tissue it was found to be very difficult to include as much as possible cellular material in a single (rectangular) scan during PIXE analysis. Therefore several measurements were performed using smaller scan areas. These preselected areas included as much as possible tissue in order to avoid extensive analysis of areas where no cellular material was present on the Formwar backing foil 

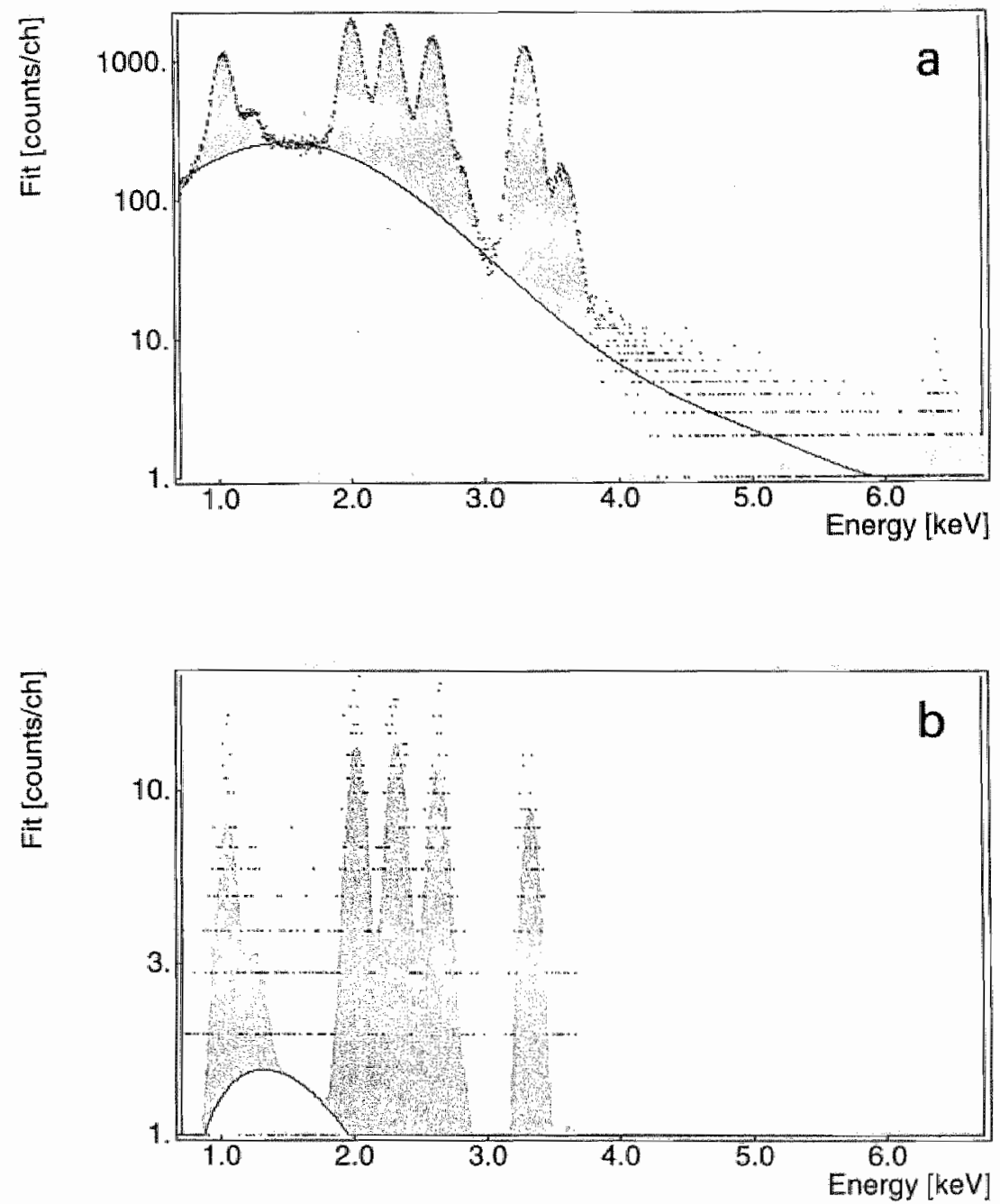

Figure 6.6: Typical example of an X-ray spectrum collected during irradiation of the summation of a whole cell, in this particular case consisting of 85 pixels (method 2)) (a), versus only one position (I pixel) in the scan pattern (b).

b. Quantitative proton probe analysis

From the data of proton probe analysis the elemental contents can be determined in two different ways. First the elemental content can be calculated for each position scanned by the proton beam (method 1). This way differences in content at the level of the dimensions of the proton beam are 
determined. Second, it is also possible to calculate the mean content of a region of interest (ROI) within the pattern scanned by the proton beam, such as an individual cell (method 2). It should be noted that in this case the energy spectra of the pixels present in the ROI are added up and subsequently this "sumspectrum" is used to calculate the mean elemental content. This is essentially different from calculating the mean content by taking the average of the content values for all pixels of the ROI, since by addition of the individual spectra better statistics are achieved (see Fig. 6.6).

A typical example of the calculated mass fraction distribution of several elements after the calcium paradox is shown in Figure 6.7. In the same figure the areal mass density distribution, determined from NBS / NFS analysis of the same area, is given in order to mark the spots where cardiac tissue is present. For most analyzed elements, such as $\mathrm{Na}, \mathrm{S}, \mathrm{K}$ and $\mathrm{Cl}$, the highest amounts of element are found at the places corresponding with the highest areal mass density. At these spots freeze-dried material, most likely corresponding with cell remnants, is present. It appears that the intracellular content of $\mathrm{Na}, \mathrm{Cl}, \mathrm{S}$ and $\mathrm{K}$ is distributed rather homogeneously within one single cell. Moreover, no appreciable differences in elemental content are found in case the cells or cell remnants that can be observed within the scanned area of about $90 \mu \mathrm{m} \times 90 \mu \mathrm{m}$ are compared. On the other hand, locally very high levels are found when the cellular content of $\mathrm{P}$ and $\mathrm{Ca}$ was analyzed. The highest $\mathrm{Ca}$ and $\mathrm{P}$ contents are observed at the outer borders of the cell remnants. In some cases it even appears that these "hot spots" lie in close vicinity, but not inside the cell remnants. Moreover, it seems that the positions where the highest $\mathrm{Ca}$ levels are found also contain large amounts of $\mathrm{P}$, suggesting precipitation of insoluble calcium phosphate crystals. These local elevations in $\mathrm{P}$ and $\mathrm{Ca}$ levels were not observed in tissue originating from normoxically perfused rat hearts (data not shown).

In Fig, 6.8 for $\mathrm{Na}, \mathrm{Mg}, \mathrm{P}, \mathrm{S}, \mathrm{Cl}, \mathrm{K}, \mathrm{Ca}$ and $\mathrm{Fe}$ the mean elemental content per cell is depicted for cells originating from both normoxically perfused hearts and hearts subjected to the calcium paradox. Comparing calcium paradox results. with those of normoxic rat heart results it is obvious that for all elements dramatic alterations in elemental contents are observed.

The $\mathrm{Na}$ content has increased from about $6,800 \mathrm{mg}^{-1} \mathrm{~kg}^{-1}$ in normoxic tissue up to 10,000 $17,000 \mathrm{mgkg}^{-3}$ after the calcium paradox. In addition to the increase in intracellular $\mathrm{Na}^{2}$ level, the cellular content of both $\mathrm{Cl}$ and $\mathrm{Ca}$ is much higher after the calcium paradox in comparison with values from normoxically perfused hearts. While the $\mathrm{Cl}$ levels increase from $10,000 \mathrm{mg}^{-k_{g}{ }^{-1}}$ up to about $30,000 \mathrm{mg}^{-1} \mathrm{~kg}^{-1}$, the mean cellular $\mathrm{Ca}$ content rises from about $500 \mathrm{mg} \cdot \mathrm{kg}^{-1}$ to values up to $2,000 \mathrm{mg}^{-1} \mathrm{~kg}^{-1}$.

The $\mathrm{Mg}$ content of the calcium paradox tissue, on the other hand, is very low in comparison with control values. In some cases the $\mathrm{Mg}$ content even has decreased to such an extent that the criterion for the limit of detection (see Chapter 2) was not satisfied. In some instances also $\mathrm{Fe}$ levels were below the minimum detectable concentration after the calcium paradox. In addition to $\mathrm{Mg}$ and $\mathrm{Fe}$ a decrease in elemental content is also observed for $\mathrm{P}, \mathrm{S}$ and $\mathrm{K}$. From these elements the most dramatic decline is found for the $\mathrm{K}$ content, which decreases from about $15,000 \mathrm{mg}^{\mathrm{kg}}{ }^{-1}$ for normoxically perfused hearts down to values between $2,000-4,000 \mathrm{mg} \cdot \mathrm{kg}^{-1}$ after the calcium paradox 


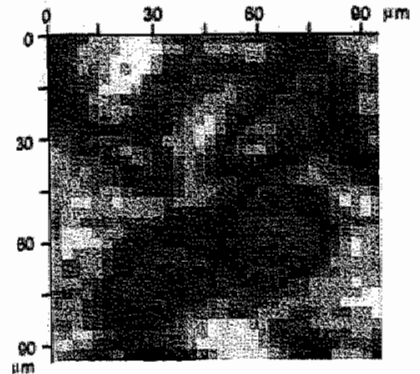

$\rho_{s} t$

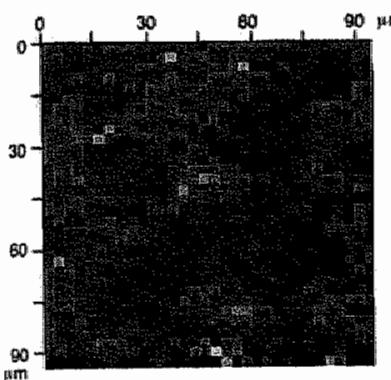

P

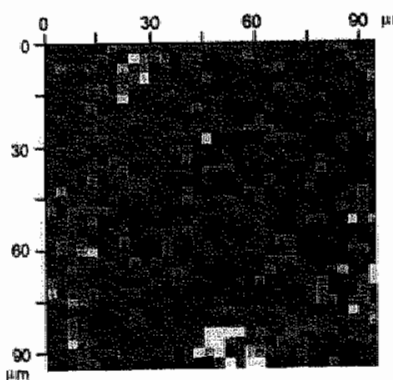

K.

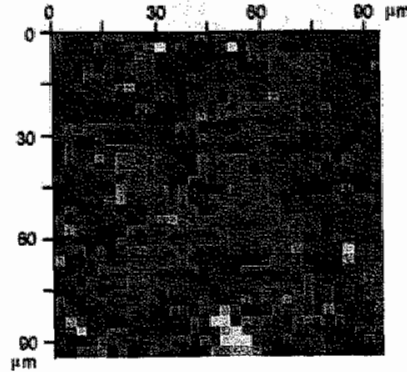

$\mathrm{Na}$

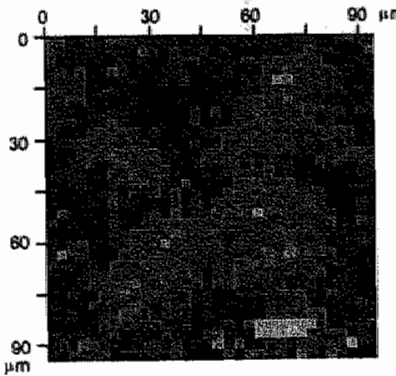

S

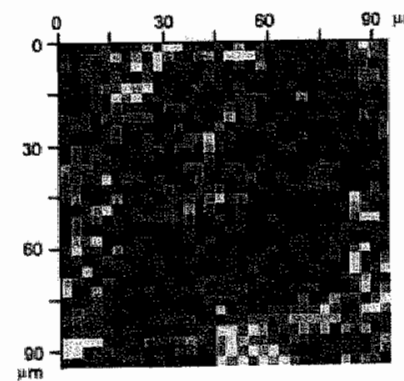

$\mathrm{Ca}$

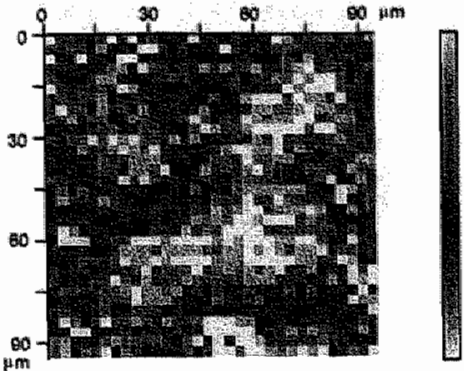

$\mathrm{Mg}$

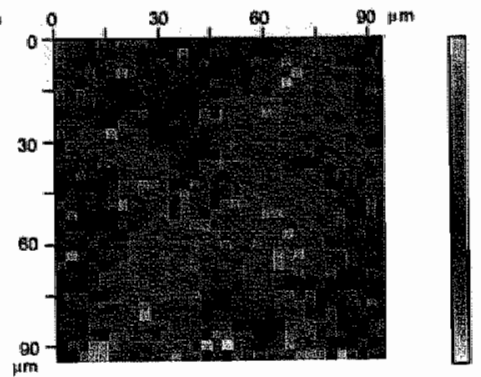

$\mathrm{Cl}$

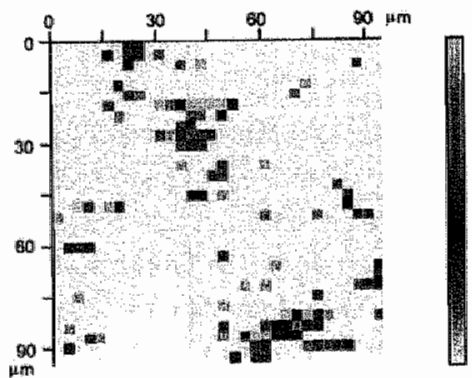

$\mathrm{Fe}$

Figure 6.7: Distributions of the $\mathrm{Na}, \mathrm{Mg}, \mathrm{P}, \mathrm{S}, \mathrm{Cl}, \mathrm{K}, \mathrm{Ca}$ and Fe content (calculated wsing method I (see text)) in cardiac tisste subjected to the calcium paradox. The areal mass density $\left(\rho_{s} t\right)$ distribution (upper left corner) was obrained from NBS / NFS analysis and corresponds with the presence of freeze-dried tissue. The size of one pixel corresponds with about $3 \mu \mathrm{m} \times 3 \mu \mathrm{m}$. 

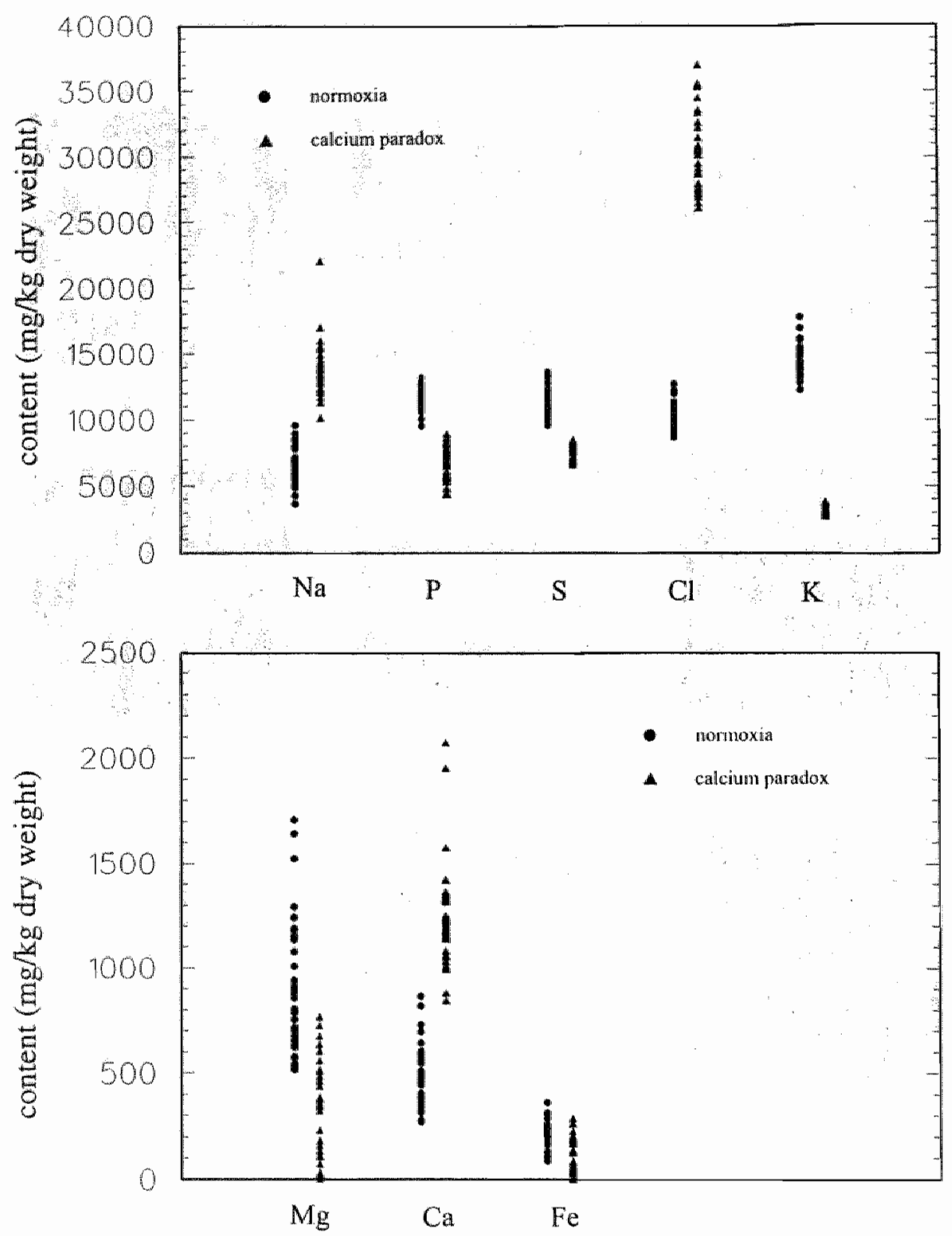

Figure 6.8: Mean elemertid content (calculated using method 2 (see text)) of individual cells in both control tissue and calcium paradox tissue. Each point in the figure represents the mean coment of a region of interest within the total scan pattern, corresponding with one single cell or cell remmam. Note the differences in the two scales. Contents are expressed in $m g \mathrm{~kg}^{-1}$ dry werght. 
Next to the fact that for all elements large differences in average cellular content between normoxic and calcium paradox treated cells are found, it also apparent from Fig. 6.8 that for most elements the scatter in both control values and calcium paradox values is not so large. This may also be obvious from the coefficient of variation CV, defined as (S.D./ X) (see Table 6.1).

Table 6.I Mean values \pm S.D. of the Na, Mg, P, S, Cl, K, Ca and Fe content of individual cells or cell remnants in control tisswe $(n=35)$ and in cellular material after calcium paradox $(n=32)$. In addition the coefficient of variation, defined as $(S . D / X)$, is shown. Three rat hearts were normoxically perfitsed and five hearts were stbjected to the calcium paradox manoewre. From each heart about $1-2$ sections were analyzed. Preselected areas of $90 \mu \mathrm{m} \times 90 \mathrm{\mu m}$ were analyzed by the scanning proton beam and generally contained about $\$-5$ cells or cell remnants. The cells were analyzed irrespective of heart or tissue section.

\begin{tabular}{|c|c|c|c|c|c|c|c|c|}
\hline & $\mathrm{Na}$ & $\mathrm{Mg}$ & $p$ & $s$ & $\mathrm{Cl}$ & $k$ & $\mathrm{Ca}$ & Fete \\
\hline calcium paradiox & $83,720=2,110$ & $335 \pm 227$ & $6,695: 1,255$ & $7,645 \pm 620$ & $30,980 \div 4,210$ & $3,070 \div 31.5$ & $1.245 \pm 235$ & $95 \pm 85$ \\
\hline S.D. $/ X$ & 0.15 & 0.68 & 0.19 & 0.08 & 0.14 & 0.10 & 0.21 & 0.93 \\
\hline marnoxia & $6,755=1,315$ & $915 \pm 320$ & $11,550 \pm 680$ & $11,200 \pm 1,065$ & $10,440 \pm 950$ & $14,4+5=1,240$ & $490=145$ & $10 \div 60$ \\
\hline S.D. IX & 0.19 & 0.35 & 0.06 & 0.10 & 0.09 & 0.09 & 0.30 & 0.29 \\
\hline
\end{tabular}

After the calcium paradox for most elements a rather low coefficient of variation is found $(<0.20)$, except for $\mathrm{Mg}(\mathrm{CV}=0.68)$ and $\mathrm{Fe}(\mathrm{CV}=0.93)$. These high values most likely do not reflect a substantial heterogeneity in cellular $\mathrm{Mg}$ and $\mathrm{Fe}$, but are simply due to the very low amount of these elements present in the tissue sections analyzed, resulting in a high statistical uncertainty. Besides, for most elements no overlap in content between the two conditions is observed, i.e., after calcium paradox the elemental content of all cells or cell remnants $(n=32)$ has changed with respect to normoxic tissue. The small coefficient of variation of $\mathrm{Na}, \mathrm{P}, \mathrm{S}, \mathrm{Cl}$, $\mathrm{K}$ and $\mathrm{Ca}$ strongly indicates that all analyzed cells in hearts subjected to the calcium paradox are damaged to more or less the same extent when the elemental content is used as a measure of cellular damage.

\section{Electron microprobe resulls}

In contrast with the proton probe the superior spatial resolution of the electron microscope offers the possibility to recognize individual subcellular organelles within ultrathin sections of cardiac tissue. In order to detect intracellular heterogeneities in elemental contents both mitochondria and their surrounding environment were analyzed, thus allowing the comparison of the content of 
several elements insicle the mitochondria with those of the surrounding tissue. This is of special interest, since it is well known that mitochondria act as a store for calcium in case cytoplasmic $\mathrm{Ca}^{2+}$ levels become too high [P[E95].

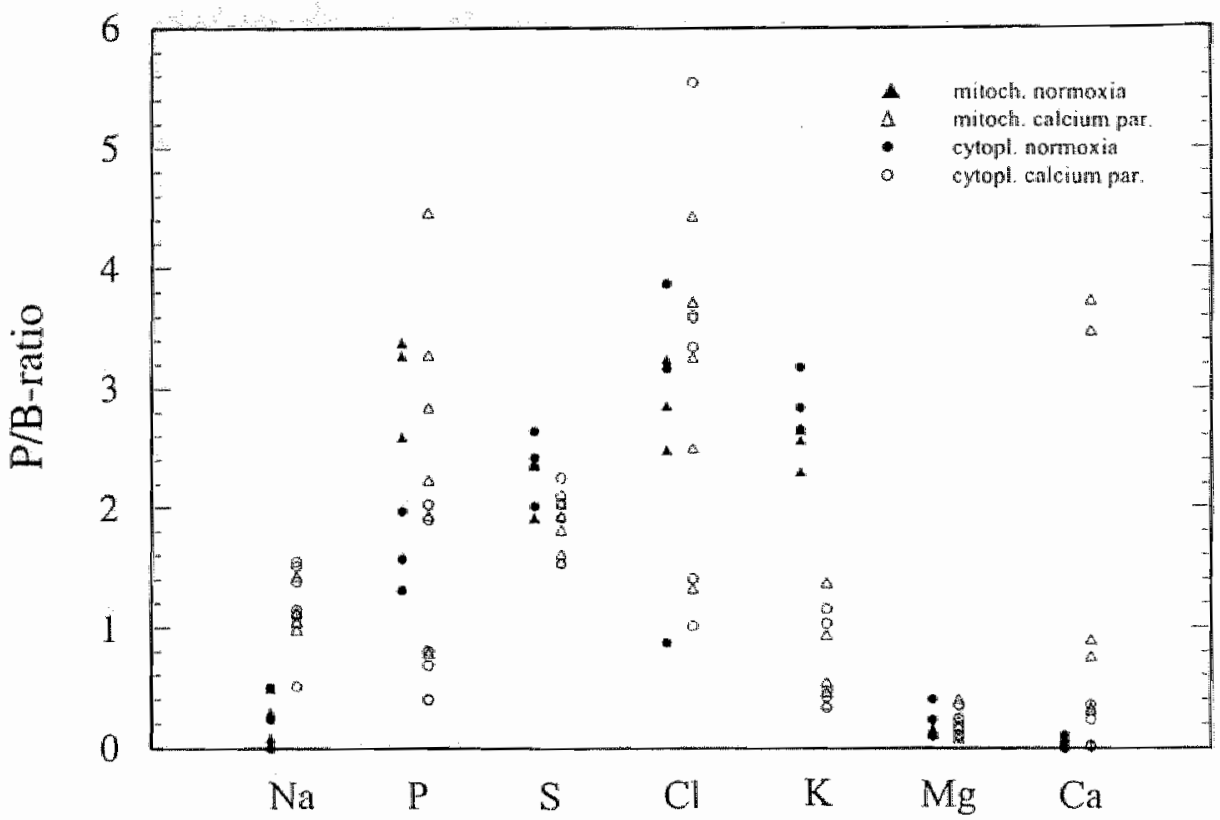

Figure 69: Results from EPMA analysis of mitochondria and cytoplasm in normoxic hearts and hearis swbjected to the calcium paradox. For each condition studied only a few sections, originating from $1-2$ hearts, were analyzed. Each poim in the figure corresponds with an area of about $1 \mathrm{\mu m} x$ I $\mu \mathrm{m}$ scanned by the electron beam. In control tissue 3 mitochondria and 3 spots in cyloplasm were analyzed whereas 6 mitochondria and 6 spors in cyroplasm were analyzed in calcium paradox induced rissue, respectively.

The results of the EPMA analysis of damaged tissue and control tissue are documented in Fig. 6.9. For each element both control and calcium paradox values are given. Moreover, within each condition a distinction has been made between mitochondria and cytoplasm. Although the number of analyzed spots is limited, some tendencies became manifest. In the first place it is notable that for $\mathrm{Na}, \mathrm{Mg}$ and $\mathrm{K}$ in both normoxic and calcium paradox tissue no differences in content are found between the cytoplasm and the mitochondria. On the contrary, in both conditions $\mathrm{Ca}$ and $\mathrm{P}$ levels in the mitochondria are significantly higher than in the surrounding cytoplasm.

By comparison of the two conditions it is evident that the $\mathrm{Na}$ content has increased from roughly $1,800 \mathrm{mg} \cdot \mathrm{kg}^{\prime}$ in control tissue to about $6,500 \mathrm{mg} \cdot \mathrm{kg}^{-1}$ after the calcium paradox. An even more dramatic increase is observed for $\mathrm{Ca}$ with a mean mitochondrial content of about 
$6,000 \mathrm{mg} \cdot \mathrm{kg}^{-1}$. In addition in some cases about $1,200 \mathrm{mg} \cdot \mathrm{kg}^{-1} \mathrm{Ca}$ is found in the cytoplasm after calcium paradox, whereas for control tissue the calcium content was below the limit of detection $\left(\sim 800 \mathrm{mg} \cdot \mathrm{kg}^{-1}\right)$ for both cytoplasm and mitochondria. In contrast with $\mathrm{Na}$ and $\mathrm{Ca}$ the $\mathrm{P}$ and $\mathrm{K}$ levels in both cytoplasm and mitochondria have decreased after the calcium paradox. The $\mathbb{K}$ content has decreased from about $10,000 \mathrm{mg} \mathrm{kg}^{-1}$ down to $2,000-3,000 \mathrm{mg} \cdot \mathrm{kg}^{-1}$. Although a decline in the mean $P$ content is observed, some mitochondria still contain very high $P$ levels after the calcium paradox. In contrast with the results from proton probe analysis, no decline in $\mathrm{Mg}^{2+}$ could be observed in the calcium paradox treated hearts. This unexpected finding is probably due to the high statistical uncertainty in the determination of the Mg peak content in the X-ray spectrum obtained during EPMA analysis, because of the low Mg content of the tissue section.

At the time the EPMA measurements were performed no standards for $\mathrm{S}$ and $\mathrm{Cl}$ were available. Nevertheless the peak-to-background $(\mathrm{P} / \mathrm{B})$ ratio can be used in order to get a qualitative impression of the alterations of these elements as a result of the calcium paradox. For $\mathrm{S}$ a minor decrease in $\mathrm{P} / \mathrm{B}$-ratio was observed. On the contrary, for $\mathrm{Cl}$ the $\mathrm{P} / \mathrm{B}$-ratio slightly increased from a mean value of 2.74 for control tissue up to 3.12 after the calcium paradox.

\subsection{Discussion}

In this study several analytical techniques to monitor the induced damage in hearts subjected to calcium paradox have been applied. From the assessment of the $\mathrm{LDH}$ release during the $\mathrm{Ca}^{2+}$ readmission period it could be concluded that the cell membrane of at least the majority of cardiomyocytes is severely damaged due to the calcium paradox manoeuvre. For this reason it is expected that appreciable changes in elemental content can be observed in case tissue sections from hearts subjected to the calcium paradox are compared with tissue sections from normoxically perfused hearts.

Before the results from proton and electron probe X-ray microanalysis are discussed it should be realized that large differences exist between these techniques, such as sample size, thickness of the cryosections, process of freeze-drying, dimensions of the area hit by the particle beam, calibration method used, etc. All these factors may have a substantial impact on the absolute values of the elemental contents found in the analyzed tissue. Despite these differences in general comparable tendencies were apparent.

\section{Discussion of proton probe results}

- It seems probable that the dramatic increase in the cellular sodium content can be explained by the fact that this element can freely enter the cell after the cellular membrane has been damaged, since the sarcolemma of an injured cell is no longer a barrier for intra- and extracellular ions and other components. It therefore is expected that at the end of the $\mathrm{Ca}^{2 *}$ readmission period the intracellular $\mathrm{Na}$ content has levelled with the extracellular $\mathrm{Na}$ concentration. The mean cellular sodium content of $13,000 \mathrm{mg} \cdot \mathrm{kg}^{-1} \mathrm{dry}$ weight corresponds to $170 \mathrm{mmol} / \mathrm{l}$ in case a conversion factor of 0.31 is used. This value is in good agreement with the value for the $\mathrm{Na}$ concentration of the crystalloid buffer solution, which amounts to $160 \mathrm{mmol} / \mathrm{l}$. 
The gain in intracelluar $\mathrm{Na}^{*}$ due to the calcium paradox manoeuvre was already established earlier by Alto and Dhalla [ALT79] and Nayler and Grinwald [NAY81]. In a later study Nayler and colleagues [NAY84] found that after $10 \mathrm{~min}$ of $\mathrm{Ca}^{2+}$ depletion, followed by 10 min of $\mathrm{Ca}^{2+}$ repletion, rat hearts contained about $210 \mu \mathrm{mol} / \mathrm{g} \mathrm{dry}$ weight of $\mathrm{Na}^{*}$. This value, as assessed with flame photometry, corresponds with about $5,000 \mathrm{mg} \cdot \mathrm{kg}^{-1} \mathrm{dry}$ weight. In comparison with $\mathrm{Na}^{+}$control values of about $1,300 \mathrm{mg}^{\circ} \mathrm{kg}^{-1} \mathrm{dry}$ weight this is almost a 4 -fold increase. It thus appears that, although a discrepancy exists between the absolute sodium values found in the study of Nayler er al. and the work presented in this chapter, the extent of the rise in the sodium content agrees reasonably well.

- One of the most striking findings was the very low magnesium content of the intracellular space after the calcium paradox. In several samples it was even impossible to detect magnesium, i.e., the magnesium content was below the limit of detection of the proton microprobe, even if the sum of the PIXE spectra corresponding with all pixels within a selected area was analyzed instead of the spectrum of one single position scanned by the proton beam For an accumulated charge of about $1 \mathrm{nC}$ and a sample thickness of about $0.3 \mathrm{mg} / \mathrm{cm}^{2}$ this means that the magnesium content of the cardiac tissue after calcium paradox was lower than $80 \mathrm{mg} / \mathrm{kg}$ dry weight, corresponding with about $3 \mathrm{mmol} / \mathrm{kg}$ dry weight. Kirkels and coworkers [KIR89], who measured the release of magnesium from the heart using atomic absorption spectrophotometry (AAS), reported a cumulative $64 \%$ loss of total rat heart $\mathrm{Mg}^{2+}$ after 5 min of $\mathrm{Ca}^{2+}$-free perfusion followed by $5 \mathrm{~min}$ of normal perfusion. They found that after calcium paradox only about $14 \mathrm{mmol} / \mathrm{kg}$ dry weight of $\mathrm{Mg}^{2+}$ was left in the rat heart. The fact that in our study both the $\mathrm{Ca}^{2+}$-free perfusion period as well as the $\mathrm{Ca}^{2+}$-readmission period were longer as compared with the study of Kirkels et al. [KIR89] might be responsible for the even more dramatic loss of magnesium from the intracellular compartment.

- After calcium paradox the P content has decreased from about $11,500 \mathrm{mg} \cdot \mathrm{kg}^{-1}$ dry weight in control tissue to levels between 4,000 and $9,000 \mathrm{mg}^{-1}$. Since $P$ is present in both the sarcolemma, which contains appreciable amounts of phospholipids (PL), and in cytoplasmic inorganic phospluates $\left(\mathrm{P}_{i}\right)$ and energy-rich phosphates, such as ATP, guanosine triphosphate (GTP) and phosphocreatine (PC), this decline might be attributed to the loss of these elements. De Groot and coworkers [GRO92], who studied the nucleotide changes in pre-ischemic, ischemic and reperfused rat hearts, found that the pre-ischemic rat heart contains about 20, 17 and $1 \mu \mathrm{mol} / \mathrm{g}$ dry weight PC, ATP and GTP, respectively. It should be noted that $1 \mathrm{~mol}$ of both ATP and GTP contains $3 \mathrm{P}$ atoms. According to Van Bilsen er al. [BIL88] the total PL content of the rat heart amounts to $178 \mu \mathrm{mol} / \mathrm{g}$ dry weight, whereas the total pre-ischemic $P_{i}$ amounts to about $34 \mu \mathrm{mol} / \mathrm{g}$ dry weight. It thus follows that the total $\mathrm{P}$ content of the rat heart amounts to $286 \mu \mathrm{mol} / \mathrm{g}$ dry weight $(20+51+3+178+34)$, corresponding with about $9,000 \mathrm{mg} \cdot \mathrm{kg}^{-1}$ $\mathrm{dry}$ weight. This calculated value is in the same order of magnitude as the $\mathrm{P}$ content measured in the present study. Van der Vusse and colleagues [VUS88], who studied the myocardial content of fatty acids and phospholipids during the calcium paradox, did not observe a substantial decline in the tissue content of phospholipids after repletion of $\mathrm{Ca}^{2+}$. As a result the conclusion can be drawn that the decline in $\mathrm{P}$ levels most likely cannot be attributed to a biochemical degradation of the myocardial membrane-lipids. From literature [KAT92] it is 
known, however, that the hydrolysis of ATP and phosphocreatine liberates large amounts of phosphate which rapidly leaves the cell. This implies that in our case, making use of the values for the $\mathrm{P}$ content as presented above, about $38 \%$ of the total myocardial $\mathrm{P}$ content is lost. This value agrees reasonably well with the result from the proton microprobe analysis of the mean $P$ content before and after the calcium paradox manoeuvre

- A strong decline in the cellular $S$ content is observed after the calcium paradox. Since $S$ is bound to proteins (disulfide bounds) this element can only leave the intracellular compartment if these macromolecules are able to pass the injured cell membrane. Since a massive loss of LDH from the cells was observed, indicating that the cellular membranes have been disrupted, it is very likely that the efflux of proteins and thus of $S$ is caused by the injury inflicted upon the sarcolemma.

- In comparison with normoxic tissue the intracellular $\mathrm{Cl}$ content has increased considerably to values of about $30,000 \mathrm{mg} \cdot \mathrm{kg}^{-1} \mathrm{dry}$ weight, corresponding with $254 \mathrm{mmol} / 1$. In contrast with the sodium concentration this value exceeds the $\mathrm{Cl}$ concentration $(142.4 \mathrm{mM})$ of the buffer solution. It thus appears that once the sarcolemma no longer is able to act as a barrier between the intra- and extracellular compartments $\mathrm{Cl}$ accumulates in the cell remnants. The fact that the resulting $\mathrm{Cl}$ content is higher than expected from the $\mathrm{Cl}$ concentration of the perfusion medium might be attributed to the binding of this anion to positively charged proteins that are still present in the intracellular compartment, such as the contractile proteins and/or proteins of the intracellular matrix.

- The dramatic decrease in the cellular $\mathbb{K}$ content can be attributed to the efflux of this element from the intracellular compartment after loss of membrane impermeability. This $\mathrm{K}$ loss was assessed earlier by Alto and Dhalla [ALT79], who studied the myocardial cation content during induction of calcium paradox using atomic absorption spectrophotometry. They found that after $10 \mathrm{~min}$ of $\mathrm{Ca}^{2+}$ free perfusion, followed by reperfusion with a calcium containing buffer, the $\mathrm{K}^{+}$content had decreased to $180 \mu \mathrm{mol} / \mathrm{g}$ dry weight, corresponding with about 7,000 $\mathrm{mg} \cdot \mathrm{kg}^{-1} \mathrm{dry}$ weight. This value still exceeds the $2,000-4,000 \mathrm{mg} \cdot \mathrm{kg}^{-1} \mathrm{~K}$ found in our study. However, it should be noted that in the study presented in this chapter the $\mathrm{Ca}^{24}$ repletion period lasted $10 \mathrm{~min}$, whereas in the study of Alto and Dhalla [ALT79] this period lasted only $5 \mathrm{~min}$. - In contrast with potassium calcium levels have increased significantly after calcium paradox. In this study it was observed that the mean cellular $\mathrm{Ca}$ content increased to about 1,000-2,000 $\mathrm{mg} \cdot \mathrm{kg}^{-1} \mathrm{dry}$ weight after $\mathrm{Ca}^{24}$ repletion. This values agrees reasonably well with the value found by Nayler and coworkers [NAY84] of about $27 \mu \mathrm{mol} / \mathrm{g}$ dry weight, corresponding with $\sim 1,100 \mathrm{mg} \cdot \mathrm{kg}^{-1} \mathrm{dry}$ weight. This value was assessed by means of atomic absorption spectrophotometry. It appeared that for Ca locally very high levels were found that were significantly higher than the mean cellular $\mathrm{Ca}$ content. It is believed that these locally elevaled Ca levels can be attributed to the massive accumulation of calcium ions in the mitochondrial compartment of the injured cell, since the mitochondria serve as $\mathrm{Ca}^{2+}$ scavengers in situations. of elevated cytoplasmic calcium levels [KAT92]. This was confirmed by EPMA analysis of mitochondria in tissue sections from hearts subjected to the calcium paradox (see next page)

- Along with $\mathrm{Mg}$ the Fe content had decreased to such an extent that in some cases the limit of detection criterion was not met for these elements. The decline in Fe content can be explained 
by the loss of myoglobin from the heart during $\mathrm{Ca}^{2 *}$-readmission. This explanation is supported by the wisual observation that the heart turns pale during $\mathrm{Ca}^{2+}$ readmission

In the distributions of the elemental content next to the cell remnants also the extracellular environment is visible. It was shown in both Figs. 6.3 and 6.4 that as a result of the calcium paradox manoeuvre the tissue is damaged to such an extent that the cells are not connected anymore. As a result large areas of extracellular space are present between the cell remnants. If the extracellular compartment is filled with buffer solution at the time of cryofixation, the elements present in the extracellular space are expected to precipitate on the supporting Formvar foil after subsequent freeze-drying. Since the amount of element present is normalized on the total areal mass density very high "extracellular" contents are to be expected due to the low mass thickness of the Formvar foil. However, from Fig. 6.7 it follows that for all elements the extracellular content is very low and is much lower than the elemental content in the tissue sections. For this reason it is assumed that an appreciable amount of extracellular fluid is lost from the biopsy between excision from the heart and subsequent cryofixation or (less likely) that the elements present in the extracellular space (precipitated as salts) simply are lost after freeze-drying during sample handling procedures.

\section{Discussion of electron probe results and comparison with proton probe results}

Just like proton probe analysis EPMA showed an increase in $\mathrm{Na}, \mathrm{Cl}$ and $\mathrm{Ca}$ content and a decrease in $\mathrm{P}$ and $\mathrm{K}$ levels after the calcium paradox. However, for EPMA lower absolute values for $\mathrm{Na}$ and $\mathrm{P}$ were found in both control tissue and calcium paradox tissue, whereas the $\mathrm{K}$ contents for both stages agree reasonably well in case the two techniques are compared. A passible explanation for the discrepancy has not been found. In this respect it is notable that both $\mathrm{Na}$ and $\mathrm{P}$ contents are calculated making use of the results of EPMA analysis of a standard containing both $\mathrm{Na}$ and $\mathrm{P}$. In contrast with the $\mathrm{K}$ and $\mathrm{Ca}$ standards, the $\mathrm{Na}$ and $\mathrm{P}$ standard was not analyzed in the proton microprobe in order to check for preparation artefacts.

It is also striking that a decrease in the $\mathrm{Mg}$ content was only observed with the proton probe. Moreover, in contrast with the proton probe both $\mathrm{Ca}$ and $\mathrm{Fe}$ could not be detected in normoxic tissue using the electron probe. With EPMA the presence of calcium could only be observed after calcium paradox, since in this case obviouslly highly elevated levels occur.

It appeared that the absolute value of the local calcium content inside the mitochondria measured with the electron probe is significantly higher than the highest values found with the proton probe. This can be explained as follows. As a result of the relatively large spot size of the proton probe $(3 \mu \mathrm{m} \times 3 \mu \mathrm{m})$ combined with a sample thickness of about $3 \mu \mathrm{m}$ after freeze-drying, the total sample volume irradiated by the proton a beam at a certain position of the scan pattern contains both mitochondria and cytoplasm. This means that it is almost impossible to analyze solely an isolated mitochondrion with the current probe size of the Eindhoven proton microprobe. The resulting calcium content determined at a spot where mitochondria are present is therefore always an average value of the calcium content in the mitochondria and the content of the surrounding environment. 


\subsection{Summary and concluding remarks}

From the measurements described above it can be concluded that the calcium paradox manoeuvre is a useful experimental model to create an appreciable imbalance in the ion homeostasis of the cells. Both proton and electron probe analysis showed dramatic alterations in the cellular elemental content in tissue preparations from hearts subjected to the calcium paradox. For most elements a small coefficient of variation, i.e., $<20 \%$ was found, indicating that the insult in the calcium paradox experiments created a comparable alteration in elemental content in all analyzed cells. Considering the fact that 32 individual cells originating from 5 rat hearts were analyzed, this suggests that with respect to alterations in elemental content all hearts were damaged to approximately the same extent during calcium paradox. The observed changes in elemental content most likely serve as an indicator of the maximum alterations that are to be expected after ischemia and subsequent reperfusion (see next chapter). It also appeared that the proton probe should be used if the mean elemental content of different cells has to be determined with good sensitivity, whereas the electron microprobe is preferred in case subcellular resolution is required. 
Chapter 6 


\section{Chapter 7}

\section{Cellular and intracellular heterogeneity of elemental content in normoxic, ischemic and reperfused rat hearts}

\subsection{Introduction}

The function of ionic pumps present in cellular mermbranes is impaired in case the supply of substrates and oxygen is stopped. In the heart this condition is created by a reduction of blood flow through the coronary vessels (e.g. ischemia). For this reason ischemia will result in an appreciable imbalance in the ion concentrations of the oxygen-deprived nyocardial cells. The cardiomyocytes will inevitable die unless flow is restored in due time. Although additional damage might be inflicted upon the heart during reperfusion (reperfusion injury), timely restoration of supply conditions allows structural and functional recovery of the myocardial cells. However, when the ischemic period is prolonged a phase will be reached after which ischemic injury becomes irreversible. Under experimental conditions cell death begins after 15 to $40 \mathrm{~min}$ of total ischemia. It thus follows that in the time course of injury development due to ischemia/reperfusion different stages can be distinguished (see Fig. 7,1).

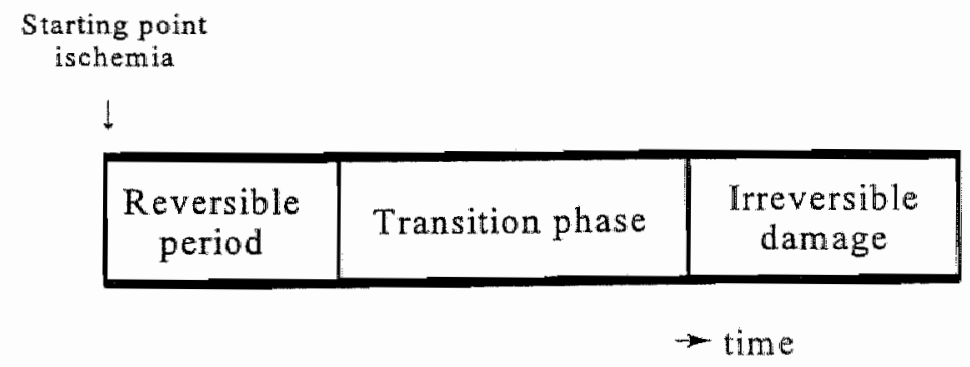

Figure 7.1 Schemoric representation of the course of injury development dhe to ischemia and reperfusion.

After a period during which all cells will recover from the ischemic insult upon restoration of flow (reversible phase), a transition period can be distinguished. During this period, part of the myocardial cells will die, whereas other cells are still able to recover when supply conditions are restored. Finally, when the ischemic period is prollonged a "point of no return" will be passed and all cells will be damaged irreversibly [PIP90].

Since the disrupture of the sarcolemma is generally suggested as being the proximate cause of ischemic cell death, the following definition of irreversible injury can be applied, ile.e, the moment that the myocardial cell membrane is no longer able to act as a barrier between the intracellular compartment and the extracellular space. If we adopt this delinition, release of macromolecules or enzymes from the heart can be used as an indicator of irreversible injury. However, this criterium is found to be positive only when cells have been damaged irreversibly 
and gives no information about the alterations that precede the "point of no-return". Several authors [REI86, BUJ86] reported that inhibition of the NaK pump in the ischemic myocardial cell results in a lower $\mathrm{Na}^{*}$ gradient across the sarcolemma, which in turn causes the influx of $\mathrm{Ca}^{2+}$ into the intracellular space through activation of the $\mathrm{Na}^{+}-\mathrm{Ca}^{2+}$ exchanger. Impairment of the Na-K ATPase activity will also result in a net loss of potassium from the myocardial cells. Since an imbalance in both intra- and extracellular content of elements, such as $\mathrm{Na}, \mathrm{Mg}, \mathrm{K}$ and $\mathrm{Ca}_{\text {, }}$ is already created in an early stage of ischemia, the alterations in the content of these elements are most likely more informative about the process that eventually leads to irreversible myocardial injury than the release of macromolecules or enzymes from the heart.

In summary, from the above it follows that the changes in intracellular content of these elements might play an important role in the onset of events that lead to irreversible damage. Therefore it can be concluded that there is a dual significance of these elements; alterations in intracellular elemental content (i) can be used as an indicator of the injury inflicted on the myocardial cell and (ii) might play a role in the induction of irreversible injury.

\section{Aim of the situdy}

In Chapter 5 preliminary results of proton microprobe analysis of control, normoxic, ischemic and reperfused rat hearts have been presented. The experiments described in Chapter 5 showed that alterations in $\mathrm{Na}, \mathrm{Mg}, \mathrm{K}$ and $\mathrm{Ca}$ concentrations could be followed with a spatial resolution in the order of a few $\mu \mathrm{m}$. From these experiments it was also inferred that two major improvements should be introduced in order to obtain more reliable results at the cellular level. First, the introduction of the STIM technique should facilitate to localize individual cells within the freeze-dried cryosections. Secondly, the freeze-dried tissue sections should be treated with more caution to reduce the possible detrimental effects of rehydration of the sections on the distribution of the elements of interest.

In the previous chapter the alterations in mean cellular content in heart tissue during the calcium paradox manoeuvre, a condition leading to severe damage of the myocardial cells, were discussed. Accordingly, the changes in elemental content in hearts subjected to this manoeuvre are indicative of the maximum changes that are to be expected when the heart is injured irreversibly. In the present chapter the results from the proton and electron microprobe analysis of control, normoxic, ischemic and reperfused rat heart tissue will be presented.

The objectives of this study are:

- to determine the mean $\mathrm{Na}, \mathrm{Mg}, \mathrm{P}, \mathrm{S}, \mathrm{Cl}, \mathrm{K}, \mathrm{Ca}$ and $\mathrm{Fe}$ content of myocardial cells in control, normoxic, ischemic and reperfused rat heart tissue by means of the combined use of PIXE and STIM and to compare the cellular elemental content of the four different stages.

- to compare the mean elemental content of individual cells within each analyzed stage in order to study whether (i) cellular homogeneity is found in both control and normoxically perfused rat hearts and (ii) the imposed cellular damage is the same for all affected cells or cellular heterogeneity is found after ischemia and reperfusion.

- to determine whether local, intracellular variations in elemental concentration can be observed. To this end both the proton and the electron microprobe were used. 
- to compare the results from this study with the results from calcium paradox experments (see Chapter 6), since alterations in the cellular content are expected to be less dramatic upon short-term ischemia/reperfusion than during the calcium paradox.

\subsection{Materials and methods}

\section{Perfusion protocol and sample preparation}

Animal experiments were performed on 13-14 weeks old male Lewis rats. The animals were anaesthetized with Hypnorm $(0.01 \mathrm{mg} / 100 \mathrm{~g}$ body weight fentanyl plus $0.5 \mathrm{mg} / 100 \mathrm{~g}$ body weight fluanisone s.c.) and the hearts were rapidly removed from the thorax after cervical dislocation. Subsequently, the hearts were connected via the aorta to the perfusion apparatus as described in Chapter 5 .

Tissue biopsy sampling

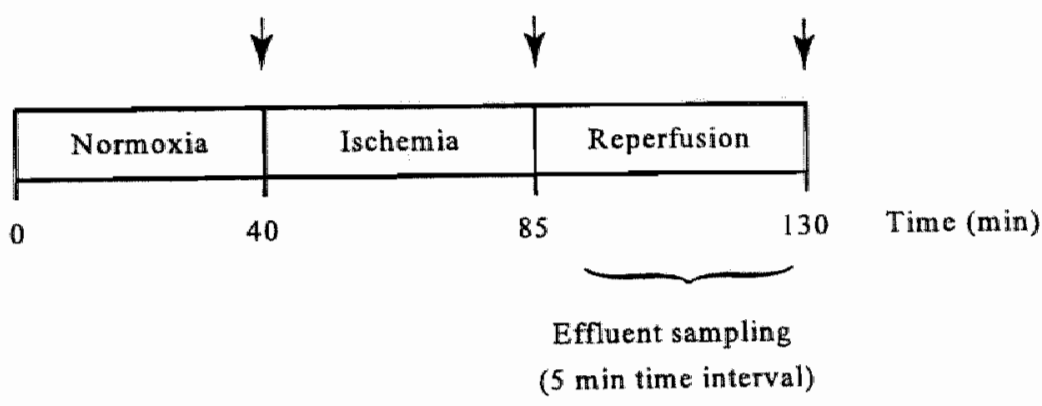

Figure 7.2 Schematic representation of the perfision protocol for normoxic, ischemic and reperfused rat hearts. Tissue biopsies were aken at the end of each stage, indicated by arrows in the figure. Left wentricular biopstes taken from andesthetized open-chested animals senwed as controls. Effhuent samples were only collected dwring the reperfusion period. For stabilization purposes during both normoxic and reperfusion periods antegrade perfusion was preacled by a short period of retrograde perfustow lasting $10 \mathrm{~min}$.

Following an initial stabilization period of $10 \mathrm{~min}$, during which the hearts were perfused retrogradely, isolated rat hearts were normoxically perfused in the antegrade way for $30 \mathrm{~min}$ (normoxic phase). Subsequently hearts were deprived from flow for $45 \mathrm{~min}$ (ischemic phase). Finally, flow was restored during the reperfusion period lasting $45 \mathrm{~min}$ (reperfusion phase). During reperfusion, just like the normoxic phase, the antegrade perfusion was preceded by an initial stabilization period lasting $10 \mathrm{~min}$. Left ventricular biopsies taken from anaesthetized, open-chested rats served as controls. Tissue biopsies were taken at the end of each stage (see Fig. 7.2). Coronary effluent samples were collected at $5 \mathrm{~min}$ time intervals for the assessment of lactate dehydrogenase $(\mathrm{LDH})$ release. To prevent the loss of enzymatic activity during 
storage an aliquot of $15 \%$ bovine serum albumin (BSA) was added to the effluent samples. These samples were immediately frozen in liquid nitrogen and stored at $-80^{\circ} \mathrm{C}$ for later biochemical analysis.

For each individual experimental condition, such as ischemia, three rat hearts were used. From each heart 2.3 transmural biopsies were taken from the left ventricular wall. For use in the proton microprobe relatively large biopsies with a volume of $1-2 \mathrm{~mm}^{3}$ are mounted on a piece of cork using Tissue-tek (Miles, Elkhart, USA). The biopsies were very rapidly submersed in liquid isopentane chilled by liquid nitrogen. Subsequently cryosections were cut at $-20{ }^{\circ} \mathrm{C}$ at a thickness of $10 \mu \mathrm{m}$ in a cryostat microtome (Anglia Scientific AS600, Cambridge, England). For electron microscopy, biopsies were cut into smaller pieces and were

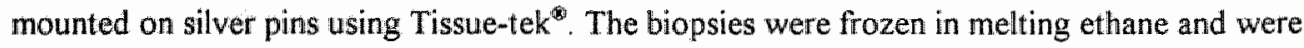
cut at a thickness of 100-150 nm using a cryo-microtome (LKB cryo-nova, Bromma, Sweden).

From the results presented in Chapter 5 it followed that some extra measures should be taken to prevent rehydration of freeze-dried cryosections in order to reduce the possible effects of rehydration, such as redistribution of diffusable elements. For this reason in the present study the freeze-dried sections the samples were stored under vacuum during transportation. Following transportation the sections were positioned in the target wheel of the proton microprobe target chamber, which was evacuated immediately thereafter.

\section{Lactate dehydrogenase (LDH) release from the isolated hearts during the reperfusion period}

The LDH release from the isolated rat hearts during the reperfusion period was determined earlier by Cornelussen [COR96]. Since the perfusion set-up, animals and experimental procedures used were exactly the same, the LDH release data were taken from this study.

\section{Histological amalysis}

For analysis in the proton microprobe sections were cut at a thickness of $10 \mu \mathrm{m}$. In order to allow visual inspection of these sections during sectioning each alternate section was stained with toluidine blue. The unstained sections were used for PIXE microanalysis, whereas the toluidine blue stained sections were analyzed by light microscopy in order to find out whether the individual cells are clearly visible. The light microscope pictures were used for a rapid selection of the region of interest to be analyzed by the proton beam on unstained adjacent sections.

\section{Proton microprobe analysis}

The region of interest on the freeze-dried cryosection could be selected within a few minutes using light microscope pictures of stained adjacent sections (see above). Subsequently, prior to quantitative X-ray microanalysis STIM was used for localization of individual cells. During both STIM and PIXE analysis an area of about $90 \mu \mathrm{m} \times 90 \mu \mathrm{m}$ was covered by the scanning proton beam. The experimental conditions under which these experiments were performed, such as beam dimensions, irradiation time per sample, scan frequency, etc, are described in Chapter 6. 
To investigate cellular heterogeneity it is necessary that different cells within a tissue section can be recognized, distinguished and separately analyzed by the scanning proton beam. To date the control system of the scan magnet of the Eindhoven microprobe only provides the possibility to perform a rectangular scan. It therefore is not possible to select any arbitrary area, for instance exactly corresponding with an individual cell, to be analyzed by the scanning proton beam. As a result on-line analysis of single cells is not possible. It is important to note that within an area of $90 \mu \mathrm{m} \times 90 \mu \mathrm{m}$ scanned by the proton beam several myocardial cells (generally about $4-5$ cells) and some extracellular space next to these cells were visible. During off-line data analysis regions of interest within the area scanned by the proton beam, corresponding with myocardial cells $s_{n}$ could be selected.

From the 2-3 biopsies taken from one individual heart sections stained with toluidine blue were analyzed by light microscopy (see above: Histological analysis) to select a biopsy from which the sections contained myocardial cells that could easily be recognized and distinguished from each other. Subsequently, from this biopsy 10 sections were cut which were alternately stained and thus 5 unstained sections were available for proton microprobe analysis. In practice from these sections one or two sections were analyzed in the proton microprobe, whereas the remaining sections served as a "backup" in case sections were lost during freezedrying or subsequent transportation. For each section one or two areas of about $90 \mu \mathrm{m} \times 90 \mu \mathrm{m}$ were analyzed by the scanning proton beam. Taking into account that within this scanning area about $4-5$ individual cells are visible it thus follows that for each experimental condition imposed on the rat hearts about 20-50 individual cells, originating from 3 hearts, were analyzed.

\section{Electron microprobe analysis}

Ultrathin sections (50-150 nm) from the same biopsies used for proton microprobe analysis were analyzed in a Philips CM-12 electron microscope. For a more extensive description on the sample handling and experimental conditions the reader is referred to Section 5.1 and Section 6.1. The CM-12 electron microscope was used for both analysis of tissue ultrastructure and quantitative X-ray microanalysis. Since the quantitative electron microprobe experiments were performed principally to confirm some findings from proton microprobe analysis, for each individual experimental condition only about 6-10 spots on one or two sections originating from one or two hearts were analyzed.

\subsection{Results}

\section{$L D H$ release during perfision experiments}

During previous perfusion experiments Cornelussen [COR96] determined the LDH release from reperfused rat hearts. These experiments were performed under the same conditions as described earlier in this thesis (see Chapter 5). He found that during reperfusion (duration $45 \mathrm{~min}$ ) following a period of $45 \mathrm{~min}$ of global ischemia the average total LDH release amounted to $16.6 \pm 3.8 \mathrm{ULDH}$ per gram wet weight. Taking into account that a rat heart of the same age contains about $314 \mathrm{U} \mathrm{LDH}^{-{ }^{-1}}$ wet weight [VOR93] it follows that only about 
$5.5 \%$ of all myocardial cells have lost their LDH content during reperfusion and thus have been damaged irreversibly.

\section{Histological analysis}

It appeared that with respect to histology no appreciable differences between the control, normoxic, ischemic and reperfused rat heart tissue became manifest (data not shown). In contrast with tissue from hearts subjected to the calcium paradox experiments (see Chapter 6), even after ischemia followed by reperfusion no substantial damage to the cell structure could be observed by visual inspection. It thus appears that, despite the fact that from the assessment of the LDH release from the heart it can be concluded that about $5.5 \%$ of the myocardial cells is damaged irreversibly, these damaged cells were not identified by visual inspection of the tissue sections.

\section{Proton microprobe results}

\section{a. Localization of individual cells using STIM}

For fast localization of individual cells within a region of interest of a given tissue section STIM was used in combination with stained adjacent sections. Figure 7.3 shows a typical result of both the STIM analysis of an unstained tissue section as well as the light microscope analysis of the toluidine blue stained adjacent section. The thickness at which the sections were cut $(10 \mu \mathrm{m})$ is in the same order of magnitude as the myocardial cell thickness (10 to $20 \mu \mathrm{m}$ ).

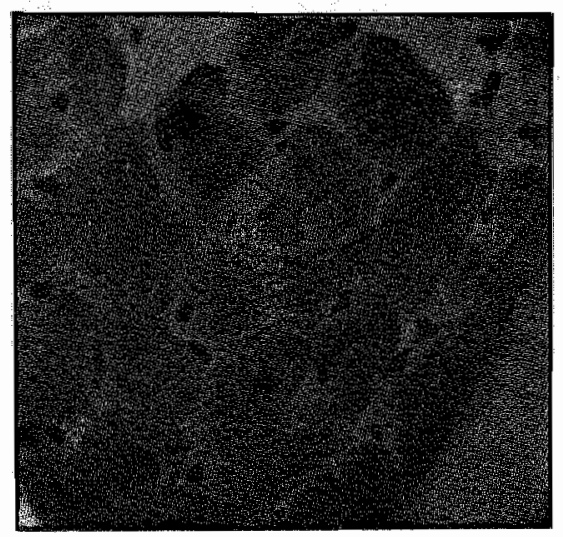

a

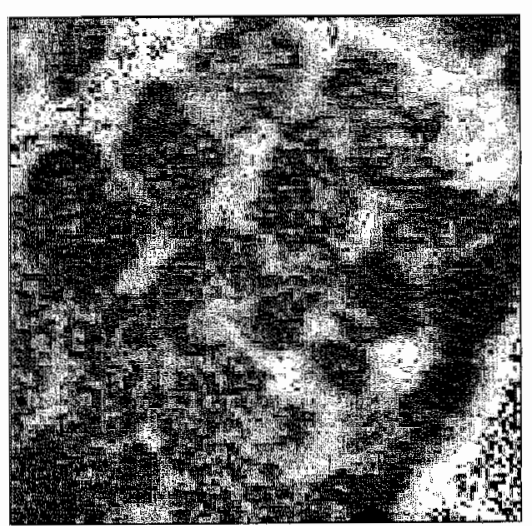

$\mathrm{b}$

Figure 7.3 Light microscopy picture of a staned myocardial rissue section (a) and STMM picture of an unstained adjacent section (b). Sections were cut at 10 um thickness. The area scanned by the proton beam was about $90 \mu \mathrm{m} \times 90 \mu \mathrm{m}$. Note the strong similarity between the two pictures with respect to the cellular contours. 


\section{b. Qnomitative proton microprobe results}

Three rat hearts were used for each individual condition studied. From each heart 1-2 sections were analyzed. On each section at least one preselected area of $90 \mu \mathrm{m} \times 90 \mu \mathrm{m}$, in general containing about 4-5 individual myocardial cells, was analyzed by the scanning proton beam. Between 20-55 individual cells were selected and analyzed for each experimental condition (control: $n=20$; normoxic: $n=35$; ischemic: $n=23$; reperfused: $n=55$ cells). These cells were divided proportionally among the analyzed sections as much as possible, irrespective of the animal and heart used. The mean elemental content of single cells for control, normoxic, ischemic and reperfused rat heart tissue are given in Fig. 7.4.

In contrast with the preliminary results presented in Chapter 5 , in this study the values of the mean cellular chlorine $(\mathrm{Cl}$ ) content are also included. This is made possible by using an improved procedure to produce Formvar backing foils. In the present study cyclohexanon was used as a solvent instead of chloroform, which resulted in Formvar foils that were free of $\mathrm{Cl}$ contamination.

From comparison of the absolute values of the different conditions some alterations in mean cellular ellemental content become manifest (Table 7.1). It appears that the elemental content of cells analyzed in normoxic tissue deviates considerably from both control and ischemic tissue, whereas for most elements the differences in mean cellular content between control and ischemic tissue are less pronounced.

- The mean cellular $\mathrm{Na}$ content increases when normoxic tissue is compared with control tissue. This finding affirms the results presented in Chapter 5 . After 45 min of ischemia the $\mathrm{Na}$ content decreases again to a mean value of $-4,800 \mathrm{mg} \cdot \mathrm{kg}^{-1} \mathrm{dry}$ weight, whereas the mean $\mathrm{Na}$ content of the myocardial cells in reperfused tissue amounts to about $8,250 \mathrm{mg} \cdot \mathrm{kg}^{*}$ ${ }^{1} \mathrm{dry}$ weight, which is a significant increase in comparison with the Na content in ischemic cells.

- In comparison with control values the mean $\mathrm{Mg}$ content decreases for both normoxic and ischemic tissue to a mean content of about $750 \mathrm{mg} \cdot \mathrm{kg}^{-1}$ dry weight and shows a further declime after reperfusion. In reperfused tissue in four cells the $\mathrm{Mg}$ content had decreased to such an extent that the Mg peak in the PIXE spectrum did not exceed the Bremsstrahlungs background.

- For P no appreciable differences between the four conditions analyzed were observed with respect to mean cellular content. As will be shown further on in this chapter, for some pixels (corresponding with an area of about $3 \mu \mathrm{m} \times 3 \mu \mathrm{m}$ ) that lie within the cell boundaries high $P$ concentrations were found in reperfused tissue. It thus appears that local, intracellular $\mathbb{P}$ elevations might occur.

- For all experimental conditions the mean cellular $S$ content varies between about 13,000 to $16,000 \mathrm{mg} \cdot \mathrm{kg}^{-1} \mathrm{dry}$ weight.

- The $\mathrm{Cl}$ content increases slightly from control values of about $10,000 \mathrm{mg} \cdot \mathrm{kg}^{-s} \mathrm{dry}$ weight up to about $13,000 \mathrm{mg} \cdot \mathrm{kg}^{-1} \mathrm{dry}$ weight for ischemic tissue. Howewer, after reperfusion the mean cellular $\mathrm{Cl}$ content has increased about twofold up to about $25,000 \mathrm{mg} \mathrm{kg}^{-1} \mathrm{dry}$ weight in comparison with values from ischemic tissue. 

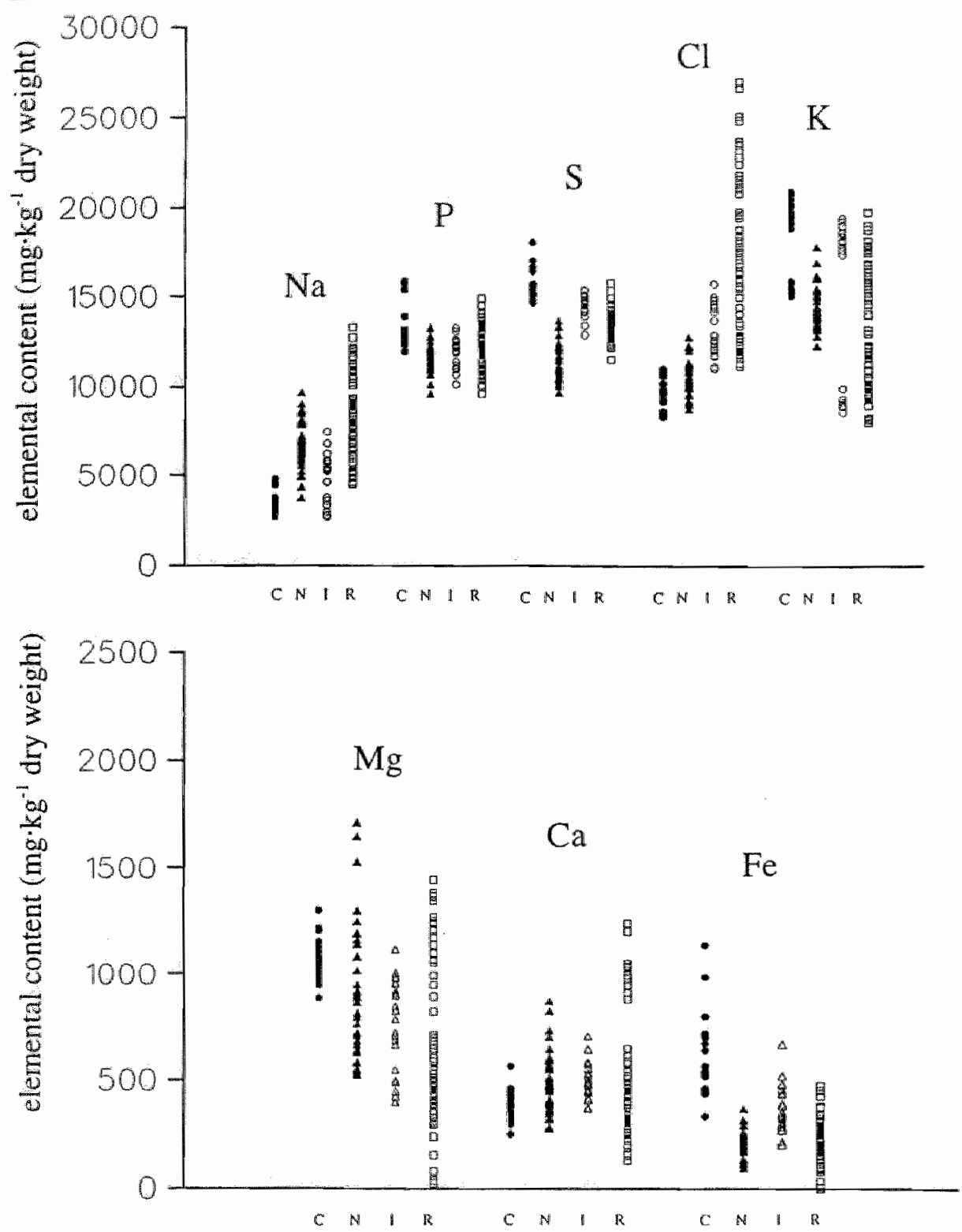

Figwre 7.4 Mean $\mathrm{Na}_{4}, \mathrm{Mg}_{\mathrm{g}}, \mathrm{P}, \mathrm{S}, \mathrm{Cl}, \mathrm{K}$. Ca and Fe content in $\mathrm{mg} \mathrm{kg}^{-1}$ dry weight of all cells analyzed in tissue from control ( $C$ ). normoxic $(N)$, ischemic $(I)$ and reperfirsed $(R)$ rat hearts (results obtained from proton microprobe analysis). Each point in the figure represenrs the mean elemental content of one individual cell. The mean elemental comtent was calculated according to method 2 as described in Chapter 6, i.e., after addition of the energy spectra of the pixels present within the boundaries of an individual cell. Note the large variation in mean cellular elemental content for reperfused rat bearts. 
- For potassium the opposite is true; after reperfusion $\mathrm{K}$ levels have decreased in comparison with the other experimental conditions. It should be noted, however, that in reperfused heart tissue about $20 \%$ of all analyzed cells contain less than $10,000 \mathrm{mg} \cdot \mathrm{kg}^{-1}$ dry weight, whereas for the other cells still values for the mean cellular $\mathrm{K}$ content are found that are comparable with the level of normoxic hearts. Also in ischemic tissue cells with low $\mathrm{K}$ content are found. This indicates that an appreciable heterogeneity in mean cellular content in response to $45 \mathrm{~min}$ of ischemia and subsequent reperfusion is observed. It should be noted that cells with comparable $\mathrm{K}^{*}$ contents were always found to be present in the same tissue section under investigation. Therefore the differences observed in mean cellular content always represent section-to-section variations

- The mean $\mathrm{Ca}$ content hardly alters when control, normoxic and ischemic tissue are compared. However, a considerable variation in the values for the $\mathrm{Ca}$ content is apparent. About $10-20 \%$ of the cells analyzed in reperfused heart tissue show an elevated $\mathrm{Ca}$ content after reperfusion. Just like $P$, it should be emphasized that in reperfused tissue very locally pixels were found with a $\mathrm{Ca}$ content that exceeded the mean cellular $\mathrm{Ca}$ content significantly.

\section{Variation in the mean cellular content within each stage analyzed}

It is apparent from Fig. 7.4 that the variation in the mean cellular content for normoxic and reperfused tissue is considerably higher than for both control and ischemic tissue. Especially after reperfusion the mean content of some elements, such as $\mathrm{Na}$ and $\mathrm{Ca}$, showed a considerable variation, ranging from values observed in control tissue to levels found in cells damaged due to the calcium paradox (see Chapter 6).

To obtain a more quantitative impression of the heterogeneity of the cellular content within each experimental condition, such as reperfused rat heart, in Table 7.1 the coefficient of variation, defined as (S.D. $/ \mathrm{X}$ ), is depicted (see also Chapter 6). It should be emphasized that this coefficient is dependent on both the statistical uncertainty in the determination of the mean cellular content and the variation in elemental content in case different cells are compared with each other.

The most striking result that follows from Table 7.1 is the increase in coeflicient of variation for almost all elements in reperfused tissue. It appears that not only those elements from which very low amounts are present in the sample, such as $\mathrm{Mg}$ and $\mathrm{Fe}$, show a high value for the coefficient of variation, but also for elements with high cellular content, such as $\mathrm{Na}, \mathrm{Cl}$ and $\mathrm{K}$, high values are found for reperfused rat heart tissue. It is also notable that for most elements analyzed, such as $\mathrm{Na}, \mathrm{Cl}$ and $\mathrm{Ca}$, the coefficient of variation for normoxic tissue is substantially higher than for both control and ischemic tissue. A possible explanation for this finding is that during the normoxic perfusion period the isolated hearts still need to adapt to the perfusion with the crystalloid buffer solution, resulting in a relatively large variation in the mean elemental content for most elements studied. In addition, the recovery period, i.e., the time between the attachment of the isolated heart to the aortic cannula and the moment that the heart starts to eject fluid, varies. This also might result in differences in values for the mean elemental content of the analyzed cells. At the end of the ischemic episode, lasting another 
$45 \mathrm{~min}$, the condition of the individual hearts might be more comparable, resulting in a lower value for the coefficient of variation.

Table 7.I Mean values \pm S.D. of the Na, Mg, P, S, Cl, K, Ca and Fe content of individual cells int control $(n=20)$, normoxic $(n=35)$, ischemic $(n=23)$ and reperfused $(n=55)$ rat heart tissue. For each experimental condition analyzed the coefficient of variation. defined as (S.D./X), is also shown.

\begin{tabular}{|c|c|c|c|c|}
\hline element & control & normoxic & ischemic & reperfused \\
\hline $\mathrm{Na}$ & $3,640 \pm 635$ & $6.755 \pm 1,315$ & $4,820 \pm 1,390$ & $8,440 \pm 2,395$ \\
\hline S.D. $/ X$ & 0.17 & 0.19 & 0.29 & 0.28 \\
\hline $\mathrm{Mg}$ & $1,080 \pm 100$ & $915 \pm 320$ & $765 \pm 215$ & $690 \pm 395$ \\
\hline S.D. $/ X$ & 0.09 & 0.35 & 0.28 & 0.57 \\
\hline $\mathrm{P}$ & $13,250 \pm 1,160$ & $11,550 \pm 680$ & $11,890 \pm 780$ & $12,335 \pm 1,155$ \\
\hline S.D. $/ \mathrm{X}$ & 0.09 & 0.06 & 0.07 & 0.09 \\
\hline $\mathrm{S}$ & $15,700 \pm 830$ & $11,200 \pm 1,065$ & $14,480 \pm \pm 560$ & $13.520 \pm 700$ \\
\hline S.D. $/ X$ & 0.05 & 0.10 & 0.114 & 0.05 \\
\hline $\mathrm{Cl}$ & $9,720 \pm 880$ & $10,440 \pm 950$ & $13,185 \pm 1,430$ & $16.760 \pm 4,245$ \\
\hline S.D. $/ \mathrm{X}$ & 0.09 & 0.09 & 0.11 & 0.25 \\
\hline $\mathrm{K}$ & $18.740 \pm 2,050$ & $14,445 \pm 1,240$ & $15,215 \pm 4.550$ & $13,160 \pm 3.290$ \\
\hline S.D. $/ X$ & 0.11 & 0.09 & 0.30 & 0.25 \\
\hline $\mathrm{Ca}$ & $375 \pm 70$ & $490 \pm \pm 145$ & $490 \pm 80$ & $50(1) \pm 275$ \\
\hline S.D. $/ K$ & 0.19 & 0.30 & 0.16 & 0.55 \\
\hline $\mathrm{Fe}^{*}$ & $620 \pm 195$ & $210 \pm 60$ & $360 \pm 110$ & $230 \pm 1100$ \\
\hline S.D.X & 0.31 & 0.29 & 0.31 & 0.43 \\
\hline
\end{tabular}

In conclusion it thus appears that, with respect to elemental concentration distribution, the tissue sections analyzed from reperfused tissue are heterogeneous. It is remarkable that this heterogeneity was never observed when cells within one region of interest irradiated by the proton beam, generally corresponding with an area of about $90 \mu \mathrm{m} \times 90 \mu \mathrm{m}$, corresponding with 4-5 cells, were compared. Heterogeneity in elemental concentration was only found by comparison of different tissue sections, sometimes originating from different rat hearts. 


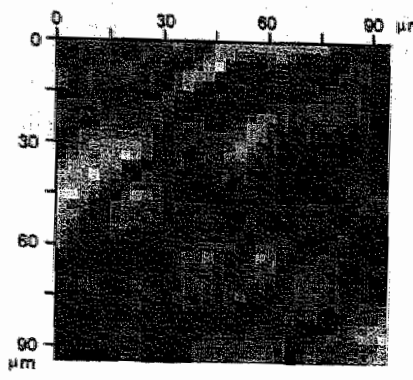

$\rho_{s} t$

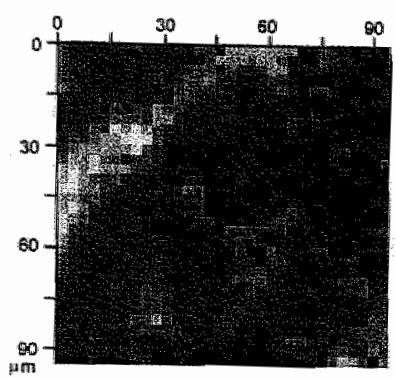

$\mathbf{P}$

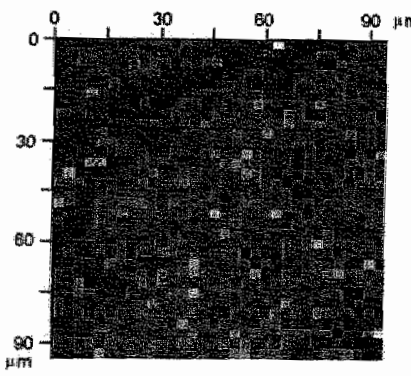

$\mathbb{K}$

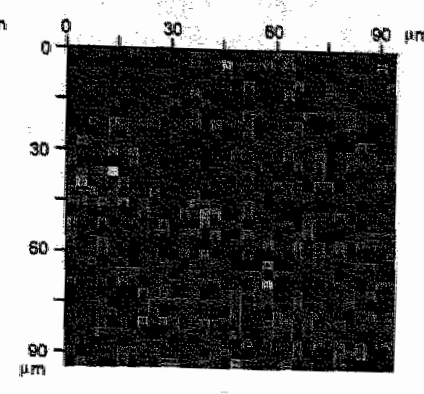

$\mathrm{Na}$

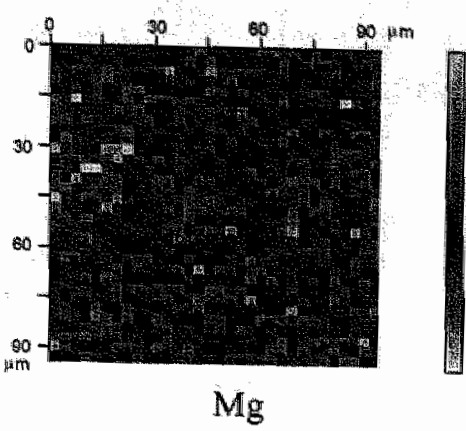

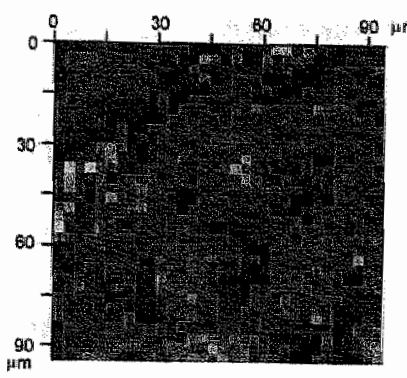

$\mathbf{S}$

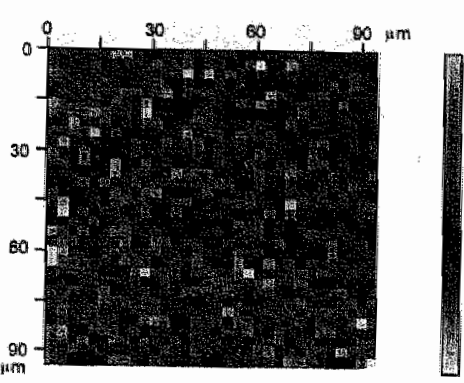

Cl

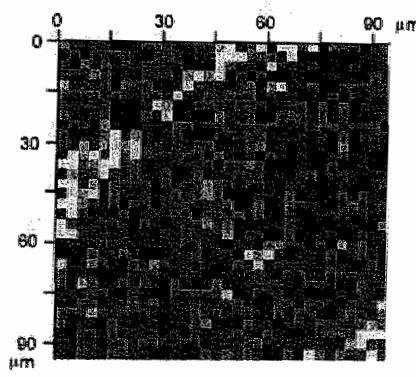

$\mathrm{Ca}$

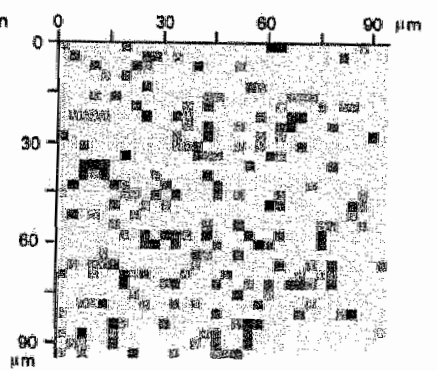

$\mathrm{Fe}$

Figure 7.5 Distribution of the $\mathrm{Na}, \mathrm{Mg}, \mathrm{P}, \mathrm{S}, \mathrm{Cl}, \mathrm{K}, \mathrm{Ca}$ and Fe content in reperfused rat heart tissue. In contrast with the determination of the mean cellular content (see Fig. 7.4). in this figure the elemental concentration is calculated for each position scamed by the proton beam (method $1_{*}$ Chaprer 6 , secrion 6.2$)$. The areal mass density $\left(\rho_{s} 1\right)$ distriburion was obtained from NBSNFS analysis and corresponds with the presence of freeze-dried tissue. One pixel in the figure corresponds with abou $3 \mu \mathrm{m} \times 3 \mu \mathrm{m}$. The total area covered by the scanning proton beam is about $90 \mu \mathrm{m} x 90 \mu \mathrm{m}$. 


\section{Local, intracellular differences in elemental concentration}

The mean cellular content of several elements, as depicted in both Fig. 7.4 and Table 7.1, was determined by analysis of the PIXE and NBS/NFS spectra that were obtained from the addition of imdividual spectra from each pixel present within one single cell (method 2 , see Section 6.2). By addition of the individual spectra better statistics are achieved and the resulting "sumspectra" are used to calculate the mean elemental content. As a result the information about the elemental content of each individual pixel, corresponding with an area of about $3 \mu \mathrm{m} \times 3 \mu \mathrm{m}$, is lost. This implies that the mean cellular content does not give an impression about local, intracellular heterogeneity. Therefore, in addition to the calculation of the mean cellular content, the elemental content has also been calculated for each position scanned by the proton beam (method 1). In contrast with the method as described above, in this case the energy spectra of the individual pixels are analyzed. This way differences in elemental content are determined at the level of the dimensions of the proton beam, i.e., about $3 \mu \mathrm{m} \times 3 \mu \mathrm{m}$, but with large statistical uncertainty.

A typical example of the distributions of the contents of the elements present in both normoxically perfused and reperfused rat heart tissue are displayed in Fig. 7.5. For most analyzed elements, such as $\mathrm{Na}, \mathrm{S}, \mathrm{Cl}$ and $\mathrm{K}$, no local variations within an individual cell are apparent for both normoxic and reperfused tissue. For these elements the highest contents are found at places corresponding with the highest areal mass density, i.e., the spots where freezedried material is present.

For both $\mathrm{Ca}$ and $\mathrm{P}$ in some instances locally elevated levels were observed in reperfused tissue, whereas no $\mathrm{Ca}$ and $\mathrm{P}$ "hot spots" were visible in normoxically perfused tissue. This finding is in agreement with earlier results (see Chapter 5). However, because of the use of the STIM technique in combination with PIXE and NBS / NFS analysis, now these locally high levels can be correlated with histological information obtained from STIM. As a result it is possible to delineate whether levels are elevated throughout the cell or intracellular differences exist. From Fig. 7.5 it appears that the latter is true, since only very locally few pixels show $\mathrm{Ca}$ and $P$ levels that strongly deviate from the values of surrounding positions.

It should be emphasized that Fig. 7.5 is a characteristic example of the result of proton microprobe analysis of reperfused rat heart tissue. In other cases the absolute content values of all positions within the area analyzed by the scanning proton beam agreed with levels found in control tissue and local, intracellular differences in elemental concentration could be attributed to the statistical uncertainty (generally between 10-20\%, see [MUN94]) in the determination of the elemental content at each position.

\section{Electron microprobe resulls}

From the proton microprobe analysis of reperfused tissue it followed that locally very high depositions of both $\mathrm{P}$ and $\mathrm{Ca}$ were found. However, the spatial resolution of the proton microprobe was not sufficient to identify precisely the subcellular localization of these "hot spots". Nevertheless, since it is known from literature [PIE95] that mitochondria might act as calcium scavengers during ischemia and reperfusion, it is tempting to state that these "hot spots" are located inside mitochondria. For this reason for a limited number of cases 
mitochondria and surrounding cytosol were analyzed in the electron microprobe to confirm Whis presumption. As a result it is of no statistical meaning to calculate the coefticient of variation for each condition analyzed in order to get an impression whether a heterogeneous response to the induced damage is observed. However, if the data for the different tissue sections analyzed by the electron microptobe are compared, some interesting tendencies became apparent (see Fig, 7.6).

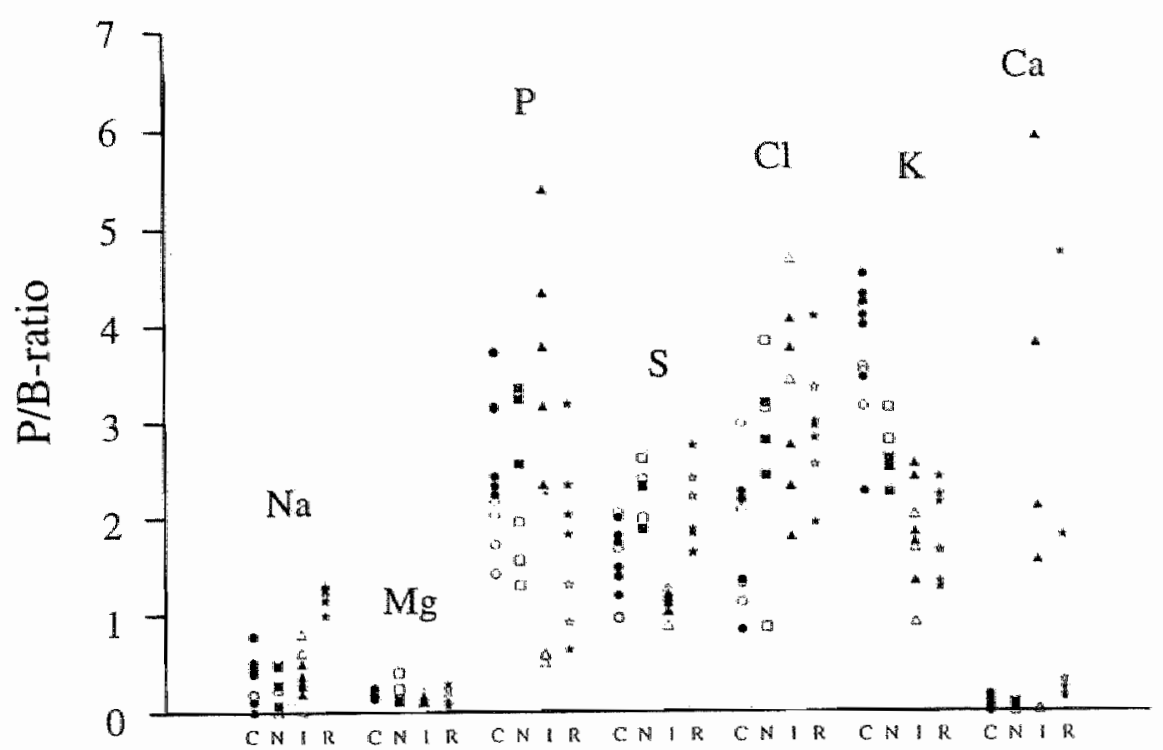

Figure 7.6 Results from EPMA analysis of mitochonaria (closed ploting characters) and cytoplasm (open plouting characters) in control (C), normoxic (N). ischemic (I) and reperfused (R) rat hearis. Each point in the figwre corresponds with an are of about $1, \mu m x$ I $\mu m$ scanwed by the electron beam. For all experimental condinons aboul 3-6 spots in both mitochondria and cytoplasm were andyzed. For each individual condition studied. such as ischemia, only a few sections (control:2; normoxic:l; ischemic: 1 ; reperfused:2), ariginaring from one rat heart, were anolyzed.

No appreciable alterations in elemental content of $\mathrm{Mg}, \mathrm{P}, \mathrm{S}$ and $\mathrm{Cl}$ in both cytoplasm and mitochondria are found between the different experimental conditions. On the other hand, the sodium concentration roughly remains unaltered for control, normoxic and ischemic hearts, but increases in both cytoplasm and mitochondria from about $2,000 \mathrm{mg} \cdot \mathrm{kg}^{-1}$ up to values of about $6,500 \mathrm{mg}^{-1} \mathrm{~kg}^{-1}$ dry weight after reperfusion. In comparison with control and normoxically perfused hearts the cytoplasmic and mitochondrial potassium content drops considerably for both ischemic and reperfused tissue. In this respect it is notable that no further decrease in $\mathrm{K}$ levels is observed when reperfused tissue is compared with ischemic tissue. 
For most elements no differences in elemental content were found between mitochondria and the surrounding cytoplasm. However a significant difference between cytoplasm and mitochondria is found for P. For all experimental conditions the mitochondrial phosphor content ig about 1.5-2 times higher than the cytoplasmic concentration. After ischemia and reperfusion also the calcum content of the mitochondria is strongly evated; calcium levels up to about $8,000 \mathrm{mg} \mathrm{Kg}^{-1}$ dry weight are found, while nomoxic Ca levels hardly exceeded the lowest detectable content. It should be noted that in reperfused tissue in some cases also for mitochondria very low Ca levels were found.

\subsection{Discussion}

The main objectives of this study were (i.) to determine the mean cellular $\mathrm{Na}, \mathrm{Mg}, \mathrm{P}, \mathrm{S}, \mathrm{Cl}, \mathrm{K}$, $\mathrm{Ca}$ and Fe content in control, normoxic and reperfused rat hearts and (ii.) to study whether cellular heterogeneity exists with respect to mean elemental content. For this reason the results of the proton microprobe analysis of both healthy and injured myocardial cells will be discussed first. Subsequently local intracellular differences in ellemental content will be treated.

In the previous chapter both morphological changes and alterations in elemental concentration in tissue severely damaged due to the calcium paradox manoeuvre were discussed. These results can be used as an indicator of the maximum alterations in elemental. content that are to be expected in rat hearts subjected to less damaging conditions, such as short-term ischemia followed by reperfusion. For this reason, in addition to the discussion of the physiological impact of the results obtained from the analysis of control, normoxic, ischemic and reperfused rat heart tissue, the results presented in this chapter will be compared with those from hearts subjected to calcium paradox experiments. Finally the results presented in this chapter will be compared with data from literature concerning the determination of the elemental content in normoxic, ischemic and reperfused hearts.

\section{Discussion of proton microprobe resulis}

a. Accuracy of determination of tissue conven us cellular heterogeneity

In the study of cellular heterogeneity with respect to the elemental content it should be emphasized that on the one hand experimental factors, such as the used sample preparation procedures, experimental methods and statistical uncertainty, and on the other hand (patho)physiological causes can have a significant influence on the measured alterations in elemental content.

In the previous section the coefficient of variation $(\mathrm{CV})$ has been introduced to give a numerical impression of the variation in the mean cellular elemental content. Specially in reperfused rat heart tissue large values for this coefficient of variation are found for most elements. For $\mathrm{Mg}$ and Fe, two elements from which very low amounts are present in the sample, this large value might be due to a large statistical uncertainty in the determination of the peak content in the PIXE spectrum. However, also for elements with a high cellular content, such as $\mathrm{Na}, \mathrm{Cl}$ and $\mathbb{K}$, high values for $\mathrm{CV}$ are found. In this case it is still possible that 
this high value is due to bad sample handling or the experimental procedures followed during proton probe analysis. However, this explanation does not fit the facts, since no large values for the coefficient of variation are found for control tissue ( $\mathrm{CV} \sim 10-20 \%)$. It thus can be concluded that high values for $\mathrm{CV}$ most likely reflect an appreciable heterogeneity in cellular content.

It also appeared that intercellular heterogeneity within one individual stage, such as reperfusion, was only found in case myocardial cells from different sections, sometimes originating from different hearts, were compared. Within a part of the tissue section analyzed by the scanning proton beam in one single run, corresponding with an area of about $90 \mu \mathrm{m} \times 90 \mu \mathrm{m}$, corresponding with 4 to 5 cells, no differences in mean cellular content were found in case different cells present within this area were compared. For this reason it is concluded that, within the region of interest analyzed by the scanning proton microbeam, adjacent myocardial cells in certain regions of the heart show altered comparable mean elemental contents, whereas cells in other parts of the myocardium were less affected. Accordingly it can be inferred that in ischemic and reperfused hearts within a relatively limited area analyzed by the proton beam either all cells are unaffected (or less affected) or all cells show a considerable imbalance in ion content.

\section{b. Difference benween control and normoxic tissue}

In general it is remarkable that for most elements the mean cellular content of normoxically perfused hearts differs considerably from control hearts. This result agrees with the preliminary results presented in Chapter 5. It also appeared that normoxically perfused rat hearts not only deviate from control hearts with respect to the absolute values of the elemental cellular content, but also the variation in those values is larger for normoxic hearts than in control hearts. It thus can be concluded that the perfusion of isolated rat hearts for $40 \mathrm{~min}$ with a Krebs-Henseleit buffer solution has a measurable impact on the elemental content values. It should be emphasized that differences in composition between blood and the crystalloid buffer solution exist, since the latter does not contain corpuscles and albumin. Therefore, a possible explanation for the deviation between control and normoxic hearts with respect to the mean cellular content is the adaptation of the cells to the altered composition of the extracellular space by the uptake or release of ions. Moreover, it is possible that the osmotic cell swelling that occurs during normoxic perfusion with the crystalloid buffer has a considerable effect on the distribution of diffusable elements. As discussed earlier, a possible explanation for the variation in the mean elemental content of the myocardial cells in normoxically perfused hearts is, next to the adaptation of the hearts to the normoxic perfusion, the variation in the time between the attachment of the isolated hearts to the aortic cannula and the moment the heart starts to eject fluid.

\section{c. Physiological implications of the proton microprobe reswlts and comparison with results from literature}

The main goal of our study was to assess the alterations in the elemental content of myocardial cells upon short-term ischemia and reperfusion and to investigate whether cellular 
heterogeneity exists. Moreover, the absolute values found for the elemental content were compared with results from literature. Specialiy when the present novel technique is explored to determine the elemental content of heart tissue such a comparison can give an impression of the reliability of the proton microprobe results presented in this chapter. However, it should be noted beforehand that in general large differences exist between the analytical techniques, experimental model (e.g., species of animals, perfusion protocol, etc) and sample preparation procedures used. Since all these factors can have a considerable impact on the absolute values that are obtained, it is emphasized that some caution should be exercised in case the values from different studies are compared in the following section.

- In ischemic tissue for most myocardial cells only a slight increase in Na content was observed. On the contrary, in reperfused tissue for many cells a significant increase in $\mathrm{Na}$ content did occur (about 2-3 fold increase compared with ischemic values), whereas in other cells the $\mathrm{Na}$ content was still comparable with control values. It seems probable that in the former case the ion homeostasis has been severely impaired due to the ischemic insult and the subsequent reperfusion period. In other myocardial cells, however, $\mathrm{Na}$ levels between these two extremes were found. The intermediate increase in intracellular $\mathrm{Na}$ can most likely be attributed to the $\mathrm{Na}^{*}-\mathrm{H}^{+}$exchanger, which actively transports protons out of the cell in exchange for $\mathrm{Na}^{+}$. Since ischemic cells become acidotic, this exchanger helps the cell to get rid of the excess protons by taking up sodium in exchange for hydrogen ions. Due to the relatively low intracellular $\mathrm{H}^{+}$concentrations this mechanism is much more subtle than the entry of sodium following rupture of the sarcolemma. According to Katz [KAT92], the sodium entry due to $\mathrm{Na}^{*}-\mathrm{H}^{+}$exchange is probably negligibly small because of the fact that the intracellular $\mathrm{H}^{+}$concentration is less than $1 \mu \mathrm{M}$, whereas the intracellular $\mathrm{Na}^{*}$ concentration amounts to about $12 \mathrm{mM}$. However, since the hydrogen ions are produced continuously during the ischemic episode, it seems likely that the effect of the $\mathrm{Na}^{+}-\mathrm{H}^{+}$exchanger on the intracellular $\mathrm{Na}^{+}$content is much more pronounced. In case the sarcolemma no longer can act as a barrier between the intra- and extracellular compartment a $\mathrm{Na}$ content of about $13,000 \mathrm{mg}^{-1} \mathrm{dr}^{-1}$ weight is to be expected (see also section 6.3). This value corresponds to a wet weight content of about $170 \mathrm{mmol} / \mathrm{l}$, which is in good agreement with the $\mathrm{Na}$ concentration of the crystalloid buffer solution $(160 \mathrm{mmol} / \mathrm{l})$.

Miller and Tormey [MIL95] employed electron probe microanalysis (EPMA) to study the subcellular redistribution of calcium and other electrolytes in myocardium subjected to global ischemia and reperfusion. In their study isolated ferret papillary muscles were exposed to ischemic conditions for $1 \mathrm{~h}$ and were subsequently reperfused for $5 \mathrm{~min}$. As Miller and Tormey assessed the mean elemental content of the whole "cell", i.e., a large, representative region of a cell encompassing myofibrils, mitochondria and the junctional sarcoplasmic reticulum, it is of special interest to compare their results with the present study. The most notable result from their study is the fact that the largest alterations in elemental content of the "cell" are found at the end of the ischemic episode and not after the reperfusion period. As compared with control in ischemic "cells" the Na content increased from 1,930 to $3,590 \mathrm{mg} \cdot \mathrm{kg}^{-1} \mathrm{dry}$ weight. At the end of the reperfusion periad a $\mathrm{Na}$ content of the "cell" of $3,840 \mathrm{mg} \cdot \mathrm{kg}^{-1} \mathrm{dry}$ weight was found in their study. It thus appears that in 
addition to a difference between the absolute values for the cellular Na content found in both studies, also a difference exists with respect to the moment the largest alterations in cellular content did occur, i.e., at the end of the ischemic or the reperfusion period. A possible explanation for these discrepancies, next to the apparent differences between the experimental models used in both studies, is the fact that in the study of Miller and Tormey for reperfused cells only data from moderately injured cells were given and data from severely injured cells, who had most likely an electrolyte profile similar to extracellular fluid, were excluded.

Sasaki et al. [SAS94] performed EPMA analysis on human, right atrial wall biopsies obtained during coronary artery bypass surgery at non-ischemia (before aorta-clamping), ischemia and reperfusion periods. It is important to note that, in contrast with the perfusion protocol used in the study described in this chapter, the patients were receiving surgery with hypothermic global ischemia. This means that the myocardial temperature was kept around $14^{\circ} \mathrm{C}$ during ischemia in order to decrease the activity of enzymes and thus reduce the injury due to the ischemic insult. Nevertheless, at the end of the ischemic period the $\mathrm{Na}$ content had increased from $1,000 \mathrm{mg} \cdot \mathrm{kg}^{-1} \mathrm{dry}$ weight in non-ischemic tissue up to $3,450 \mathrm{mg} \cdot \mathrm{kg}^{-1} \mathrm{dry}$ weight after ischemia. At the end of the reperfusion period a further increase in $\mathrm{Na}$ content up to $5,100 \mathrm{mg} \cdot \mathrm{kg}^{-1}$ dry weight was observed.

The results of Sasaki et al show close resemblance to the findings of Liu and colleagues [LIU93], who studied the myocardial $\mathrm{Na}, \mathrm{Mg}, \mathrm{K}$ and $\mathrm{Ca}$ content in rat hearts using atomic absorption spectroscopy (AAS). They found that after 35 min of ischemia the $\mathrm{Na}$ content had increased from 1,250 up to $2,830 \mathrm{mg} \cdot \mathrm{kg}^{-1}$ dry weight. At the end of a subsequent period of reperfusion, lasting $60 \mathrm{~min}$, a Na content of $3,620 \mathrm{mg} \cdot \mathrm{kg}^{-1} \mathrm{dry}$ weight was observed. This intracellular increase in $\mathrm{Na}$ content was also found by Ishibashi and coworkers [ISH93] in isolated rat hearts during $1 \mathrm{~h}$ of ischemia. However, in their ${ }^{23} \mathrm{Na}-$ NMR study no absolute values for the elemental content were given.

It therefore can be concluded that, in contrast with the results from Miller and Tormey, with respect to the alterations in mean cellular $\mathrm{Na}$ content our results agree reasonably well with the findings of Sasaki et al. [SAS94] and Liu et al. [LIU93]. However, it is notable that, despite the fact that the extent of the alterations found in both studies is comparable, the absolute values for the $\mathrm{Na}$ content found in our study are considerably higher for all conditions studied.

- For $\mathrm{Mg}$ a decrease in mean cellular content was observed after reperfusion. Since in some cases the Mg content of the cells had decreased to such an extent that the Mg peak in the PLXE spectrum did not exceed the background under the peak, it is obvious that part of the reperfused myocardium is damaged severely, thereby allowing magnesium to leave the cell. This result does not support the findings of Miller and Tormey [MIL95] (see also above). In their study no significant differences in $\mathrm{Mg}$ content were found between control and ischemic "cells". However, at the end of the reperfusion period an increase in the $\mathrm{Mg}$ content of the "cell" was observed. Their explanation for this remarkable finding is a possible magnesium influx resulting from $\mathrm{Na}^{+}-\mathrm{Mg}^{2+}$ exchange. However, Liu and coworkers [LIU93] did observe a two-fold decrease in $\mathrm{Mg}$ content after $60 \mathrm{~min}$ of reperfusion from 
800 down to about $420 \mathrm{mg}^{\circ} \mathrm{kg}^{-1} \mathrm{dry}$ weight. This post-ischemic magnesium release was also observed by Kirkels et al., who performed AAS analysis on the coronary effluent released during ischemia and reperfusion in isolated rat hearts [KIR89]. Another study from Thandroyen et al. [THA92], who employed EPMA to determine electrolyte alterations during progressive hypoxia lasting 1-5 hours, showed a decrease in the both the cytoplasmic and mitochondrial $\mathrm{Mg}$ content of cardiac myocytes during hypoxia as well.

From the above it follows that the results of the present study agree reasonably well with results from literature, except for the study of Miller and Tormey [MIL95]. In contrast with the results from the assessment of the $\mathrm{Na}$ content (see above) for $\mathrm{Mg}$ the absolute values found do not deviate appreciably from results obtained by others. A possible explanation for this effect is the fact that $\mathrm{Na}$, in contrast with $\mathrm{Mg}$, is a highly diffusable element [VIS85] and therefore is much more sensitive to differences in experimental conditions.

- It is interesting to note that, in contrast with calcium paradox results, no appreciable decline in $\mathrm{P}$ content was found. This element is present in both membranes, that contain large amounts of phospholipids, and in cytoplasmic energy-rich phosphates (ATP, GTP and phosphocreatine) and inorganic phosphates $\left(P_{i}\right)$. From the study of Van der Vusse and colleagues [VUS88] it is known that even in hearts subjected to the calcium paradox manoeuvre no substantial loss of phospholipids had taken place. Moreover, during ischemia the hydrolysis of both ATP and phosphocreatine liberates large amounts of inorganic phosphate. In reversibly damaged cells only a small fraction of this inorganic phosphate crosses the sarcolemma. Upon reperfusion inorganic phosphate is used again for the resynthsesis of ATP and phosphocreatine. Since the PIXE technique is based upon the ionization of individual atoms and thus no distinction can be made between free and bound atoms, it is expected that the total cellular $\mathrm{P}$ content has not changed considerably at the end of both the ischemic and the reperfusion period. Althougli no alterations in mean cellular $\mathbf{P}$ content were observect in ischemic and reperfused tissue, locally high $\mathrm{P}$ levels were found in reperfused tissue. Since these "hot spots" corresponded with locally elevated $\mathrm{Ca}$ levels and the dimensions of these spots only measured several micrometers, it seems likely that this accumulation can be attributed to the precipitation of calcium phosphate in the mitochondria. This notion is supported by our EPMA results (see also p. 117).

- Also for $\mathrm{S}$ no decline in mean cellular content was observed. Since this element is bound to proteins $\mathrm{S}$ can only leave the cell in case the cellular membrane has been heavily injured, giving rise to a massive release of cellular proteins. However, no histological changes could be observed in reperfused tissue and only about $5.5 \%$ of the total LDH (a cytosolic enzyme) content of the heart was released during restoration of flow. About $20 \%$ of the cellular proteins are cytoplasmic and only about $5.5 \%$ of the total cytoplasmic volume leaves the heart (as followed from the $\mathrm{LDH}$ release). Assuming that $\mathrm{S}$ is divided proportionally among the heart, this implies that only about $1 \%$ of the total cellular $\mathrm{S}$ was lost during ischemia/reperfusion. For this reason it is comprehensible that no considerable alterations in the mean cellular $\mathrm{S}$ content could be observed.

- The mean cellular $\mathrm{Cl}$ content gradually has increased from about $10,000 \mathrm{mg} \cdot \mathrm{kg}^{-1}$ dry weight 
for control tissue up to about $20,000 \mathrm{mg} \cdot \mathrm{kg}^{-1} \mathrm{dry}$ weight after reperfusion. If this value is converted into a wet weight concentration a value of $169 \mathrm{mmol} / 1$ is found. Just like the results found for hearts subjected to the calcium paradox, this value exceeds the $\mathrm{Cl}$ concentration in the crystalloid buffer solution, which amounts to $142.2 \mathrm{mmol}$. A possible explanation for this observation is the binding of this anion to positively charged proteins within the intracellular compartment, resulting in an intracellular accumulation of $\mathrm{Cl}$.

Just like the results of the present study Sasaki et al. [SAS94] observed a two-fold increase in $\mathrm{Cl}$ content after reperfusion. After $5 \mathrm{~h}$ of hypoxia Thandroyen et al. [THA92] also found that the $\mathrm{Cl}$ content had increased two-fold from 8,200 up to $16,200 \mathrm{mg} \mathrm{kg}^{-1} \mathrm{dry}$ weight. Upon ischemia/reperfusion Miller and Tormey [ML95] observed a less pronounced increase in $\mathrm{Cl}$ levels from about 2,850 up to $3,900 \mathrm{mg}^{-\mathrm{kg}^{-1}}$ dry weight. It thus. appears that the tendencies observed in all these studies show close analogy, although significant differences in absolute values for the $\mathrm{Cl}$ content are found.

- The decrease in $\mathrm{K}$ content due to ischemia followed by a period of reperfusion is observed in many other studies [SAS94, THA92, ISH93, LIU93, HAG90]. However, some important differences between the results obtained by other investigators exist. First, it is notable that some authors only find a dramatic decrease in $\mathrm{K}$ content at the end of the ischemic episode and no further decline in cellular $\mathrm{K}$ levels during subsequent reperfusion [SAS94], whereas others [LIU93] observe a significant decrease in $\mathrm{K}$ content during reperfusion as well. Miller and Tormey [MIL95] even observed a slight increase in cellular $\mathrm{K}$ dry weight content at the end of the reperfusion period. However, according to the authors this slight increase in [K] per dry mass actually corresponds to a decrease in aqueous concentration in the order of 20-30\% due to water accompanied by $\mathrm{Na}$ and $\mathrm{Cl}$ and a consequent increase in cell volume. Despite these differences it appears that the absolute values for the pre-ischemic and post-ischemic $\mathrm{K}$ content found in the studies mentioned above do mot deviate considerably.

- Although the increase in mean cellular $\mathrm{Ca}$ content after reperfusion is substantial as compared with control, normoxic and ischemic tissue, the highest $\mathrm{Ca}$ values are found very locally within the intracellular compartment. Just like $P$, it is very likely that these "hot spots" correspond with the presence of mitochondria. The presence of calcium in the mitochondria due to ischemia and hypoxia has been demonstrated by many authors [ALL90, HAG90, THA92, SAS94]. However, recent results of Miller and Tormey [MIL 95] showed that calcium accumulated in the mitachondria during ischemia was released again upon reperfusion. They concluded that the sarcoplasmic reticulum in reperfused cells may have helped to maintain the intracellular $\mathrm{Ca}^{2+}$ levels at low values by storing calcium released from the mitochondria. It is emphasized again (see above) that these results only refer to moderately injured cells and that cells that showed signs of irreversible damage were not included in their study.

\section{d. Comparison with reswlts from hearts subjected to calchum paradox experiments}

The absolute Na values in some cells in reperfused tissue of about $13,000 \mathrm{mg}^{\mathrm{kg}} \mathrm{kg}^{-1} \mathrm{dry}$ weight correspond with the values found in heart tissue subjected to the calcium paradox (see 
Chapter 6). As was shown in the previous chapter, this value agrees with the Na concentration in the crystalloid buffer solution, which is to be expected when the cellular membrane no longer is a barrier between the intra- and extracellular compartment. For other cells, however, sodium levels were found that were still at the level of control tissue. The same finding holds for chlorine. However, for the other elements analyzed the alterations are less dramatic in comparison with the results from calcium paradox experiments and all values for the mean cellular content differ from those after the calcium paradox. The lowest $K$ levels found in reperfused tissue amounted to about $8,000 \mathrm{mg}^{1 / 2} \mathrm{~kg}^{-1} \mathrm{dry}$ weight, whereas in tissue sections from hearts subjected to the calcium paradox values down to $2,000-3,000 \mathrm{mg}^{-1} \mathrm{~kg}^{-1} \mathrm{dry}$ weight were observed. Moreover, in contrast with the calcium paradox results, for some elements, such as $\mathrm{P}$ and $\mathrm{S}$, no substantial changes were observed after ischemia and reperfusion.

In conclusion it can be stated that for most elements the tendencies observed in the alterations in mean cellular content agree with the results from the analysis of hearts subjected to the calcium paradox experiments. It thus seems that, with respect to the mean cellular content, the damage inflicted upon the hearts during ischemia and reperfusion is less severe than during the calcium paradox manoeuvre. This finding is supported by the observation that the coefficient of variation for the elemental content after reperfusion is much higher in comparison with calcium paradox results. Moreover, the LDH release from the reperfused hearts (about $5.5 \%$ of the total LDH content of the heart) was very low, whereas after calcium paradox at least $70-90 \%$ of all cells had lost their LDH content. The former result implies that, in contrast with the hearts subjected to the calcium paradox manoeuvre, after ischemia and subsequent reperfusion within an individual heart the ionic imbalance created varies extensively. For this reason it is expected that upon ischemia/reperfusion an appreciable heterogeneity in mean elemental content of the myocardial cells exists.

\subsection{Summary and concluding remarks}

The most striking result from the studly described in this chapter is the considerable heterogeneity in mean cellular content in reperfused tissue. For some elements, such as potassium, this heterogeneity was also observed at the end of the ischemic period. In this respect it is important to note that the large differences between cells are not found within one single measurement with the scanning proton beam, covering an area of about $90 \mu \mathrm{m} \times 90 \mu \mathrm{m}$, i.e., large deviations are found only when cells from different tissue sections or even from different rat hearts are compared. This is not surprising, since it is known from other studies that some parts within the myocardial wall are damaged due to ischemia and reperfusion, whereas other parts remain unaffected [KAT92]. As was already discussed in the introductory chapter it is well known that in response to injury the endocardium is more sensitive to damage than the epicardial side. Due to this transmural heterogeneity, the small sample size and the fact that no attempts were made to determine the origin of the sample (endo- or epicardial side) the possibility exists that no alterations in mean cellular content are found in reperfused hearts, especially when the exact origin of the tissue sections used is unknown and the number of tissue biopsies taken from the heart is limited. 


\section{Chapter 8}

\section{General discussion}

\subsection{Introduction}

In this chapter first the results presented in the previous chapters are summarized. In addition the role of both the proton and electron microprobe in the analysis of healthy and damaged myocardial tissue sections and the (patho)-physiological significance of the results obtained using these techniques are discussed. Subsequently, a short synopsis of the problems that were encountered in the course of the present study is given. This is followed by a number of suggestions for further technological improvements and relevant physiological experiments to be performed in future.

\subsection{Summary and discussion of results}

The aim of the studies presented in this thesis was to explore the possibilities of the Eindhoven proton microprobe setup to study the alterations in elemental content of healthy and damaged rat hearts at the cellular level. The choice for the proton microprobe was partly based upon the promising results of other microprobe studies of biological materials [KIR89, MOR91]. From these studies it appeared that PIXE, when employed with a proton beam focused to a micronsized spot, yields quantitative elemental analysis with sensitivity at the $\mathrm{mg}^{\circ \mathrm{kg}^{-1}}$ level and identification of structural features at the microscale. This indicates that the proton microprobe enables one to determine low elemental contents at the cellular level. In general the alterations in elemental content in both healthy and damaged hearts can be studied either in isolated myocytes, within intact tissue sections or in whole tissue (bulk) preparations. The analysis of bulk tissue preparations precludes the determination of responses at the cellular and subcellular level. The use of isolated cells does not provide the possibility to study the response of cells in their natural environment. This is a major disadvantage, since it was already mentioned in Chapter 1 that a possible mechanism causing ischemic cell death is the ftacturing of sarcolemma by physical forces, such as the hypercontraction of neighbouring cells. For this reason in the present study proton microprobe analysis was applied to determine the elemental content of individual cells within tissue sections. In addition, the use of intact tissue allows to study the cell-to-cell differences in elemental content due to the ischemic insult (cellular heterogeneity). This is of special interest, since it is known that during the initial phase of ischemia a proportion of the cells is damaged irreversibly, whereas other cells are still viable.

Although the spatial resolution of the electron microscope is superior in comparison with the proton microprobe, the latter technique offers a better sensitivity (defined as the lowest detectable mass fraction of an element [JOH88]). This difference in sensitivity was substantiated by experimental determination of the limit of detection for calcium using both PIXE and EPMA (Chapter 3). In addition, this study showed that the PIXE procedures were sensitive enough to determine low amounts of calcium in the presence of much higher potassium levels, which is relevant in the analysis of myocardial tissue. 
In the course of the work described in this thesis several adaptations and improvements in experimental procedures and instrumentation of the Eindhoven proton microprobe have been introduced (Chapter 4). The most important improvement was the introduction of the STIM technique in order to provide the possibility to localize individual cells with the proton microprobe within freeze-dried cryosections of rat heart tissue. It was shown in Chapter 4 that this technique enables to localize myocardial cells with a spatial resolution of about $1 \mu \mathrm{m}$. Due to the very high detection efficiency of the STIM technique in practice only about 5 min of STIM analysis was sufficient to obtain histological information about the biological sample prior to quantitative PIXE and NBS/NFS analysis. In addition, comparison of the STIM pictures with light microscope pictures of toluidine-blue stained adjacent sections facilitated fast selection of a region of interest, e.g. an area containing clearly distinguishable myocardial cells, in the sample under investigation.

In order to ascertain whether it was possible to assess the tissue content of the elements of interest, viz, $\mathrm{Na}, \mathrm{Mg}, \mathrm{P}, \mathrm{S}, \mathrm{Cl}, \mathrm{K}, \mathrm{Ca}$ and $\mathrm{Fe}$, a preliminary study on control, normoxically perfused, ischemic and reperfused rat hearts was carried out (Chapter 5). In addition, this study was intended to gain insight into the possible artefacts introduced during the complete process of sample preparation. It appeared that the measured values for the elemental content agreed reasonably well with values from literature and also demonstrated that alterations in the tissue content of the elements mentioned above could be followed with a spatial resolution in the order of a few $\mu \mathrm{m}$. From the experiments discussed in Chapter 5 it was also inferred that two major improvements should be introduced to obtain more reliable results. In the first place, the STIM technique should be introduced in order to find and identify cellular structures. Secondly, these experiments showed that the freeze-dried sections should be treated with more caution to reduce the possible detrimental effects of rehydration on the (re)distribution of the elements of interest.

The calcium paradox manoeuvre is known as a condition leading to a high level of induced tissue damage [ZIM66]. The alterations in elemental content in hearts subjected to calcium paradox experiments, as assessed by proton microprobe analysis, were presented in Chapter 6 . The results of this extreme injury can be used as an indicator of the maximum changes in elemental content that are to be expected in case cardiac structures are subjected to less damaging conditions, such as short-term ischemia followed by reperfusion. The observation that 70-90\% of the total LDH content of the heart was released indicates that the cell membranes of the majority of cardiomyocytes had been damaged irreversibly during this manoeuvre (Chapter 6). As expected, proton microprobe analysis of tissue preparations from hearts subjected to calcium paradox experiments showed dramatic alterations in the elemental content of individual cell remnants. The fact that for most elements a small coefficient of variation was found $(<20 \%)$, when 32 individual cells originating from five hearts were analysed, suggests that all hearts were damaged to the same extent during the calcium paradox with respect to alterations in elemental content. At the end of the calcium paradox manoeuvre the sodium, chlorine and calcium content had increased dramatically. On the other hand, a significant decline in potassium, phosphor and sulfur levels was observed and in some cases the magnesium content had decreased to such an extent that this element could not be determined anymore.

As indicated above, during the preliminary proton microprobe analysis of control, normoxic, ischemic and reperfused rat hearts we were not able to distinguish individual myocardial cells 
within a tissue section (Chapter 5). However, the combined use of PIXE and STIM made it possible to determine the mean elemental content of individual cells in rat hearts subjected to short-term ischemia followed by reperfusion (Chapter 7). Comparison of the mean content of cells from reperfused rat hearts revealed that the cellular content of several elements, such as $\mathrm{Na}, \mathrm{Mg}$, $\mathrm{K}$ and $\mathrm{Ca}$, showed a considerable variation ranging from values observed in control tissue to levells found in cells damaged due to the calcium paradox. This heterogeneity in mean elemental content agrees reasonably well with the notion that only part of the cells has been damaged irreversibly upon ischemia/reperfusion, as only about $5 \%$ of all cells had lost their LDH content during reperfusion (Chapter 7). It is emphasized that heterogeneity in mean elemental content of different cells was only found in the case that myocardial cellis from different tissue sections, sometimes originating from different hearts, were compared. It thus appeared that within each individual region of interest, corresponding with an area of about $90 \mu \mathrm{m} \times 90 \mu \mathrm{m}$ containing on the average five individual cells, no intercellular heterogeneity was observed as analysed by the scanning proton beam. For this reason it is assumed that in certain regions of the heart clusters of neighbouring myocardial cells show an appreciable imbalance in ion content, whereas other parts of the heart contain cells that are less affected. This assumption is plausible, since it is known that for instance the endocardial side of the myocardial wall is more sensitive to ischemic damage than the epicardial side. However, in the present work no attempts were made to determine the orientation of the transmural biopsy taken from the left ventricular wall and therefore we are unable to pronounce upon the origin of the tissue sections analysed.

For ischemic and reperfused hearts as well as hearts subjected to the calcium paradox manoeuvre apart from differences in mean elemental content of individual cells also subcellular heterogeneity in elemental content was observed (Chapters 6 and 7). In some instances for both the calcium and phosphor content locally few pixels showed elevated values that strongly deviated from the surrounding pixels, corresponding with an area of several $\mu \mathrm{m}^{2}$. Additional electron microscope analysis of ultrathin sections from hearts subjected to ischemia, reperfusion and calcium paradox experiments confirmed that these "hot spots" were located in mitochondria, suggesting the precipitation of calcium phosphate in these organelles. This assumption is supported by the notion that the mitochondria act as calcium scavengers in situations of elevated cytoplasmic calcium levels [ZIM66, KAT92].

From the above it can be concluded that the preliminary results obtained in the course of the present study indicate that the Eindhoven proton microprobe offers the possibility to study alterations in elemental content at the cellular level. It should be emphasized that this study was intended to explore the possibilities of the Eindhoven proton microprobe to determine changes in elemental content in healthy and damaged hearts. Moreover, the number of hearts analysed in this study was limited. For this reason it is somewhat premature to use the present information on the cellular content of $\mathrm{Na}, \mathrm{Mg}, \mathrm{P}, \mathrm{S}, \mathrm{Cl}, \mathrm{K}$ and $\mathrm{Ca}$ and their possible mechanistic role in ischemia and reperfusion induced cardiac injury. Nevertheless, some interesting tendencies were observed during the proton microprobe analysis in tissue sections from hearts subjected to both ischemia/reperfusion and the calcium paradox manoeuvre.

First, comparison of the mean content of cells within one individual stage, such as ischemia, revealed that appreciable differences in mean cellular content of the elements of interest were 
apparent. In reperfused tissue for many cells the $\mathrm{Na}$ content had increased 2-3 fold in comparison with hearts subjected to ischemia, whereas other cells showed $\mathrm{Na}$ levels that were still comparable with control values. Cells containing very high $\mathrm{Na}$ levels most likely suffered from severely impaired ion homeostasis. In other myocardial cells Na levels between these two extremes were found. As suggested in Chapter 7, this less pronounced increase in Na content is probably due to the exchange of extracellular $\mathrm{Na}^{*}$ with excess intracellular hydrogen ions present in the acidotic ischemic cells via the $\mathrm{Na}^{+}-\mathrm{H}^{+}$exchanger. At the end of the reperfusion period in some cells the $\mathrm{Mg}$ content had decreased dramatically. With respect to this element still some controversy exists and several authors report different findings, i.e., by some investigators even an increase in intracellular $\mathrm{Mg}$ after reperfusion was found [MLC5]. In consideration of the finding that the $\mathrm{Mg}$ content of cells can be very low after ischemia/reperfusion and the fact that this element is also found to have anti-ischemic properties; the proton microprobe seems a powerful tool to study the role of $\mathrm{Mg}$ in ischemic and reperfused hearts. In contrast with the hearts subjected to the calcium paradox manouvre, in ischemic and reperfused hearts no decline in $\mathrm{S}$ levels was observed. This result is comprehensible since calculations based upon the $\mathrm{LDH}$ release from the reperfused heart show that only about $1 \%$ of the total cellular $\mathrm{S}$ was lost during ischemia/reperfusion. The decrease in potassium levels after reperfusion as observed in most cells can probably be attributed to the efflux of this element from the intracellular compartment due to an impaired Na-K ATPase activity. Finally, the mean cellular calcium content had increased substantially at the end of the reperfusion episode.

\subsubsection{Comparison of proton and electron microprobe results}

In this study both the proton and electron microprobes were used for the assessment of alterations in elemental concentration. It should be realized that experimental differences between these techniques may result in absolute values that do not match exactly. In the present study both techniques are used complementary, since the proton microprobe has better sensitivity, whereas the electron microprobe has a better spatial resolution that facilitates to distinguish subcellular structures, such as mitochondria, but a lower sensitivity.

In general the same tendencies in alterations in elemental concentration were observed in both the electron and proton microprobe. However, some interesting differences exist. First, for some elements, such as $\mathrm{Ca}$ and $\mathrm{Fe}$, the amount of element present in the analysed tissue was too low to be detected with the electron microprobe. Only in the case of substantial alterations in the cellular content of elements such as in reperfused myocardium the presence of $\mathrm{Ca}$ within the mitochondria could be demonstrated. In control, normoxic and ischemic tissue the $\mathrm{Ca}$ concentration remained below the limit of detection. In addition no changes in $\mathrm{Mg}$ concentration at all were observed making use of the electron microprobe. On the contrary, changes in the content of the latter elements could be assessed with the proton microprobe. It should be realized, however, that because of the larger beam dimensions and the use of thicker tissue sections the proton microprobe gives a mean value of elements present within the volume irradiated by the proton beam, which might contain mitochondria, cytoplasm and part of the sarcoplasmic reticulum. 


\subsection{2 (Patho)-physiological significance of proton microprobe results}

On the basis of the proton microprobe analysis of control, normoxic, ischemic and reperfused rat heart tissue and tissue sections from hearts subjected to the calcium paradox manoeuvre, several conclusions with respect to the physiological implications of the alterations in elemental content could be drawn (Chapters 5,6 and 7). Firstly, from the agreement of the present results with those obtained by other groups the conclusion may be drawn that the results of the proton microprobe analysis are physiologically meaningful. This interpretation is supported by the fact that for some elements, such as phosphor, the observed alterations in the mean cellular content agreed reasonably well with calculations based upon results from literature. In addition, no large differences were found in case the mean elemental contents of cells from control hearts were compared with each other. This observation strongly supports the assumption that the heterogeneity in mean cellular content found in reperfused heart tissue can be attributed to pathophysiological causes instead of other factors that can have a significant influence on the measured alterations in elemental content, such as the used sample preparation procedures, experimental methods and statistical uncertainty.

From the synopsis of the results from proton and electron microprobe analysis it can be concluded that the combination of these techniques is a powerful tool to study ischemia and reperfusion induced changes in elemental content in cardiac tissue sections. However, the following items that were encountered in the course of the present study require more attention and/or need further improvement: $i$. the spatial resolution of the Eindhoven proton microprobe is not good enough to recognize subcellular organelles, such as the mitochondria. Furthermore, in some instances it still remains difficult to distinguish between the intra- and extracellular space, specially in case some overlap exists between different cells in a tissue section. $i$. the analysis time required to obtain statistically significant values for the local content of the various elements is considerably long (2-6 hr). As a result many experiments are needed to facilitate a physiologically meaningful comparison of the elemental content of a large number of cells with each other in order to check for cellular heterogeneity. In the following section several suggestions for further technical improvements and physiological experiments are given. Finally some clinical applications of the determination of the elemental content of cardiac tissue sections are presented.

\subsection{Future developments}

\subsubsection{Use of line scans}

From the above it can be concluded that in case high amounts of an element are to be determined at submicron scale (e.g. in intracellular organelles) the electron microscope compares favourably to the proton microprobe. It thus seems most profitable to use the proton microprobe either to determine the contents of elements which are not very abundant, such as $\mathrm{Mg}$ and $\mathrm{Fe}$, or to compare the mean elemental content of individual cells. In the latter case, however, the long measurement time required to analyze a large number of different cells is still a problem. A possible solution to this problem is the use of line scans. For example, when the proton beam is scanned linearly across the sample (e.g. using a scan pattern of $1 \times 1024$ pixels) instead of employing a rectangular scan area (e.g. $32 \times 32$ pixels) the same total number of positions is hit 
by the proton beam and thus the same analysis time is used. In this case more cells will be scanned by the proton beam at the expense of a reduction of the number of pixels per cell that are analyzed. Although in this case only about 5 pixels are used to calculate the mean elemental content of each individual myocardial cell a linear scan offers the possibility to compare many cells with each other and thus to study cellular heterogeneity more effectively. Moreover, the use of elongated scan patterns enables to compare cells originating from different parts of the heart, for instance the endo- and epicardial side of the left ventricular wall, within one single measurement. This seems a useful alternative, since the analyses presented in the present thesis showed that within one individual scan area, corresponding with about $90 \times 90 \mu \mathrm{m}^{2}$, no intercellular differences in mean content could be observed.

\subsubsection{Improvement of the detection efficiency}

There are several possibilities to realize an increase in the number of samples that can be analysed within a certain time span. First, a reduction of the measurement time per sample (or better sensitivity in the same measurement time) can be achieved by increasing the $\mathrm{X}$-ray detection efficiency. Watt demonstrated that the employment of a $45^{\circ}$ target geometry, i.e., the alignment of the sample at an angle of $45^{\circ}$ with respect to the beam axis and positioning of the $\mathrm{X}$-ray detector at $90^{\circ}$, allows a significant decrease in the distance between the target and the $X$-ray detector [WAT95]. In this way solid angles of $400 \mathrm{msr}$ can be achieved with a $60 \mathrm{~mm}^{2}$ detector, which is eight times the solid angle usually reached in the Eindhoven proton microprobe. This implies that an optimization of the X-ray detection efficiency would facilitate to analyse many more samples within the same measurement time. Also the beam time available can be increased when the small Isochronous Low Energy Cyclotron ILEC, an accelerator specially built to deliver $2.9 \mathrm{MeV}$ protons to the proton microprobe set-up, becomes fully available with appropriate running budget [HEI92].

\subsubsection{Improvement of the spatial resolution}

In the present study it was demonstrated that the combined use of PIXE and STIM provides the possibility to determine the mean elemental content of individual myocardial cells. However, although local subcellular elevations in content could be observed for some elements such as calcium and phosphor, it was not possible to identify subcellular structures. According to Mutsaers several measures can be taken to improve the spatial resolution of the Eindhoven proton microprobe [MUT95]. Due to the fact that the minimum obtainable spot size in general is limited by chromatic aberrations, a smaller beam spot size can be obtained by a reduction of either the beam energy spread, the beam divergence or the chromatic aberration coefficients themselves. Calculations on the influence of the chromatic aberrations on the beam spot size [MUT95] indicated that from these factors particularly reduction of the beam energy spread will result in a decrease in beam spot size down to about $1 \mu \mathrm{m}$ with a beam current of about $100 \mathrm{pA}$. The possible application of a Van de Graaff accelerator would reduce the aberrations because of the lower energy spread and higher brightness of this machine in comparison with the AVF-cyclotron of the Eindhoven University of Technology. The work of Legge and coworkers [LEG82] suggests that, in order to obtain submicron resolution, in addition to a reduction of the influence of 
chromatic aberrations also the influence of parasitic aberrations, such as stray electromagnetic fields and vibrations of the microprobe setup, should be minimized

From the about 40 proton microprobes that are in operation worldwide nowadays only few microprobe groups, among which the Melbourne group, can produce a beam with a dianeter smaller than $1 \mu \mathrm{m}$ and a beam current higher than $100 \mathrm{pA}$ [WAT81, LEG82, WAT95]. However, even if these specifications can be achieved, it remains difficult to obtain reliable results from proton microprobe analysis of subcellular components, such as mitochondria. First, the high beam current density will cause severe damage to the biological sample during analysis processes. Such damage can be observed as elemental and mass loss often associated with target deformation and shrinkage, which might produce a distorted image of the distribution of the elements of interest [CHO91, CHO95]. Therefore for biomedical purposes the Melbourne microprobe group deliberately employs a $1 \mu \mathrm{m}$ proton beam with a beam current of only $30 \mathrm{pA}$ in order to reduce beam induced damage and shrinkage of biomedical samples under investigation [CHO93]. Secondly, according to Lindh [LIN9S], who discussed the capabilities and problems of the proton microprobe in biology and medicine, identification of subcellular details is very difficult. In addition, specially in sections cut at a thickness of about $10 \mu \mathrm{m}$, these subcellular structures are covered by part of the cytoplasm. Consequently it is not possible to know how much other cell material, or even part of other organelles, is present within the volume irradiated by the proton beam. A possible solution to this problem would be the use of much thinner sections. However, this will result in an increase of measurement time or a decrease in sensitivity. Finally, it should be noted that it is very difficult to obtain submicron resolution combined with analytically meaningful beam current on a routine basis. This is mainly due to the fact that a decrease in beam spot size is accompanied by a reduction in beam current, resulting in longer measurement times or a lower sensitivity. It thus can be concluded that it seems advisable to exploit the possibility to study differences in mean elemental content of different myocardial cells, using about 5 pixels per cell to determine the mean celiular content, instead of trying to perform proton microprobe analysis on subcellular structures. In the latter case the technical problem to obtain smaller beam dimensions while preserwing a beam current of about $100 \mathrm{pA}$ seems too demanding. Moreover, it was mentioned above that a higher beam current density $\left(\mathrm{pA} / \mu \mathrm{m}^{2}\right)$ could result in a substantial increase in beam induced sample damage [CHO95].

\subsubsection{How to obtain statistically relevant results in future}

The long $(2-6 \mathrm{~h})$ measurement time needed to analyse an area of about $90 \mu \mathrm{m} \times 90 \mu \mathrm{m}, \mathrm{m}_{\mathrm{i}}$ combined with the fact that the Eindhoven cyclotron on average can deliver beam to the microprobe setup one day a week, puts large restrictions on the number of tissue sections that can be analysed. However, to obtain statistically significant results, an appreciable number of animals should be analysed. After $45 \mathrm{~min}$ of global ischemia followed by a reperfusion period lasting another $45 \mathrm{~min}_{\text {, }}$ about $5 \%$ of the myocardial cells had lost their LDH content and thus had been damaged irreversibly (Chapter 7 ). This implies that the probability to find injured cells is rather low in an arbitrarily chosen tissue section, originating from a transmural biopsy removed from the left ventricular wall. Therefore, if the analysed cells should be regarded as a true representation of the left ventricular wall, an appreciable number of cells $(n=100)$ is to be analysed to increase the 
probability that both injured and wable cells are analysed by the proton microprobe. Combined with the requirement to analyse at least 6-8 hearts for each individual condition studied, such as ischemia, and the fact that about 15 individual cells can be analysed at each day the cyclotron accelerator is at our disposal, it thus follows that the demands mentioned above can be met in about 140 days of proton microprobe analysis. Since the cyclotron is available to perform proton microprobe experiments only once a week, this implies that under the present circumstances about 3-4 years of additional research are requisite to study the required number of cells in control, normoxic, ischemic and reperfused cardiac tissue. Both the use of line scans (Section 8.2.1) and an improvement of the detection efficiency (Section 8.2.2) can significantly reduce the research time required.

\subsubsection{Use of independent markers to distinguish intra- and extracellular space and to recognize damaged cells}

In order to correlate the injury inflicted upon the cells with the alterations in elemental content measured by the proton microprobe, it would be very useful to have an independent marker that enables one to distinguish between irreversibly damaged myocardial cells, viable cells and cells that are mildly affected. In this respect the study of myocardial cells that are in the transition phase of the ischemic period, i.e., the phase during which the cells are still able to recover from the ischemic insult in case flow is restored, is of special interest. A recent study of Miller and Tormey [MIL95] shows that KCoEDTA can be employed to serve as an independent marker of damaged cells as well as a marker to discriminate between the intra- and extracellular space. In their study, after an initial perfusion with a bicarbonate buffer with a composition comparable with the KrebsHenseleit buffer used in our study, the perfusion solution was altered. The $6 \mathrm{mM} \mathrm{KCl}$ of the original buffer solution was substituted by $6 \mathrm{mM} \mathrm{KCoEDTA}$ and $5 \%$ dextran was added. CoEDTA ${ }^{-}$remains unbound in the extracellular fluid, it does not cross the membrane and has no known physiological effects [MIL93, TOR89]. As a result, the cobalt X-ray signal can serve as an extracellular fluid marker during electron probe analysis. Due to the fact that the diameter of the proton microprobe exceeds that of the (extracellular) space between two adjacent cells, the elemental content measured at the cell bounciaries might include significant contributions from the extracellular compartment. Quantification of the Co content and subsequent determination of the ratio of the content of each element to cobalt thus enables one to perform a cobalt correction technique [MIL93, TOR89] in order to assess the elemental content solely arising from the intracellular space. In addition, since cobalt cannot cross the membrane of a (healthy) myocardial cell, it also seems very likely that the presence of this element in the intracellular space can be used as an independent marker of irreversibly injured cells, since the sarcolemma of those cells no longer can act as a barrier between the intra- and extracellular compartment.

\subsubsection{The possible role of heavy metals in ischemia and reperfusion}

In the present studies the changes in the myocardial content of light elements, i.e., $\mathrm{Na}, \mathrm{Mg}, \mathrm{P}, \mathrm{S}$, $\mathrm{Cl}_{x} \mathrm{~K}$ and $\mathrm{Ca}$ was determined. However, recent studies suggest that also iron, copper, zinc and selenium are involved in several reactions leading to free radical production that partly may be responsible for the injury induced during reperfusion of the ischemic myocardium [POW94, 
PUC95]. In this respect it would be of interest to measure the tissue content of these elements under these conditions. As was already discussed in Chapter 2, the lowest detectable concentration of an element is determined by the background under the characteristic peak in the $\mathrm{X}$-ray spectrum. The bremsstrahlung radiation background produced during both proton and electron irradiation is comparable for low $\mathrm{X}$-ray energies. However, this background shows a dramatic decline in strength at higher energies for the proton microprobe, whereas this rapid fall in intensity with $\mathrm{X}$-ray energy is not observed for the electron beam. Consequently, specially for heavy metals, such as iron, copper, zinc and selenium, the sensitivity of the proton microprobe is much better in comparison with the electron microprobe.

\subsubsection{Application of the proton microprobe for clinical problems}

In the studies described in this thesis the changes in elemental content were determined after a period of global, zero-flow ischemia. According to Opie [OPI91] in cardiac diseases only parts of the buman myocardium become gradually deprived from oxygen and other substrates, particularly glucose and fatty acids, because of slowly forming coronary stenosis. In this respect the zero-flow model applied in our study may have low clinical relevance. However, during bypass surgery the heart is subjected to global ischemia, typically lasting 30-60 min. For this reason it is of great interest to gain insight in the morphological and elemental changes that are introduced during the ischemic episode. Moreover, because of the growing interest in performing coronary artery bypass surgery without the use of cardiopulmonary bypass $(\mathrm{CPB})$ and the rising importance of minimally invasive cardiac surgery [BOO97, HAR96] it might be valuable to compare the condition of the heart in prebypass and postbypass tissue biopsies for these different procedures. Currently, in animal studies transmural needle biopsy specimens taken from the apex of the ventricles before and 30 and 60 min after completion of cardiopulmonary bypass (CPB) are used for ultrastructural and histological examinations, usually performed in both light and electron microscope [BEN93, SCH96]. This way post mortem heart specimens can be qualitively evaluated for myocyte necrosis, intercellular or intracellular edema, mitochondrial and myofilament abnormalities, cell membrane disruption and microvascular damage. Proton microprobe analysis of left ventricular biopsies taken at the end of the ischemic episode might provide the surgeon with additional information about the darmage inflicted upon the heart during open heart surgery with or without $\mathrm{CPB}$ and during minimally invasive cardiac surgery. In general, however, such models should be carefully studied and evaluated in an animal model before clinical application becomes into scope.

In summary, in the present study the potentials to use the Eindhoven proton microprobe for the assessment of the elemental content of both healthy and damaged cardiac tissue were explored. From the agreement of the present resuits with those obtained by other groups it followed that the results of the proton microprobe analysis are physiologically meaningfull Moreover, although the number of specimens analyzed was limited, several conclusions with respect to the (patho)-physiological condition of control, normoxic, ischemic and reperfused rat heart tissue and of tissue sections from hearts subjected to the calcium paradox manoeuvre could be drawn.

Due to limitations in the beam spot size (about $3 \mu \mathrm{m} \times 3 \mu \mathrm{m}$ with a beam current of $100 \mathrm{pA}$ ) 
for the time being the quantitative analysis of intracellular structures remains very difficult. In some cases, however, intraceliular differences in elemental content ( $\mathrm{Ca}$ ) could be observed. It thus can be concluded that the Eindhoven proton microprobe is best suited to compare the mean elemental content of different cells in order to study cellular heterogeneity. The use of line scans and an increase in detection efficiency are possible improvements that allow a larger number of cells to be analyzed within the same analysis time.

In case some overlap of different cells or intra- and extracellular space in a tissue section exists, it is hard to "unravel" how the measured elemental content has been built up. This problem might be solved by the use of independent markers. In addition, use of these markers could also help to distinguish between irreversible damaged cells and cells that are still viable. This would be of great value, since this offers the passibility to study the relation between the condition of the cells and the elemental content as assessed by proton microprobe analysis. 


\section{References}

[ALL90] Allshire AP, Cobbold PH. 1990. Causes and effects of changes in cytosolic free calcium in the hypoxic myocardial cell. Pathophysiology of severe ischemic nyocardial injury, 297-314 (Piper HM, ed., Kluwer Academic Publishers, Dordnecht, The Netherlands)

[ALT79] Alto LE, Dhalla NS. 1979. Myocardial cation contents during induction of calcium paradox. Am Phys Society, H713-H719.

[AM193] Amirikas R, Jamieson DN, Dooley SP. 1992. Measurement of (p,p) elastic cross sections for $\mathrm{C}, \mathrm{O}$ and $\mathrm{Si}$ in the energy range 1.0-3.5 MeV. Nucl. Instr. and Meth. B77, 110-114.

[BEN93] Bench G, Saint A, Legge G.JF, Cholewa M. 1993. Applications of energy loss contrast strM. Nucli. Instr and Meth. B77, 175-183.

[BI L88] Bilsen van M, Vusse van der GJ, Snoeckx LHEH, Ants T, Coumans WA, Willernsen PHM, Reneman R. 1988. Effects of pyruvate on postischemic myocardial recovery at warious workloads. Pflugers Archiv-Eur J Physiol 413, 167-173.

[BER74] Bergmeyer HU, Bernt E 1994. UV assay for lactate dehydrogenase with pyruvate and NADH. Bergmeyer HU (ed) Methods of enzymatic analysis. Weinheim: Verlag Chemie GmbH, Vol 2: 574.579 .

[BER88]

[BIE91]

[BO097]

[BOR80]

[BRAOS]

[BU.186]

Bernards JA, Bouman LN. 1988. Fysiologie van de mens. Bohn, Scheltema \& Holkema Utrecht Antwerpen

Biersack JP, Ziegler JF. 1991. TRIM computer program, IBM, Yorktown Heights, New York.

Boonstra PW, Grandjean JG, Mariani MA. 1997. Reoperative coronary bypass grafling without cardiopulmonary bypass through a small thorwetony. Ann Thorac Surg 63, 405-407.

Borderie B. 1980. Present possibilities for bulk analysis in prompt gamma-ray spectrometry with charged projectiles. Nucl. Instr. and Meth. 175,465 .

Bragg WH, Kleeman R. 1905. Phil. Mag, 10, $\$ 318$.

Buja LM, Williams PK, Buja DK, Chien KR, Willerson IT. 1986. Comparative encets of cardiac myocyte injury induced by inhibition of the $\mathrm{Nat}^{*}-\mathrm{K}^{*}$ pump and intermediary metabolism. Clin Res 34,627A.

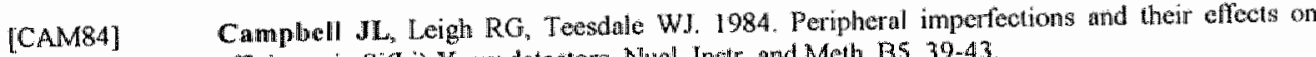
effieiency in Si(Li) X-ray detectors. Nucl. Instr. and Meth. B5, 39-43.

[CHA84] Chapman RA Rodrigo GC, Tunstafl $J$, Yales R., Busselen P. 1984. Calcium paradox of the heart: a role for intracellular sodium ions. Am Physiol Soc, H874.H879.

[CHA87] Chapman RA, Tunstall J. 1987. The calcium paradox of the heart. Prog. Biophys. Molec. Biol Vol $50,67-96$.

Chauvenet W. 1908. The general problems of spherical astronomy, the special applications to nautical astronomy, and the theory and use of fixed and portable astronomical instruments. 
[CHo91] Cholwa M. 1991. Changes in organic matcrials with scanning particle micobeams. Nucl. Instr. and Meth. $354,91.97$.

[CHO93] Cholewa $M$, Lege GIF, Weigold H, Holan G. 1993 . The use of a scanning proton microprobe in ADDG reserarch Mul. Instr: and Meth. B77, 282-286.

[CLOS5] Chollewa M. 1995. Summary of the workshop on micro-ion beam damaging. Nuel. Instr. and Meth. B104, 649

[CFU78] Chu WK, Mayer JW, Nicolet MA. 1978. Backscattering spectrometry. Academic Press New York.

[COH80] Cohen DD. 1980. A radially depiendent photopeak efficiency model for Si(Li) detectors. Wuct. Irstr. and Meth. 178, 481-490.

[COR96] Cornclussen $R$ 1996. Heat stess pretreatment in cardiac ischemia. PhD thesis, Maastricht Uiniversity.

[DHM76] Dempey GP, Bulliwant S. 1976. I. Microse. 106, 251.

[DOY97] Doyle BL 1997. Proceedings of the fifth intemational conterence on nuclear microprobe techtolology and applications. Albequerque, US.

[FRE82] Frederik PM. 1982. Cryoultramicrotomy-recognition of artifacts. Scan Electr. Micr., 709-721

[ERE81] Frederilk PM, Busing WM. 1981, I. Microse. 121, 191.

[FRE88] Frederik PM, Stuart MCA, Bomans PHH, Busing WM. 1988. Phospholipid, Nature's own slide and cover slip for cryo-electron microscopy. J. Microsc., wol 53, 81-92.

[GAN83] Gmote CE, Sims MA, Heide wan der RS. 1983. Mechnnisms of enzyme release in the calcium paradox. Eur Jleart J, 4 suppl. $H, 63-71$.

[GRO92] Groot ile MJM. 1992. The effect of lactate on the nomoxic, iscthemic and reperfused heart. Ph. D thesis, Manstrichl: University

[HAG90] Hagler HK. Buga LM. 1990. Subcellular calcim shils in ischemia and reperfusion. Pathophysiolowy of severe ischemic myocardial injury, 283-296. (Piper HM, ed, Kluwer Aondemic Publishers, Dordrecht, The Netherlands).

[HAN73] Hansen JS, MeGeorge JC, Nix D, Schimdi-Ot WD, Unus I, Fink RW. 1973. Accurate efficitncy calibration and properties of semiconductor detectors for low-energy puhotons. Nitcl. Instr. and Meth. 106, 365-379.

[HAR96] Harta RS. 1996. Minimally invasive heart surgery. Circullation 94, 2669-2670.

[HEI92] Heide wan der JA, Regt de RILJ, Gudden WAM, Magendans P, Hagedoorn HL, Mutsaers PHA, Mangnus AVG, Aendenromer AJR, Folter de LC, Voigt de MJA. 1992. A minicyclotron for a scantuing proton nicroprobe. Nucl. Instr: and Meth. B64, 336-34

[ISH90] Ishil K, Monita S. 1990. Int. Journal of PIXE vol. I No, 1, 1-29.

[ISH93] Ishibash T, Nakazawa M, Imai S. 1993. Ischemic changes in myocardial ionic contents of the isolated perfised rat hearts as studied by NMR. Mol Cell Biochemistry 119, 109-120 
[ACS4]

[JAN95]

[NEN83]

[JEN86]

[ $\mathrm{YOH70]}$

[JOH88]

[. $\mathrm{HOH} 122]$

[JYN95]

[KAT92]

[KTR89]

[KIT83]

[KRU92]

[KU191]

[LADS4]

[LAN1895]

[UEF87]

[LEG80]

[LEG82]

Jacobs R. 1994. NBS and NFS Analysis for Microbem Experiments: Master of Science thesis VDF/NK 94-28. Eindhoven Unwersity of Technology.

Jansen W. 1995. Bepaling enkele eigenschappen van een Si(Li)- en een LEGe-detector. Intermal report. WDF/NK 95-10. Eindhoven University of Technology.

Jennings R, Reimer K. 1983. Faciors involved in salvaiging ischemic myocardium: effect of reperfusion of arterial blood. Circ 68 (Suppl):1-25-136.

Jennings RB, Reimer KA and Steenbergen C. 1986. Myocardial ischemia revisited. The osmolar load, membrane damage and reperfusion. J Mol Cell Cardiol 18; 769-780.

Johansson TB, Akselsson R, Johansson SAE. 1970. X-ray analysis elemental trace unalysis at the $10^{-12} \mathrm{~g}$ level. Nucl. Instr. and Meth. 84,141 .

Johansson SAE, Campbell IL. 1988. PIXE: A novel technique for elemental analysis, Jotm Whiley \& Sons, Chichester.

Johansson SAE. 1992. Optimization of the sensitivity in PIXE analysis. Int. 1. of PLXE. Vol2. No.1, 33:46.

Jynge P, Falck $\mathrm{O}$. 1995. High magnesium improves the postischaemic recovery of cardino function. Cardiovascular Research, 29, 439.

Katz AM. 1992. Physiology of the heart. Raven Fress, New York.

Kirkels JH, van Echteid C.JA, Ruigrok TJC. 1989 intracellular magnesium during myocardial ischemia and reperfusion: possible consequences for postischemic recovery. I Mol Cell Cardiol $21,1209-1218$.

Kitazawa T, Shuman H, Somlyo AP. 1983. Quantitative electron probe analysis: problems and solutions. Ultransicroscopy $11,251-262$.

Krutzen PJG. 1995. Oorzaken van achtergrondstraling en de invloed hierop van experimentele parameters bij PIXE. Master of Science thesis. VDF/NK 92-94. Endhoven University of Technology.

Kuigen van WJP. 1991. Elementanalyse in hartweefsel. Master of Science thesis VDF/NK 91. 30. Eindhoven University of Technology.

LaDue JS, Wroblenski F "Karmen A. 1954. Serum gluntanic oxaloacetic transaminase activily in human acute transmural myocardial infarction. Science 120, 497-499.

Langendorf 0 . 1895 . Untersuchungen am übericbenden Satagetichtherzen. Pflagers Arch 61. $291-332$.

Lefewre HW, Schofield RMS, Overtey JC, MacDonald JD. 1987. Scanning transmission ion microscopy as it coplements particle induced $X$-ray emission microanalysis. Scanning Microscopy, Vol 1, No 3,879-889

Legge GJF, Mazzolini A.P. 1980. Elemental microanalysis of biological and medical specimens with a scanning proton microprobe. Nucl. Instr. and Meth. 168, $563-569$.

Legge G.JF, Jamieson DN, O'Brien PMJ, Mazzolini AP. 1982. Developments on the Mellbourne scanning proton microprobe. Nucl. Instr. and Meth. $149,663$. 
[LEG93] Legge GJF, Laird IS, Mason LM, Saint A, Cholewa M, Jamieson DN. 1993. High resolution imaging with high energy ion beams. Nucl. Instr. and Meth. B77, 153-168.

[LIN82] Linders PWJ, Stols ALH, Vorstenbosch wan de RA, Stadhouders AM. 1982. Mass determination of thin biological specimens for use in quantitative electron probe X-ray microunalysis. Scan. Elec. Mier. IV, 1603-16.15.

[LIN84] Linders PW. 1984. Mass delermination based on electron seattering in electron probe X-ray microandysis of thin biological specimens. Ph.D thesis.

[LIN95] Lindh 0. 1995. Cell biology, trace elements and nuclear microscopy. Nucl. Insitr. and Meth B104, 285-291.

[LIU93] Liu JX. Tarnonaka $K_{\text {, }}$ Yamamoto $K_{0}$ Takeo $S .1993$. Propafenone and disopyramide enhance post-ischemic contractile and metabolic recovery of perfused hearts. Eur J of Pharmacology, 250 , $361-369$.

[LLA90] Llabador Y, Bertault D, Gouillaud JC, Moretto Ph. 1990. Nucl. Irostr and Meth. B49, 435

[MAA96] Maanen wan IF, Mutsaers PHA, Verhoef BAW, de Voigt MJA. 1996. Scanning transmission ion microscopy (STIM) with a $3 \mathrm{MeV}$ proton cyclotron beam. Nucl. Instr and Meth. B113,382386 .

[MAE86] Manmhaut W, Vandenhaute J. 1986. Bull. Soc. Chim. Belg. 95, 407.

[MAJ96] Maj B, Mutsaers. PHA, Rokita E. 1996. Determination of the microbeam profile using deconvolution procedures. Nucl. Instr. and Meth. B113, 391-395

[MAU82] Maurel B, Amsel G, Nadai JP. 1982. Depth profiling with narrow resonances of nuclear reachions: theory and experimental use. Nucl. Instr. and Meth. 197, 1.

[MIL93] Miller TW, Tormey NMCD. 1993. Calciun displacement by lanthanum in subcellular compartments of rat ventricular myocytes: characterisation by electron probe microanalysis. Cardiovascular Research, 27,2106-2112.

[MIL95] Miller TW, Tomey JMeD. 1995. Subcellular calcium pools of ischaemic and reperfused myocardium characterised by electron probe Cardiovascular Research 29, 85-94

[MOR91] Moravec CS, Bond M. 1991. Calcium is released from the junctional sarcoplasmic reticulum during cardiac muscle contrastion. Am. Physiol. Soc., H989.H997.

[MUN94] Munnik F. 1994. Accurncy evaluation of absolute calibration in thick-target PIXE. Ph.D thesis, Eindhoven University of Technology.

[MUN94b] Munnik F. Mutsaers PHA, Rokita E, Vongt de MIA. 1994. Error propagation of the uncertainty in various quantities to the uncertainty in the primary PIXE yield and Secondary lo luorescence yield. Int, d. of PIXE, vol $3 / 2,145$.

[MUT95] Mutsaers PHA. 1995. Design and realisation of the Eindhoven scanning proton microprobe. Ph.D thesis, Eindhoven University of Technology.

[NAY81] Nayler WG, Grimwald PM. 1981. The effect of verapamil on calcium accumulation during the calcium paradox. J Mol Cell Cardiol $13,435-441$. 
[NAY84] Nayler WG, Perry SE, Elz IS, Daly MI. 1984, Calcium, Sodium, and the calcium paradox. Circ Res Vol.55, No.2, 227-237.

[OP191] Opie LH. 1991. The heart: Physiology and metabolism. 2nd edition Raven Press, New York.

[ORL89] Orlic I, Lenglet WJM, Vis RD. 1989. Accurate measurement of the relative Si(Li) detector efficiency for X-ray energies below $5 \mathrm{keV}$. Nucl. Instr. and Medh. A276, 202-209.

[OVE83] Overley JC, Connolly RC, Sieger GE, MacDonald JD, Lefeve HW, 1983. Energy-loss radiography with a scanning MeV-ion microprobe. Nuel. Instr, and Meth. 218,43-46.

[PIE95] Pierce GN, Caubryt MP. 1995. The contribution of ionie imbalance to ischemia/reperfusioninduced injury. I Mol Cell Cardiol 27, 53-63.

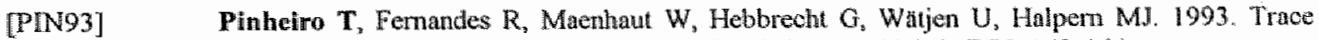
element changes in cardiovascular diseases. Nucl. Instr. and Meth. B75, 160-164.

[PIP90] Piper HM (ed). 1990. Pahophysiology of sewere ishemic myocardial injury. Kluwer Academic Publishers Dordrecht.

[POW94] Powell SR, Hall D, Aiuto L, Wapnir RA, Teichberg S, Tortolani AJ. 1994. Zine improves postischemic recovery of isolated rat hearts through inhibition of oxidative stress. Am Phys Society, H2497-H2507.

[PUC95]

Pucheu S, Coudray C, Vanzetto G. Favier A, Machecourt J, de Leiris J 1995. Time-course of changes in plasma levels of" trace elements after thrombolysis during the acute phase of myocardial infarction in humans. Biological Trace Element Research, Vol.47, 171-182.

[REI86] Reimer KA Jennings RB. 1986. Myocardial ischemia, hypoxia, and infarction. In: HA Fozzard E Haber, RB Jennings, AM Katz, HE Morgan (eds). The Heart and Cardiovascular System. Raven Press, New York, 1133-1201.

[ROO88]

[RUS81]

[RUT11]

[SAN73]

[SAS94]

[SCH88]

[SCH89]

[SCH96]

Roomans GM. 1988. Quantitative X-ray microanalysis of biological specimens. J. Electr. Micr. Techn. 9, 19-43.

Russell SB, Schulte CW, Fani S, Campbell JL. 1981. Annl. Chem, 53, 571.

Rutherforl E. 1911. Phill. Mag. 21, 669.

Sandwik GE, Hagedoorn HL, Schutte F. 1973. Nucl. Instr. and Meth. 106, 245.

Sasakl S, Sawada Y, Morita M, Hikita Y, Nakagaki I, Hori S, Sasaki S, Takeuchi A. 1994 Effects of a benzothiazepine calcium blocker on electrolyte alteration in human ischemic and reperfused myocardium. J Moll Cell Cardiol 26, 1037-1044.

Scherer J, Braun-Dullaeus K. U, Traxel K. 1988. Nucl Instr. and Meth. B30, 265

Schmidt RF, Thews G. 1989. Hurnan Physiology. Springer-Vertag, Berlin

Schwartz DS, Ribakove GH, Grossi EA, Stevens JH, Siegel LC, St. Gonr FG, Peters WS, McLoughlin D, Baumann FG, Colvin SB, Galloway AC. 1996. Minimally invasive cardiopulmonary bypass with cardioplegic arrest: a closed chest technique with equivalent myocardial protection. J Thorac Cardiovase Surg. 111, 556-566. 
[SEA83] Sealock RM, Mazzolini AP, Legge GJF, 1983. The use of He microbeams for light etement $X$ ray analysis of biological tissue. Wucl. Instr. and Meth. 218, 217-220.

[SLE92] Slezalk J, Tribulova $\mathbb{N}_{2}$ Ravingerova T, Singal PK. 1992. Myocardial heterogeneity and regional variations in response to injury. Laboratory Investigation, Vol.67, No.3, 322-330.

[SPE80] Speich M, Bousquet B, Nicolas G. 1980. Concentrations of magnesium, calcium, potassiurn, and sodium in human hear muscle after acute myocardial infarction. Clinical Chemistry, Vol. 26 , No.12, 1662-1665.

[SNO87] Snoeck LHEH. 1987. Ischemia tolerance of the hypertrophied rat heart. Ph.D. Thesis. Maastricht University:

[TEO93] Teo KK, Yusuf S. 1993. Role of magnesinum in reducing mortality in acute myocardial infarction. A review of the evidence. Drugs $46,347-359$.

[THA92] Thandroyen FT, Bellotto D, Katayama A, Hagler HK, Willerson JT, Buja LM. 1992. Subcellular electrolyte alterations during progressive hypoxia and following reoxygenation in isolated neonatal rat ventricular myocytes. Circulation Research Vol 71 , no 1, 106-119.

[TOR89] Tormey JMcD, Walsh LG. 1989. Strategics for spatial deconvolution of calcium stores in cardiac muscle. In: Russell PE, ed. Microbean analysis. San Francisco: San Francisco Press, 1989: $115-116$.

[VER95] Verhoef BAW, Frederik PM, Bomans PHH, Van der Vusse GJ, Mutsaers PHA, De Lepper ECP, De Voigt MJA. 1995. PIXE microanalysis of trace elements of the normoxic, ischemic and reperfused rat heart. Nucl. Instr. and Meth. B104, 311-316.

[VIS85] Vis RD. 1985. The Proton Microprobe: Applications in the biomedical field, CRC press, Boca Raton (Florida).

[VOR91] Vork MM, Glatz JiC, Surtel DDAM, Knubben HJM, Vusse van der GJ 1991. A sandwich enzyme linked immuno-sorbent assay for the determination of rat heart fatty acid-binding protein using the steptavidin-biotin system. Application to tissue and effuent samples from normoxic rat heart perfusion. Bicchim Biophys Acta 1075, 199-205.

[VOR93] Vork MM, Fatty acid-binding protein in rat heart. 1993. Ph. D. thesis, Maastricht University.

[VUS88] Vusse van der GJ, Van Bilsen M, Willemsen P. Reneman RS. 1988. The myocardial content of fatty acids and phospholipids during the calcium paradox. J Moll Cell Cardiol 20,617-623.

[WAL94] Wallace WE, Rothman JB, Composto RJ, 1994. The stopping power of 1 is 3 MeV hydrogen and helium in polystyrene. Nucl. Instr. and Meth. B84, I-4.

[WAT81] Watt F, Grime GW, Blower GD, Taknos J. 1981. A coupled triple configuration of the Osford microprobe. IEEE Transactions on Nucl. Science, Vol.NS-28, No.2, 1413-1416.

[WAT93] Watt F, Landsberg JP. 1993. Nuclear microseopy: biomedical applications. Nucl. Instr. and Meth. B77, 249-260.

[WAT95] Watt F. 1995. Nuclear microscopy in the life sciences. Nucl. Instr. and Meth. B104, 276-284.

[WE182] Weiss J, Shine KI. 1982. $\mathrm{K}^{+}$accumulation and electrophysiological alterations during early mycocardial ischemia. An Physiol Soc, H318-H3327. 
[WEN91] Wendt-Gallitelli MF, Isenberg 0.1991 . Total and fre myoplasinice calcum during a contraction cycle: $\mathrm{X}$-ray micronalysis in gumea-pig ventricular myocytes. Jof Physiology. $435,349-372$

[WOL84] Wolosewick $\mathbb{J}$. 1984. Cell fine structure and protein antigenicity after polyethylene glucol processing. Science of Biological Specimen Preparation, 83-95.

[ZGL88] Zglinicki von $T$, Uhrik B. 1988. X-ray microanalysis with continuous specimen cooling: is it necessary? J. Microsc, Vol. 151, Pt1, 43 - 47.

[ZIE88] Ziegler JF, Manoyan JM. 1988. The stopping of ions in compounds. Nucl. Instr, and Meth. B35, $215-228$.

[2IE88] Zierold K, Schafer D, 1988. Preparation of cultured and isolated cells for X-ray microanalysis. Scanning Microscopy. Vol.2, no 3, $1775-1790$.

[ZIE80] Zierold K. 1980. Cryofixation of tissue specimens studied by cooling rate measurentents and scanning electron micrascopy. Microscopica acta Vol. 83, number 1, 25-32.

[ZMM6] Zimmerman ANE, Hüismann WC. 1965. Paradoxical influence of calcium ions on the permeability of the cell membranes of the isolated rat heart Narure 6, 646-647.

[ZWIJ93] Zwijnsvoordle van C. 1993. Sofware ontwerp en implementatie voor metingen aan spoorelementen in de scannende protonen microbundel opstelling. Master of Science thesis. VDF/NK 93-03. Eindhoven University of Technology. 


\section{Summary}

Impaired perfusion of the heart readily results in irreversible damage of cardiac muscle cells (cardiac infarct). To date in cardiology the mechanism of ischemic cell death is still incompletely understood. A hypothesis for this mechanism is that cell injury is caused by impaired activity of ion pumps, followed by alterations in the cellular concentrations of ions, such as $\mathrm{Na}^{*}, \mathrm{~K}^{*}, \mathrm{Mg}^{2+}$ and $\mathrm{Ca}^{2+}$. The aim of the present thesis was to explore the possibilities of the Eindhoven proton microprobe set up to study the alterations in elemental concentration of healthy and damaged rat hearts at the cellular level. To this end freeze-dried tissue sections from normoxic, ischemic and reperfused rat hearts were analyzed.

In Chapter 2 the basic theory of the three most commonly used techniques in proton microprobe analysis, i.e., PIXE, RBS and STIM, are presented. The calculation of the elemental concentration and the lowest detectable mass fraction obtainable with a combination of these techniques are also discussed in this chapter. It was shown that even in biological samples with a thickness of several $\mu \mathrm{m}$ for several elements of interest, such as $\mathrm{Na}$ and $\mathrm{Mg}$, thick-target effects have to be taken into account. Calculations indicated that for samples with an areal mass density of $0.6 \mathrm{mg} / \mathrm{cm}^{2}$ neglecting thick target effects would even result in an underestimation of the $\mathrm{Na}$ concentration by a factor of 2 . An experimental validation of these calculations was obtained by proton microprobe analysis of samples with a known and homogeneous concentration distribution of $\mathrm{Na}$ and $\mathrm{K}$. In addition it was shown that the matrix composition of the sample has a minor influence on the elemental concentrations as determined from proton microprobe experiments. As a result in most cases it is sufficient to determine the (local) thickness of the specimen and to use a mean matrix composition for the calculation of the concentration of trace elements present in the sample.

Since the electron microprobe is widely used, playing an important role in analyses in the biomedical field, a practical comparison between the proton and electron microprobe is given in Chapter 3 . To this end the minimum detectable Ca content was determined experimentally using both PIXE and EPMA by analysis of the same set of standards in biologically relevant matrices. It appeared that the use of both sucrose droplets and dialyzed BSA as a matrix material was not satisfactory, due to either non-reproducable results or too high contamination levels, respectively. However, the results obtained with polyethylene glycol (PEG) matrices indicated that the minimum detectable mass fraction for calcium is about two orders of magnitude better for PIXE than for EPMA. In addition it was shown that for Ca contents down to about $10 \mathrm{mg} \cdot \mathrm{kg}^{-1}$ no significant influence of high $\mathrm{K}$ levels on the quantitative detection of $\mathrm{Ca}$ was found.

An extensive description of the adaptations and improvements in experimental procedures and instrumentation of the Eindhoven proton microprobe is provided in Chapter 4 . The most important improvement was the introduction of the STIM technique. Making use of test specimens it was shown that a spatial resolution of about $1 \mu \mathrm{m}$ can be obtained with STIM. STIM analysis of a $3 \mu \mathrm{m}$ thick freeze-dried cryosection of a rat heart showed that the myocardial cells and their boundaries were clearly visible. This result implies that the STIM technique enables one to visualize individual cells. The combination of PIXE/RBS and STM thus makes it possible to 
determine the elemental content of different cells and thus to perform a cell-to-cell comparison within the same tissue section.

In Chapter 5 the preliminary results of proton microprobe analysis of control, normoxic, ischemic and reperfused rat hearts are presented. This study was mainly performed to gain insight into the possible artefacts introduced during the complete process of sample preparation and to verify whether the measured values for the elemental content correspond with values from literature. Isolated, ejecting rat hearts were used to study the influence of ischemia and reperfusion on the content distribution of the heart.

Electron microscope analysis of ultrathin tissue sections showed that a number of artefacts can be easily introduced during the preparative steps. Firstly in several cases in the outer region of the samples serious tissue damage, probably induced during the excision of the biopsies from the ventricular wall, could be observed. Secondly, storage of the biopsies at $-70^{\circ} \mathrm{C}$ for a long period of time as well as the use of warm backing foils to pick up frozen cryosections resulted in altered elemental contents. Finally, utmost care is required to prevent rehydration of the freezedried specimens and consequently possible element migration prior to proton microprobe analysis.

Proton microprobe analysis showed that in ischemic and reperfused tissue sections alterations in $\mathrm{Na}, \mathrm{Mg}, \mathrm{K}$ and $\mathrm{Ca}$ contents can be followed with a spatial resolution in the order of the beam diameter $(-3 \mu \mathrm{m})$. After reperfusion the $\mathrm{K}$ levels decreased as compared to ischemic tissue, whereas an appreciable increase in $\mathrm{Ca}$ content was observed. After reperfusion the $\mathrm{Ca}$ content showed large gradients, which implies that locally large $\mathrm{Ca}$ levels were present. These local variations in $\mathrm{Ca}$ content became visible by mapping of the $\mathrm{Ca}$ content. It is suggested that these locally elevated $\mathrm{Ca}$ levels are caused by the precipitation of calcium phosphate crystals in the mitochondrial compartment of the reperfused myocardial cells.

The maximum alterations in cardiomyocytal ion contents that are to be expected under severely damaging conditions were assessed in Chapter 6 . To this end rat hearts were subjected to the calcium paradox. In addition to the proton microprobe, which was used to determine the alterations in elemental content, light microscopy, STIM and electron microscopy were used to study the histological and morphological changes following the calcium paradox. Light microscope analysis of stained tissue sections revealed severe damage, i.e, the tissue showed no regular structure and cells were not connected anymore and seemed to be torn asunder. Electron microscope analysis showed that the mitochondria contained dense granules. $X$-ray microanalysis of tissue sections using both the proton and electron microprobe showed dramatic alterations in the cellular elemental content in tissue sections from hearts subjected to the calcium paradox. In addition, EPMA analysis showed that the mitochondria contained very high levels of $\mathrm{Ca}$ and $\mathrm{P}$. It is believed that this can be attributed to the massive accumulation of calcium in the mitochondria of the injured cell, since the mitochondria serve as calcium scavengers in case of intracellular Ca overload. It was also found that the calcium paradox created a comparable alteration in elemental content in all analyzed cells, suggesting that all cells were damaged to approximately the same extent. This finding was supported by the observation that $70-90 \%$ of the myocardial muscle cells had lost their $\mathrm{LDH}$ content during the calcium paradox, indicating that the cell membranes of the majority of cardiomyocytes were severely damaged. 
In Chapter 7 a more advanced study of hearts subjected to prolonged ischemia followed by reperfusion is described. Just like the previous results presented in Chapter 5 , the most considerable alterations in mean elemental content of the different cells were observed in tissue sections from reperfused rat hearts. In some cases values for the elemental content were observed that were comparable with values found in hearts subjected to calcium paradox experiments. However, for some elements, such as $\mathrm{P}$ and $\mathrm{S}$, no substantial changes were observed after ischemia and reperfusion. The combined use of PIXE/RBS and the STIM technique revealed that intercellular heterogeneity in elemental content was found, especially in reperfused tissue. For some elements, such as $\mathrm{K}$, this heterogeneity was also found at the end of the ischemic period. This can be explained by the well-known fact that some parts within the myocardial wall are damaged due to ischemia and reperfusion, whereas other parts remain unaffected. Again this result was supported by the finding that only about $5 \%$ of all cells had lost their LDH content.

In conclusion, it was shown in this thesis that the Eindhoven proton microprobe is a valuable tool to study the changes in elemental content due to ischemia and reperfusion at the cellular level. The spatial resolution of about $3 \mu \mathrm{m}$ obtainable during PIXE/RBS experiments does not allow to determine the elemental content of subcellular structures. However ${ }_{n}$ the combined use of PIXE/RBS and the STIM technique makes it possible to determine the mean cellular content of different cells. This enables one to study cellular heterogeneity in healthy and damaged myocardial tissue. 


\section{Samenvatting}

Een verstoorde doorbloeding van het hart (ischemie) leidt snel tot een onherstelbare beschadiging van hartspiercellen (hartinfarct). Tot op heden is niet volledig duidelijk welk mechanisme ten grondslag ligt aan celdood ten gevolge van ischemie. Een hypothese is dat beschadiging van de cel wordt veroorzaakt door een verstoorde aktiviteit van ionenpompen, gevolgd door veranderingen in de cellulaire concentraties van verschillende ionen, zoals $\mathrm{Na}^{+}{ }_{,} \mathrm{K}^{+}, \mathrm{Mg}^{2+}$ en $\mathrm{Ca}^{2+}$. Het doel van het huidige proefschrift was om te onderzoeken welke mogelijkheden de Eindhovense proton microbundel opstelling biedt om op cellulair niveau veranderingen in elementconcentraties in gezond en beschadigd hartspierweefsel te bestuderen. Daartoe zijn met deze opstelling dunne plakjes gevriesdroogd weefsel, afkomstig van normoxische, ischemische en gereperfundeerde rattehartjes, geanalyseerd.

In het algemeen worden tijdens proton microbundel analyses drie verschillende technieken gebruikt, te weten PXE, RBS en STIM. De basistheorie van deze technieken wordt behandeld in Hoofdstuk 2. In dit hoofdstuk worden tevens de berekening van elementconcentraties en de laagst detecteerbare massafraktie, die met een combinatie van de bovenstaande technieken kan worden bepaald, behandeld. Het werd aangetoond dat men zelfs in biologische preparaten met een dikte van enkele $\mu \mathrm{m}$ voor sommige elementen, zoals natrium en magnesium, rekening moet houden met dikke-target effekten. Berekeningen lieten zien dat in preparaten met een massadikte van $0.6 \mathrm{mg} / \mathrm{cm}^{2}$ een tweemaal te lage $\mathrm{Na}$ concentratie wordt gevonden indien men geen rekening houdt met dikke-target effekten. Een experimentele validatie van deze berekeningen werd verkregen door preparaten met een bekende, homogeen verdeelde $\mathrm{Na}$ en $\mathrm{K}$ concentratie te analyseren in de proton microbundelopstelling. Daarnaast werd aangetoond dat de concentraties van de hoofdbestanddelen van het preparaat nauwelijks van invloed zijn op de berekende concentratiewaarden voor de andere elementen die een veel lagere concentratie hebben. Teneinde de concentratie van de spoorelementen te bepalen is het daarom meestal voldoende om de lokale dikte van het preparaat te bepalen en uit te gaan wan een gemiddelde samenstelling van het preparaat.

In Hoofdstuk 3 wordt een vergelijking gemakt tussen de proton microbundel en de elektronenmicroscoop, daar deze latste veelvuldig wordt gebruikt en een belangrijke rol speelt in biomedische analyses. Daartoe werd in dezelfde set ijkpreparaten met zowel PIXE als EPMA de laagst detekteerbare calciumconcentratie bepaald. Deze ijkpreparaten bestonden uit een op biologisch materiaal lijkende matrix, te weten sucrose, gedialyseerd BSA of polyethyleen glycol (PEG), waarin verschillende hoeveelheden $\mathrm{Ca}$ en $\mathrm{K}$ waren opgelost. "Ten gevolge van respectievelijk een slechte reproduceerbaarheid of een te hoog verontreinigungsgehalte voldeden sucrose en BSA niet als matrixmateriaal. De resultaten met PEG als matrixmateriaal toonden echter aan dat de laagst detecteerbare calciumconcentratie voor PLXE twee ordes van grootte lager is dan voor EPMA. Tevens werd aangetoond dat de detektie van lage concentraties calcium (tot ongeveer $10 \mathrm{mg} \mathrm{kg}^{-1}$ ) niet significant wordt beinvloed door de aamwezigheid van grote hoeveetheden $K$.

In Hoofdstuk 4 wordt een uitgebreide beschrijving gegeven van de ampassingeit die zijn 
aangebracht aan de Eindhovense proton microbundelopstelling. De belangrijkste verbetering die is aangebracht is de introduktie van de STIM techniek. Met behulp van testpreparaten werd aangetoond dat met STIM een plaatsresolutie van ongeveer $1 \mu \mathrm{m}$ kan worden bereikt. STIM analyse van $3 \mu \mathrm{m}$ dikke gevriesdroogde cryosecties van een rattehartje toonde aan dat de afzonderlijke bartspiercellen duidelijk zichtbaar waren. De combinatie van PIXE/RBS en STIM maakt het dus mogeljk om de elementconcentratie van verschillende cellen te bepalen en aldus verschillende cellen binnen dezelfde weefselcoupe met elkaar te vergelijken.

De eerste resultaten van proton microbundelanalyse wan controle, normoxische, ischemische en gereperfundeerde rattehartjes worden gepresenteerd in Hoofdstuk 5. Deze studie werd voornamelijk uitgevoerd om inzicht te verkrijgen in de mogelijke artefacten die worden geintroduceerd tijdens de preparatie van het weefsel en om te verifieren of de gemeten concentratiewaarden overeenkomen met literatuurwarden. Teneinde de invloed van ischemie en reperfusie op de concentratieverdeling van verschillende elementen te bepalen werd gebruik gemakt van opstelling warin geisoleerde, werkende rattehartjes aan gecontroleerde omstandigheden, zoals ischemie, kunnen worden blootgesteld.

Analyse van zeer dunne plakjes weefsel met behulp van de elektronenmicroscoop toonde aan dat tijdens de preparatie gemakkelijk een aantal artefacten kunnen worden geïntroduceerd. Ten eerste bleek in een aantal gevallen de buitenste rand van de weefselcoupe ernstig beschadigd te zijn. Deze schade wordt waarschijnlijk toegebracht tijdens de excisie van het biopt uit de hartwand. Tevens bleek dat zowel het langdurig opslaan van weefselbiopten bij een temperatuur van $-70^{\circ} \mathrm{C}$ als het gebruik van ongekoelde dragerfilms om bevroren weefselcoupes op te vangen kan leiden tot veranderde elementconcentraties. Tenslotte moet men uiterste zorg betrachten om te voorkomen dat het gevriesdroogde weefsel weer vocht opneemt, omdat hierdoor de elementen mogelijk reeds woorafgaand aan de proton microbundelanalyse gaan migreren.

Met behulp van de proton microbundel konden veranderingen in $\mathrm{Na}, \mathrm{Mg}, \mathrm{K}$ en $\mathrm{Ca}$ concentraties worden bepaald in ischemisch en gereperfundeerd weefsel met een spatiële resolutie ongeveer ter grootte van de bundeldameter $(\sim-3 \mu \mathrm{mi})$. In vergeijking met ischemisch weetsel zakte de $\mathrm{K}$ concentratie na een periode van reperfusie, terwijl de $\mathrm{Ca}$ concentratie sterk toenam. In gereperfundeerd weefsel liepen de Ca waarden sterk uiteen. Door de Ca concentratie als funktie van de plaats in het weefsel uit te zetten bleek dat er lokaal sterke Ca ophopingen aanwezig waren. Deze lokale ophopingen worden waarschijnlijk veroorzaakt door de neerslag van calciurnfosfaatkristallen in de mitochondrièn.

Hartjes kunnen zeer zwar worden beschadigd door ze bloot te stellen aan de zogenaamde calcium paradox. De resultaten van dit experiment verschaffen daarom inzicht in de maximale veranderingen in elententconcentratie die kunnen optreden als het hart minder zwaar beschadigd wordt, zoals gebeurt tijdens reperfusie na een periode van ischemie. In Hoofdstuk 6 werd met behulp van lichtmicroscopie, STIM en elektronenmicroscopie aangetoond dat de histologie en de morfologie wan het hartweefsel ernstig wordt aangetast ten gevolge van de calcium paradox. Elementanalyse met behulp van zowel de proton microbundel als de elektronentmicroscoop liet tevens zien dat de calcium paradox in alle geanalyseerde cellen een sterk gewijzigde elementconcentratie had veroorzaakt. Dit suggereert dat alle cellen eenzelfde mate van beschadiging hebben opgelopen tijdens de calcium paradox. Deze bevinding werd ondersteund 
door de waarneming dat $70-90 \%$ van de totale LDH inhoud van het hart werd uitgestoten, wat er op duidt dat de celmembranen van de meerderheid van de hartspiercellen ernstig waren beschadigd.

In Hoofdstuk 7 wordt beschreven hoe PIXE/RBS en STIM gecombineerd kunnen worden om de gemiddelde elementconcentratie te bepalen van verschillende cellen in ischemisch en gereperfundeerd hartspierweefsel. De grootste veranderingen in elementconcentratie werden opnieuw waargenomen in weefselcoupes afkomstig uit gereperfundeerde hartjes. In sommige gevallen werden waarden voor de cellulaire concentratie gevonden die vergelijkbaar waren met waarden die werden gevonden in hartjes die waren blootgesteld aan de calcium paradox. In andere gevallen werden echter geen substantiële veranderingen in elementconcentratie waargenomen. Voor sommige elementen werd deze heterogeniteit ook waargenomen aan het eind van de ischemische periode. Dit kan worden verklaard door het feit dat sommige delen van de hartwand beschadigd raken ten gevolge van ischemie en reperfusie, terwijl andere delen niet worden aangetast.

Deze thesis heeft geleid tot de conclusie dat het mogelijk is om met behulp van de Eindhovense proton microbundel op cellulair niveau veranderingen in elementconcentraties te bestuderen in ischemisch en gereperfundeerd hartweefsel. Beperkingen in de spatiële resolutie en de bundelstroom verhinderen de bepaling van elementconcentraties in subcellulaire strukturen. Echter, daar men de gemiddelde cellulaire concentratie van verschillende cellen kan bepalen, is het mogelijk om cellulaire heterogeniteit te bestuderen in gezond en beschadigd hartspierweefsel. 


\section{Publications and abstracts}

\section{Full Papers}

Verhoef BAW, de Goeij JJM, Mutsaers PHA, de Voigt MJA, Bomans PHH, Frederik PM, van der Vusse GJ. 1996. PLXE microanalysis of trace elements of normoxic, ischemic and reperfused rat hearts. Cell. Mol. Biol. 42, 59-67.

Verhoef BAW, Frederik PM, Bomans PHH, van der Vusse GJ, Mutsaers PHA, de Lepper ECP, de Voigt MJA. 1995. PIXE microanalysis of trace elements of the normoxic, ischemic and reperfused rat heart. Nucl. Instr. and Meth. B104, 311-316.

Van Maanen IF, Mutsaers PHA, Verhoef BAW, de Voigt MJA. 1996. Scanning transmission ion microscopy (STIM) with a $3 \mathrm{MeV}$ proton cyclotron beam. Nucl. Instr. and Meth. B113, 382-386.

Micek SL, Verhoef BAW, de Voigt MJA, Bacelar JC, Burger P, Verplancke P, Vermeulen P, Schotanus P. 1992. Development of a special anti-Compton spectrometer. Nucl Instr. and Meth. B64, 282-286

De Voigt MJA, Bacelar JC, Micek SL, Schotanus P, Verhoef BAW, Wintraecken YJE, Vermeulen P. 1995. A novel compact Ge-BGO Compton-suppression spectrometer. Nucl. Instr. and Meth. A356, 362-375.

\section{Abstracts}

Verhoef BAW, Frederik PM, Bomans PHH, van der Vusse GJ, de Voigt MJA. 1995. Proton and electron microscopy as applied to $X$-ray microanalysis of myocardial tissue- a comparison with respect to sensitivity and spatial resolution. NVEM najarsvergadering 1995 , Papendal, The Netherlands

Verhoef BAW, Frederik PM, Bomans PHH, van der Vusse GJ, de Voigt MJA. 1993. Comparison between proton induced and electron induced $X$-ray microanalysis-assessment with microdroplets and preliminary results from cardiac tissue. 12 th Pfefferkorn Conference, Cambridge, UK 


\section{Curriculum Vitae}

De auteur van dit proefschrift werd geboren in Spijk op 23 decenber 1967. Na het behalen van het VWO-B diploma aan de Rjjksscholengemeenschap Wijdschild te Gorinchem in 1985 werd in hetzelfde jaar begonnen met de studie Technische Natuurkunde aan de Technische Universiteit Eindhoven. Zijn afstudeerwerk, met als onderwerp het design van een compacte anti-Compton spectrometer, werd uitgevoerd onder leiding van prof. dr. M.J.A de Voigt in de groep Kernfysische Technieken van de Vakgroep Deeltjesfysica. Na het afronden van deze studie was hij vanaf september 1991 tot maart 1996 werkzaam als Assistent In Opleiding bij de Vakgroep Fysiologie aan de Universiteit Maastricht. De werkzaamheden zoals beschreven in dit proefschrift werden in deze groep en de bovengenoemde groep Kernfysische Technieken uitgevoerd. Vanaf 1 januari 1997 is hij werkzaam als Applicatie Specialist bij de afdeling Surgery van Philips Medical Systems te Best. 


\section{Dankwoord}

Het onderzoek dat is beschreven in dit proefschrift is slechts mogelijk gemaakt door de hulp en steun van velen. Ik wil dan ook iedereen die een bijdrage heef geleverd aan de totstandkoming, van dit proefschrift hartelijk bedanken.

Allereerst wil ik mijn promotores Ger van der Vusse en Martien de Voigt bedanken voor hun niet aflatende inzet om mijn promotie tot een goed einde te brengen. Ger, bedankt voor je goede adviezen en het nauwgezet corrigeren van het manuscript. Helaas lag het tempo waarmee ik nieuwe hoofdstukken inleverde wat lager dan de snelheid waarmee jij ze corrigeerde, daarom vooral ook bedankt voor al je geduld. Martien, tijdens mijp afstudeeronderzoek in joww groep heb je me gemotiveerd om te gaan promoveren. Het doet me daarom goed dat het er, mede door jouw enthousiasme en motivatie tijdens het onderzoek, toch nog van is gekomen.

Ook de twee co-promotores, Peter Frederik en Peter Mutsaers, wil ik bedanken voor al hun goede ideeën tijdens het onderzoek en kritische beoordeling van het proefschrift. Peter $F$, bedankt woor de warme ontvangst in de EM-groep en de leuke en leerzame tijd die ik met je heb mogen doorbrengen. $\mathrm{Ik}$ denk dat onze samenwerking heeft bewezen dat de elektronenmicroscoop en de proton microbundel elkaar perfekt aanvullen. Peter M., tijdens het eerste jaar van mijn onderzoek is de nieuwe microbundelopstelling, jouw 'kindje', in gebruik genomen. Ik heb vaak dankbaar gebruik gemaakt van je enorme kennis op het gebied van microbundels en PIXE. Bedankt voor al je hulp bij de experimenten en je adviezen tijdens het schrijven van het proefschrift.

Jeroen dle Goeij wil ik speciaal bedanken voor zijn bijdrage aan het schrijven van het proefschrift en het kritisch leren beoordelen van mijn werk. Ik heb altijd veel plezier beleefd aan onze pittige discussies en besef nu hoe belangrijk "een goede kleermaker" is.

Alle preparaten die ik heb gebruikt tijdens het onderzoek zijn door de handen van Paul Bomans gegaan. Paul, ik wil je bedanken voor het prepareren en snijden van alle samples, je assistentie bij de metingen met de CM-12 en alle andere dingen die je voor me hebt gedaan. Ik zou je dan ook van harte een beter nationaal voetbalelftal gunnen, maar ja.

De perfusie-experimenten zijn uitgevoerd door Richard Cornellussen en Michael Vork. Richard en Michaël, bedankt voor jullie hulp. Michaël, ik heb het altijd erg leuk gevonden dat jij mijn kamergenoot was op de dagen dat ik in Maastricht was. Mijn eerste kennismaking met het Maastrichtse uitgaansleven onder jouw leiding was onvergetelijk.

Guido Zonneveld en ikzelf waren de eerste gebruikers van de nieuwe microbundelopstelling. Guido, bedankt voor de leuke tijd en de gezamenlijke metingen tot in de late uurtjes. Ik ben er nog steeds trots op dat je nog nooit een foutje in al mijn computerprogramma's hebt kunnen vinden. Voor het uitvoeren van experimenten met de microbundel is het essentieel te kunnen vertrouwen op een goed werkend cyclotron. Ik wil daarom alle leden van de cyclotron bedrijfsgroep bedariken voor hun bijdrage. Met name Jan van den Berg en Frits van Hirtum waren altijd bereid te helpen als er problemen waren (vaak op zaterdag of 's nachts).

Tijdens experimenten met de microbundel is veelvuldig gebruik gemaakt van de computer. Ik stel het daarom zeer op prijs dat ik altijd kon rekenen op de hulp van Wim Verseijden als er problemen waren op computergebied. Wim, bedankt voor je geduld. Als Wim er niet was om mij te helpen 
met computerproblemen, kon ik gelukkig nog terugvallen op David Simons. David, bedankt voor je hulp, met name ook bij het fabriceren van de figuren.

De bijdrage van afstudeerders aan het onderzoek aan hartweefsel met behulp van de microbundel is zeer groot geweest. De volgende afstudeerders wil ik daarom hartelijk bedanken voor al hun hulp tijdens de lange meetdagen: Hans Jenniskens, Erik de Lepper, Marco van Uden, Katuschka van Nuland, Roger Jacobs en Peter Brands. Naast alle afstudeerders hebben ook stagiaires een belangrijke bijdrage geleverd aan het onderzoek aan het hartspierweefsell. Hierbij ook alle stagiaires bedankt voor al het werk.

Ondanks het feit dat ik nu steeds vaker met plezier terugkijk op mijn promotietijd, zijn er periodes geweest waarop ik wat minder vertrouwen had in de goede afloop. Vooral op die momenten is het enorm belangrijk geweest dat er vrienden waren die meer vertrouwen in mij hadden dan ikzelf. Met name Ralph Soeren en Albert Mangnus ben ik daarom zeer erkentelijk voor alle steun. Ralph en Albert, bedankt voor jullie vriendschap.

Tenslotte: mijn allerliefste Ageeth, bedankt voor het feit dat je er altijd voor me was. Jouw begrip, geduld en support waren onmisbaar. 